\title{
Aerodynamic Response of Turbomachinery Blade Rows to Convecting Density Wakes
}

\author{
by

\section{Hettithanthrige Sanith Wijesinghe} \\ MEng. Aeronautical Engineering, Imperial College of Science, Technology and Medicine
}

\section{Submitted to the Department of Aeronautics and Astronautics}

in partial fulfillment of the requirements for the

degree of

\section{Master of Science}

at the

\section{Massachusetts Institute of Technology}

September 1998

(C) 1998 Massachusetts Institute of Technology.

All rights reserved.

Author

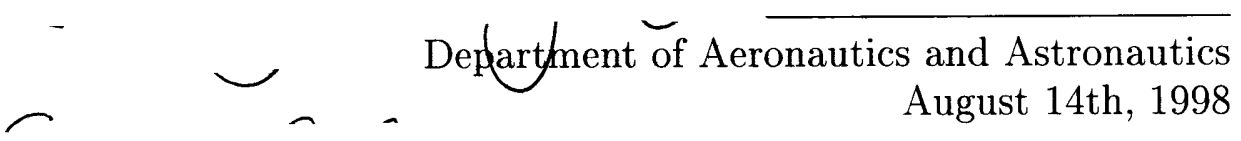

Certified by

Professor Eugene E. Covert Professor of Aeronautics and Astronautics, Emeritus

Certified by_

Thesis Supervisor

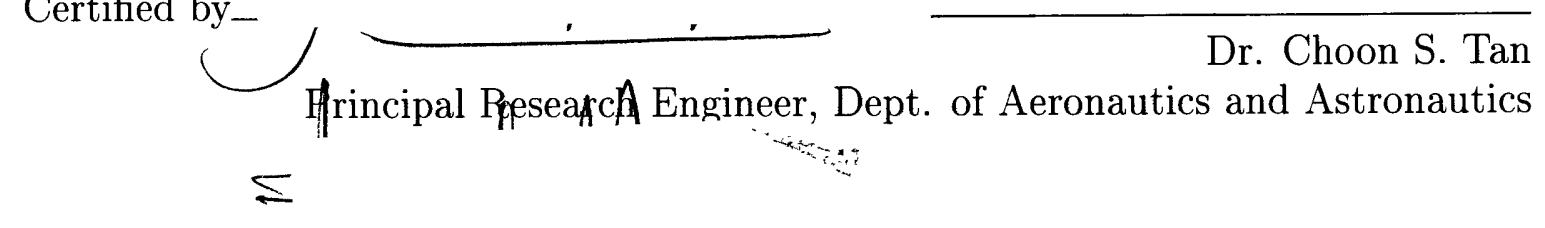

Accepted by

Professor Jaime Peraire

Associate Professor of Aeronautics and Astronautics

MASSACHUSETTS INSTITUTE

Chairman, Department Graduate Committee

OF TECHNOLOGY 


\title{
Aerodynamic Response of Turbomachinery Blade Rows to Convecting Density Wakes
}

\author{
by \\ Hettithanthrige Sanith Wijesinghe \\ Submitted to the Department of Aeronautics and Astronautics on August 14th, 1998 \\ in partial fulfillment of the requirements for the degree of \\ Master of Science
}

\begin{abstract}
Density wakes have been recently identified as a possible new source for high cycle fatigue failure in the compressor blades of modern turbomachinery. In order to characterize the density wake induced force and moment fluctuations in compressor blades a two-dimensional computational study has been conducted in viscous compressible flows with Mach numbers ranging from $M_{\infty}=0.15$ to $M_{\infty}=0.87$ and flow Reynolds number $\operatorname{Re}\left(c, U_{\infty}\right) \approx 700,000$.

Parametric tests were conducted at each flow Mach number to establish trends for the change in the maximum fluctuation of the blade force and moment coefficients with the changes in the density wake width $0.1 \leq w / c \leq 1.0$ and the density ratio $0.25 \leq \rho_{2} / \rho_{1} \leq 2.0$. Results indicate the magnitude of the blade force and moment fluctuations to scale with (1) the non-dimensional density wake width $w / c,(2)$ a non-dimensional density parameter $\rho^{*}$ and (3) flow Mach number $M_{\infty}$.

The viscous flow simulations have also indicated (1) periodic vortex shedding at the blade trailing edge and (2) separation bubbles on the blade suction surface which generate additional force and moment fluctuations with amplitudes $\pm(10-100 \%)$ about the time averaged mean values. These flow features represent possible additional sources for high cycle fatigue failure.

Simple functional relationships have also been derived at each flow Mach number to quantify the force and moment fluctuations described above. In addition a simple cascade flow model has been developed in conjunction with the computational study to help determine the trends in the force and moment fluctuations with varying density wake properties and compressor geometries.
\end{abstract}

Thesis Supervisor: Prof. Eugene E. Covert

Title: Professor of Aeronautics and Astronautics, Emeritus

Thesis Supervisor: Dr. Choon S. Tan

Title: Principal Research Engineer, Dept. of Aeronautics and Astronautics 


\section{ACKNOWLEDGMENTS}

I would like to take this opportunity to acknowledge the love and support of my parents throughout the years. They have always allowed me to make my own decisions in life and have supported me through those decisions. I dedicate this thesis to them for helping me achieve all my goals.

I wish to thank Prof. Covert for providing me the opportunity to work on this project. His confidence and patience in me throughout these past 2 years is greatly appreciated. The analysis presented here is due in whole to the many discussion we have had. His excellent advising and mentoring throughout the project has made this a most pleasurable experience.

I would like to thank Choon for his thoughtful advice and comments and for helping me focus on the important research issues. I would also like to thank him for carefully proof reading this document many many times over. His suggestions have greatly improved the final version.

I would like to thank Prof. Frank Marble for his helpful discussions and comments. His original work provided the motivation for this research. I am also grateful to Becky Ramer for her initial work on this project.

A big thank you to Brian, Ken, Asif and Yang for their contribution to a lively office atmosphere and for always being available to discuss wild and crazy ideas! Thanks also to everyone at the GSC and the SLSA who successfully managed to divert my attention away from research and made sure I invested all my free time to help organize random events. It has been a most worthwhile experience!

This work has been supported by the Air Force Office of Scientific Research and supervised by Major Brian Sanders, Program Manager, under contract number F49620-941-0202. This support is gratefully acknowledged. 


\section{CONTENTS}

$\begin{array}{ll}\text { Abstract } & \mathbf{2}\end{array}$

$\begin{array}{ll}\text { Acknowledgments } & 3\end{array}$

$\begin{array}{lr}\text { List of Figures } & 8\end{array}$

List of Tables $\quad 20$

$\begin{array}{ll}\text { Nomenclature } & 23\end{array}$

1 Introduction $\quad 27$

1.1 Background . . . . . . . . . . . . . . . . . 27

1.2 Physical Origin of Unsteadiness . . . . . . . . . . . . . . . . . . . 29

1.3 Theoretical Background: Marble's Linearized Analysis . . . . . . . . . . . . 30

1.4 Inviscid Flow Simulations . . . . . . . . . . . . . . . . . . 33

1.4.1 Conclusions Based on Inviscid Results . . . . . . . . . . . . . 37

1.5 Questions Posed by the Current Research . . . . . . . . . . . . . . 37

1.6 Technical Approach . . . . . . . . . . . . . . . . . . . . . 38

1.7 Thesis Contributions . . . . . . . . . . . . . . . . . . . 40

1.8 Thesis Organization $\ldots \ldots \ldots \ldots \ldots$. . . . . . . . . . . . . . . . .

2 Viscous Flow Solver $\quad 43$

2.1 Features of The Viscous Flow Solver . . . . . . . . . . . . . . . . . 43

2.2 Computational Grid . . . . . . . . . . . . . . . 45

2.3 Cascade Geometry and Blade Profile . . . . . . . . . . . . . 47

2.4 Density Wake Profile . . . . . . . . . . . . . . . . . . 47

2.5 Converged Solutions . . . . . . . . . . . . . . . . 49 
2.6 Non-Dimensionalization . . . . . . . . . . . . . . . 50

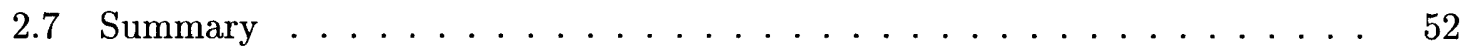

3 Viscous Results: Baseline Solutions $\quad 53$

3.1 Baseline Force and Moment Response . . . . . . . . . . . . . . . . 53

3.1 .1 Run $1: \mathbf{M}_{\infty}=0.15 \ldots \ldots \ldots \ldots \ldots$

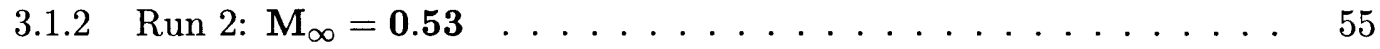

3.1 .3 Run $3: \mathbf{M}_{\infty}=0.63 \ldots \ldots \ldots \ldots$

3.1 .4 Run $4: \mathbf{M}_{\infty}=\mathbf{0 . 8 7} \ldots \ldots \ldots \ldots \ldots$

3.2 Vortex Shedding Frequency and Strouhal Number . . . . . . . . 68

3.3 Summary . . . . . . . . . . . . . . . . . . 70

4 Viscous Results: Response to Density Wakes $\quad 73$

4.1 Force and Moment Profiles: Initial characterization . . . . . . . . . . . . . . 74

4.2 Primary Response . . . . . . . . . . . . . . . . . 76

4.2 .1 General Flow Features . . . . . . . . . . . . . . . 76

4.2 .2 Run $4: \mathbf{M}_{\infty}=\mathbf{0 . 8 7} \ldots \ldots \ldots \ldots \ldots$

4.2 .3 Parametric Study . . . . . . . . . . . . . . . . . . . 84

4.3 Secondary Response . . . . . . . . . . . . . . . . . . . 93

4.3 .1 General Flow Features . . . . . . . . . . . . . . . . . 93

$4.3 .2 \quad$ Parametric Study . . . . . . . . . . . . . . . . . . 96

4.3 .3 Summary . . . . . . . . . . . . . . . . . 101

5 Cascade Flow Model $\quad 107$

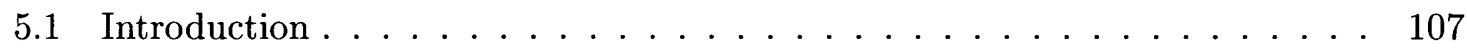

5.2 Modeling Assumptions . . . . . . . . . . . . . . . . . . . . . 108

5.3 Induced velocities . . . . . . . . . . . . . . . 110

5.3.1 Circulation Strength of The Counterrotating Vortices _. . . . . 111

5.4 Solution Procedure . . . . . . . . . . . . . . . . . . . . . 113

5.5 Steady State Model Validation . . . . . . . . . . . . . . 115

5.6 Quasi-Steady Model Validation . . . . . . . . . . . . . . . . . 118

5.7 Parametric Results . . . . . . . . . . . . . . . . . . 121

5.7.1 Lift and Moment Sensitivity to Density Wake Properties . . . . . . . 121 
5.7.2 Lift and Moment Sensitivity to Cascade Geometry . . . . . . . . 121

5.8 Summary . . . . . . . . . . . . . . . . . . 122

6 Conclusions And Further Work $\quad 125$

6.1 Summary . . . . . . . . . . . . . . . . . 125

6.2 Conclusions Based On The Viscous Results _ . . . . . . . . . . . . 126

6.3 Conclusion Based On The Cascade Model Results . . . . . . . . . . . . 126

6.4 Suggestions For Further Work . . . . . . . . . . . . . . . 127

$\begin{array}{lr}\text { Bibliography } & 129\end{array}$

A Baseline Flow Results $\quad 133$

B Viscous Flow Force And Moment Fluctuation Profiles 141

C Compressibility Scaling Of The Maximum Force and Moment Fluctua$\begin{array}{ll}\text { tions } & 169\end{array}$

$\begin{array}{ll}\text { D Cascade Model : Matlab Source Code } & 177\end{array}$ 


\section{List OF FiguRES}

1-1 Density wake convecting through a compressor blade row. . . . . . . . .

1-2 Lift coefficient fluctuation during passage of a density discontinuity over a flat plate. $\lambda$ is the position of the density discontinuity as it convects along the flat plate. The flat plate lies between $|\lambda| \leq 1 \ldots \ldots \ldots$

1-3 Moment coefficient fluctuation during passage of a density discontinuity over a flat plate. $\lambda$ is the position of density discontinuity as it convects along the flat plate. The flat plate lies between $|\lambda| \leq 1 . \ldots \ldots \ldots$

1-4 Perturbation velocity vectors during passage of a density wake of width $0.2 c$ and density ratio 0.5 through the NACA4F blade row. The flow is inviscid and incompressible. $\tau=0.65 \ldots \ldots \ldots \ldots \ldots$

1-5 Fluctuation in (a) azimuthal force coefficient and (b) moment coefficient (positive counter-clockwise about the mid-chord) during passage of density wakes. $\Delta C y=C y_{\max }-C y_{\text {mean }}, \Delta C m=C m_{\max }-C m_{\text {mean }}$ where $C y_{\text {mean }}=0.75, C m_{\text {mean }}=-0.13$. Reproduced from Ramer. . . . . . .

1-6 Maximum fluctuation in the azimuthal force coefficient (from steady state) during passage of density wakes through the NACA4F blade row. $\Delta C y=$ $\left(C y_{\max }-C y_{\text {mean }}\right) / C y_{\text {mean }}$. Reproduced from Ramer. . . . . . . . . 36

1-7 Maximum fluctuation in moment coefficient (from steady state) during passage of density wakes through the NACA4F blade row. $\Delta C y=\left(C y_{\max }-\right.$ $\left.C y_{\text {mean }}\right) / C y_{\text {mean }}$. Reproduced from Ramer. . . . . . . . . . . .

2-1 Schematic of the computational domain and boundary conditions. . . . . 45

$2-2 \quad \mathrm{O}$-grid detail at the blade leading edge. $\ldots \ldots \ldots \ldots$

$2-3 \quad \mathrm{O}$-grid detail at the blade trailing edge. . . . . . . . . . . 47 
2-4 50\% span section of the LSRC Stator-B blade used for the viscous simulations. $\mathrm{X}$ denotes the location about which moments are evaluated $(0.42 \mathrm{c}$,

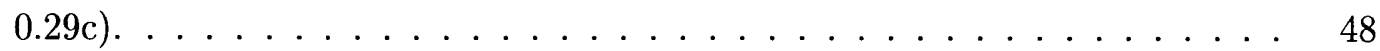

2-5 Density wake profile. $w / c=0.2, \rho_{2} / \rho_{1}=0.5 \ldots \ldots \ldots \ldots$

2-6 Convergence history for the L2 norm of density $\rho \times$ energy e. $M_{\infty}=0.53$. $\quad 50$

3-1 Vorticity contours indicating vortex shedding behind the blade trailing edge. $M_{\infty}=0.15 . \operatorname{Re}\left(c, U_{\infty}\right)=620,000$. The blade trailing edge is located at $\approx 0.40 \times$ (spacing of same-sign vortices) to the right of the left boundary. .

3-2 Trajectory of consecutive shear layer wave peaks at an arbitrary time instance. $M_{\infty}=0.15 \ldots \ldots \ldots \ldots \ldots \ldots \ldots$

3-3 Discrete fourier transform of the baseline force and moment coefficients. $d f t(X)$ is the discrete fourier transform of the time signal $X . \mu=$ nondimensional frequency. $M_{\infty}=0.15 \ldots \ldots \ldots \ldots$

3-4 Vorticity contours indicating vortex shedding behind the blade trailing edge. $M_{\infty}=0.53$. $\operatorname{Re}\left(c, U_{\infty}\right)=630,000$. The blade trailing edge is located at $\approx 0.35 \times$ (spacing of same-sign vortices) to the right of the left boundary. .

3-5 Trajectory of consecutive same-sign vortices at an arbitrary time instance.

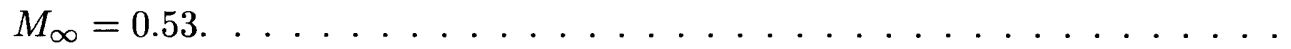

3-6 Discrete fourier transform of the baseline force and moment coefficients. $\operatorname{dft}(X)$ is the discrete fourier transform of the time signal $X . \mu=$ nondimensional frequency. $M_{\infty}=0.53 \ldots \ldots \ldots \ldots \ldots$

3-7 Vorticity contours indicating vortex shedding behind the blade trailing edge. $M_{\infty}=0.63 . \operatorname{Re}\left(c, U_{\infty}\right)=580,000$. The blade trailing edge is located at $\approx 0.50 \times$ (spacing of same-sign vortices) to the right of the left boundary. .

3-8 Trajectory of consecutive same-sign vortices at an arbitrary time instance.

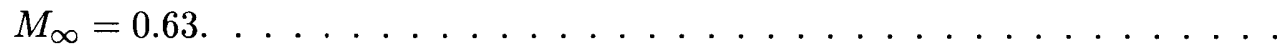

3-9 Discrete fourier transform of the baseline force and moment coefficients. $\operatorname{dft}(X)$ is the discrete fourier transform of the time signal $X . \mu=$ nondimensional frequency. $M_{\infty}=0.63 \ldots \ldots \ldots \ldots 61$

3-10 Mach number contours indicating extent of supersonic region in Run 4. Contours range from $M=1.0$ to $M=1.5$ in steps of $0.05 . M_{\infty}=0.87 \ldots \ldots$ 
3-11 Vorticity contour images indicating vortex shedding behind the blade trailing edge. $M_{\infty}=0.87$. $R e\left(c, U_{\infty}\right)=800,000$. The blade trailing edge is located at $\approx 0.50 \times$ (spacing of same-sign vortices) to the right of the left boundary.

3-12 Trajectory of consecutive same sign vortices at an arbitrary time instance. $M_{\infty}=0.87 \ldots \ldots \ldots \ldots \ldots \ldots \ldots \ldots \ldots \ldots \ldots \ldots \ldots \ldots \ldots \ldots \ldots \ldots \ldots$

3-13 Discrete fourier transform of the baseline force and moment coefficients. $\operatorname{dft}(X)$ is the discrete fourier transform of the time signal X. $\mu=$ nondimensional frequency. $M_{\infty}=0.87 \ldots \ldots \ldots \ldots \ldots$

3-14 Pressure contours of the baseline solution for Run 4. The pressure waves can be seen as dark lines extending across the blade passage. $M_{\infty}=0.87$. $\operatorname{Re}\left(c, U_{\infty}\right)=0.8 \times 10^{6}$

3-15 Schlieren image of upstream traveling pressure waves behind a flat plate cascade from Lawaczeck. The Flow is from left to right. $M$ (trailing edge) $\approx 0.80 . \operatorname{Re}\left(c, U_{\infty}\right)=0.8 \times 10^{6} \ldots \ldots \ldots \ldots$

3-16 Location of upstream traveling pressure waves at specific time instances. $\tau=0.0$ is an arbitrary time instance. $M_{\infty}=0.87 . \ldots \ldots \ldots$

3-17 Fluctuation in the shock wave position $x_{s}$ and the corresponding frequency spectrum. Mean shock location $=0.25 \times \max ($ surf.distance). $\operatorname{dft}(X)$ is the discrete fourier transform of time signal $X \ldots \ldots \ldots \ldots$

3-18 Fluctuation in the static pressure rise across the shock wave $\Delta C p_{s}$ caused by the upstream traveling pressure waves. Mean $\Delta C p_{s}=0.54 . \operatorname{dft}(X)$ is the discrete fourier transform of the time signal $X \ldots \ldots \ldots$

4-1 Fluctuations in (1) azimuthal force coefficient, (2) axial force coefficient and (3) moment coefficient (positive clockwise about the mid chord) during passage of a density wake of width $0.2 c$ and density ratio $\rho_{2} / \rho_{1}=0.5$. 3 distinct regions can be identified in the response. $M_{\infty}=0.15 \ldots \ldots \ldots$

4-2 Density contour image showing passage of density wake of width $0.2 c$ and density ratio $0.5 . M_{\infty}=0.15 . \tau=0.04 \ldots \ldots \ldots \ldots$

4-3 Density contour image showing passage of density wake of width $0.2 c$ and density ratio $0.5 . M_{\infty}=0.15 . \tau=0.53 . \ldots \ldots \ldots$ 
4-4 Density contour image showing passage of density wake of width $0.2 c$ and density ratio $0.5 . M_{\infty}=0.15 . \tau=0.78 \ldots \ldots \ldots \ldots$

4-5 Density contour image showing passage of density wake of width $0.2 c$ and density ratio $0.5 . M_{\infty}=0.15 . \tau=1.28 \ldots \ldots \ldots \ldots \ldots$

4-6 Suction surface pressure distribution during passage of a density wake width $0.2 c$ and density ratio $0.5 . M_{\infty}=0.15 \ldots \ldots \ldots$

4-7 Change in blade pressure coefficient during passage of a density wake width $0.2 c$ and density ratio $0.5 . \tau=0.32, M_{\infty}=0.15 \ldots \ldots \ldots$

4-8 The change in (1) static pressure difference across the shock wave, (2) azimuthal force coefficient and (3) blade shock location during passage of a density wake width $0.1 c$ and density ratio $0.25 . M_{\infty}=0.87 \ldots \ldots$

4-9 Blade suction surface pressure contours showing the temporary suppression of the blade passage shock wave during passage of a density wake of width $0.4 c$ and density ratio 0.25 . The dark band at $x / c=0.25$ is the shock front.

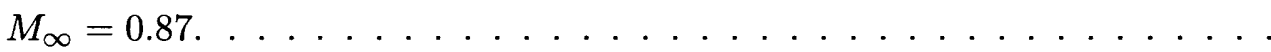

4-10 Changes in the maximum deflection of the shock wave as a function of the density wake width $w / c$ and density parameter $\rho^{*} \ldots \ldots \ldots 84$

4-11 Maximum fluctuation in the force and moment coefficients as functions of density wake width and density parameter $\rho^{*}$. The straight lines joining data points are to help aid clarity. $M_{\infty}=0.15 \ldots \ldots \ldots \ldots$

4-12 Maximum fluctuation in the force and moment coefficients as functions of density wake width and density parameter $\rho^{*}$. The straight lines joining data points are to help aid clarity. $M_{\infty}=0.53 . \ldots \ldots \ldots$

4-13 Maximum fluctuation in the force and moment coefficients as functions of density wake width and density parameter $\rho^{*}$. The straight lines joining data points are to help aid clarity. $M_{\infty}=0.63 . \ldots \ldots \ldots$

4-14 Maximum fluctuation in the force and moment coefficients as functions of density wake width and density parameter $\rho^{*}$. The straight lines joining data points are to help aid clarity. $M_{\infty}=0.87 . \ldots \ldots \ldots$

4-15 Mach number contour image showing boundary layer deformation during passage of a density wake of width $0.2 c$ and density ratio $0.5 . \quad M_{\infty}=0.15$.

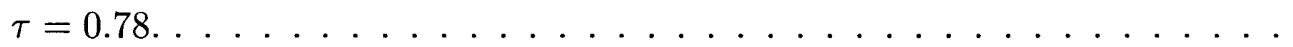


4-16 Mach number contour image showing boundary layer deformation during passage of a density wake of width $0.2 c$ and density ratio $0.5 . \quad M_{\infty}=0.15$.

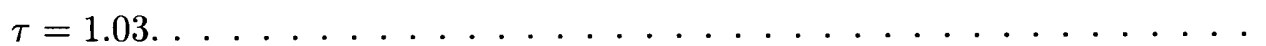

4-17 Mach number contour image showing boundary layer deformation during passage of a density wake of width $0.2 c$ and density ratio $0.5 . M_{\infty}=0.15$.

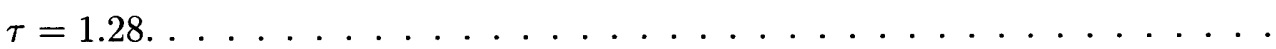

4-18 Mach number contour image showing boundary layer deformation during passage of a density wake of width $0.2 c$ and density ratio $0.5 . \quad M_{\infty}=0.15$.

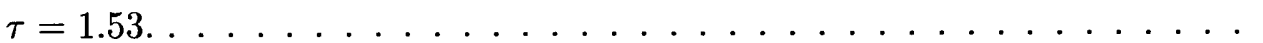

4-19 The fluctuation of the flow separation and re-attachment points of the suction surface boundary layer during passage of a density wake of width $0.2 c$ and density ratio $0.5 . M_{\infty}=0.15 \ldots \ldots \ldots \ldots \ldots$

4-20 Vorticity contour image showing the disruption of regular vortex shedding behind the blade trailing edge. The density wake is located $1.0 c$ downstream of the trailing edge entrained inside the vortex wake. $\tau=3.1, w / c=0.2$, $\rho_{2} / \rho_{1}=0.5, M_{\infty}=0.87 \ldots \ldots \ldots \ldots \ldots \ldots \ldots \ldots \ldots \ldots \ldots$

4-21 Discrete fourier transform of the secondary response force and moment coefficient fluctuations. $w / c=0.2, \rho_{2} / \rho_{1}=0.5, M_{\infty}=0.87 . \operatorname{dft}(X)$ is the discrete fourier transform of the time signal $X . \mu=$ non-dimensional frequency. .

4-22 The maximum change in the suction surface separation point from the mean baseline position as a function of wake width and density ratio. $M_{\infty}=0.15$

4-23 The maximum change in the suction surface separation point from the mean baseline position as a function of wake width and density ratio. $M_{\infty}=0.53 . \quad 100$

4-24 The maximum change in the suction surface separation point from the mean baseline position as a function of wake width and density ratio. $M_{\infty}=0.63$.

4-25 The maximum change in the suction surface separation point from the mean baseline position as a function of wake width and density ratio. $M_{\infty}=0.87$.

4-26 The maximum fluctuation in the blade azimuthal force, axial force and moment coefficients in the secondary response region for varying density wake widths and density ratios. $M_{\infty}=0.15 \ldots \ldots \ldots \ldots$ 
4-27 The maximum fluctuation in the blade azimuthal force, axial force and moment coefficients in the secondary response region for varying density wake widths and density ratios. $M_{\infty}=0.53 \ldots \ldots \ldots \ldots$

4-28 The maximum fluctuation in the blade azimuthal force, axial force and moment coefficients in the secondary response region for varying density wake widths and density ratios. $M_{\infty}=0.63 \ldots \ldots \ldots \ldots$

4-29 The maximum fluctuation in the blade azimuthal force, axial force and moment coefficients in the secondary response region for varying density wake widths and density ratios. $M_{\infty}=0.87 \ldots \ldots \ldots \ldots$. . . . . . . 104

5-1 Model schematic indicating flat plate cascade and counterrotating vortex pairs. 109

5-2 Change in circulation strength of the convecting vortices. $\mathbf{v}=s_{1} \xi+s_{2}$ where $\xi$ is the location of the convecting vortex and $s_{1}$ and $s_{2}$ are constants which ensure the tanh function is evaluated between -3 and $+3 \ldots \ldots \ldots$

5-3 Cascade model results for the change in cascade interference coefficient $C l_{\text {cascade }} / C l_{\text {airfoil }}$ with space-chord ratio and stagger angle. No. of panels $=1 . \ldots \ldots$

5-4 Weinig's conformal mapping prediction for the cascade interference coefficient $k_{o}\left(C l_{\text {cascade }} / C l_{\text {airfoil }}\right)$ as a function of stagger angle $\gamma_{e f f}$ and space-chord

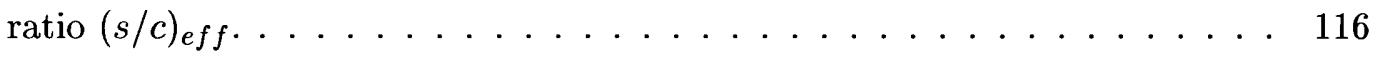

5-5 Cascade model results for the change in cascade interference coefficient $C l_{\text {cascade }} / C l_{\text {airfoil }}$ with space-chord ratio and stagger angle. No. of panels $=5 . \ldots \ldots 117$

5-6 Cascade model results for the change in cascade interference coefficient $C m_{\text {cascade }} / \mathrm{Cm}_{\text {airfoil }}$ with space-chord ratio and stagger angle. No. of panels $=5 . \ldots \ldots 117$

5-7 Comparison of the quasi-steady model results and the inviscid CFD results for the fluctuation in the blade force and moment coefficients during passage of a density wake of width $0.1 c$ and density ratio $0.5 . \quad \Delta C l=\left(C l_{\max }-\right.$ $\left.C l_{\text {mean }}\right) /\left(C l_{\text {mean }}\right), \Delta C m=\left(C m_{\text {max }}-C m_{\text {mean }}\right) /\left(C m_{\text {mean }}\right) \ldots \ldots$

5-8 Cascade lift and moment fluctuation sensitivity to density wake width $w / c$ and density parameter $\rho^{*}$ (measure of density ratio). The NACA4F cascade geometry is used for all tests. Solid lines indicate the model results. Dashed lines indicate the inviscid CFD results. . . . . . . . . . . . . . 122 
5-9 Cascade lift and moment sensitivity to cascade stagger angle and space-chord ratio. Density wake of width $0.1 c$ and density ratio 0.5 is used for all tests.

A-1 Time averaged blade pressure distribution. $M_{\infty}=0.15 \ldots \ldots \ldots \ldots$

A-2 Time averaged blade pressure distribution. $M_{\infty}=0.53 . \ldots \ldots \ldots \ldots$

A-3 Time averaged blade pressure distribution. $M_{\infty}=0.63 . \ldots \ldots \ldots$

A-4 Time averaged blade pressure distribution. $M_{\infty}=0.87 \ldots \ldots \ldots \ldots$

A-5 The force and moment coefficient fluctuation in the baseline solution of Run 1. $C y, C x$ and $C m$ are the blade azimuthal force, axial force and moment coefficients respectively. $\tau=$ convective time scale. $M_{\infty}=0.15 \ldots \ldots 136$

A-6 The force and moment coefficient fluctuation in the baseline solution of Run 2. $C y, C x$ and $C m$ are the blade azimuthal force, axial force and moment coefficients respectively. $\tau=$ convective time scale. $M_{\infty}=0.53 \ldots \ldots 136$

A-7 The force and moment coefficient fluctuation in the baseline solution of Run 3. $C y, C x$ and $C m$ are the blade azimuthal force, axial force and moment coefficients respectively. $\tau=$ convective time scale. $M_{\infty}=0.63 \ldots \ldots 137$

A-8 The force and moment coefficient fluctuation in the baseline solution of Run 4. $C y, C x$ and $C m$ are the blade azimuthal force, axial force and moment coefficients respectively. $\tau=$ convective time scale. $M_{\infty}=0.87 \ldots \ldots \ldots 137$

A-9 Time averaged boundary layer properties. $M_{\infty}=0.15 \ldots \ldots \ldots \ldots$

A-10 Time averaged boundary layer properties. $M_{\infty}=0.53 \ldots \ldots \ldots \ldots$

A-11 Time averaged boundary layer properties. $M_{\infty}=0.63 \ldots \ldots \ldots \ldots$

A-12 Time averaged boundary layer properties. $M_{\infty}=0.87 \ldots \ldots \ldots \ldots$

B-1 Fluctuation in blade force and moment coefficients. $w / c=0.1, \rho_{2} / \rho_{1}=0.25$, $M_{\infty}=0.15 \ldots \ldots \ldots \ldots \ldots \ldots \ldots \ldots \ldots \ldots \ldots \ldots \ldots \ldots \ldots \ldots \ldots \ldots \ldots$

B-2 Fluctuation in blade force and moment coefficients. $w / c=0.1, \rho_{2} / \rho_{1}=0.50$, $M_{\infty}=0.15 \ldots \ldots \ldots \ldots \ldots \ldots \ldots \ldots \ldots \ldots \ldots \ldots \ldots \ldots \ldots \ldots \ldots$

B-3 Fluctuation in blade force and moment coefficients. $w / c=0.1, \rho_{2} / \rho_{1}=0.75$, $M_{\infty}=0.15 \ldots \ldots \ldots \ldots \ldots \ldots \ldots \ldots \ldots \ldots \ldots \ldots \ldots \ldots \ldots \ldots \ldots \ldots \ldots$

B-4 Fluctuation in blade force and moment coefficients. $w / c=0.1, \rho_{2} / \rho_{1}=2.00$, $M_{\infty}=0.15 \ldots \ldots \ldots \ldots \ldots \ldots \ldots \ldots \ldots \ldots \ldots \ldots \ldots \ldots \ldots \ldots \ldots \ldots \ldots \ldots$ 
B-5 Fluctuation in blade force and moment coefficients. $w / c=0.1, \rho_{2} / \rho_{1}=0.25$, $M_{\infty}=0.53$.

B-6 Fluctuation in blade force and moment coefficients. $w / c=0.1, \rho_{2} / \rho_{1}=0.50$, $M_{\infty}=0.53$.

B-7 Fluctuation in blade force and moment coefficients. $w / c=0.1, \rho_{2} / \rho_{1}=0.75$, $M_{\infty}=0.53$.

B-8 Fluctuation in blade force and moment coefficients. $w / c=0.1, \rho_{2} / \rho_{1}=2.00$, $M_{\infty}=0.53$.

B-9 Fluctuation in blade force and moment coefficients. $w / c=0.1, \rho_{2} / \rho_{1}=0.25$, $M_{\infty}=0.63$.

B-10 Fluctuation in blade force and moment coefficients. $w / c=0.1, \rho_{2} / \rho_{1}=0.50$, $M_{\infty}=0.63$.

B-11 Fluctuation in blade force and moment coefficients. $w / c=0.1, \rho_{2} / \rho_{1}=0.75$, $M_{\infty}=0.63$.

B-12 Fluctuation in blade force and moment coefficients. $w / c=0.1, \rho_{2} / \rho_{1}=2.00$, $M_{\infty}=0.63$.

B-13 Fluctuation in blade force and moment coefficients. $w / c=0.1, \rho_{2} / \rho_{1}=0.25$, $M_{\infty}=0.87$.

B-14 Fluctuation in blade force and moment coefficients. $w / c=0.1, \rho_{2} / \rho_{1}=0.50$, $M_{\infty}=0.87$.

B-15 Fluctuation in blade force and moment coefficients. $w / c=0.1, \rho_{2} / \rho_{1}=0.75$, $M_{\infty}=0.87$.

B-16 Fluctuation in blade force and moment coefficients. $w / c=0.1, \rho_{2} / \rho_{1}=2.00$, $M_{\infty}=0.87$.

B-17 Fluctuation in blade force and moment coefficients. $w / c=0.2, \rho_{2} / \rho_{1}=0.25$, $M_{\infty}=0.15$.

B-18 Fluctuation in blade force and moment coefficients. $w / c=0.2, \rho_{2} / \rho_{1}=0.50$,

$$
M_{\infty}=0.15 \text {. }
$$

B-19 Fluctuation in blade force and moment coefficients. $w / c=0.2, \rho_{2} / \rho_{1}=0.75$,

$$
M_{\infty}=0.15 \text {. }
$$

B-20 Fluctuation in blade force and moment coefficients. $w / c=0.2, \rho_{2} / \rho_{1}=2.00$,

$$
M_{\infty}=0.15 \text {. }
$$


B-21 Fluctuation in blade force and moment coefficients. $w / c=0.2, \rho_{2} / \rho_{1}=0.25$,

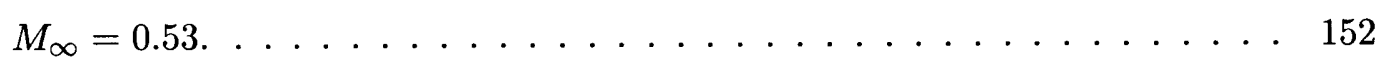

B-22 Fluctuation in blade force and moment coefficients. $w / c=0.2, \rho_{2} / \rho_{1}=0.50$,

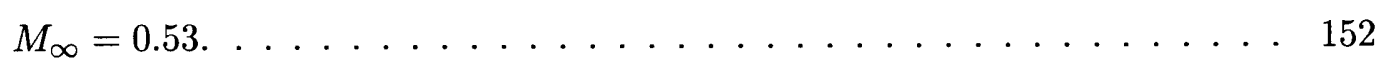

B-23 Fluctuation in blade force and moment coefficients. $w / c=0.2, \rho_{2} / \rho_{1}=0.75$,

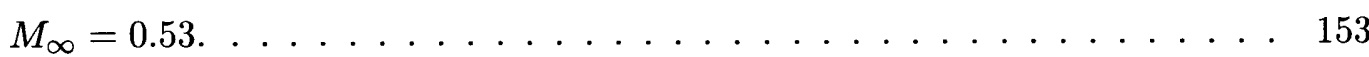

B-24 Fluctuation in blade force and moment coefficients. $w / c=0.2, \rho_{2} / \rho_{1}=2.00$,

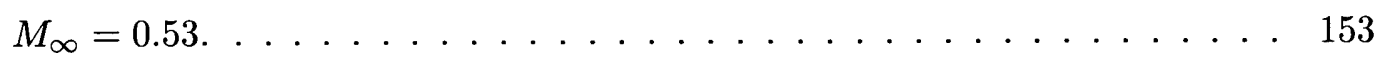

B-25 Fluctuation in blade force and moment coefficients. $w / c=0.2, \rho_{2} / \rho_{1}=0.25$,

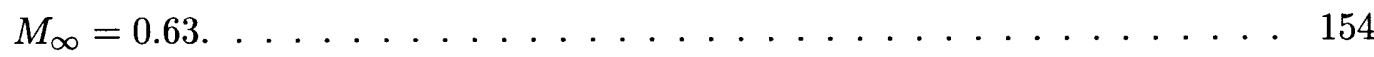

B-26 Fluctuation in blade force and moment coefficients. $w / c=0.2, \rho_{2} / \rho_{1}=0.50$, $M_{\infty}=0.63 \ldots \ldots \ldots \ldots \ldots \ldots \ldots \ldots \ldots$

B-27 Fluctuation in blade force and moment coefficients. $w / c=0.2, \rho_{2} / \rho_{1}=0.75$, $M_{\infty}=0.63$.

B-28 Fluctuation in blade force and moment coefficients. $w / c=0.2, \rho_{2} / \rho_{1}=2.00$, $M_{\infty}=0.63$.

B-29 Fluctuation in blade force and moment coefficients. $w / c=0.2, \rho_{2} / \rho_{1}=0.25$, $M_{\infty}=0.87$.

B-30 Fluctuation in blade force and moment coefficients. $w / c=0.2, \rho_{2} / \rho_{1}=0.50$, $M_{\infty}=0.87$.

B-31 Fluctuation in blade force and moment coefficients. $w / c=0.2, \rho_{2} / \rho_{1}=0.75$, $M_{\infty}=0.87$.

B-32 Fluctuation in blade force and moment coefficients. $w / c=0.2, \rho_{2} / \rho_{1}=2.00$, $M_{\infty}=0.87$.

B-33 Fluctuation in blade force and moment coefficients. $w / c=0.4, \rho_{2} / \rho_{1}=0.25$, $M_{\infty}=0.15$.

B-34 Fluctuation in blade force and moment coefficients. $w / c=0.4, \rho_{2} / \rho_{1}=0.50$, $M_{\infty}=0.15$.

B-35 Fluctuation in blade force and moment coefficients. $w / c=0.4, \rho_{2} / \rho_{1}=0.75$,

$$
M_{\infty}=0.15 \text {. }
$$

B-36 Fluctuation in blade force and moment coefficients. $w / c=0.4, \rho_{2} / \rho_{1}=2.00$,

$$
M_{\infty}=0.15
$$


B-37 Fluctuation in blade force and moment coefficients. $w / c=0.4, \rho_{2} / \rho_{1}=0.25$,

$$
M_{\infty}=0.53 \text {. }
$$

B-38 Fluctuation in blade force and moment coefficients. $w / c=0.4, \rho_{2} / \rho_{1}=0.50$,

$$
M_{\infty}=0.53 \text {. }
$$

B-39 Fluctuation in blade force and moment coefficients. $w / c=0.4, \rho_{2} / \rho_{1}=0.75$,

$$
M_{\infty}=0.53 \text {. }
$$

B-40 Fluctuation in blade force and moment coefficients. $w / c=0.4, \rho_{2} / \rho_{1}=2.00$,

$$
M_{\infty}=0.53 .
$$

B-41 Fluctuation in blade force and moment coefficients. $w / c=0.4, \rho_{2} / \rho_{1}=0.25$,

$$
M_{\infty}=0.63 \text {. }
$$

B-42 Fluctuation in blade force and moment coefficients. $w / c=0.4, \rho_{2} / \rho_{1}=0.50$,

$$
M_{\infty}=0.63 \text {. }
$$

B-43 Fluctuation in blade force and moment coefficients. $w / c=0.4, \rho_{2} / \rho_{1}=0.75$,

$$
M_{\infty}=0.63 .
$$

B-44 Fluctuation in blade force and moment coefficients. $w / c=0.4, \rho_{2} / \rho_{1}=2.00$,

$$
M_{\infty}=0.63 \text {. }
$$

B-45 Fluctuation in blade force and moment coefficients. $w / c=0.4, \rho_{2} / \rho_{1}=0.25$,

$$
M_{\infty}=0.87 \text {. }
$$

B-46 Fluctuation in blade force and moment coefficients. $w / c=0.4, \rho_{2} / \rho_{1}=0.50$,

$$
M_{\infty}=0.87 \text {. }
$$

B-47 Fluctuation in blade force and moment coefficients. $w / c=0.4, \rho_{2} / \rho_{1}=0.75$,

$$
M_{\infty}=0.87 \text {. }
$$

B-48 Fluctuation in blade force and moment coefficients. $w / c=0.4, \rho_{2} / \rho_{1}=2.00$,

$$
M_{\infty}=0.87 \text {. }
$$

B-49 Fluctuation in blade force and moment coefficients. $w / c=1.0, \rho_{2} / \rho_{1}=0.25$,

$$
M_{\infty}=0.15 \text {. }
$$

B-50 Fluctuation in blade force and moment coefficients. $w / c=1.0, \rho_{2} / \rho_{1}=0.50$,

$$
M_{\infty}=0.15 \text {. }
$$

B-51 Fluctuation in blade force and moment coefficients. $w / c=1.0, \rho_{2} / \rho_{1}=0.75$,

$$
M_{\infty}=0.15 \text {. }
$$

B-52 Fluctuation in blade force and moment coefficients. $w / c=1.0, \rho_{2} / \rho_{1}=2.00$,

$$
M_{\infty}=0.15 \text {. }
$$


C-1 Comparison of the $M_{\infty}=0.15$ viscous results and the inviscid results for the maximum fluctuation in the azimuthal force coefficient. . . . . . . . 170

C-2 Comparison of the $M_{\infty}=0.15$ and the $M_{\infty}=0.53$ viscous results for the maximum fluctuation in the azimuthal force coefficient. . . . . . . . . . 170

C-3 Comparison of the $M_{\infty}=0.15$ and the $M_{\infty}=0.63$ viscous results for the maximum fluctuation in the azimuthal force coefficient. . . . . . . . . . 171

C-4 Comparison of the $M_{\infty}=0.15$ and the $M_{\infty}=0.87$ viscous results for the maximum fluctuation in the azimuthal force coefficient. . . . . . . . . 171

C-5 $M_{\infty}=0.15$ viscous result for the maximum fluctuation in the axial force coefficient. . . . . . . . . . . . . . . . . . . . 172

C-6 Comparison of the $M_{\infty}=0.15$ and the $M_{\infty}=0.53$ viscous results for the maximum fluctuation in the axial force coefficient. . . . . . . . . . . . 172

C-7 Comparison of the $M_{\infty}=0.15$ and the $M_{\infty}=0.63$ viscous results for the maximum fluctuation in the axial force coefficient. . . . . . . . . . . . 173

C-8 Comparison of the $M_{\infty}=0.15$ and the $M_{\infty}=0.87$ viscous results for the maximum fluctuation in the axial force coefficient. . . . . . . . . . . 173

C-9 Comparison of the $M_{\infty}=0.15$ viscous results and the inviscid results for the maximum fluctuation in the moment coefficient. . . . . . . . . . . . . . . . 174

C-10 Comparison of the $M_{\infty}=0.15$ and the $M_{\infty}=0.53$ viscous results for the maximum fluctuation in the moment coefficient. . . . . . . . . . . . . 174

C-11 Comparison of the $M_{\infty}=0.15$ and the $M_{\infty}=0.63$ viscous results for the maximum fluctuation in the moment coefficient. . . . . . . . . . . . . . 175

C-12 Comparison of the $M_{\infty}=0.15$ and the $M_{\infty}=0.87$ viscous results for the maximum fluctuation in the moment coefficient. . . . . . . . . . . . 175 


\section{LIST OF TABLES}

2.1 Properties of the LSRC cascade geometry used for the viscous CFD simulations. 48

3.1 Viscous flow simulations. The Reynolds number and Mach number specified in the solver are based on unit blade spacing and unit velocity. . . . . . . . 54

3.2 The time averaged force and moment coefficients for the baseline solutions ranging from $M_{\infty}=0.15$ to $M_{\infty}=0.87 \ldots \ldots \ldots \ldots$

3.3 Comparison of Run 4 shock wave properties with 1D normal shock properties. $\gamma=1.4 \ldots \ldots \ldots \ldots \ldots \ldots \ldots \ldots \ldots \ldots \ldots \ldots \ldots \ldots \ldots \ldots \ldots \ldots \ldots \ldots$

3.4 Flow properties at 3 spanwise locations upstream of a pressure wave in Run

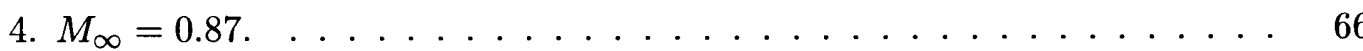

3.5 Vortex shedding frequencies expressed in terms of Strouhal number for each Mach number flow. . . . . . . . . . . . . . . .

4.1 Parametric test variables. $w / c=$ non-dimensional wake width, $\rho_{2} / \rho_{1}=$ maximum density inside wake / free stream density. . . . . . . . . . . . 73

4.2 The location of a density wake at different times during passage through the LSRC cascade blade row. $w / c=0.2, \rho_{2} / \rho_{1}=0.50, M_{\infty}=0.15 . \ldots \ldots$

4.3 Estimated contribution of several sources to the maximum pressure difference across the shock wave. Estimated values are specific to the passage of a density wake of width $0.1 c$ and density ratio $0.5 . M_{\infty}=0.87 \ldots \ldots \ldots$

4.4 Constants in the functional relationships for the maximum fluctuation in the force and moment coefficients (primary response). $M_{\infty}=0.15 \ldots \ldots 9$

4.5 Constants in the functional relationships for the maximum fluctuation in the force and moment coefficients (primary response). $M_{\infty}=0.53 . \ldots . . .90$ 
4.6 Constants in the functional relationships for the maximum fluctuation in the force and moment coefficients (primary response). $M_{\infty}=0.63 . \ldots . . .90$

4.7 Constants in the functional relationships for the maximum fluctuation in the force and moment coefficients (primary response). $M_{\infty}=0.87 \ldots \ldots . . .90$

4.8 Constants in the functional relationships for the maximum fluctuation in the force and moment coefficients (secondary response). Valid for $\rho_{2} / \rho_{1}<1.0$.

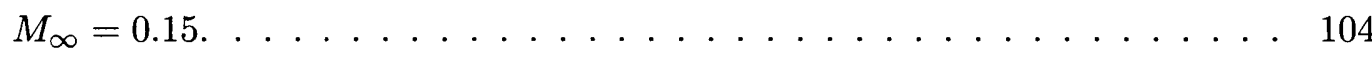

4.9 Constants in the functional relationships for the maximum fluctuation in the force and moment coefficients (secondary response). Valid for $\rho_{2} / \rho_{1}<1.0$.

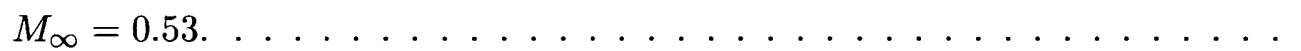

4.10 Constants in the functional relationships for the maximum fluctuation in the force and moment coefficients (secondary response). Valid for $\rho_{2} / \rho_{1}<1.0$.

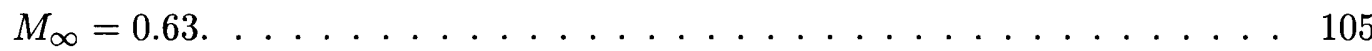

4.11 Constants in the functional relationships for the maximum fluctuation in the force and moment coefficients (secondary response). Valid for $\rho_{2} / \rho_{1}<1.0$.

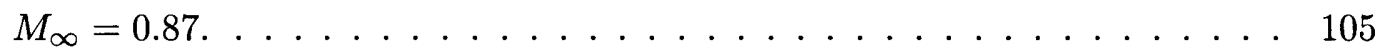

5.1 Properties of the NACA4F cascade geometry used for the inviscid CFD tests. 118

5.2 Cascade model parameters used to determine the flat plate force and moment coefficient fluctuation during passage of a density wake of width $0.1 c$ and density ratio $0.5 \ldots \ldots \ldots \ldots \ldots \ldots \ldots$ 


\section{Symbols}

$c$

$d$

$e$

$h$

$k$

Blade chord

Spacing of counterrotating vortices

Energy

Blade spacing

Height of counterrotating vortices above flat plate

$\sqrt{-1}$

Index of summation

Moment arm length

Upstream extent of flat plate pressure distribution

Downstream extent of flat plate pressure distribution

Static pressure

Distance from line vortex

Time

Velocity in axial direction

Velocity in azimuthal direction

Fluid flux velocity in azimuthal direction

$w \quad$ Wake width

Induced velocity

Axial direction coordinate

Azimuthal direction coordinate 


\begin{tabular}{|c|c|}
\hline$y^{+}$ & Boundary layer coordinate $\left(\sqrt{\tau_{w} / \rho y}\right) / \nu$ \\
\hline$A_{\text {eff }}$ & Effective area used in cascade model \\
\hline$C_{\mu}$ & Turbulence model constant \\
\hline$C_{1}$ & Turbulence model constant \\
\hline$C_{2}$ & Turbulence model constant \\
\hline$C f$ & Wall skin friction coefficient \\
\hline$C m$ & Moment coefficient about mid chord (positive clockwise) \\
\hline$C p$ & Pressure coefficient \\
\hline$\Delta C p_{s}$ & Static pressure rise coefficient across the shock wave \\
\hline$C x$ & Axial force coefficient \\
\hline$C y$ & Azimuthal force coefficient \\
\hline$K$ & Constant of proportionality in cascade model \\
\hline$M$ & Mach number \\
\hline$N$ & Number of discrete vortex panels \\
\hline$R$ & Gas constant \\
\hline$R e$ & Reynolds number \\
\hline$S t$ & Strouhal number \\
\hline$T$ & Flat plate cascade spacing \\
\hline$U$ & Total velocity \\
\hline$U_{R}$ & Rotor tip speed \\
\hline$U_{\text {edge }}$ & Boundary layer edge velocity at blade trailing edge \\
\hline$\alpha$ & Angle between flow and flat plate \\
\hline$\beta_{1}$ & Flat plate stagger angle \\
\hline$\delta^{*}$ & Boundary layer displacement thickness \\
\hline$\eta$ & Coordinate normal to flat plate \\
\hline$\gamma$ & Ratio of specific heats $(\gamma=1.4)$ \\
\hline$\lambda$ & Position of density discontinuity \\
\hline \multirow[t]{2}{*}{$\mu$} & Reduced frequency $(f c / U)$ \\
\hline & Viscosity \\
\hline$\nu$ & Kinematic viscosity \\
\hline$\omega$ & Vorticity \\
\hline$\rho$ & Density \\
\hline
\end{tabular}




$\begin{array}{ll}\rho^{*} & \text { Density parameter }\left(\rho_{2}-\rho_{1}\right) /\left(\rho_{2}+\rho_{1}\right) \\ \Psi_{s-s} & \text { Static to static pressure rise coefficient } \\ \xi & \text { Coordinate along flat plate } \\ \sigma & \text { Flat plate cascade space-chord ratio }(T / c) \\ \sigma_{\kappa} & \text { Turbulence model constant } \\ \sigma_{\epsilon} & \text { Turbulence model constant } \\ \theta & \text { Boundary layer momentum thickness } \\ \tau & \text { Non dimensional time } \\ \tau_{w} & \text { Wall shear stress } \\ \vartheta & \text { Blade trailing edge thickness } \\ \zeta & \text { Location of any point in the complex plane }(\zeta=\xi+i \eta) \\ \Gamma & \text { Circulation strength } \\ \Gamma_{A} & \text { Circulation strength of counterrotating vortex } A \\ \Gamma_{B} & \text { Circulation strength of counterrotating vortex } B \\ \Gamma_{k} & \text { Circulation strength of vortex panel } k \\ \Gamma_{m a x} & \text { Maximum circulation strength of counterrotating vortices }\end{array}$

\section{Subscripts}

1 Free stream or value outside density wake

2 Value inside density wake

Behind density discontinuity for Marble's analysis

$\infty \quad$ Free stream value or total value

\section{Operators and Modifiers}

$\begin{array}{ll}\widetilde{()} & \text { Non-dimensionalized quantity } \\ \Delta & \text { Difference operator } \\ \nabla & \text { Gradient operator }\end{array}$




\section{Acronyms}

$\begin{array}{ll}\text { HCF } & \text { High Cycle Fatigue } \\ \text { CFD } & \text { Computational Fluid Dynamics } \\ \text { DFT } & \text { Discrete Fourier Transform }\end{array}$




\section{Chapter 1}

\section{INTRODUCTION}

\section{$1.1 \quad$ Background}

Increased operational requirements and increased thrust to weight ratios have led to higher mean and fluctuating stresses in components of modern turbomachinery. This has increased the likelihood of encountering high cycle fatigue (HCF) failure in fan, compressor and turbine blades. The U.S. Air Force in particular claims $50 \%$ of their total irrecoverable in-flight engine shutdowns can be traced to HCF failure. This clearly places a huge burden on maintaining a mission ready force and consequently the prevention of (HCF) failure in turbomachinery components has become an increasingly important issue. Furthermore at a recent HCF workshop held at the MIT Gas Turbine Laboratory [27] it was noted that

“...forced blade response is not currently predictable, and structural design and analysis for high cycle fatigue situations have not advanced beyond the early concepts of the fatigue limit, the Goodman diagram and Miner's rule.".

The HCF "problem free" engine operation requires technology advances in four key technological areas [27]:

- Aerodynamic vibration forcing function prediction.

- Structural analysis and modeling tools.

- Measurement and analysis tools. 
- Material characterization.

This list of technological areas clearly indicates the multidisciplinary nature of the HCF problem. The large number of parameters and the wide occurrence of HCF producing conditions over the engine operating regime imply that structural integrity must be evaluated in an extremely large number of situations. An additional implication is that it is difficult to extract general guidelines for high cycle fatigue prevention because of the high dimensionality of the parameter space that must be explored [27]. Further complexity is introduced by the diversity of local phenomena, e.g. tip leakage flows, unsteady shock motion and local separation that are characteristic of turbomachinery flows. This is a major reason why $\mathrm{HCF}$ continues to be a challenging problem.

The first item above, namely the aerodynamic forcing functions ${ }^{1}$ are not well predicted for off-design conditions particularly at high loading. Several forced vibration "sources" exist in turbomachinery. In particular viscous wakes from upstream blade rows and potential flow interactions due to rotor-stator interaction have received a lot of attention (Kemp and Sears [11], Kerrebrock and Mikolajczak [12], Manwaring and Wisler [15], Valkov [23]). These studies have been primarily concerned with compressor performance however and the corresponding effects on unsteady blade loading have not been considered.

The purpose of the current research is to characterize the unsteady aerodynamic forces and moments induced in turbomachinery cascade blade rows by convecting density wakes. Density wakes have been recently identified as a possible new source for high cycle fatigue failure. Density wakes can enter the engine from ground ingested hot air, steam ingestion during carrier launches and exhaust gas ingestion from forward firing weapons [18]. The difference in temperature between the blades and the surrounding fluid can also generate density gradients particularly in the downstream wakes of the blades. Incomplete or nonuniform combustion also introduces density gradients to the flow entering turbines.

The first study of density wake induced forces and moments was conducted by Mar-

\footnotetext{
${ }^{1}$ Note that forced vibrations are distinct from self excited vibrations, e.g. blade flutter and dynamic stall.
} 


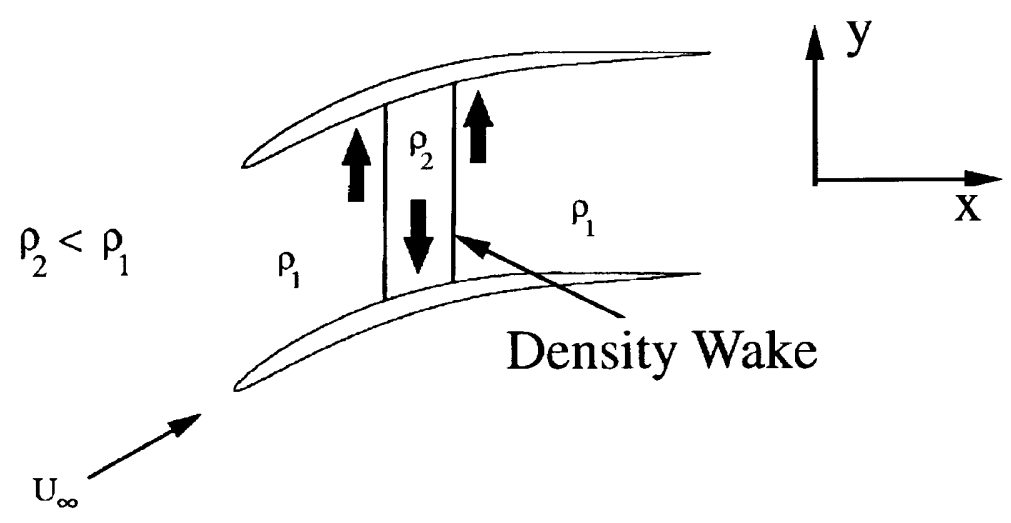

Figure 1-1: Density wake convecting through a compressor blade row.

ble [16] for a flat plate airfoil. The density wake induced forces in cascade compressor blade rows was later investigated by Ramer [19] for inviscid incompressible flows.

In the following sections a physical description of the origin of density wake induced blade forces is described. This is followed by a theoretical background which includes the derivation of non-dimensional scaling relationships. Marble's linearized potential flow results are presented here. The results obtained from inviscid flow simulations [19] are presented next. The questions posed in the present research, the contributions from the thesis and the technical approach is then described. Finally an overall description of the thesis organization is detailed.

\subsection{Physical Origin of Unsteadiness}

An analogy to the passage of a density wake through a compressor blade row can be found in the atmosphere; low density (high temperature) air rises to higher altitudes where the pressure is lower and remains there because of force equilibrium. Similarly, high density (low temperature) air sinks to regions of high pressure nearer to the earth.

Now consider the passage of a density wake through a cascade blade row as illustrated in Figure 1-1. Assume the wake has a lower density than the free stream density. As the 
wake moves through the cascade, the low density fluid migrates towards the suction side of the blades by the action of centrifugal forces. To satisfy mass conservation the surrounding higher density fluid is subsequently displaced toward the pressure side of the blade. This relative motion of low and high density fluids generates a pair of counterrotating vortices in the blade passage. The low density fluid directed toward the blade suction surface and the associated counterrotating vortices convect through the blade passage together with the density wake.

The blade pressure distribution is influenced by the impact of the low density fluid on the blade surface. The blade force and moment coefficients therefore change with time during passage of the density wake. These force and moment fluctuations are the major topic in all subsequent sections. First however the theoretical basis for the generation of vorticity in the blade passage is discussed. This is followed by derivation of non-dimensional scaling relationships.

\subsection{Theoretical Background: Marble's Linearized Analysis}

Vorticity is generated by the interaction of the flow density gradient with the flow pressure gradient. For flow situations where the density gradient and the pressure gradients are aligned no vorticity can be generated ${ }^{2}$. For the case of a density wake convecting through a compressor blade row as shown in Figure 1-1 however the density gradients and pressure gradients are misaligned by almost 90 degrees. This misalignment allows for vorticity production in the flow ${ }^{3}$. For a continuous density distribution in two-dimensional, inviscid, incompressible flow this vorticity satisfies the linearized relation,

$$
\left(\frac{\partial}{\partial t}+u \frac{\partial}{\partial x}\right) \omega=\frac{1}{\rho^{2}} \nabla p \times \nabla \rho
$$

\footnotetext{
${ }^{2}$ Vorticity may be generated by other sources however, e.g. by viscous and non-conservative body forces.

${ }^{3}$ The generation of vorticity due to the misalignment of density gradients and pressure gradients is often referred to as "baroclinic torque".
} 


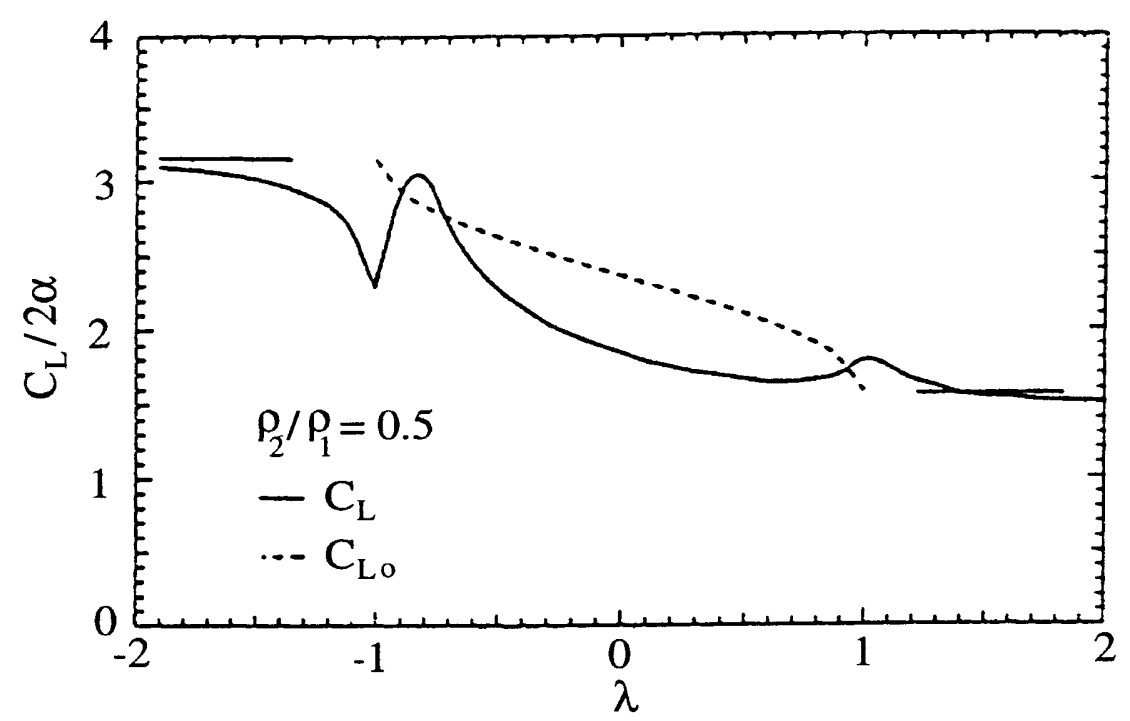

[a]

Figure 1-2: Lift coefficient fluctuation during passage of a density discontinuity over a flat plate. $\lambda$ is the position of the density discontinuity as it convects along the flat plate. The flat plate lies between $|\lambda| \leq 1$.

If the density gradient $(\nabla \rho)$ is large (zeroth order), the convected vorticity $\omega$ is of the same order as the pressure field [16].

The first study of density gradients as a source of flow unsteadiness was conducted by Marble [16]. He performed a linearized potential flow analysis for a flat plate at an angle of attack $\alpha$ encountering a density discontinuity. If the fluid is treated as incompressible and the velocity disturbances caused by the airfoil are small compared to the free stream velocity, the density field can be expressed as $\rho(x-u t, y)[16]$. The results of Marble's analysis are shown in Figure 1-2 and Figure 1-3. In these Figures $\lambda$ is the position of the density discontinuity as it convects over the flat plate. The flat plate lies between $|\lambda| \leq 1$.

The dotted lines correspond to the quasi-steady results while the solid lines correspond to the unsteady results. Initially the effect of the density discontinuity is to reduce the local lift. This is a consequence of a downwash velocity field which precedes the arrival of the discontinuity. This is followed by a rapid rise in lift as the discontinuity convects over 


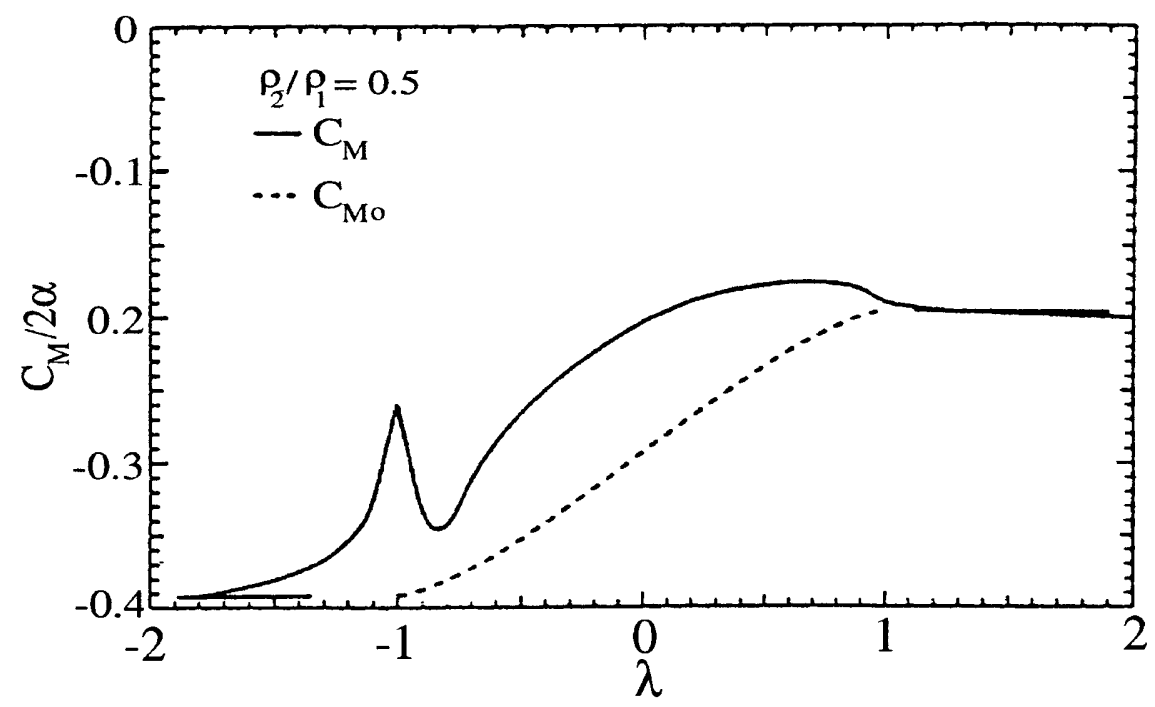

[b]

Figure 1-3: Moment coefficient fluctuation during passage of a density discontinuity over a flat plate. $\lambda$ is the position of density discontinuity as it convects along the flat plate. The flat plate lies between $|\lambda| \leq 1$.

the leading edge. This is caused by an upwash velocity field behind the discontinuity. A gradual relaxation of the perturbation occurs as the density discontinuity convects further downstream. The final steady lift scales with the ratio of the density across the discontinuity. The moment coefficient shown in Figure 1-3 also reflects these events in local loading.

Marble's linearized analysis provides a basic understanding of the parameters involved in this problem. In particular the density parameter $\rho^{*}$,

$$
\rho^{*}=\frac{\rho_{2}-\rho_{1}}{\rho_{2}+\rho_{1}}
$$

is shown to be a key parameter in the unsteady loading. Re-writing Equation 1.1 using the density parameter $\rho^{*}$ and the non-dimensionalized vorticity $\tilde{\omega}=\omega c / U_{\infty}$ gives, 


$$
\frac{D \tilde{\omega}}{D \tilde{\tau}}=\frac{c^{2}}{w h} \rho^{*}\left[\frac{1}{\tilde{\rho}^{2}}\left(\widetilde{\nabla}_{w} \tilde{\rho} \times \widetilde{\nabla}_{h} \tilde{p}\right)\right]
$$

where,

$$
\begin{aligned}
\tilde{p} & =\frac{p}{\left(\rho_{2}-\rho_{1}\right) U_{\infty}^{2}} \\
\tau & =\frac{U_{\infty}}{c} t \\
\tilde{\rho} & =\frac{\rho}{\rho_{1}+\rho_{2}} \\
\tilde{\nabla}_{w} & =w \nabla \\
\tilde{\nabla}_{h} & =h \nabla
\end{aligned}
$$

Equation 1.3 suggests the non-dimensional wake width $w / c$ and non-dimensional blade spacing $h / c$ to be additional key parameters.

\subsection{Inviscid Flow Simulations}

The non-dimensional parameters determined above $\left(\rho^{*}, w / c\right.$ and $\left.h / c\right)$ were used by Ramer [19] in the design of two-dimensional, inviscid incompressible flow simulations of convecting density wakes ${ }^{4}$. A single density wake with a sinusoidal density variation from free stream density $\rho_{1}$ to a peak inner density $\rho_{2}$ was used in these simulations.

The inviscid results indicated a localized reduction in pressure difference $\Delta C p$ across

\footnotetext{
${ }^{4}$ Note the incompressible flow assumption does not preclude the possibility of regions with non-uniform density. These regions are simply convected with the flow.
} 


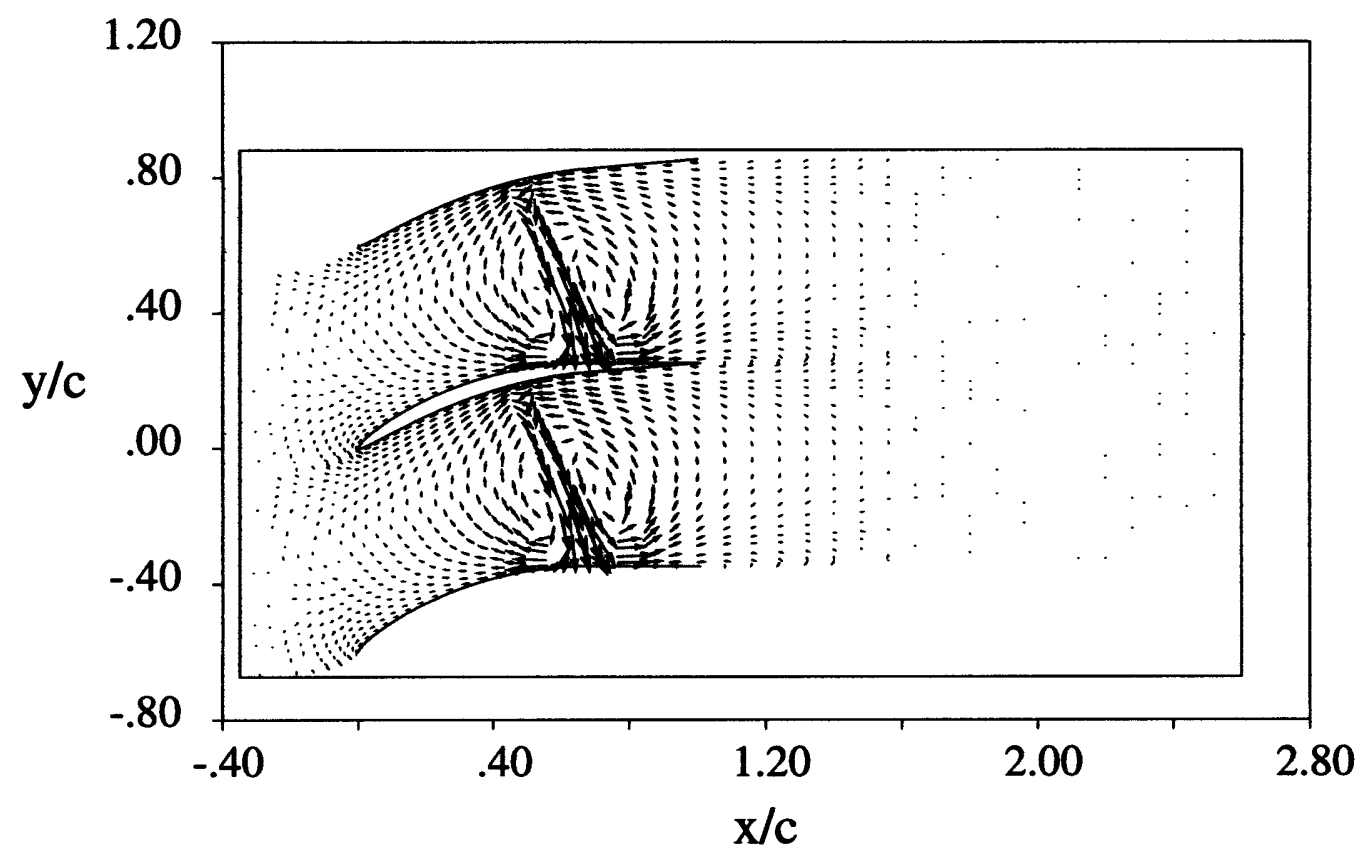

Figure 1-4: Perturbation velocity vectors during passage of a density wake of width $0.2 c$ and density ratio 0.5 through the NACA4F blade row. The flow is inviscid and incompressible. $\tau=0.65$.

the blade row during passage of the density wakes. This local reduction in $\Delta C p$ is a result of the low density fluid directed toward the blade suction surface ${ }^{5}$. The perturbation velocity vectors plotted in Figure 1-4 clearly indicate this fluid motion and the associated counterrotating vortex pairs ${ }^{6}$.

The corresponding fluctuation in the blade azimuthal force coefficient $C y$ and moment coefficient $C m$ (about the blade mid chord) is shown in Figure 1-5 for a range of density wake widths and density ratios. Initially a reduction in the azimuthal force occurs as the density wake convects over the front half of the blade. This is followed by a gradual return to steady state as the density wake leaves the blade trailing edge. Similarly an increase in the counterclockwise moment occurs as the density wake convects over the front half of the blade. As the density wake passes the blade mid chord, the moment decreases back towards steady state. The shape of these force and moment profiles are roughly common over the

\footnotetext{
${ }^{5}$ Further discussion can be found in Chapter 4.

${ }^{6}$ It can be argued that the induced velocity field of the counterrotating vortices direct the density wake fluid to the blade suction surface. No clear distinction can be made between cause and effect however. Both events (fluid flow and counterrotating vortices) occur simultaneously.
} 

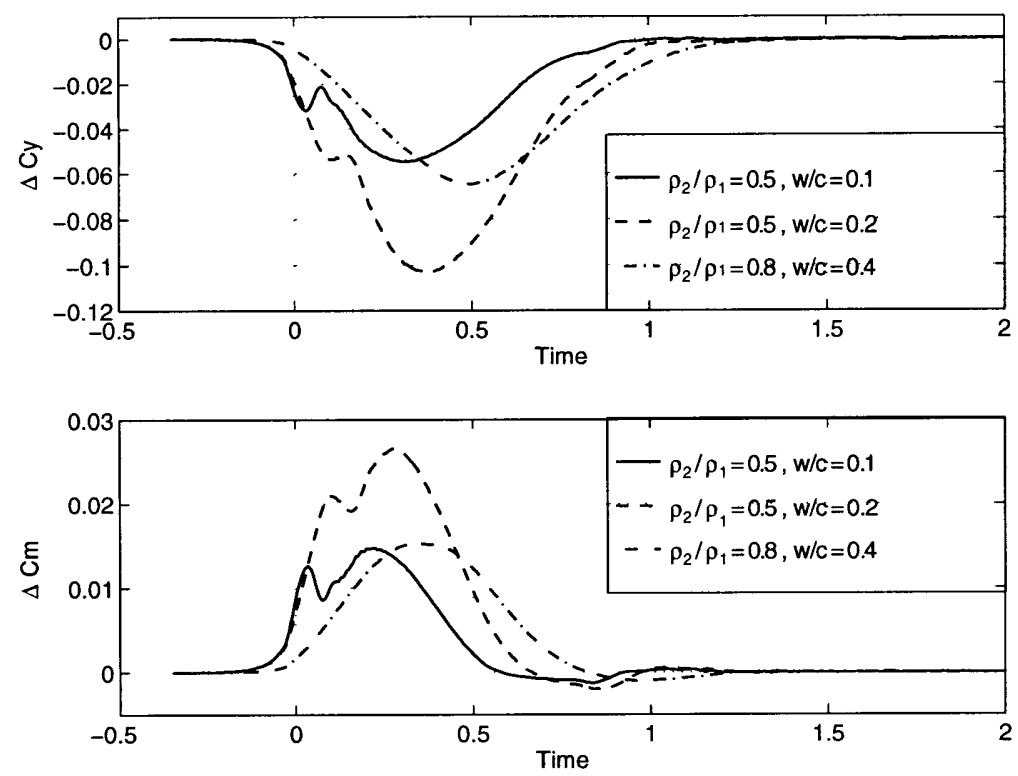

Figure 1-5: Fluctuation in (a) azimuthal force coefficient and (b) moment coefficient (positive counter-clockwise about the mid-chord) during passage of density wakes. $\Delta C y=$ $C y_{\text {max }}-C y_{\text {mean }}, \Delta C m=C m_{\text {max }}-C m_{\text {mean }}$ where $C y_{\text {mean }}=0.75, C m_{\text {mean }}=$ -0.13 . Reproduced from Ramer.

range of density wake widths, $0.1 \leq w / c \leq 0.4$, and density ratio's $0.25 \leq \rho_{2} / \rho_{1} \leq 2.0$ studied. The maximum change in the force and moment coefficients as functions of wake width and Marbles density parameter $\rho^{*}$ are plotted in Figure 1-6 and Figure 1-7.

The amplitude of the maximum fluctuations in the force and moment coefficients were found to scale linearly for small wake widths $w / c$ and small density parameter $\rho^{*}$. This scaling is given by,

$$
\begin{gathered}
\frac{C y_{\text {max }}-C y_{\text {mean }}}{C y_{\text {mean }}}=2.19\left(\frac{w}{c}\right) \rho^{*} \\
\frac{C m_{\text {max }}-C m_{\text {mean }}}{C m_{\text {mean }}}=-3.05\left(\frac{w}{c}\right) \rho^{*}
\end{gathered}
$$




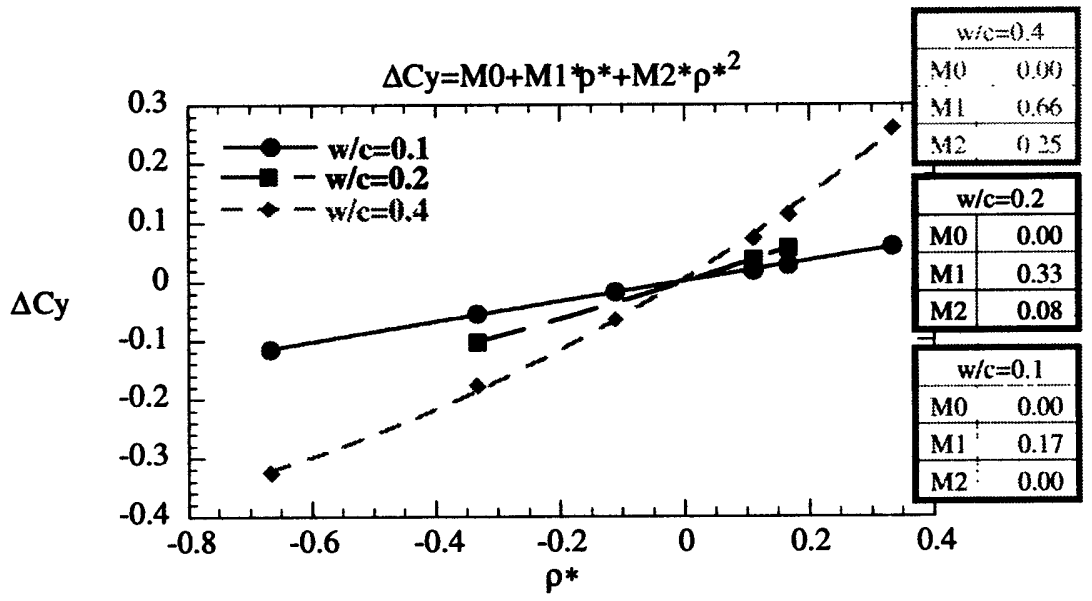

Figure 1-6: Maximum fluctuation in the azimuthal force coefficient (from steady state) during passage of density wakes through the NACA4F blade row. $\Delta C y=\left(C y_{\max }-\right.$ $\left.C y_{\text {mean }}\right) / C y_{\text {mean }}$. Reproduced from Ramer.

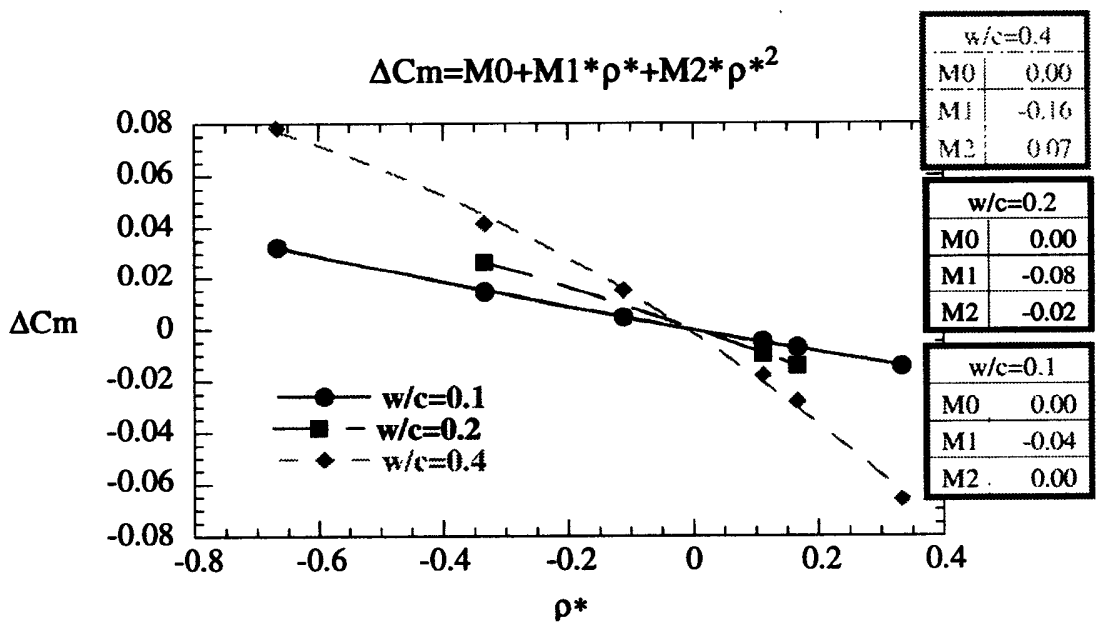

Figure 1-7: Maximum fluctuation in moment coefficient (from steady state) during passage of density wakes through the NACA4F blade row. $\Delta C y=\left(C y_{\text {max }}-C y_{\text {mean }}\right) / C y_{\text {mean }}$. Reproduced from Ramer. 


\subsubsection{Conclusions Based on Inviscid Results}

1. The controlling flow feature responsible for the density wake induced blade force and moment fluctuations in inviscid incompressible flows is the flow of density wake fluid by the action of centrifugal forces. During passage of a low density wake the wake fluid is directed toward the blade suction surface. This low density fluid reduces the blade force coefficient and increases the counter-clockwise moment coefficient. The opposite is true for a wake with higher density than free stream. In this case the density wake fluid is directed toward the blade pressure surface.

2. The shape of the force and moment coefficient fluctuations are common over the range of density wake widths $0.1 \leq w / c \leq 0.4$ and density ratios $0.25 \leq \rho_{2} / \rho_{1} \leq 2.0$ considered.

3. Parametric studies show the amplitude of the maximum fluctuation in blade force and moment coefficients to have the following functional relationship:

$$
\begin{aligned}
& \Delta C y=f\left(w / c, \rho^{*}, C y(\text { mean })\right) \\
& \Delta C m=f\left(w / c, \rho^{*}, C m(\text { mean })\right)
\end{aligned}
$$

The effect of blade spacing $h / c$ is included in $C y($ mean) and $C m($ mean).

4. For $w / c \leq 0.2$ and $-0.2 \leq \rho^{*} \leq 0.2, \Delta C y$ and $\Delta C m$ scale linearly. Increasing non-linearity is observed for larger wake widths and density ratios.

For further discussion of the inviscid results see Ramer [19], Ramer [21] and Wijesinghe [24]. Details of the inviscid flow solver and computational grid can also be found in Ramer [19].

\subsection{Questions Posed by the Current Research}

Prior research on density wake induced blade force and moment fluctuations have been restricted to the case of inviscid incompressible background flows. These assumptions do not hold near blade surfaces and at high speeds typical of turbomachinery fans and compressors where boundary layers and blade passage shock waves could be significant. Furthermore it is unclear whether the functional relationships and parametric variables governing density 
wake induced force and moment fluctuations in inviscid incompressible flows are applicable to viscous compressible flow environments. The following questions are posed to address this problem:

1. What are the additional controlling fluid dynamic features responsible for the blade force and moment fluctuations in viscous compressible background flows?

2. What additional scaling parameters (if any) besides density wake width and density parameter $\rho^{*}$ are required to quantify the blade force and moment fluctuations for a given Mach number?

3. What are the parametric trends in force and moment fluctuations with increasing free stream Mach number?

The answers to these questions will help characterize the density wake induced forces and moments to a more broader realistic range of flow environments.

A key obstacle to HCF prevention alluded to earlier is the difficulty to formulate general guidelines which can provide a bound on the maximum fluctuating forces and moments. This is due to the high dimensionality of the parameter space that must be investigated. A simple model which can accurately predict this boundary economically with low expenditure in time and cost will be of value to the design process. To help initiate the development of such a model the following questions are posed:

1. Can a simple physical flow model be developed from the CFD results to determine the trends in the blade force and moment fluctuations ?

2. If so can this model be used to predict trends in force and moment fluctuations for a wider range of cascade geometries and density wake properties?

\subsection{Technical Approach}

Computational fluid dynamics (CFD) is the "tool" used in this research to investigate the forces and moments induced by convecting density wakes in viscous compressible back- 
ground flows. CFD is a relatively inexpensive and convenient method to investigate flow phenomena compared to experimental investigations in wind tunnels. In particular the flow field properties at any location in the computational domain can be conveniently determined and an overall "picture" of the flow can be generated to help identify specific flow features. The flow geometry and free stream conditions are also easily changed within a CFD simulation compared to an experimental facility where arbitrary changes in flow geometry are generally not feasible.

The use of CFD is constrained by available computational resources however. This research has therefore been limited to two-dimensional unsteady flows with a single compressor blade row geometry. While turbine blades are subjected to larger density nonuniformities (due to hot-streaks from the combustor and from blade cooling), compressor blades are considered here since they are more susceptible to HCF failure.

The density wakes considered convect along the axial direction and have density gradients directed only in the axial direction. Discussion is focused on low density wakes (wake densities lower than free stream density) which are more common in compressor blade passages. To help isolate individual flow features a single density wake is convected through the blade passage in all simulations. The density variation inside the density wake is specified to be sinusoidal. This variation is considered to be a representative case.

To address the issue of the feasibility of a physical model to investigate the wide parameter space of density wake - cascade blade row interactions a simple cascade model is developed. The model uses a combination of singularity solutions and a proportional constant determined from the inviscid CFD results. 


\subsection{Thesis Contributions}

The aim of this research has been to contribute towards the "aerodynamic vibration forcing prediction" aspect of the high cycle fatigue problem. In this regard convecting density wakes were identified as a possible sources of high cycle fatigue failure. The density wake induced blade force and moment fluctuations were characterized for viscous compressible background flows with flow Mach numbers ranging from $M_{\infty}=0.15$ to $M_{\infty}=0.87$. The important contributions from this thesis can be listed as follows.

- The magnitude of the force and moment fluctuations in viscous compressible flows were quantified for,

1. Density wake widths $w / c=0.1,0.2,0.4,1.0$

2. Density ratios $\rho_{2} / \rho_{1}=0.25,0.50,0.75,2.00$.

3. Flow Mach numbers $M_{\infty}=0.15,0.53,0.63,0.87$.

- The force and moment fluctuation magnitudes in viscous compressible flow are found to scale with (1) the non-dimensional density wake width $w / c$ and (2) Marble's density parameter $\rho^{*}$ for a given Mach number. The force and moment fluctuation mgnitudes also increase with flow Mach number. The maximum fluctuation in the azimuthal force coefficient in particular was found to scale with the Prandtl-Glauert compressibility factor $\sqrt{1-M_{\infty}^{2}}$ for small density wake widths $(w / c=0.1)$. The PrandtlGlauert factor does not adequately scale the axial force coefficient or the moment coefficient however. Additional compressibility scaling relations are required for these coefficients.

- The baseline viscous compressible background solutions obtained prior to introducing density wakes have uncovered "self-excited" blade force and moment fluctuations due to periodic vortex shedding at the blade trailing edge. The vortex shedding induced force and moment fluctuations have amplitudes up to $\pm 13 \%$ from the time averaged mean values. This is a possible additional source for HCF failure.

- The density wake - boundary layer interaction was found to generate a separation bubble on the blade suction surface which causes additional blade force and moment fluctuations. The amplitude of these additional fluctuations scale with the maximum 
fluctuation in the suction surface separation point which in turn scales with (1) the density wake width $w / c$ and (2) the density ratio $\rho_{2} / \rho_{1}$. The separation bubble is also found to disrupt vortex shedding at the blade trailing edge for 2-3 convective time scale units after the density wake leaves the blade trailing edge.

- A cascade flow model was developed to help establish trends in the force and moment fluctuations with changes in density wake properties and cascade geometry in a time efficient manner. The model can also be used to predict a bound on the maximum force and moment fluctuations.

\subsection{Thesis Organization}

Chapter 2 details the viscous flow solver developed by Hoying [9] which is used for the viscous simulations. A brief summary of the numerical scheme and the computational grid is presented here together with a description of the boundary conditions.

The baseline solutions obtained prior to the introduction of density wakes is then described separately in Chapter 3.

Chapter 4 details the blade force and moment fluctuations induced by convected density wakes for several free stream Mach numbers. The non-dimensional wake width $w / c$ and density ratio $\rho_{2} / \rho_{1}$ are used as variables for parametric tests to determine the trends in the maximum force and moment fluctuations.

Chapter 5 presents the cascade flow model developed to determine parametric trends in the blade force and moment fluctuations with changes in the density wake properties and cascade geometry. The model assumptions, governing equations and results are presented in this Chapter.

Chapter 6 presents the conclusions from this research and and lists topics for further research. 


\section{Chapter 2}

\section{VisCOUS Flow SOLVER}

A two-dimensional, time-accurate, explicit Navier-Stokes flow solver developed by Hoy-

ing [9] is used for all the viscous compressible flow simulations. The aim of this chapter is to briefly highlight the main features of the flow solver and to detail the computational grid and boundary conditions used to obtain converged solutions. The compressor cascade properties and the imposed shape of the density wake profiles is also detailed. The characteristic variables used to non-dimensionalize the results is also described.

\subsection{Features of The Viscous Flow Solver}

The key features of the Navier-Stokes flow solver are summarized below.

1. Finite difference discretization.

2. Fourth-order spatial accuracy and third-order temporal accuracy.

3. Dispersion Relation Preserving (DRP) scheme (Tam and Web [22]).

4. $\kappa-\epsilon$ turbulence model.

5. Wall functions (Chieng and Launder [3]).

6. Non-reflecting inlet and exit boundary conditions (Giles [5]).

The Dispersion Relation Preserving scheme used in this solver has the advantage of optimizing the dissipation and dispersion characteristics of the finite difference method over 
a large number of wavelengths (than say a four-stage Runga-Kutta scheme). The resulting high-order scheme also reduces the computational cost by requiring fewer grid points. As is the case with most high-order methods, the presence of high frequency waves in the solution is of great concern. The scheme is noted as being able to capture high frequency waves to an extent that unwanted waves will remain in the solution. Artificial damping based on the fourth derivative of pressure at each node however was found to be effective in removing the high-frequency waves without creating excessive damping of the desired waves. Specific implementation details are described in Hoying [9].

A standard two equation $\kappa-\epsilon$ turbulence model was implemented in the solver using the following model constants,

$$
C_{\mu}=0.09, C_{1}=1.44, C_{2}=1.92, \sigma_{\kappa}=1.0, \sigma_{\epsilon}=1.3
$$

These constants as proposed by Launder and Spalding [13] are appropriate for free shear flows and flows near solid boundaries. A two-stage MacCormack Predictor-Corrector method is chosen to integrate the $\kappa^{-\epsilon}$ model equations. The flow equations and the $\kappa^{-}$ $\epsilon$ model equations are advanced in time with the same time-step.

Additional computational savings are achieved by using wall functions to model the inner portion of the blade boundary layers. Wall functions allow the first near wall grid point to be located at $y^{+} \approx 30-150$. This results in a considerable saving of the number of near wall grid points. The wall functions calculate the wall shear stress and therefore a zero slip velocity at the wall cannot be specified as a boundary condition. A finite slip velocity is specified instead such that the correct wall shear stress is achieved ${ }^{1}$. An adiabatic wall boundary condition is also applied at the blade surface.

The computational domain used in the solver is shown schematically in Figure 2-1. The flow upstream and downstream of the blade row is assumed to be governed by the Euler equations. The flow in the blade passages surrounding the blades is assumed to be

\footnotetext{
${ }^{1}$ See Hoying for more details.
} 


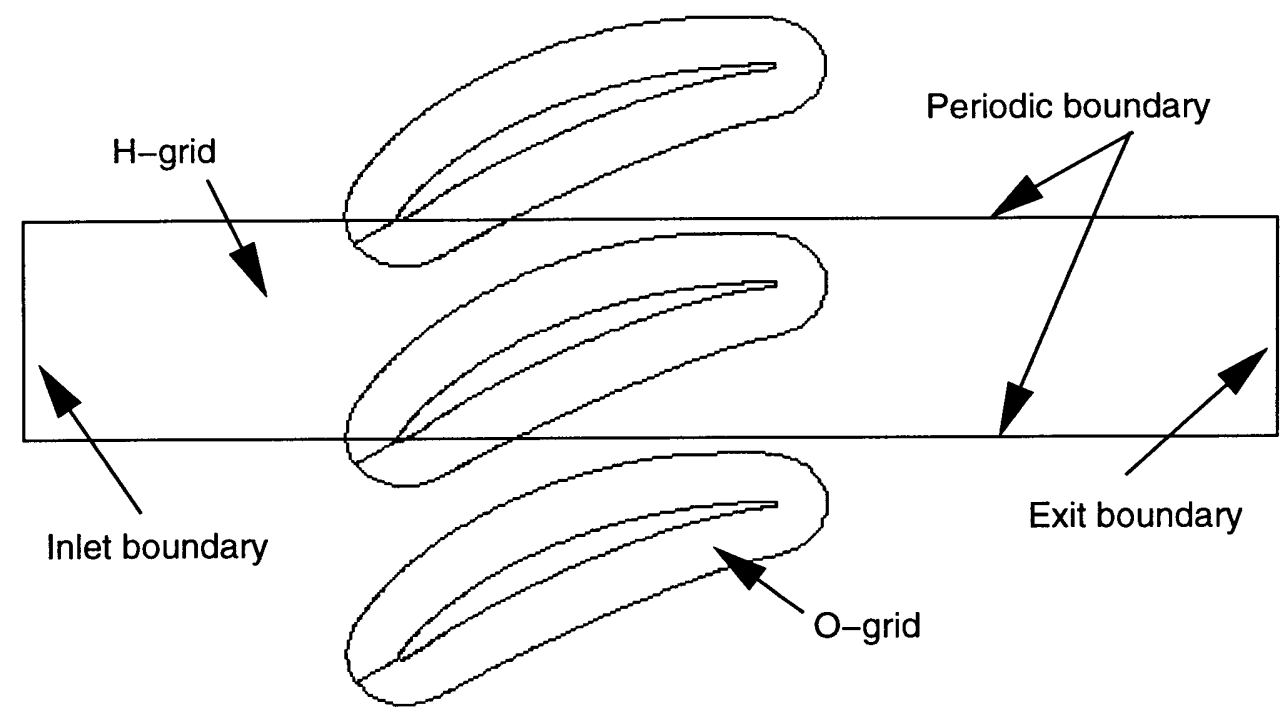

Figure 2-1: Schematic of the computational domain and boundary conditions.

governed by the Navier-Stokes equations. This division helps to reduce the computational time since the solution of the more expensive Navier-Stokes equations is confined to a physically smaller region of the flow.

Non-reflecting boundary conditions developed by Giles [5] are implemented at the inlet and exit boundaries of the computational domain. The aim of these boundary conditions are to allow all out-going modes of the solution to propagate through the inlet and exit boundaries without reflecting back into the computational domain. Periodic boundary conditions are employed along the side boundaries of the computational domain parallel to the axial flow direction. These boundary conditions are shown in Figure 2-1.

\subsection{Computational Grid}

The computational domain used in the solver consists of overlapping $\mathrm{H}$ and $\mathrm{O}$ grids as shown schematically in Figure 2-1. The H-grid has uniform spacing throughout the computational domain with a cell aspect ratio of 1.0. The orthogonality of the H-grid simplifies the evaluation of numerical derivatives as well as the $\mathrm{H}$ to $\mathrm{O}$ grid interpolation coefficients. The O-grid was generated using an elliptic partial differential equation method developed 


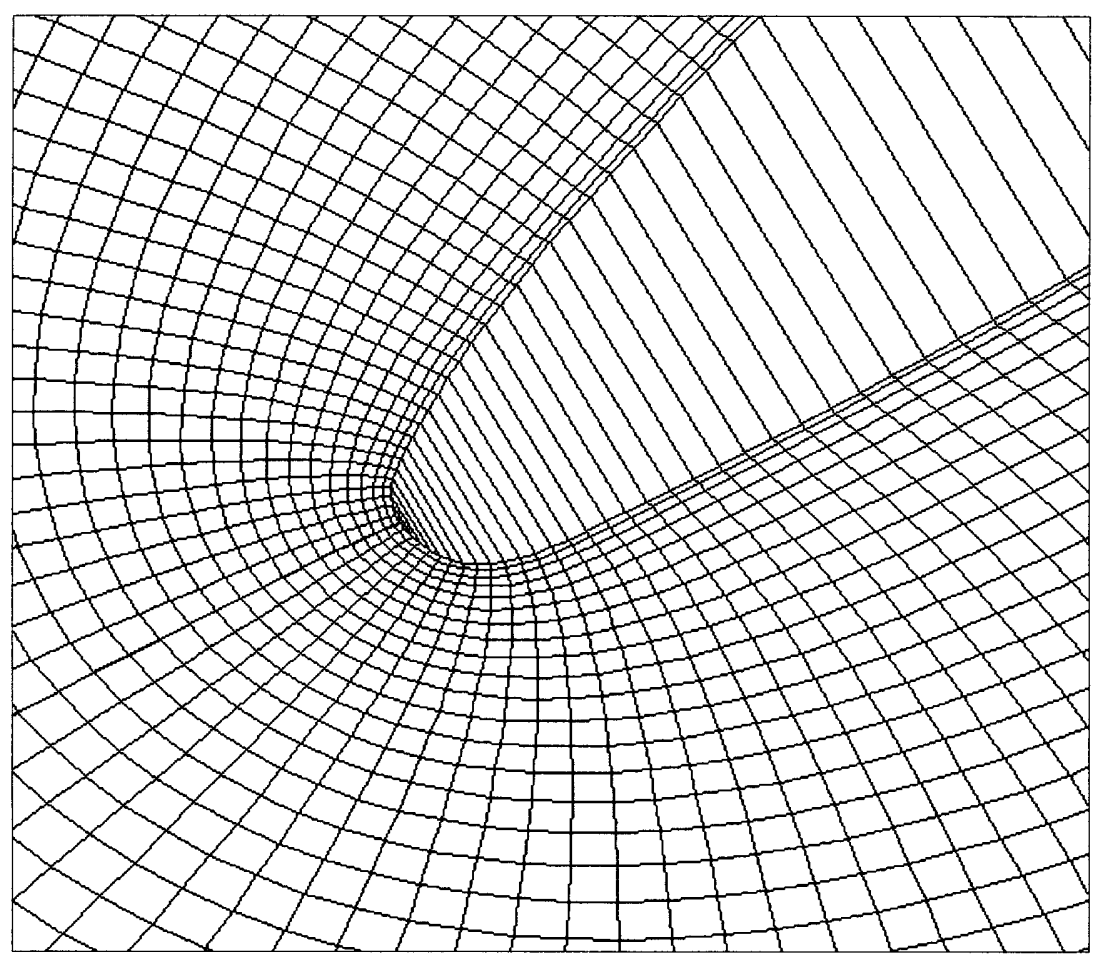

Figure 2-2: $\mathrm{O}-$ grid detail at the blade leading edge.

by Steger and Sorenson [20]. The O-grid detail near the blade leading edge and trailing edge is shown in Figure 2-2 and Figure 2-3. The grid cell aspect ratios obtained by this method are close to 1.0 near the blade leading and trailing edge. This is a typical "rule of thumb" value for grid cell aspect ratio in regions where the flow is not unidirectional [17]. The grid is also clustered closer at the blade surface and around the leading and trailing edge to help resolve the higher gradients in flow quantities in these regions. The Steger and Sorenson method also allows grid lines at the surface to be orthogonal to help simplify the evaluation of numerical derivatives. The grid is near-orthogonal in regions away from the surface.

The minimum grid resolution required to adequately capture flow features is a primary concern in any computational study. In the present study a detailed analysis of grid dependency was not conducted however. Instead the number of $\mathrm{H}$-grid and $\mathrm{O}$-grid nodes was increased until an upper limit was reached for the time required to achieve a converged solution (i.e. roughly equal to 3-4 weeks on a DEC Alpha workstation). The resulting Ogrid consists of 421 nodes around the blade surface and 32 nodes radially outward from the 


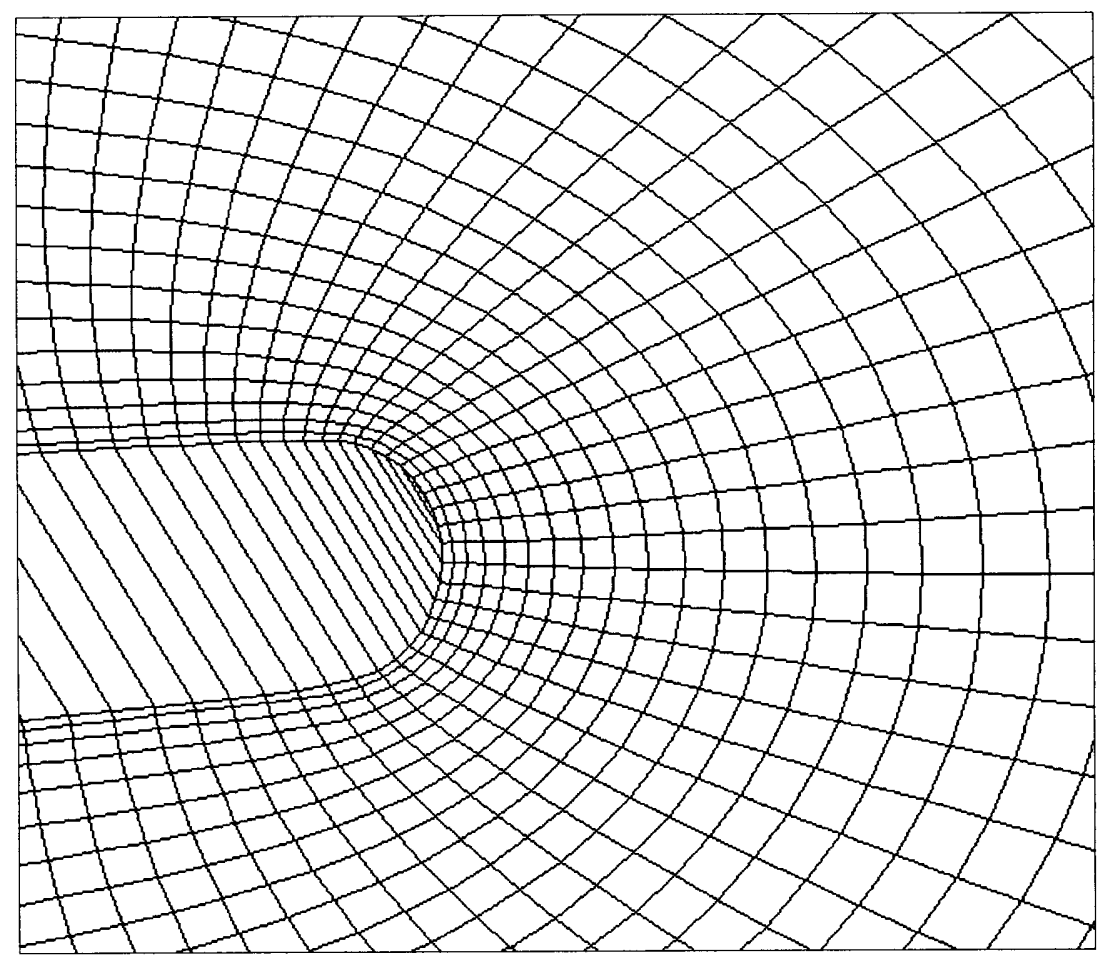

Figure 2-3: O-grid detail at the blade trailing edge.

blade surface. The H-grid consists of 161 nodes in the azimuthal direction and 1283 nodes in the axial direction.

\subsection{Cascade Geometry and Blade Profile}

The blade profile used in this study is the General Electric, Low Speed Research Compressor (LSRC) Stator-B blade. The 50\% span section of this blade shown in Figure 2-4 is used for all the viscous flow simulations. The LSRC blade design is described in detail by Wisler [26]. The blade and cascade geometry properties are listed in Table 2.1.

\subsection{Density Wake Profile}

A single density wake was convected through the cascade blade row in each simulation. The density wake, density variation is assumed to take a 1 -cosine functional form. This ensures 


\begin{tabular}{|l|l|}
\hline \hline Property & Value \\
\hline Space/chord ratio & 0.60 \\
Stagger angle & $24.5 \mathrm{deg}$. \\
Max. blade thickness & $0.071 c$ \\
Blade leading edge radius & $0.007 c$ \\
\hline \hline
\end{tabular}

Table 2.1: Properties of the LSRC cascade geometry used for the viscous CFD simulations.

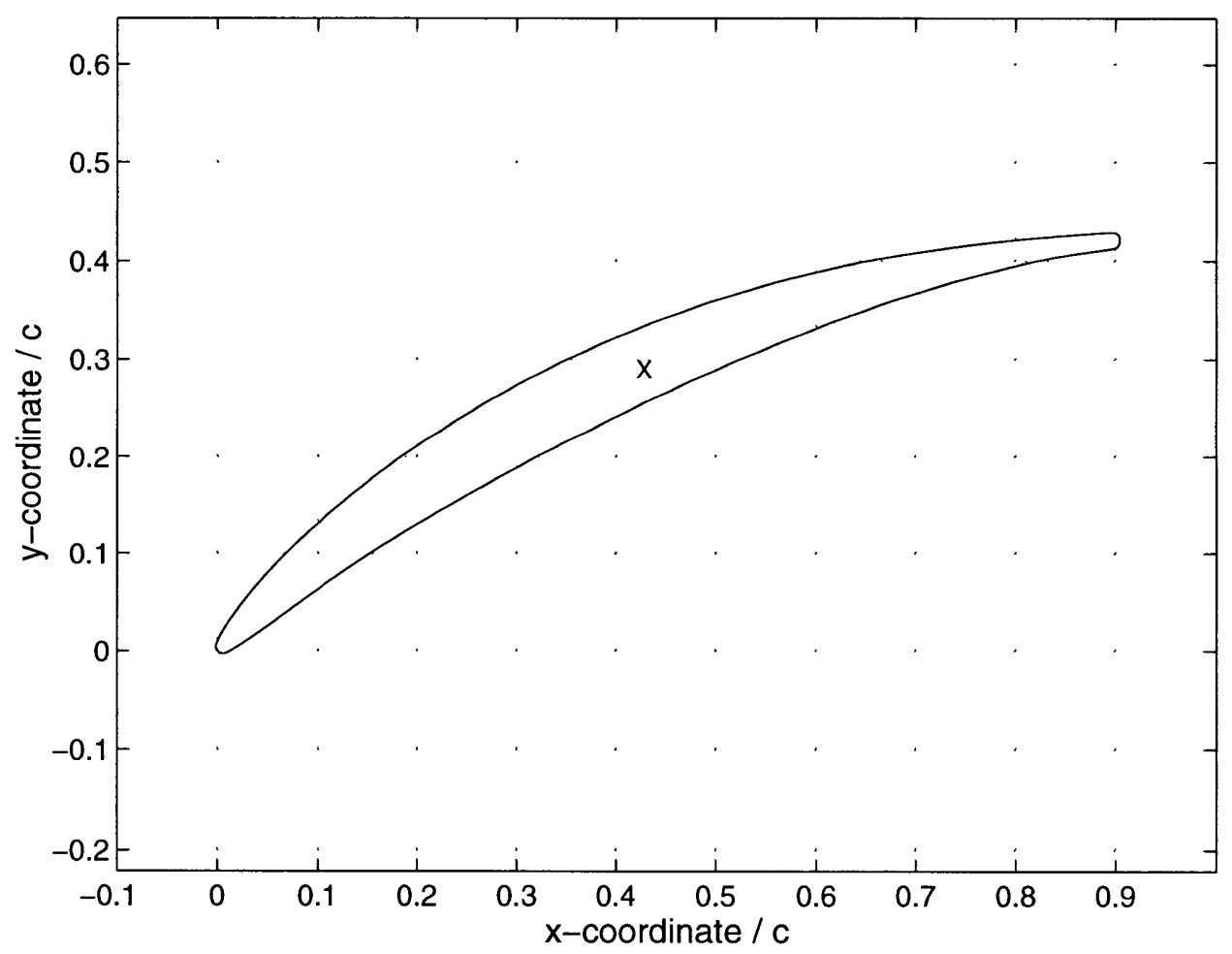

Figure 2-4: $50 \%$ span section of the LSRC Stator-B blade used for the viscous simulations. $\mathrm{X}$ denotes the location about which moments are evaluated $(0.42 \mathrm{c}, 0.29 \mathrm{c})$. 


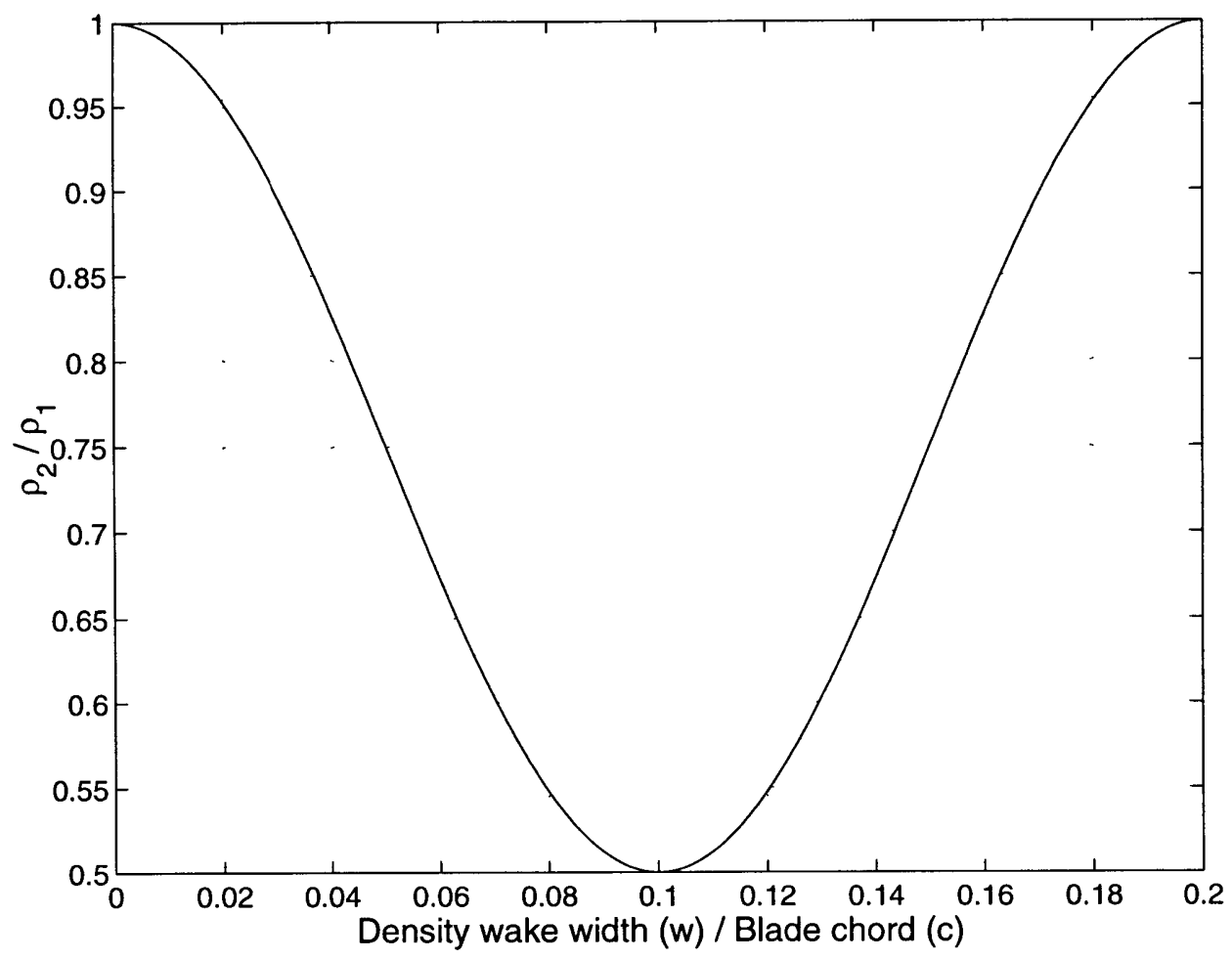

Figure 2-5: Density wake profile. $w / c=0.2, \rho_{2} / \rho_{1}=0.5$.

a smooth transition in density between the density wake and the free stream flow to help avoid possible numerical instabilities associated with step changes in flow properties. The density wake profile specified to represent a wake width $0.2 c$ and density ratio $\rho_{2} / \rho_{1}=0.5$ is illustrated in Figure 2-5.

\subsection{Converged Solutions}

The aim of the current research is to characterize the blade force and moment response induced by convecting density wakes. Before the density wakes can be introduced into the computational domain however a converged baseline solution must be obtained. The solution convergence was determined by monitoring the time history of the L2 norm of the flow variables $\rho, \rho u, \rho v$ and $\rho e$ where $\rho, u, v$, and $e$ denote density, axial velocity, azimuthal velocity and energy. A typical variation in the L2 norm for $\rho$ e is plotted in Figure 2-6. A converged solution was attained when the L2 norm for each flow variable showed no further significant reduction with time. In addition the flow field contour plots were examined to 


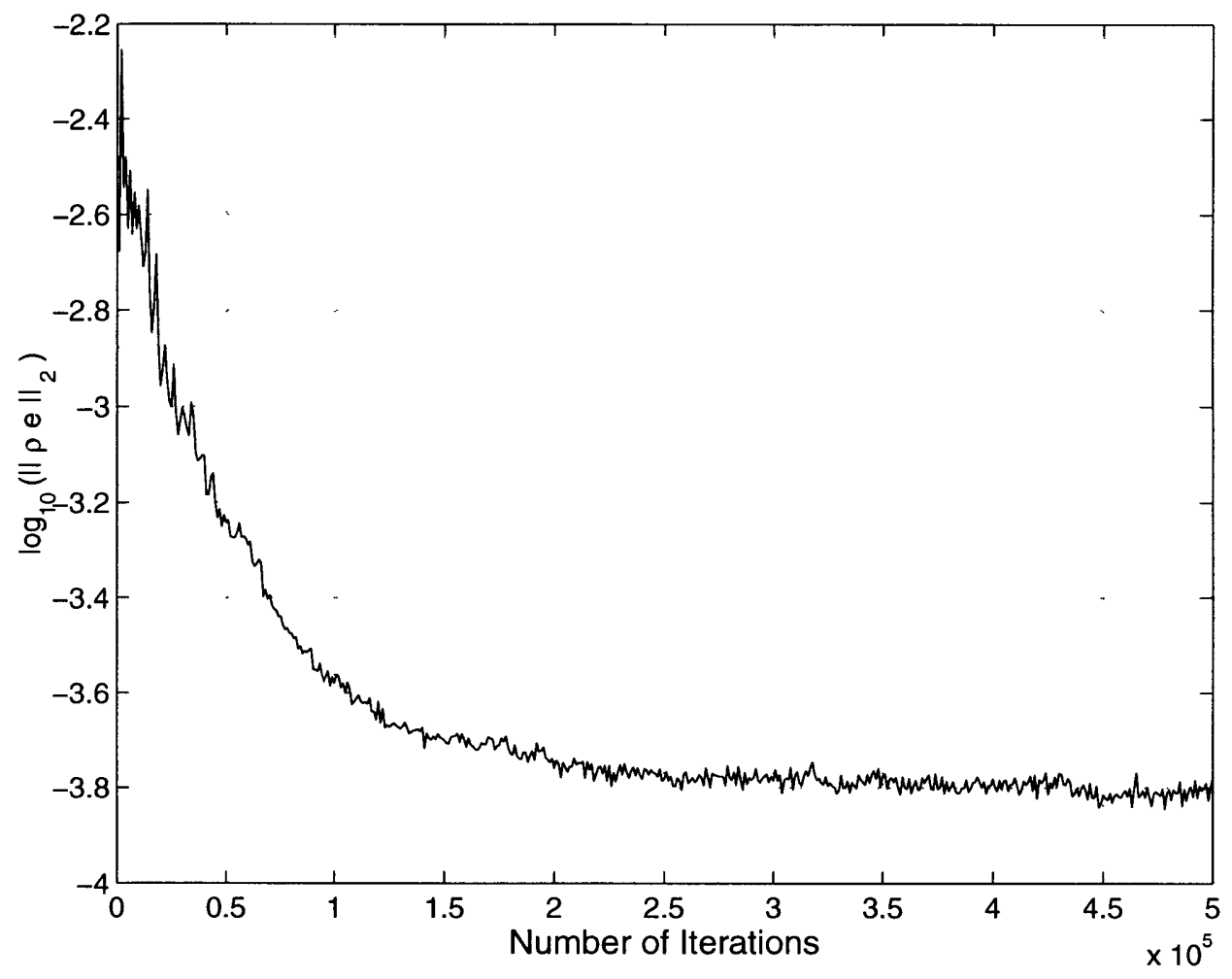

Figure 2-6: Convergence history for the L2 norm of density $\rho \times$ energy $e . M_{\infty}=0.53$.

ensure that unsteady transients were no longer present in the solution.

\subsection{Non-Dimensionalization}

The equations of motion are non-dimensionalized using the following reference quantities.

- Length : blade spacing $(s)$

- Velocity : rotor tip speed $\left(U_{R}\right)$

- Density : free stream density $\left(\rho_{\infty}\right)$

- Temperature : free stream temperature $\left(T_{\infty}\right)$

- Viscosity : free stream viscosity $\left(\mu_{\infty}\right)$

- Pressure : $\rho_{\infty} U_{R}^{2}$ 
- Energy : $U_{R}^{2}$

For the cascade geometry considered in this study the blade chord $c$ and inlet total velocity $U_{\infty}$ are more relevant non-dimensional variables for the length and velocity scales ${ }^{2}$. These variables are therefore used in the definitions for Reynolds number, Mach number, force coefficients, moment coefficient (positive clockwise about $x / c=0.42, y / c=0.29$ ) and skin friction coefficient as follows,

$$
\begin{aligned}
R e_{\infty} & =\frac{\rho_{\infty} U_{\infty} c}{\mu_{\infty}} \\
M_{\infty} & =\frac{U_{\infty}}{\sqrt{\gamma R T_{\infty}}} \\
C y & =\frac{p \Delta x}{0.5 \rho_{\infty} U_{\infty}^{2} c} \\
C x & =\frac{p \Delta y}{0.5 \rho_{\infty} U_{\infty}^{2} c} \\
C m & =\frac{p \Delta y \Delta l}{0.5 \rho_{\infty} U_{\infty}^{2} c^{2}} \\
C f & =\frac{\tau_{w}}{0.5 \rho_{\infty} U_{\infty}^{2}}
\end{aligned}
$$

Note the flow Reynolds number and Mach number based on the inlet total velocity and blade chord are determined after a converged solution is obtained and cannot be specified a-priori. The Reynolds numbers and Mach numbers required as input conditions to the flow solver are based on the blade spacing and rotor tip speed instead ${ }^{3}$.

\footnotetext{
${ }^{2} \mathrm{An}$ additional factor of 0.5 for the pressure non-dimensionalization is also more appropriate

${ }^{3}$ Since there is no rotor in this study the values for Reynolds number and Mach number specified as input conditions are essentially unit values.
} 


\subsection{Summary}

The key features of the computational scheme used for the viscous flow simulations have been described in this Chapter. The following 3 features have helped to reduce the computational cost associated with this scheme:

1. High order scheme with fourth-order spatial accuracy and third-order temporal accuracy. The added cost to compute the derivatives is offset by the savings in the number of required grid points.

2. The Navier-Stokes equations are solved only within an $\mathrm{O}$-grid region surrounding the blade surfaces. The less expensive Euler equations are solved in the remaining flow regions upstream and downstream away from the blade surfaces.

3. Wall functions are used to model the inner region of the blade boundary layer. This dramatically reduces the required number of near wall grid points.

The convergence of the baseline flow solutions are determined by tracking the change in the L2 norm of the conserved flow variables. A detailed grid dependency study was not conducted in this research. Instead the number of grid points were increased until an upper limit was reached for the time required to achieve a converged solution. A typical converged flow solution requires 3-4 weeks on a DEC Alpha workstation. 


\section{Chapter 3}

\section{Viscous Results: Baseline SOLUTIONS}

The aim of this Chapter is to characterize the vortex shedding induced force and moment fluctuations for the baseline flow solutions before density wakes are introduced. The amplitude of the force and moment fluctuations in the baseline flow are significant and indicate that vortex shedding is a possible additional source for high cycle fatigue failure.

\subsection{Baseline Force and Moment Response}

Table 3.1 summarizes the flow field properties for all the viscous compressible baseline flows considered in this research. The time history of the blade force and moment fluctuations for each converged baseline solution is plotted in Figure A-5 through Figure A-8 in Appendix A. The time averaged value of these coefficients and the amplitude of the fluctuations are tabulated in Table 3.2. While the azimuthal force coefficient shows the largest amplitude fluctuations (for a given Mach number) the moment coefficient fluctuations show the largest percentage fluctuation from the time averaged mean value.

Vortex shedding results in a change in circulation around the cascade blades such that the net circulation of the cascade blades and shed vortices remains constant (by Kelvin's theorem). This is strictly true for inviscid flows but is a good approximation when viscous effects are confined to thin shear layers [4]. The change in the blade circulation results in a 


\begin{tabular}{|c|c|c|c|c|}
\hline \hline Flow Property & Run 1 & Run 2 & Run 3 & Run 4 \\
\hline$M\left(U_{T}\right)$ (specified) & 0.20 & 0.70 & 0.85 & 0.90 \\
$M\left(U_{\infty}\right)$ (computed) & 0.15 & 0.53 & 0.63 & 0.87 \\
$\operatorname{Re}\left(U_{T}, s\right)$ (specified) & 500,000 & 500,000 & 500,000 & 500,000 \\
$\operatorname{Re}\left(U_{\infty}, c\right)$ (computed) & 620,000 & 630,000 & 580,000 & 800,000 \\
$\Psi_{s-s}$ (computed) & 0.46 & 0.46 & 0.45 & 0.47 \\
\hline \hline
\end{tabular}

Table 3.1: Viscous flow simulations. The Reynolds number and Mach number specified in the solver are based on unit blade spacing and unit velocity.

\begin{tabular}{|c|c|c|c|}
\hline \hline Inlet Mach No. & $C y_{\text {mean }}$ & $C x_{\text {mean }}$ & $C m_{\text {mean }}$ \\
\hline Run 1 $\left(M_{\infty}=0.15\right)$ & $0.44 \pm 0.0044$ & $0.23 \pm 0.0023$ & $0.08 \pm 0.0016$ \\
Run 2 $\left(M_{\infty}=0.53\right)$ & $0.38 \pm 0.0114$ & $0.24 \pm 0.0048$ & $0.07 \pm 0.0063$ \\
Run 3 $\left(M_{\infty}=0.63\right)$ & $0.36 \pm 0.0144$ & $0.23 \pm 0.0046$ & $0.06 \pm 0.0054$ \\
Run 4 $\left(M_{\infty}=0.87\right)$ & $0.33 \pm 0.0160$ & $0.21 \pm 0.0063$ & $0.06 \pm 0.0078$ \\
\hline \hline
\end{tabular}

Table 3.2: The time averaged force and moment coefficients for the baseline solutions ranging from $M_{\infty}=0.15$ to $M_{\infty}=0.87$.

change in the blade pressure distribution and therefore a corresponding fluctuation in the blade force and moment coefficients.

The fluctuation in force and moment coefficients for each Mach number flow is examined separately in the following sections. A combination of (1) flow field vorticity contour images, (2) vortex trajectory plots and (3) fourier transforms of the force and moment fluctuations are used to highlight the flow features.

\subsubsection{Run 1: $\mathrm{M}_{\infty}=0.15$}

The computed vorticity contours in Figure 3-1 clearly indicates the periodic vortex shedding at the blade trailing edge for Run 1. The shear layers from the blade pressure and suction surface are seen to leave the blade trailing edge smoothly and undergo transverse oscillations normal to the flow direction. These oscillations gradually increase in amplitude and eventually cause roll up of the shear layers at a distance $0.3 c$ downstream of the trailing edge. A Karman-Vortex street is then formed with alternating clockwise and counterclock- 
wise vortices arranged in a " $2 \mathrm{~S}$ " type configuration [25].

The frequency of the shear layer oscillations can be determined by tracking the location of consecutive wake peaks as shown in Figure 3-2. The reduced frequency of the shear layer oscillations determined from this plot is $\mu=4.0$. The reduced frequency for the formation of same-sign vortices further downstream can be obtained in a similar manner and is found to occur at $\mu=3.5$. The formation of alternate-sign vortices occurs at twice this frequency.

These shear layer oscillation frequencies and vortex formation frequencies appear as relatively small peaks in the frequency spectrum for the Run 1 force and moment fluctuations shown in Figure 3-3. Higher frequencies at $\mu=9.5,22.0,34.5$ and 43.5 are instead found to dominate the response. The $\mu=9.5$ frequency peak is found to correspond with static pressure fluctuations at the inlet and exit boundaries. The source of all other frequencies could not be precisely determined however. It is possible that these frequencies originate from numerical discretization errors and/or undamped acoustic modes. The close proximity of the inlet and exit boundaries to the blade profile may also be a factor ${ }^{1}$. The net contribution of these higher frequencies to the amplitude of the force and moment fluctuations is nevertheless bounded and less than $2 \%$.

\subsubsection{Run 2: $\mathrm{M}_{\infty}=0.53$}

The vortex shedding behind the blade trailing edge for Run 2 is plotted in Figure 3-4. Individual vortices are seen here to form immediately behind the blade trailing edge. The pressure and suction surface shear layers do not extend downstream of the blade and no gradual growth in shear layer oscillations can be observed.

The trajectory of consecutive same-sign vortices in Run 2 are plotted in Figure 3-5. The reduced frequency for vortex shedding determined from this plot is $\mu=3.0$.

\footnotetext{
${ }^{1}$ An additional low subsonic simulation on an extended computational domain is required to resolve this issue.
} 


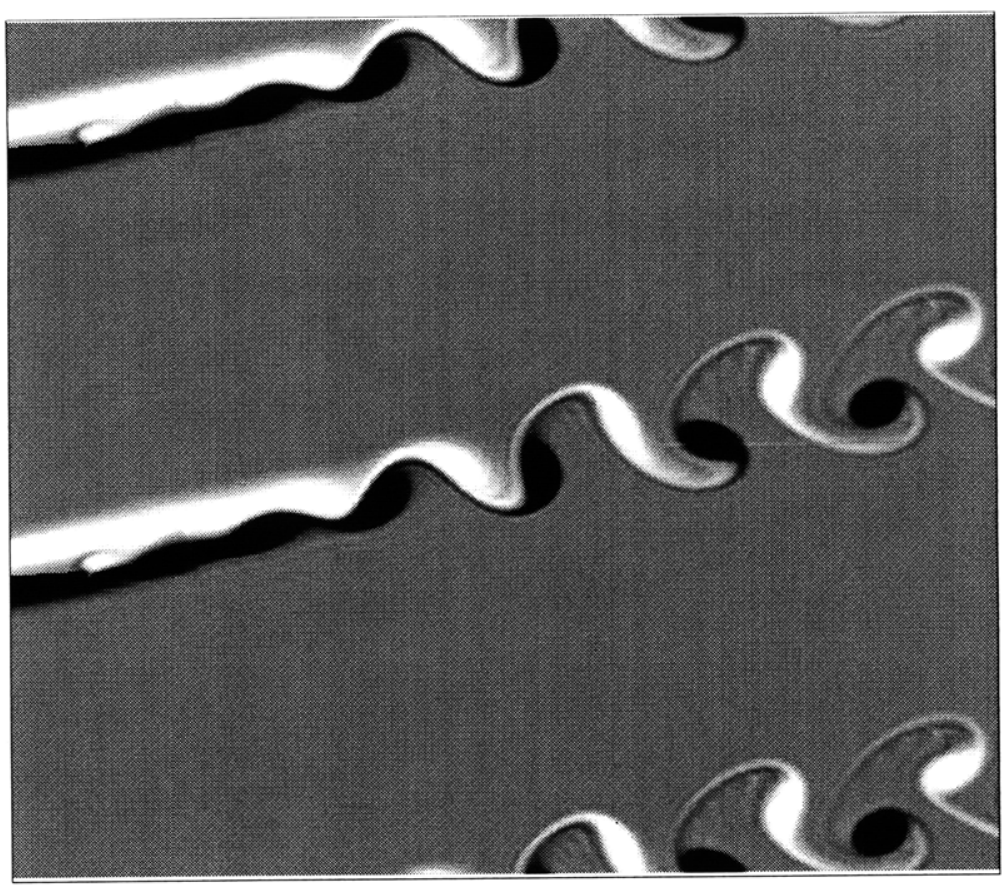

Figure 3-1: Vorticity contours indicating vortex shedding behind the blade trailing edge. $M_{\infty}=$ 0.15. $\operatorname{Re}\left(c, U_{\infty}\right)=620,000$. The blade trailing edge is located at $\approx 0.40 \times$ (spacing of same-sign vortices) to the right of the left boundary.

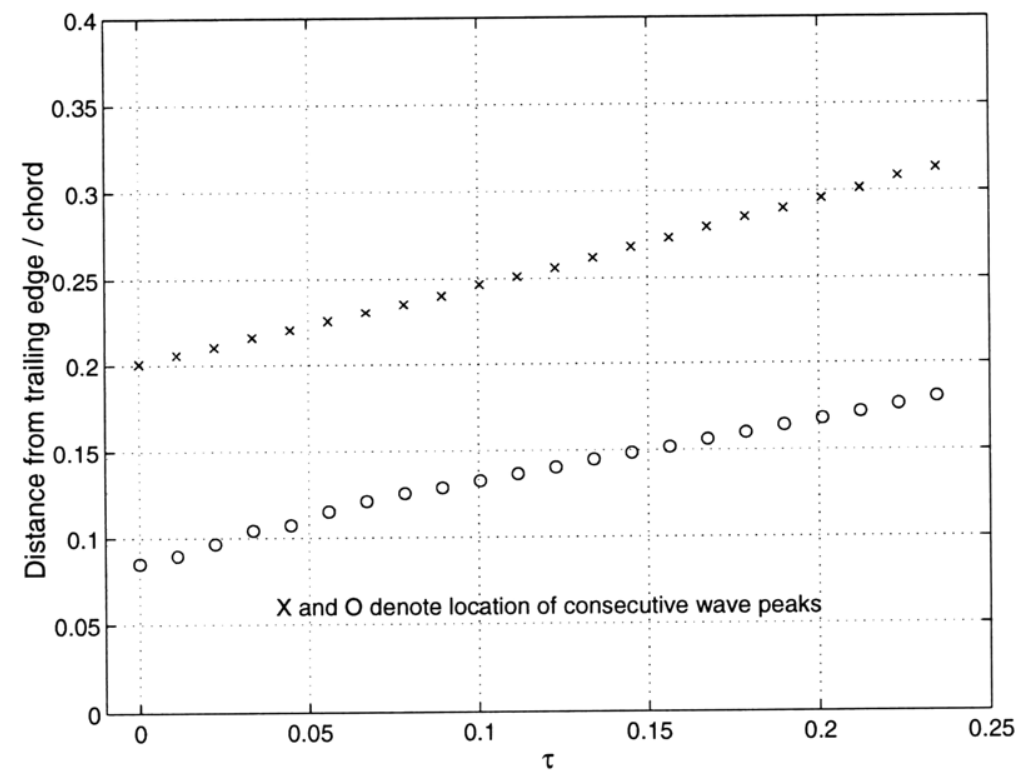

Figure 3-2: Trajectory of consecutive shear layer wave peaks at an arbitrary time instance. $M_{\infty}=0.15$. 

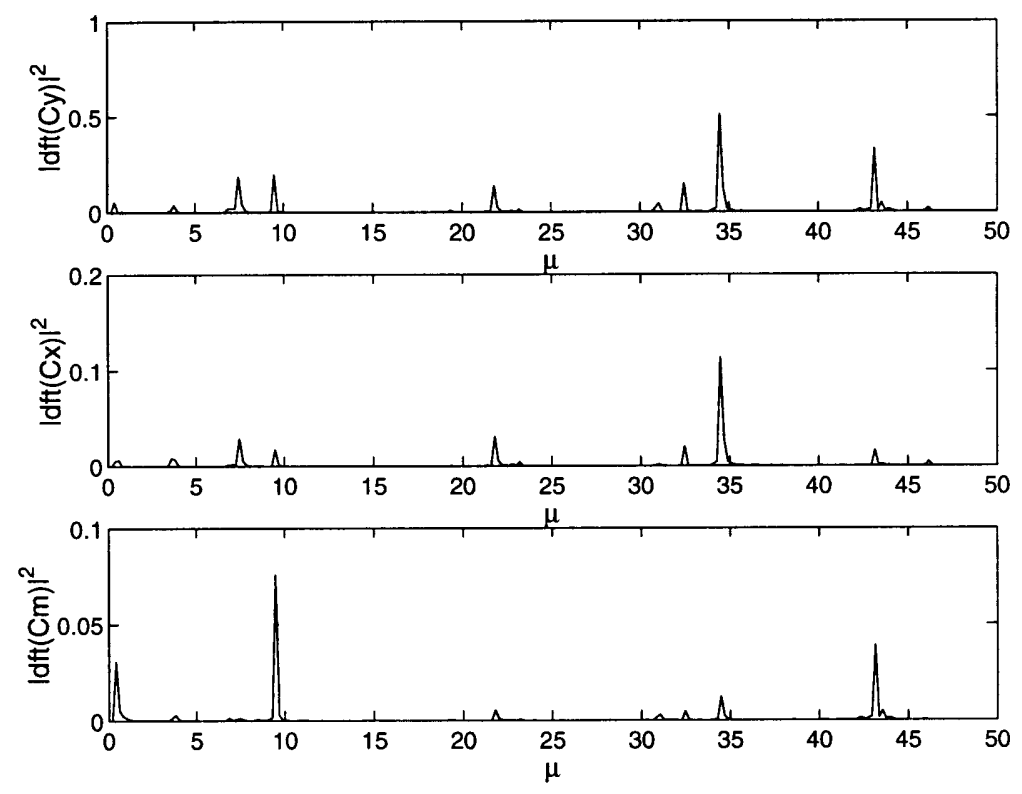

Figure 3-3: Discrete fourier transform of the baseline force and moment coefficients. $d f t(X)$ is the discrete fourier transform of the time signal $X . \mu=$ non-dimensional frequency. $M_{\infty}=0.15$.

The frequency spectrum of the Run 2 force and moment fluctuations is plotted in Figure 3-6. The vortex shedding frequency at $\mu=3.0$ is dominant in the frequency response. The peak at $\mu=6.0$ corresponds to the frequency of alternate-sign vortex shedding. Note that the $\mu=6.0$ frequency peak is not captured in the moment coefficient fourier transform. This is contrary to the moment coefficient fluctuations observed for circular cylinders which occur at twice the vortex shedding frequency. The shear layers which separate from cascade blades are closer together however and the vortex shedding from both the suction and pressure surfaces appears to add to the moment coefficient fluctuation with equal phase. This also explains the overall larger amplitude of the moment coefficient fluctuations.

The reason for the increased correlation between the vortex shedding frequency and the force and moment fluctuation frequencies in Run 2 compared to Run 1 cannot be precisely concluded upon. The closer proximity of vortex shedding in Run 2 to the blade trailing edge is however considered to be a key factor. The shed vortices in Run 2 therefore exert a greater influence on the blade pressure distribution compared to the vortices in Run 1 which are further downstream. This would also explain the overall larger force and moment 


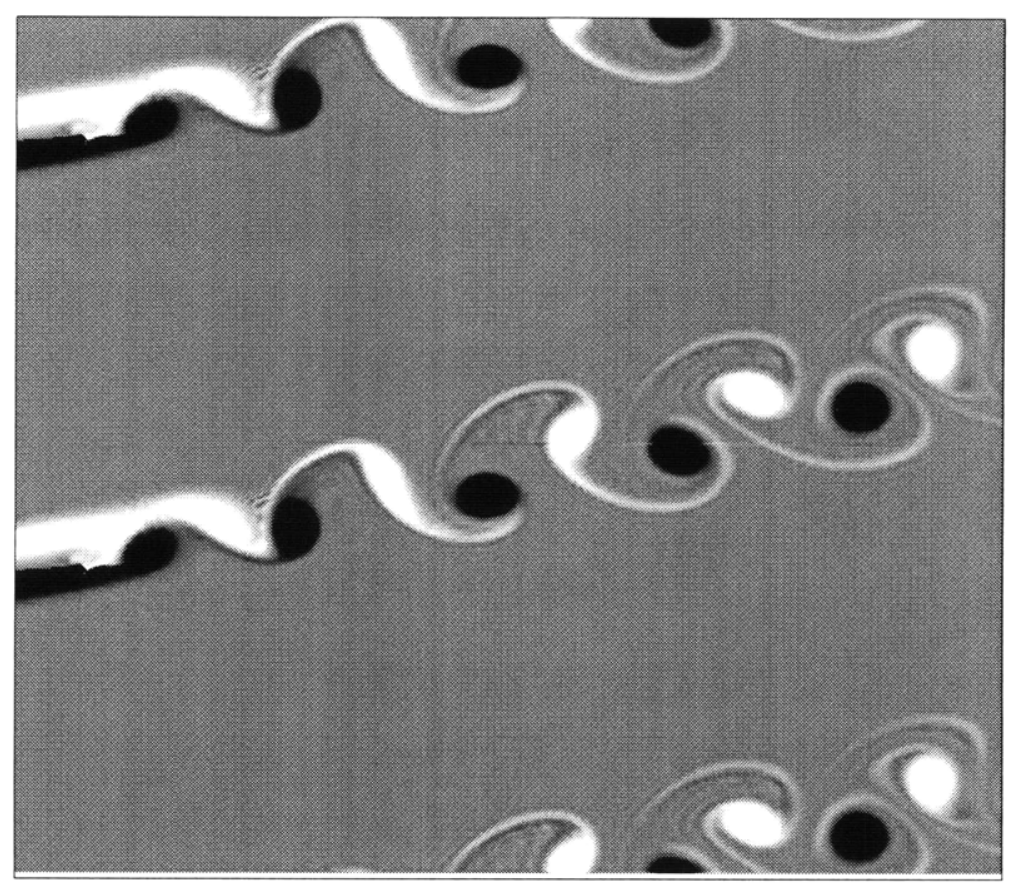

Figure 3-4: Vorticity contours indicating vortex shedding behind the blade trailing edge. $M_{\infty}=$ 0.53. $\operatorname{Re}\left(c, U_{\infty}\right)=630,000$. The blade trailing edge is located at $\approx 0.35 \times$ (spacing of same-sign vortices) to the right of the left boundary.

fluctuation amplitudes in Run 2 compared to Run 1 (see Table 3.2).

\subsubsection{Run 3: $\mathrm{M}_{\infty}=0.63$}

The vortex shedding pattern behind the blade trailing edge for Run 3 is shown in Figure 3-7. Similar to Run 2, vortex shedding occurs immediately behind the blade trailing edge.

The trajectory of a typical same-sign vortex pair in Run 3 is plotted in Figure 3-7. The reduced frequency for vortex shedding calculated from this trajectory is $\mu=2.9$.

The frequency spectrum for the Run 3 force and moment fluctuations is plotted in Figure 3-9. The vortex shedding frequency at $\mu=2.9$ is dominant in this response. There is no major differences between the Run 2 and Run 3 baseline solutions. The amplitude of the force and moment coefficient fluctuations are almost identical (see Table 3.2). This is not surprising considering the difference in free stream Mach numbers between the two 


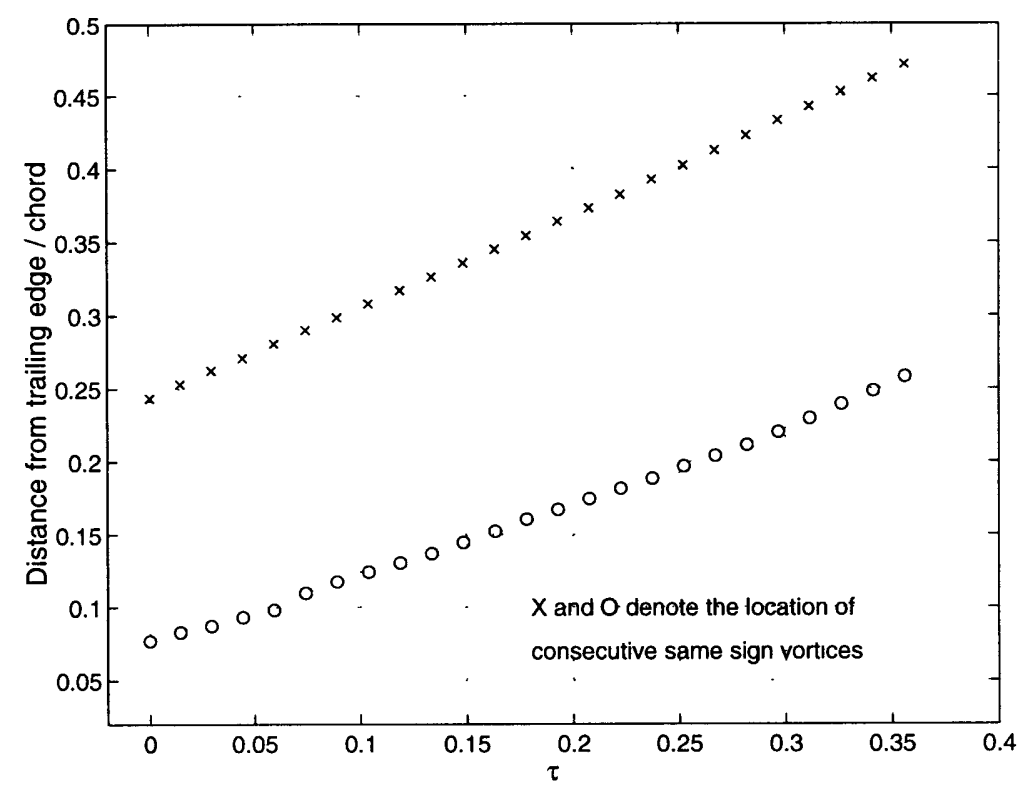

Figure 3-5: Trajectory of consecutive same sign vortices at an arbitrary time instance. $M_{\infty}=$ 0.53 .
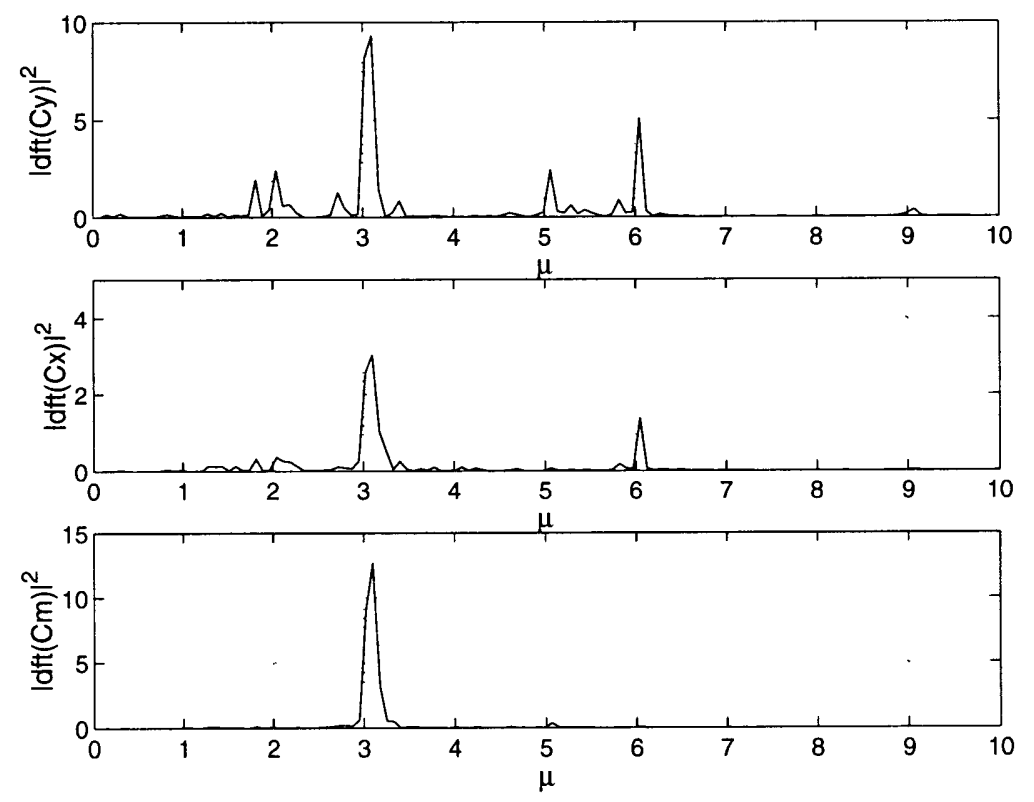

Figure 3-6: Discrete fourier transform of the baseline force and moment coefficients. $d f t(X)$ is the discrete fourier transform of the time signal $X . \mu=$ non-dimensional frequency. $M_{\infty}=0.53$. 


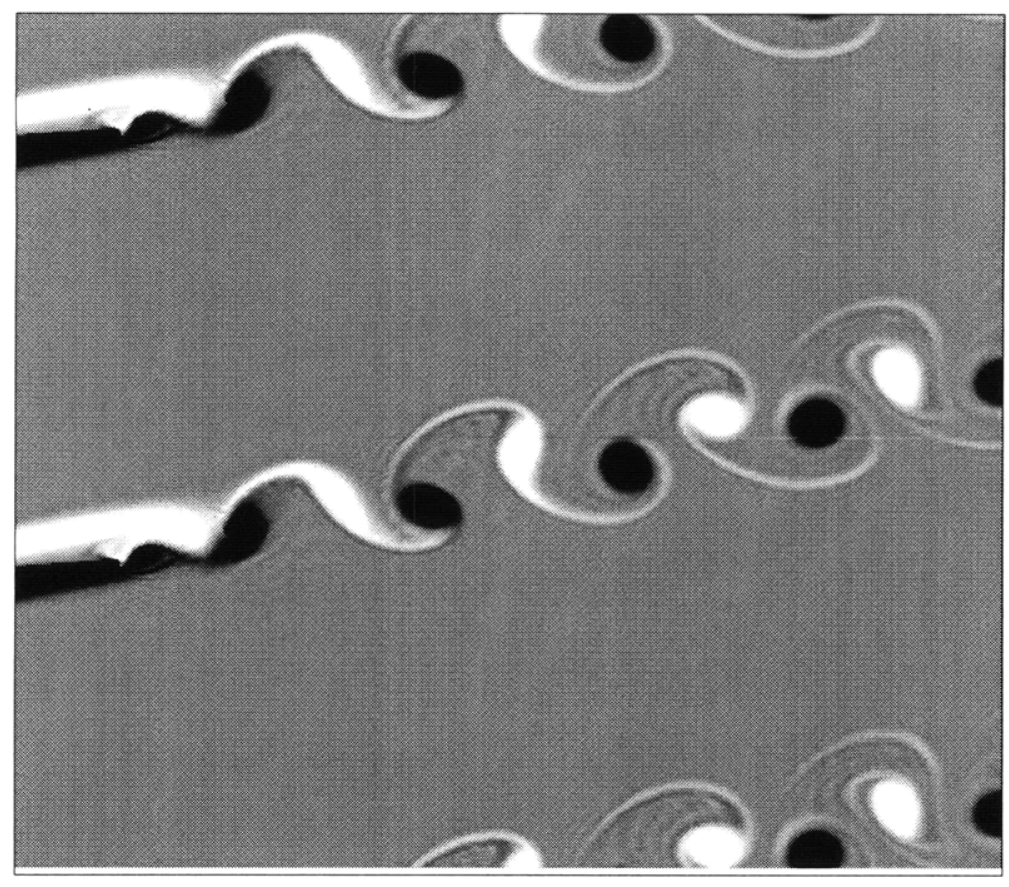

Figure 3-7: Vorticity contours indicating vortex shedding behind the blade trailing edge. $M_{\infty}=$ 0.63. $\operatorname{Re}\left(c, U_{\infty}\right)=580,000$. The blade trailing edge is located at $\approx 0.50 \times$ (spacing of same-sign vortices) to the right of the left boundary.

tests is only 0.1 .

\subsubsection{Run 4: $\mathrm{M}_{\infty}=0.87$}

The baseline solution for Run 4 consists of a shock wave located a distance $0.25 c$ downstream of the blade leading edge. This shock wave can be deduced from the Mach number contour plot in Figure 3-10. The region of supersonic flow begins $0.06 \mathrm{c}$ aft of the blade leading edge and extends roughly halfway across the blade passage. The space-averaged flow properties across the shock wave are compared with one-dimensional normal shock properties in Table 3.3. The pressure ratio, temperature ratio and density ratio are seen to compare to within $1 \%$.

Vortex shedding in Run 4 is similar to Run 2 and Run 3 with vortices formed immediately behind the blade trailing edge as shown in Figure 3-11. The trajectory of two consecutive same-sign vortices are plotted in Figure 3-12. This trajectory indicates the reduced frequency for vortex shedding to be $\mu=1.9$. The frequency spectrum of the Run 4 


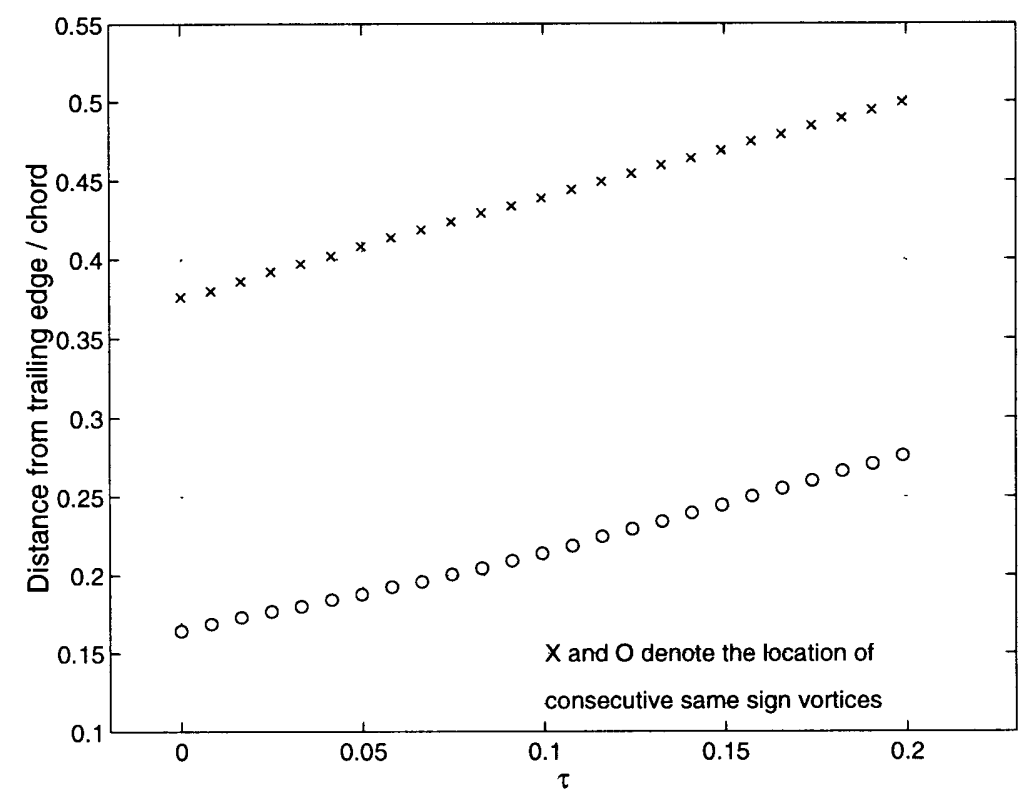

Figure 3-8: Trajectory of consecutive same-sign vortices at an arbitrary time instance. $M_{\infty}=$ 0.63 .
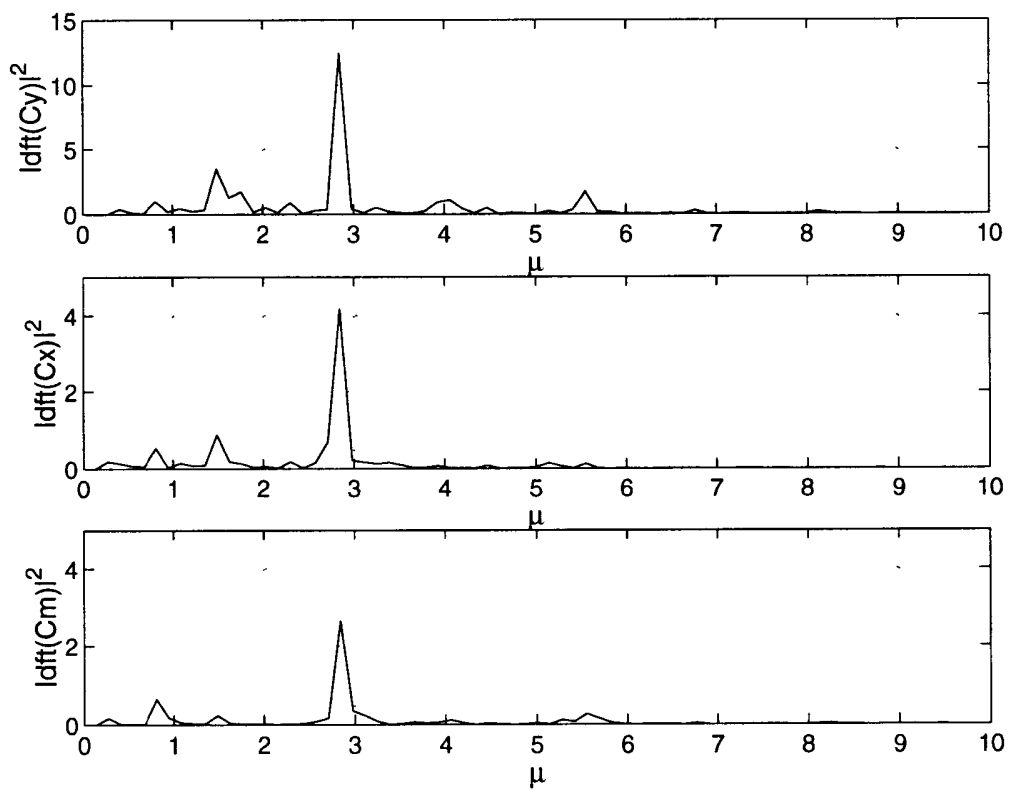

Figure 3-9: Discrete fourier transform of the baseline force and moment coefficients. $d f t(X)$ is the discrete fourier transform of the time signal $X . \mu=$ non-dimensional frequency. $M_{\infty}=0.63$. 


\begin{tabular}{|c|c|c|}
\hline \hline Property & Viscous CFD & 1D Normal Shock \\
\hline$M_{1}$ & 1.29 & 1.29 \\
$M_{2}$ & 0.81 & 0.79 \\
$p_{2} / p_{1}$ & 1.76 & 1.77 \\
$\rho_{2} / \rho_{1}$ & 1.49 & 1.50 \\
$T_{2} / T_{1}$ & 1.18 & 1.18 \\
\hline \hline
\end{tabular}

Table 3.3: Comparison of Run 4 shock wave properties with $1 \mathrm{D}$ normal shock properties. $\gamma=1.4$.

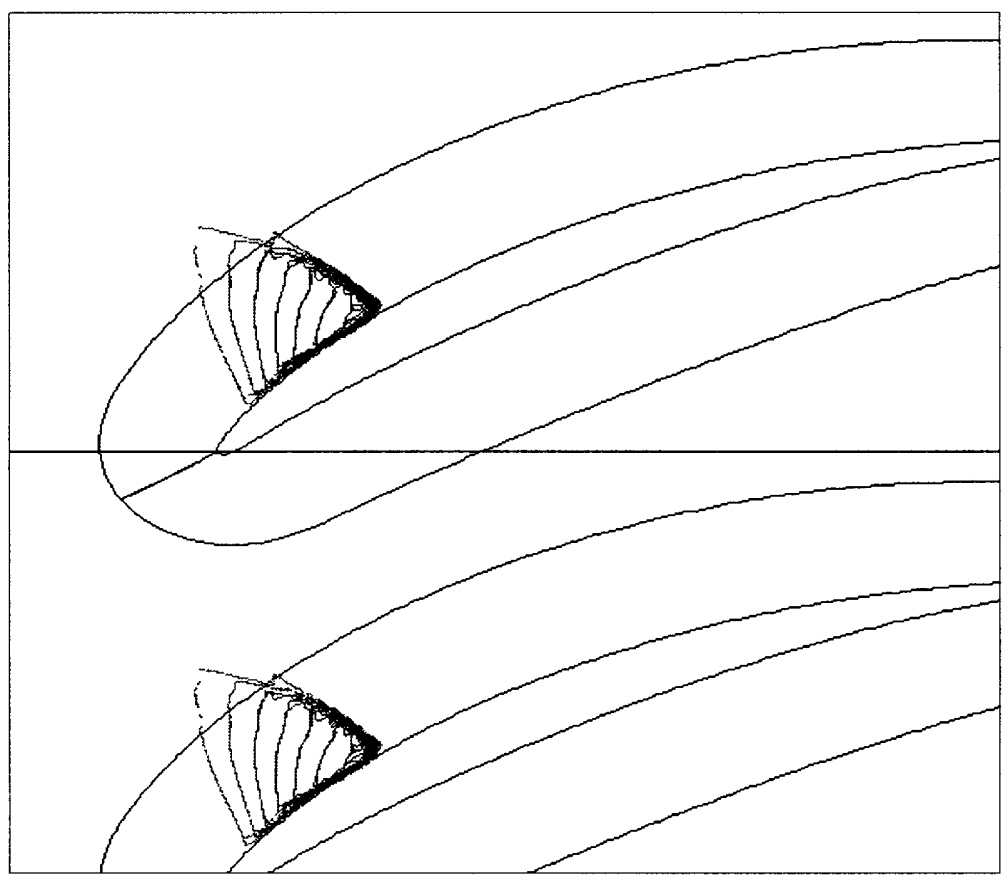

Figure 3-10: Mach number contours indicating extent of supersonic region in Run 4. Contours range from $M=1.0$ to $M=1.5$ in steps of 0.05 . $M_{\infty}=0.87$.

force and moment fluctuations in Figure 3-13 show good agreement with peaks at $\mu=2.0$ for both the azimuthal force coefficient and the moment coefficient. The frequency spectrum for the axial force coefficient however shows a smaller peak at $\mu=2.0$ and instead has a broader distribution of frequencies ${ }^{2}$. Furthermore the amplitude of the coefficient fluctuations for Run 4 are greater than the fluctuation amplitudes observed at all other Mach numbers.

The contribution to this greater amplitude and broader spectrum of frequencies orig-

\footnotetext{
${ }^{2}$ The azimuthal force coefficient and the moment coefficient also show the same frequency peaks however with smaller amplitude.
} 


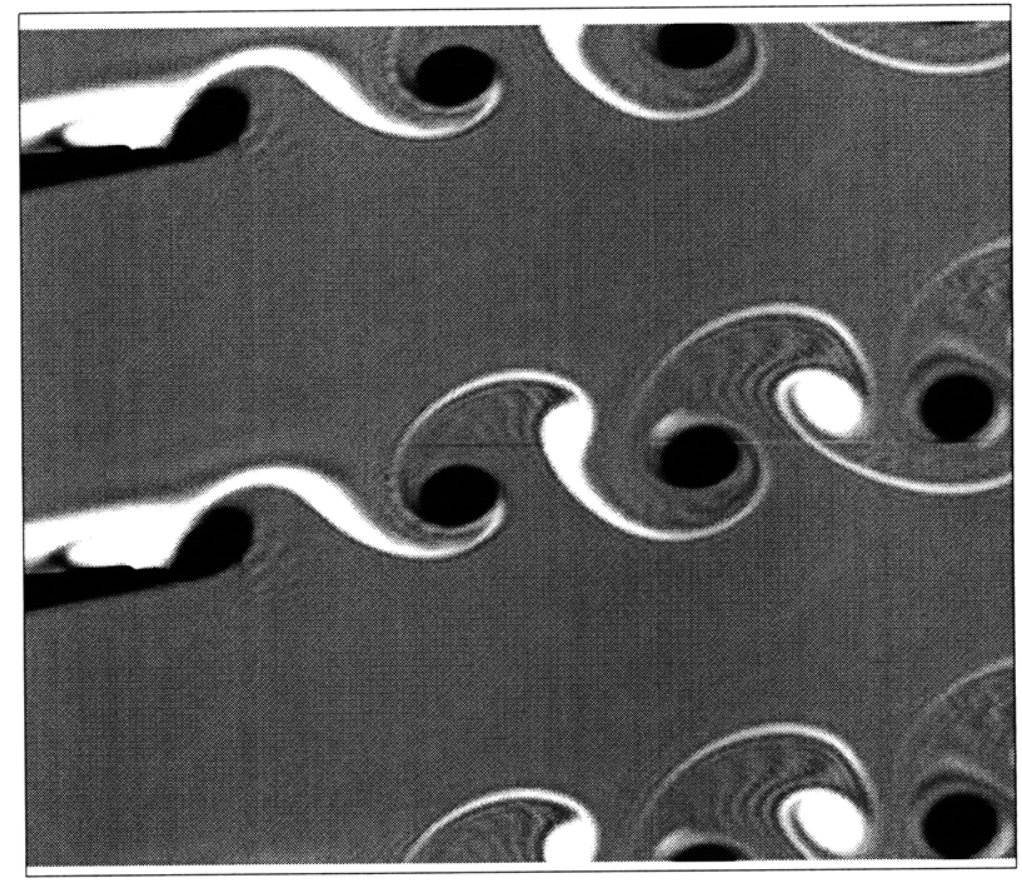

Figure 3-11: Vorticity contour images indicating vortex shedding behind the blade trailing edge. $M_{\infty}=0.87 . \operatorname{Re}\left(c, U_{\infty}\right)=800,000$. The blade trailing edge is located at $\approx 0.50 \times$ (spacing of same-sign vortices) to the right of the left boundary.

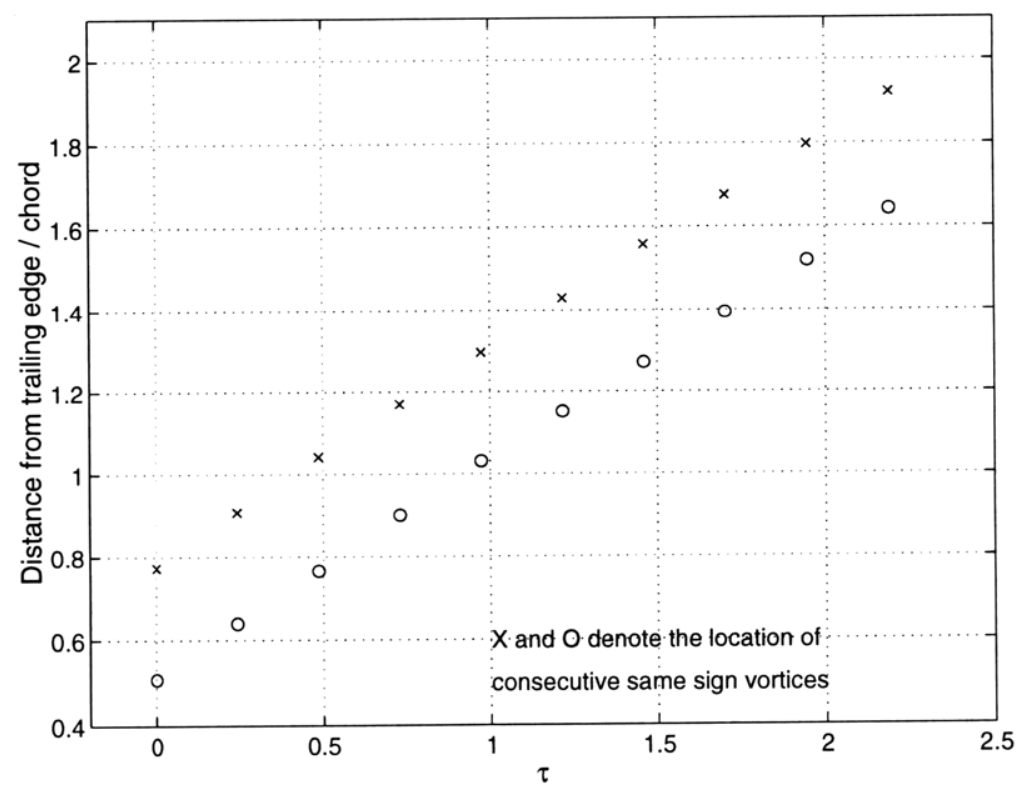

Figure 3-12: Trajectory of consecutive same sign vortices at an arbitrary time instance. $M_{\infty}=$ 0.87 . 

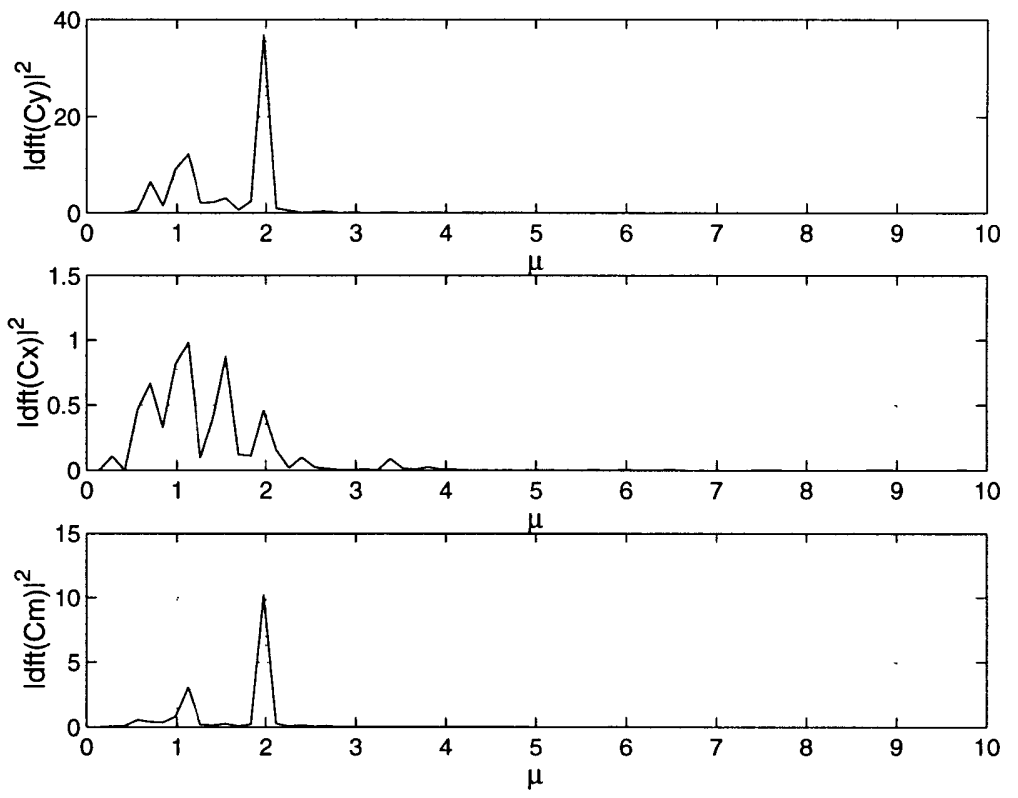

Figure 3-13: Discrete fourier transform of the baseline force and moment coefficients. $d f t(X)$ is the discrete fourier transform of the time signal X. $\mu=$ non-dimensional frequency. $M_{\infty}=0.87$.

inates from upstream traveling pressure waves generated by vortex shedding at the blade trailing edge. These pressure waves can be seen in Figure 3-14 as dark bands which extend across the blade passage. Similar vortex shedding induced pressure waves have been observed by Lawaczeck and Heinemann [14] and Heinemann and Butefisch [8] in the flow past flat plate cascades and turbine cascades at transonic Mach numbers and Reynolds numbers of 0.8 million. A schlieren image taken from Lawaczeck shown in Figure 3-15 clearly indicates these pressure waves. Lawaczeck notes that each shed vortex generates a pressure pulse at the trailing edge.

The flow properties at 3 azimuthal locations across the blade passage were obtained to determine the theoretical propagation speed $(U-a)$ of a typical pressure wave. Table 3.4 lists the flow properties at the 3 azimuthal locations. An average propagation velocity $(U-a)$ of $0.27 U_{\infty}$ is obtained. This value can now be compared with the velocity obtained by tracking the propagation of the pressure wave fronts shown schematically in Figure 3-16. A velocity of $0.28 U_{\infty}$ is obtained which is within $4 \%$ of the theoretical value.

As the pressure waves intercept the shock wave they are seen to displace the shock 


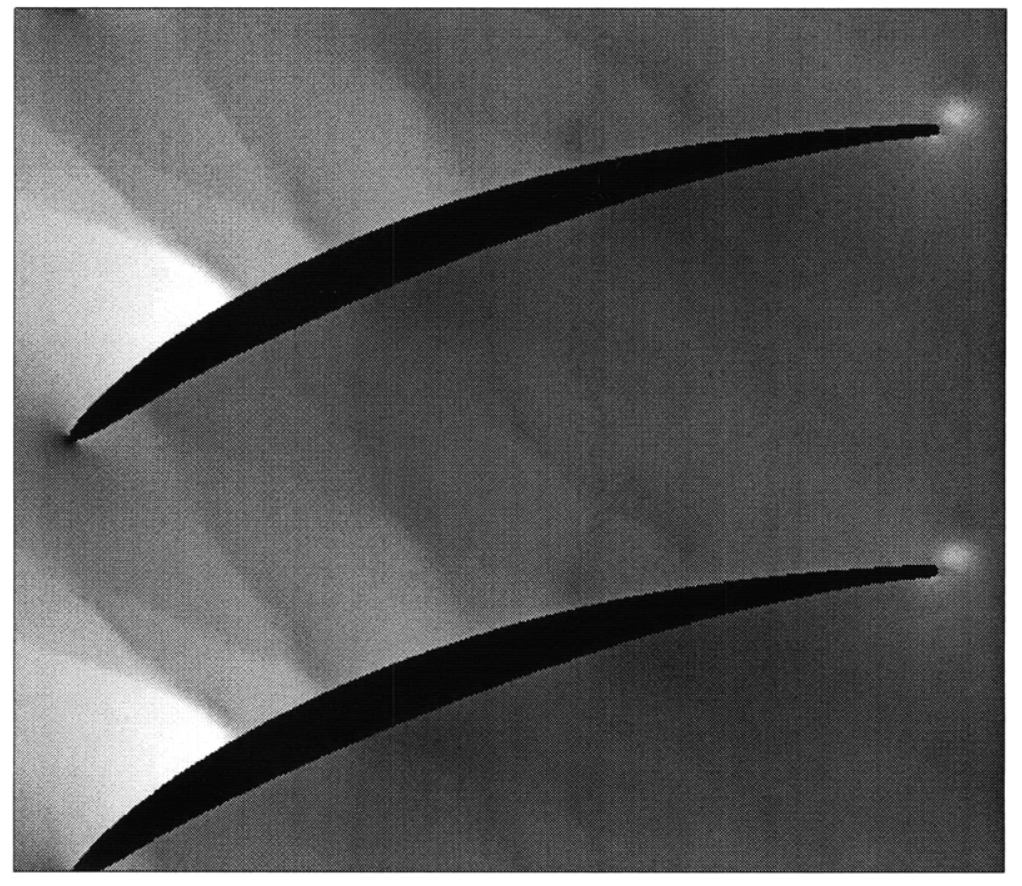

Figure 3-14: Pressure contours of the baseline solution for Run 4. The pressure waves can be seen as dark lines extending across the blade passage. $M_{\infty}=0.87 . \operatorname{Re}\left(c, U_{\infty}\right)=$ $0.8 \times 10^{6}$.

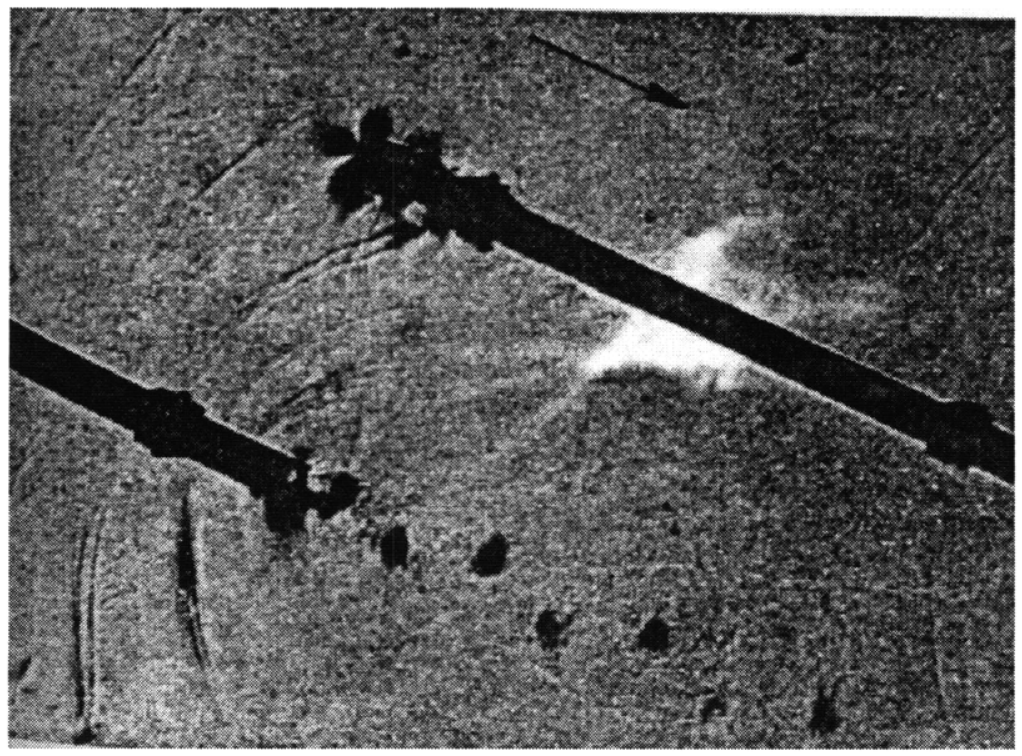

Figure 3-15: Schlieren image of upstream traveling pressure waves behind a flat plate cascade from Lawaczeck. The Flow is from left to right. $M$ (trailing edge) $\approx 0.80$. $\operatorname{Re}\left(c, U_{\infty}\right)=0.8 \times 10^{6}$. 


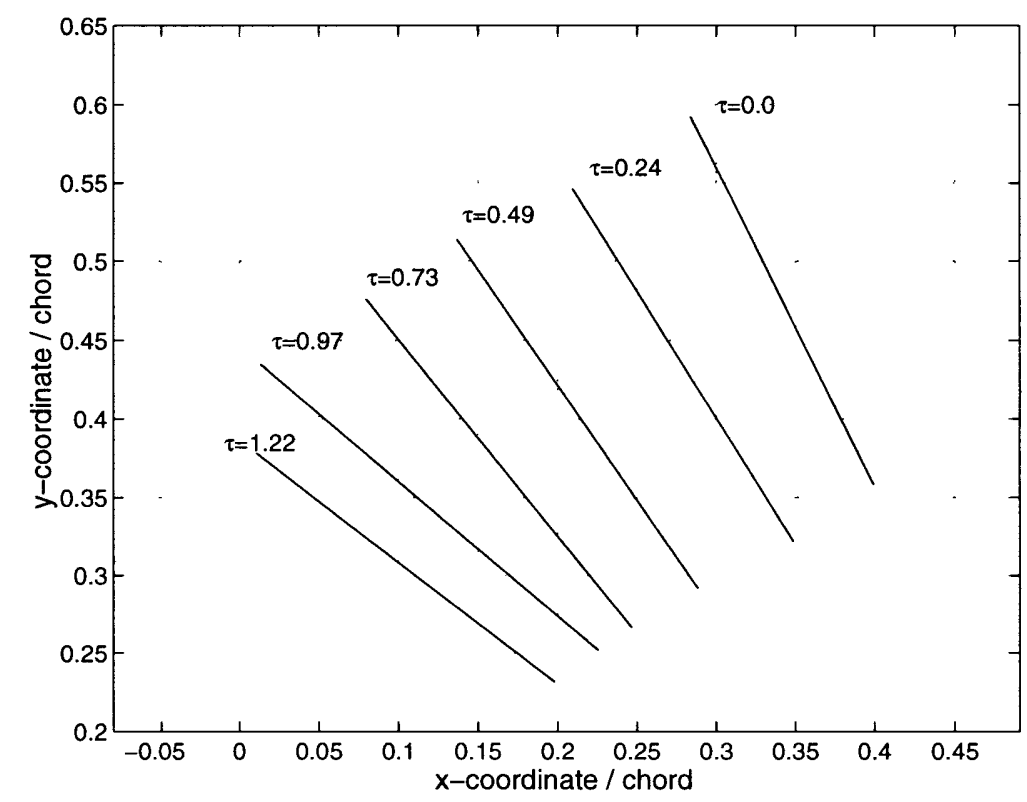

Figure 3-16: Location of upstream traveling pressure waves at specific time instances. $\tau=0.0$ is an arbitrary time instance. $M_{\infty}=0.87$.

\begin{tabular}{|c|c|c|c|}
\hline \hline Property & Lower & Mid & Upper \\
\hline Coordinates $(\mathrm{x} / \mathrm{c}, \mathrm{y} / \mathrm{c})$ & $(0.31,0.35)$ & $(0.26,0.45)$ & $(0.25,0.56)$ \\
$M_{1}$ & 0.85 & 0.77 & 0.72 \\
$\left|U / U_{\infty}\right|$ & 1.03 & 0.94 & 0.88 \\
Local sound speed $(a)$ & 1.21 & 1.22 & 1.22 \\
$\left|(U-a) / U_{\infty}\right|$ & 0.18 & 0.28 & 0.34 \\
\hline \hline
\end{tabular}

Table 3.4: Flow properties at 3 spanwise locations upstream of a pressure wave in Run 4. $M_{\infty}=$ 0.87 . 

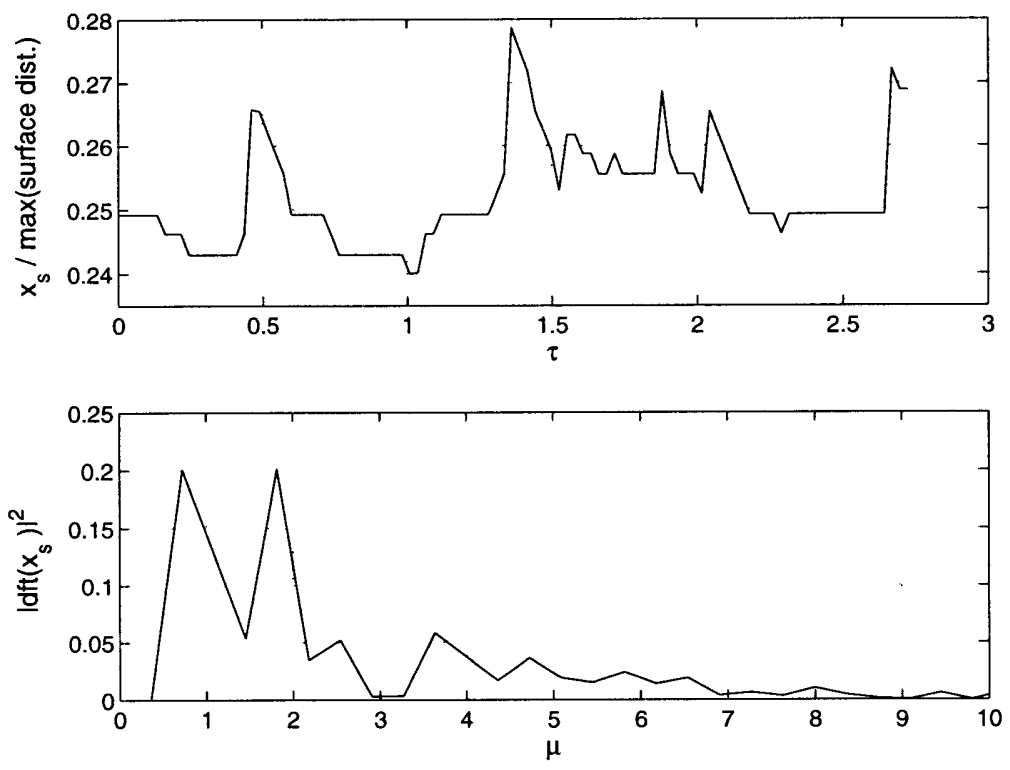

Figure 3-17: Fluctuation in the shock wave position $x_{s}$ and the corresponding frequency spectrum. Mean shock location $=0.25 \times \max$ (surf.distance). $d f t(X)$ is the discrete fourier transform of time signal $X$.

front upstream. This causes additional static pressure rise $\Delta C p_{s}$ across the shock wave. This is illustrated in Figure 3-17 and Figure 3-18 which shows (1) the fluctuation in the shock wave position on the blade surface and (2) the fluctuation in the static pressure rise $\Delta C p_{s}$ across the shock wave. $\mathrm{A} \pm 2 \%$ fluctuation in the shock wave position is seen to generate a static pressure fluctuation $\Delta C p_{s}$ of \pm 0.1 . The dark lines parallel to the shock wave in Figure 3-14 also suggests additional pressure waves generated by the fluctuation in the shock wave position.

The broader frequency band in the force and moment fluctuations alluded to earlier can be traced in part to the shock wave fluctuations. In particular the shock wave fluctuation frequency at $\mu=0.75$ can be seen in the frequency response for the Run 4 force and moments fluctuations in Figure 3-13. Additional force and moment fluctuation frequencies occur at $\mu=1.00$ and $\mu=1.50$ however. Further work is required to determine the precise origin of these additional frequencies ${ }^{3}$.

\footnotetext{
${ }^{3}$ These may be due to additional reflections of the pressure waves at the blade surfaces.
} 

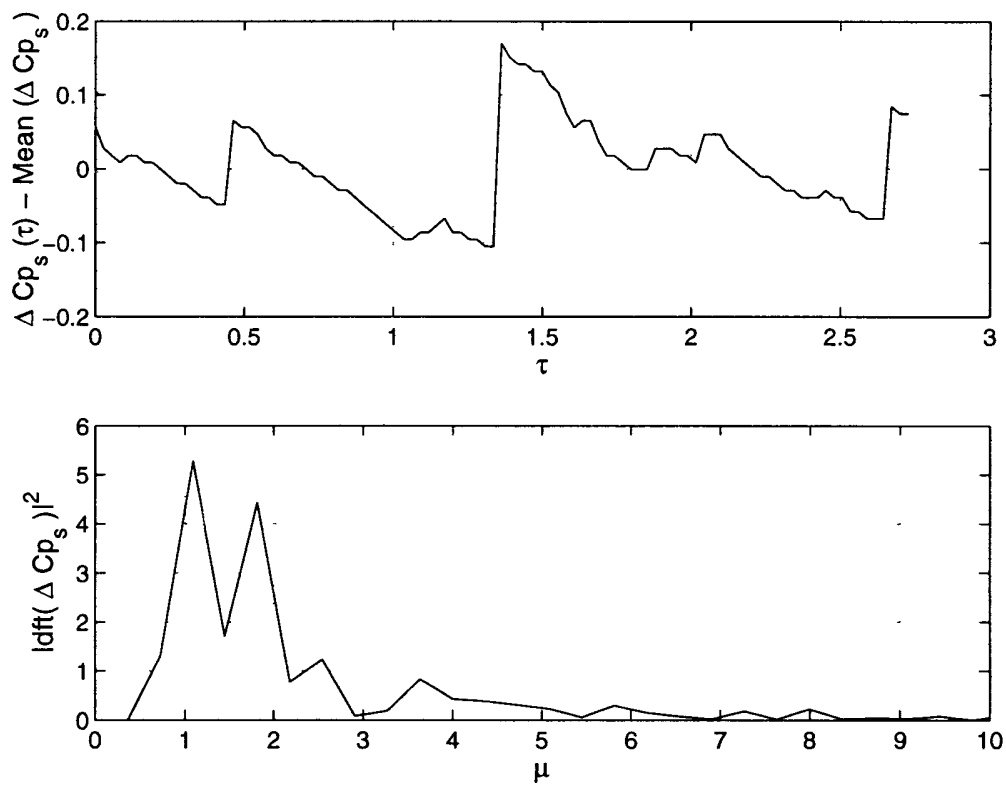

Figure 3-18: Fluctuation in the static pressure rise across the shock wave $\Delta C p_{s}$ caused by the upstream traveling pressure waves. Mean $\Delta C p_{s}=0.54 . \operatorname{dft}(X)$ is the discrete fourier transform of the time signal $X$.

\subsection{Vortex Shedding Frequency and Strouhal Number}

The vortex shedding frequencies can be expressed in terms of a non-dimensionalized Strouhal number (St) defined as,

$$
S t=\frac{n \times \text { length scale }}{\text { velocity scale }}
$$

where $n$ is the vortex shedding frequency. All the vortex shedding frequencies determined above for Runs 1-4 are in terms of Strouhal number based on the blade chord and inlet total velocity (essentially the reduced frequency). Strouhal numbers for vortex shedding have been calculated by many researchers for a number of different flow geometries and flow conditions using various length and velocity scales. For flows of aerodynamic interest "popular" Strouhal numbers have been based on the blade boundary layer thickness at the trailing edge $\delta_{b l} / c$, momentum thickness $\theta / c$, displacement thickness $\delta^{*} / c$ and blade trailing edge thickness $\vartheta$ (as length scales) and the boundary layer edge velocity (as a velocity scale). It is useful to present the vortex shedding frequencies obtained in the current research in 


\begin{tabular}{|c|c|c|c|c|}
\hline \hline Strouhal number & Run 1 & Run 2 & Run 3 & Run 4 \\
\hline$M_{\infty}$ & 0.15 & 0.53 & 0.63 & 0.87 \\
$\operatorname{St}\left(c, U_{\infty}\right)$ & 3.5 & 3.0 & 2.9 & 1.9 \\
$\operatorname{St}\left(\delta_{b l}, U_{\text {edge }}\right)$ & 0.55 & 0.44 & 0.43 & 0.36 \\
$\operatorname{St}\left(\theta, U_{\text {edge }}\right)$ & 0.03 & 0.02 & 0.02 & 0.01 \\
$\operatorname{St}\left(\delta^{*}, U_{\text {edge }}\right)$ & 0.11 & 0.12 & 0.13 & 0.29 \\
$\operatorname{St}\left(\vartheta, U_{\text {edge }}\right)$ & 0.08 & 0.07 & 0.06 & 0.04 \\
$\operatorname{St}\left(\vartheta+\delta^{*}, U_{\text {edge }}\right)$ & 0.19 & 0.18 & 0.19 & 0.34 \\
\hline \hline
\end{tabular}

Table 3.5: Vortex shedding frequencies expressed in terms of Strouhal number for each Mach number flow.

terms of these other definitions to allow convenient comparisons to be made.

The vortex shedding frequencies expressed in terms of these different Strouhal number definitions are listed in Table 3.5 for each Mach number flow. The values for boundary layer displacement thickness and momentum thickness required to calculate these Strouhal numbers are obtained from the time averaged boundary layer properties plotted in Figure A-9 through Figure A-12 in Appendix A.

There is a large difference in the numerical values for Strouhal number at each flow Mach number depending on the particular definition used. Based on results from several researchers Gostelow [6] notes that Strouhal number (based on the blade trailing edge thickness) for vortex streets behind cascades of aerofoils should lie in the range $0.1-0.4$. Table 3.5 however indicates the Strouhal number based on the blade trailing edge thickness to be an order of magnitude smaller than this range. This discrepancy is due to the blade boundary layer which increases the effective trailing edge thickness by an amount $\delta^{*}$. The Strouhal numbers based on the sum of the trailing edge thickness $\vartheta$ and displacement thickness $\delta^{*}$ (of the blade suction surface) which now takes into account the effective trailing edge thickness varies between $0.18-0.34$ and is more in line with the values quoted by Gostelow.

Note the pressure surface displacement thickness (at the blade trailing edge) is an order of magnitude smaller than the corresponding suction surface displacement thickness (see Figure A-9 through Figure A-12) and has therefore not been included in the evaluation for 
Strouhal number $\operatorname{St}\left(\vartheta+\delta^{*}, U_{\text {edge }}\right)$.

The Strouhal number based on trailing edge thickness and displacement thickness show small variations $(5 \%)$ with Mach number for the $M_{\infty}=0.15,0.53$ and 0.63 flows. For flow at $M_{\infty}=0.87$ however the Strouhal number increases by over $100 \%$ from the Strouhal numbers at the lower Mach numbers. A similar trend is seen in the experimental results of Heinemann and Butefisch [8] for the flow through turbine cascades. This large increase in Strouhal number (due to the large increase in displacement thickness) is most likely a compressibility effect [4].

\subsection{Summary}

The force and moment fluctuations induced by vortex shedding have been quantified for baseline flows ranging from $M_{\infty}=0.15$ to $M_{\infty}=0.87$. A summary of the key results are listed below.

- The amplitude of the vortex shedding induced blade force and moment fluctuations increase with free stream Mach number. The fluctuation magnitudes about the time averaged mean values are as follows:

1. $C y: \pm 1 \%$ at $M_{\infty}=0.15$ to $\pm 5 \%$ at $M_{\infty}=0.87$.

2. $C x: \pm 1 \%$ at $M_{\infty}=0.15$ to $\pm 3 \%$ at $M_{\infty}=0.87$.

3. $C m: \pm 2 \%$ at $M_{\infty}=0.15$ to $\pm 13 \%$ at $M_{\infty}=0.87$.

- Vortex shedding for the $M_{\infty}=0.87$ flow generates significant pressure waves at the trailing edge. These pressure waves travel upstream and cause $\pm 2 \%$ amplitude fluctuations in the shock wave position and a corresponding static pressure fluctuation across the shock wave $\Delta C p_{s}$ of \pm 0.1 .

- The frequency of the baseline force and moment compare closely with the vortex shedding frequencies. 
- The Strouhal number for vortex shedding based on the blade trailing edge thickness and boundary layer displacement thickness lie in the range $0.18-0.34$.

The fluctuation magnitudes obtained in this Chapter indicate that vortex shedding is an additional possible source for high cycle fatigue failure. 


\section{Chapter 4}

\section{Viscous Results: Response to DENSITY WAKES}

The aim of this Chapter is to characterize the force and moment fluctuations induced by convecting density wakes in viscous compressible flows.

The density wake induced force and moment response profiles in viscous compressible flows are found to consist of 3 distinct regions each characterized by separate flow features. The flow features in each region are first identified with the aid of density and Mach number contour plots. The trends in the magnitude and frequency of the fluctuations are then determined for a range of density wake properties and flow Mach numbers. Table 4.1 summarizes the variables used to characterize these trends. In each case the density wakes are convected past the LSRC cascade blade row described in Section 2.3.

\begin{tabular}{|c|c|c|c|c|}
\hline \hline Parameter & Run 1 & Run 2 & Run 3 & Run 4 \\
\hline$M_{\infty}$ & 0.15 & 0.53 & 0.63 & 0.87 \\
\hline$w / c$ & $0.1,0.2,0.4,1.0$ & \multicolumn{3}{|c|}{$0.1,0.2,0.4$} \\
\hline$\rho_{2} / \rho_{1}$ & \multicolumn{3}{|c|}{$0.25,0.50,0.75,2.00$} \\
\hline \hline
\end{tabular}

Table 4.1: Parametric test variables. $w / c=$ non-dimensional wake width, $\rho_{2} / \rho_{1}=$ maximum density inside wake / free stream density. 


\subsection{Force and Moment Profiles: Initial characterization}

The density wake induced force and moment response profiles in viscous compressible flows consist of 3 distinct regions. These regions are illustrated in Figure 4-1 during the passage of a density wake of width $0.2 c$ and density ratio $\rho_{2} / \rho_{1}=0.5$ at free stream Mach number 0.15. The position of the density wake at specific time instances are listed in Table 4.2. The characteristic flow features in each response region are summarized below.

1. Baseline response region. This response corresponds to all times when the density wake is upstream of the compressor blade leading edge. The force and moment fluctuations observed here are characterized by vortex shedding at the blade trailing edge. Note vortex shedding is a consequence of the viscous flow environment and is not related to the passage of density wakes.

2. Primary response region. This response corresponds to when the density wake is within the cascade blade passage. The characteristic flow features here are the fluid flux directed to the blade surfaces and the associated counterrotating vortices ${ }^{1}$. The shock wave fluctuation is also an additional feature for higher Mach number flows $\left(M_{\infty}=0.87\right)$.

3. Secondary response region. This response corresponds to all times after the density wake leaves the compressor blade trailing edge. This response region is characterized by a separation bubble on the blade suction surface formed by the density wake boundary layer interaction.

The primary and secondary response will be discussed with reference to the characteristic flow features listed above in the subsequent sections. The parametric variables governing the force and moment fluctuations and the trends in the maximum fluctuations with increasing flow Mach number will also be described. The baseline response fluctuations have already been discussed in Chapter 3 and will not be repeated here.

\footnotetext{
${ }^{1}$ Note these are the same features as for inviscid incompressible flows.
} 

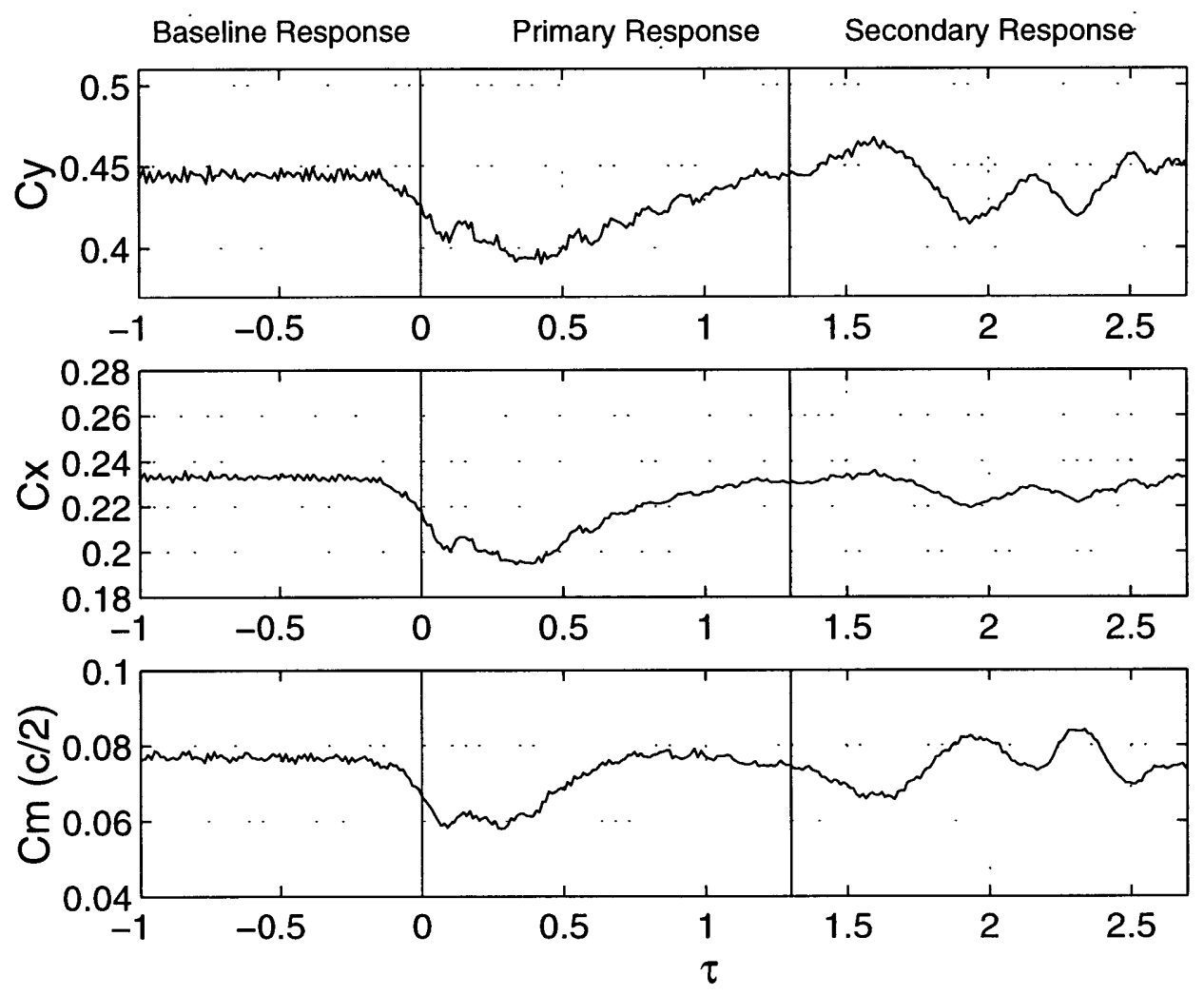

Figure 4-1: Fluctuations in (1) azimuthal force coefficient, (2) axial force coefficient and (3) moment coefficient (positive clockwise about the mid chord) during passage of a density wake of width $0.2 c$ and density ratio $\rho_{2} / \rho_{1}=0.5$. 3 distinct regions can be identified in the response. $M_{\infty}=0.15$.

\begin{tabular}{|c|l|}
\hline \hline Convective Time Scale $(\tau)$ & Location of density wake \\
\hline 0.0 & wake l.e. intercepts blade l.e. \\
0.1 & wake t.e. intercepts blade l.e. \\
1.3 & wake t.e. passes blade t.e. \\
\hline \hline
\end{tabular}

Table 4.2: The location of a density wake at different times during passage through the LSRC cascade blade row. $w / c=0.2, \rho_{2} / \rho_{1}=0.50, M_{\infty}=0.15$. 


\subsection{Primary Response}

\subsubsection{General Flow Features}

The density contour images in Figure 4-2 through Figure 4-5 indicates the passage of a density wake of width $0.2 c$ and density ratio 0.5 at free stream Mach number 0.15 through the LSRC cascade blade row. The density wake is seen to deform from an initial planar profile to an approximately triangular shape profile during passage through the blade row. This deformation is a result of the convection of low density, wake fluid to the blade suction surface by the action of centrifugal forces (Section 1.2). A pair of counterrotating vortices are also formed in the blade passage identical to that observed in the inviscid flow simulations (Figure 1-4). Gradual counterclockwise skewing of the wake can also be observed. This is a result of the higher flow velocity near the blade suction surface than at the blade pressure surface.

The transport of the low density wake fluid to the blade suction surface constitutes a fluid flux which can be expressed mathematically as,

$$
\text { fluid flux }=\int_{0}^{w / c} \rho v_{f} d x
$$

where $\rho$ is the wake density, $v_{f}$ is the velocity of the wake fluid and $d x$ is a increment in length parallel to the axial flow direction. $\rho$ and $v_{f}$ are functions of $x . v_{f}$ and wake width $w$ are also functions of time $\tau$. The influence of this fluid flux on the blade force and moment fluctuations is described next.

The impingement of the low density fluid flux on the blade suction surface results in a local increase in the blade static pressure. As the density wake convects downstream, the local increase in static pressure moves further aft along the blade suction surface. This effect can be seen in the suction surface pressure distribution plotted in Figure 4-6. A 


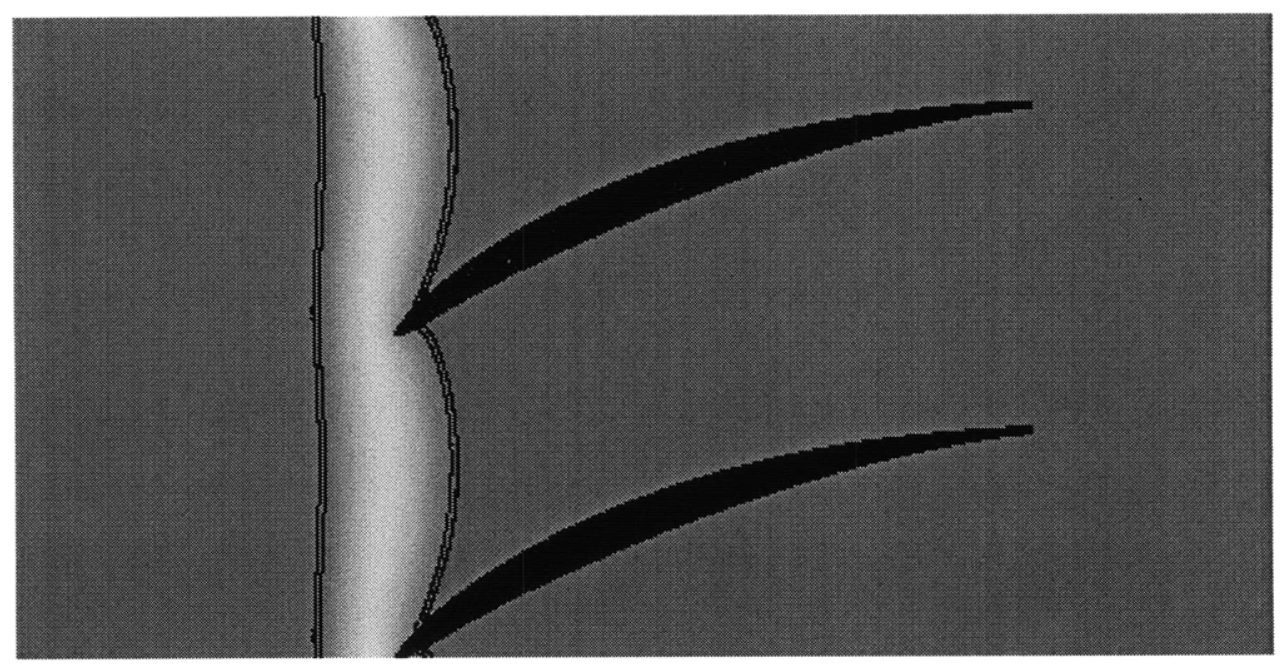

Figure 4-2: Density contour image showing passage of density wake of width $0.2 c$ and density ratio $0.5 . M_{\infty}=0.15 . \tau=0.04$.

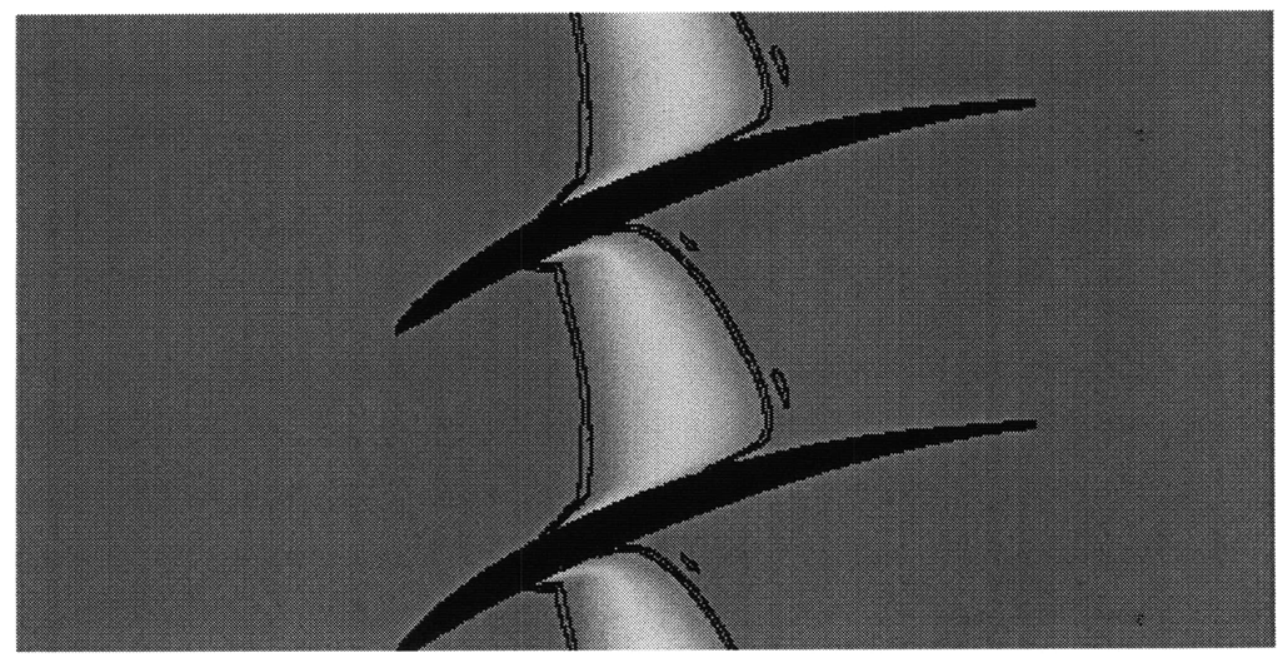

Figure 4-3: Density contour image showing passage of density wake of width $0.2 c$ and density ratio $0.5 . M_{\infty}=0.15 . \tau=0.53$. 


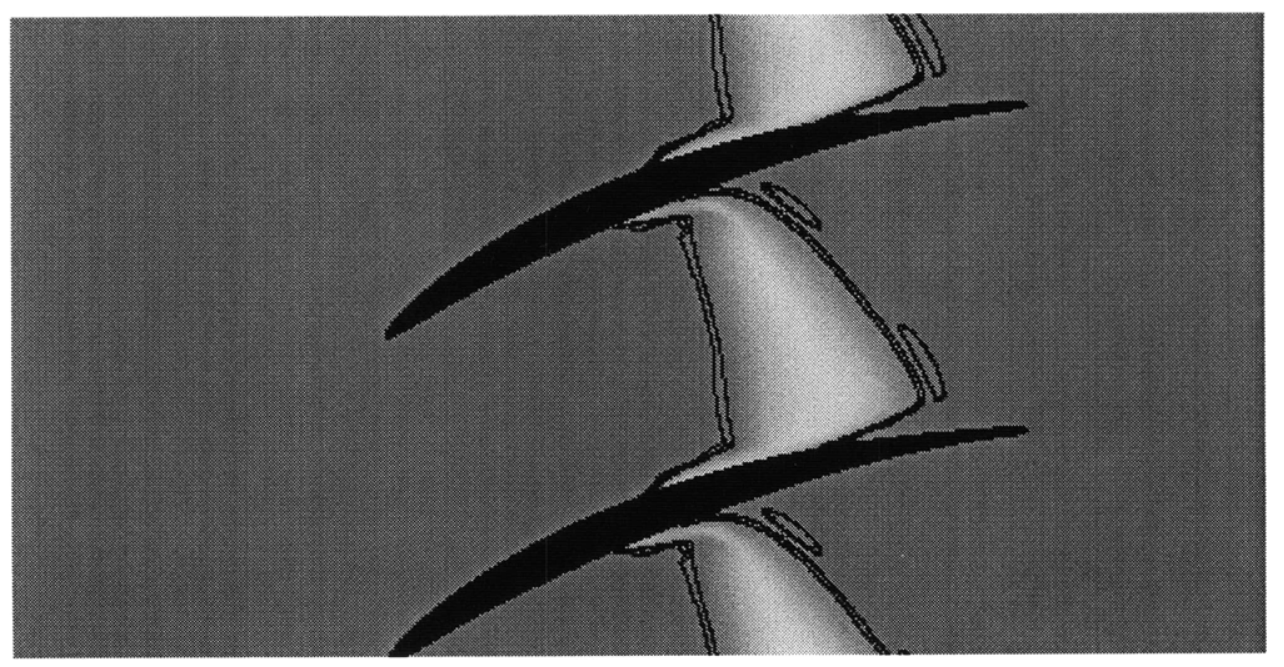

Figure 4-4: Density contour image showing passage of density wake of width $0.2 c$ and density ratio $0.5 . M_{\infty}=0.15 . \tau=0.78$.

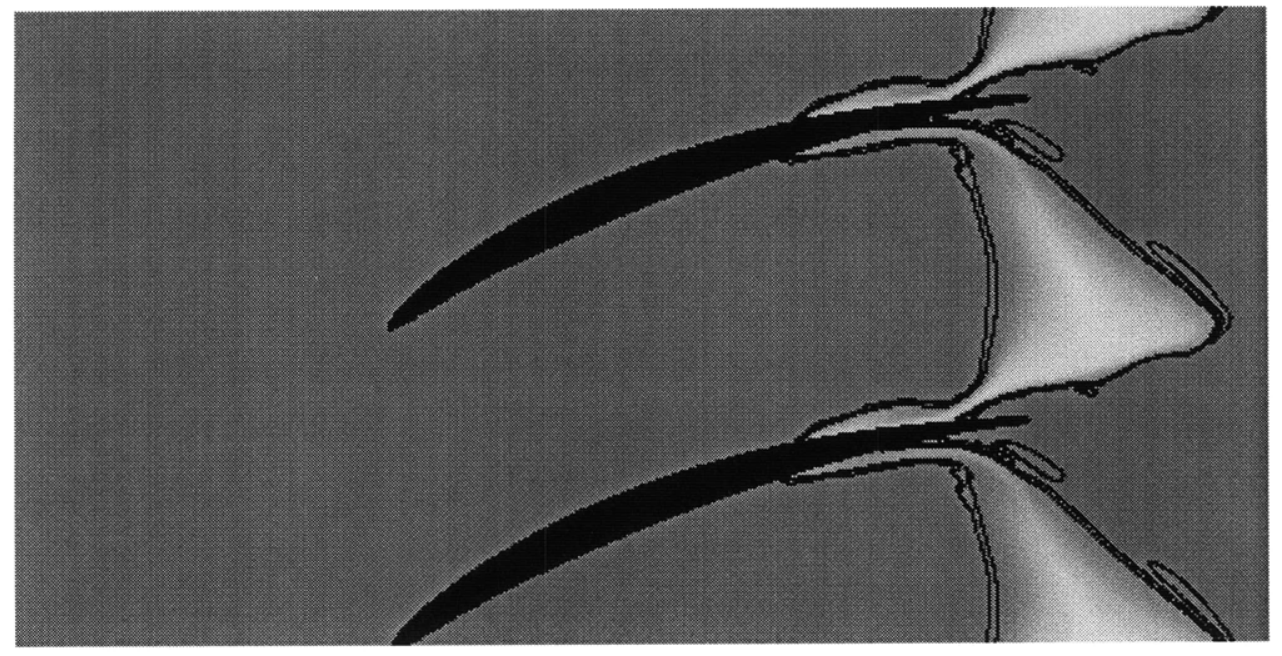

Figure 4-5: Density contour image showing passage of density wake of width $0.2 c$ and density ratio $0.5 . M_{\infty}=0.15 . \tau=1.28$. 
corresponding decrease in static pressure occurs at the blade pressure surface due to the "suction" effect of the counterrotating vortices. The net result is a local reduction in the static pressure difference across the blade as shown in Figure 4-7.

The magnitude of the fluid flux and the corresponding local reduction in blade static pressure difference therefore governs the magnitude of the force and moment fluctuations in the primary response. In general the magnitude of the fluid flux increases as the density wake width increases and the velocity $v_{f}$ of the fluid increases. The latter is determined by the strength of the vorticity produced in the blade row as described by Equation 1.3. Larger pressure gradients (across the blade row) and larger density gradients both increase the blade row vorticity and hence also the fluid velocity $v_{f}$. The effect of wake width and density ratio on the magnitude of the force and moment fluctuations are discussed separately in Section 4.2.3.

The blade pressure distribution in the primary response for Run 4 is also influenced by fluctuations in the shock wave location on the blade surface. This is examined in the next section.

\subsubsection{Run 4: $\mathrm{M}_{\infty}=0.87$}

Run 4 is characterized by a shock wave located $0.25 \mathrm{c}$ downstream of the blade leading edge as shown in Figure 3-10. During the passage of a low density wake the shock wave is found to move upstream towards the blade leading edge (for a high density wake the shock wave is found to move downstream). During this motion the static pressure rise $\Delta C p_{s}$ across the shock wave is found to increase. This is illustrated in Figure 4-8 during the passage of a density wake of width $0.1 c$ and density ratio 0.25 . The change in the blade azimuthal force coefficient is also shown here. Notice the maximum reduction in azimuthal force coefficient corresponds to the point of maximum static pressure rise across the shock wave. The same is true for the axial force coefficient and the moment coefficient. 


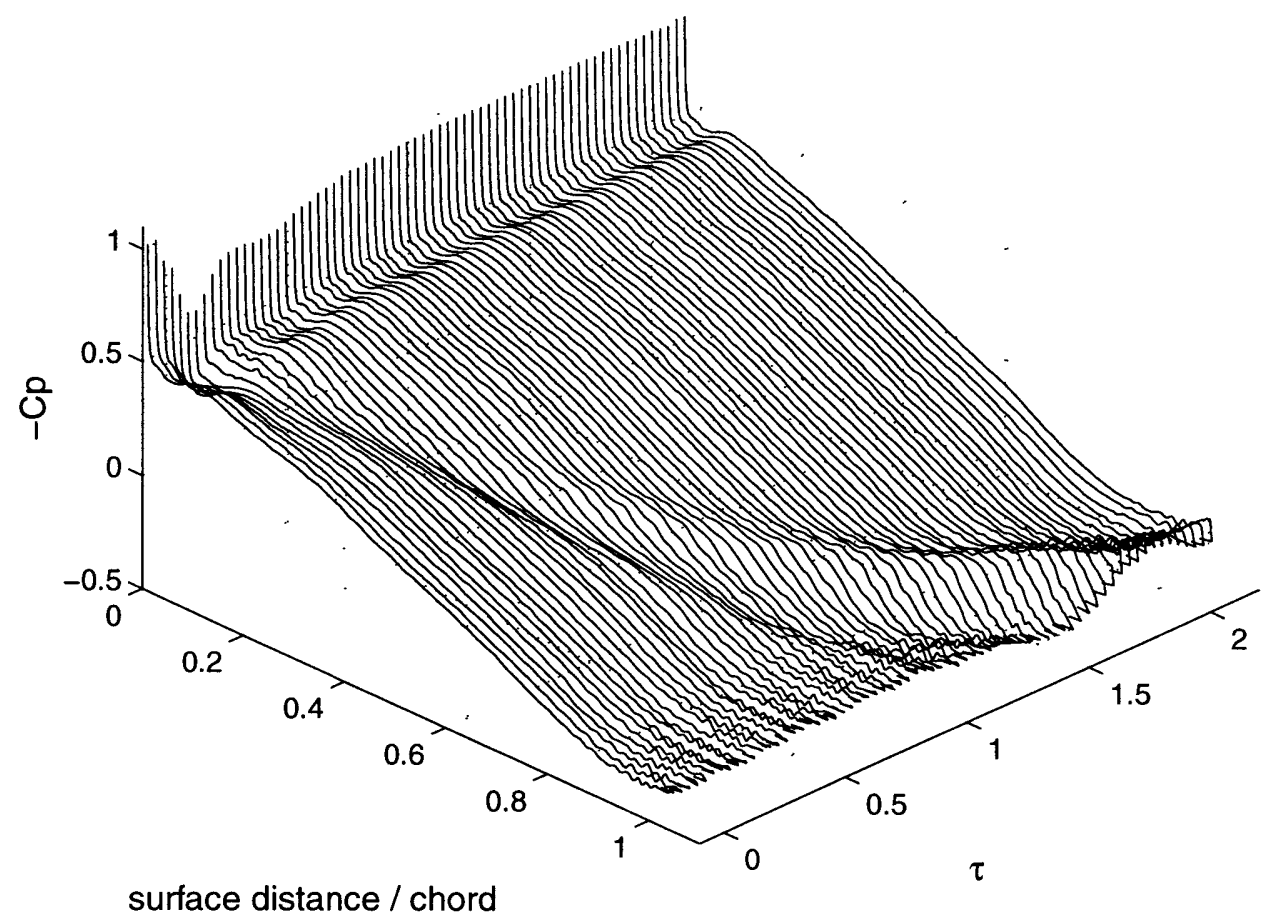

Figure 4-6: Suction surface pressure distribution during passage of a density wake width $0.2 c$ and density ratio $0.5 . M_{\infty}=0.15$.

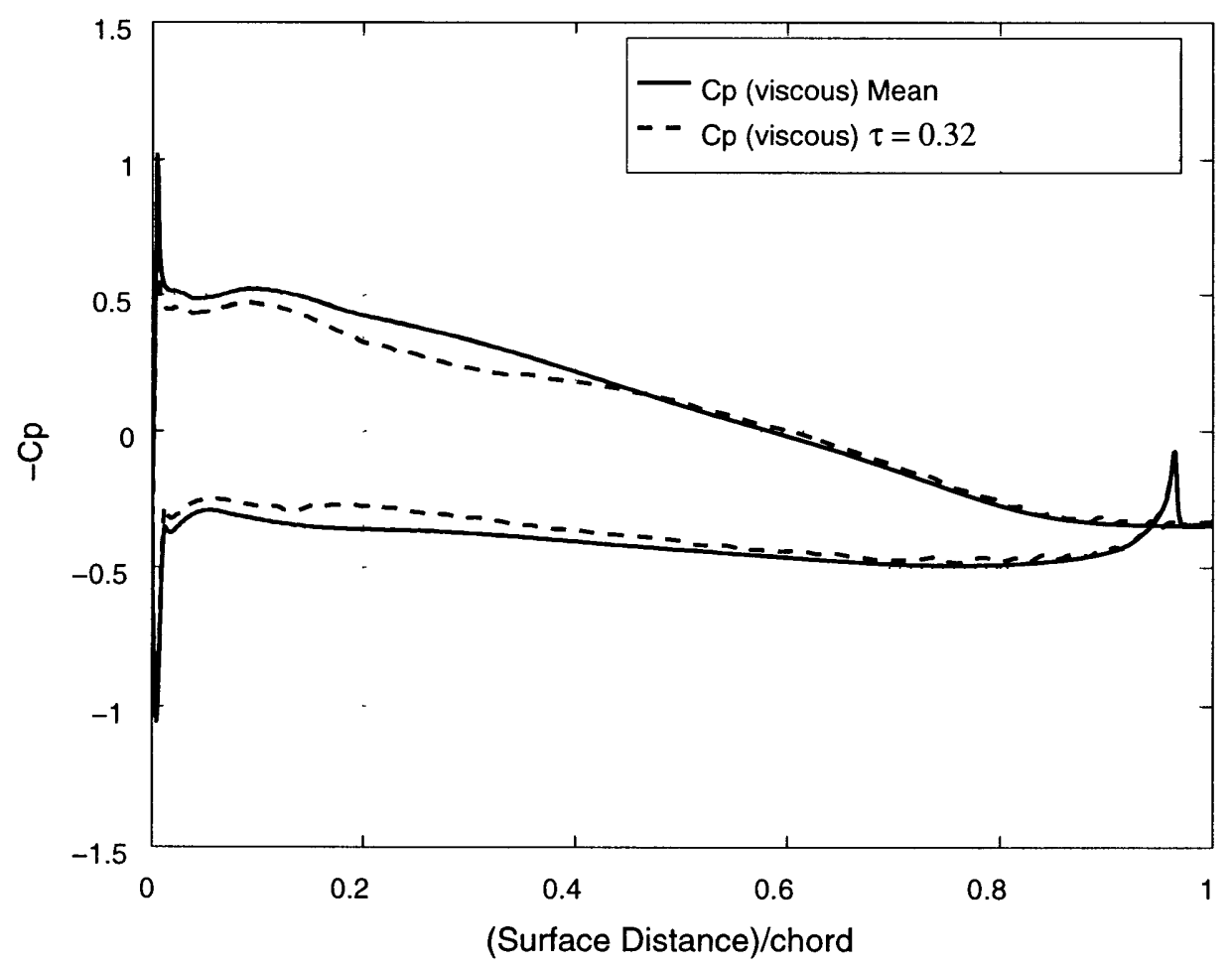

Figure 4-7: Change in blade pressure coefficient during passage of a density wake width $0.2 c$ and density ratio $0.5 . \tau=0.32, M_{\infty}=0.15$. 
The change in static pressure difference across the shock wave is affected by several sources. These source are listed below and quantified in Table 4.3.

1. The fluid flux towards the suction surface. The effect of the fluid flux is to increase the blade suction surface static pressure (Section 4.2.1). When the fluid flux is directed ahead of the shock wave $\Delta C p_{s}$ is reduced (since the static pressure upstream of the shock wave increases). When the fluid flux is directed behind the shock wave $\Delta C p_{s}$ is increased (since the static pressure downstream of the shock wave increases).

2. Upstream traveling pressure waves. These pressure waves increase $\Delta C p_{s}$ as they intercept the shock wave (see Figure 3-18 and the discussion in Section 3.1.4).

3. Lower local Mach number within the density wake. For low density wakes, the Mach number within the density wake is lower on account of the higher local temperature (and therefore higher local speed of sound). In general as the upstream Mach number decreases the static pressure ratio across the shock wave also decreases (Rankine Hugoniot relations).

4. The upstream motion of the shock wave. The shock wave moves upstream by $0.07 c$ during interaction with a low density wake as shown in Figure 4-8. During this upstream motion the shock wave relative upstream Mach number increases. This results in an increase in the static pressure rise across the shock wave (Rankine Hugoniot relations). The opposite is true when the shock wave moves downstream.

The estimates listed in Table 4.3 are specific to the passage of a density wake of width $0.1 c$ and density ratio 0.25 . These estimates should be considered at best orderof-magnitude accurate. The estimates are constrained to sum to the maximum value of the static pressure difference ( 0.8 for this case).

While the fluid flux of the counterrotating vortices contribute to $63 \%$ of the overall increase in static pressure rise across the shock wave, the contribution by the shock motion is almost a factor of 5 greater. The shock wave motion is therefore the more dominant mechanism which governs the shock wave static pressure rise and hence the fluc- 


\begin{tabular}{|c|c|c|}
\hline \hline Source & Estimated $\Delta C p_{s}$ & $\%$ of $\max . \Delta C p_{s}$ \\
\hline Fluid flux & +0.50 & +63 \\
Pressure waves & +0.20 & +25 \\
Lower Mach no. & -2.40 & -300 \\
Shock motion & +2.50 & +312 \\
\hline SUM & +0.80 & +100 \\
\hline \hline
\end{tabular}

Table 4.3: Estimated contribution of several sources to the maximum pressure difference across the shock wave. Estimated values are specific to the passage of a density wake of width $0.1 c$ and density ratio $0.5 . M_{\infty}=0.87$.

tuations in the force and moment coefficients for the $M_{\infty}=0.87$ flow.

An interesting situation arises during passage of a density wake of width $0.4 c$ and density ratio 0.25 . As the density wake convects over the leading edge of the blade the local supersonic flow "switches" to a subsonic flow on account of the lower local Mach number (due to the higher speed of sound) within the density wake. The blade shock wave is then temporarily suppressed from the flow. This feature can be seen in the pressure contour plot in Figure 4-9. The dark band at $x / c=0.25$ represents the shock wave front. This front disappears at $\tau \approx 0.4$ as the density wake convects over the leading edge of the blade. The shock wave reappears at $\tau \approx 1.2$ as the density wake convects further downstream. A similar result is observed during the passage of a density wake of width $0.2 c$ and density ratio 0.25 . These values for density ratio and density wake width provide an upper bound to the maximum upstream motion of the shock wave. This is discussed below.

The maximum deflection of the shock wave for a range of density wake properties is plotted in Figure 4-10. Note for a high density wake (wake density higher than free stream density) the shock wave moves downstream. In general the shock wave displacement increases with density wake width and density parameter $\left|\rho^{*}\right|$. The data points for density parameter $\rho^{*}=-0.60$ and wake widths $0.2 c$ and $0.4 c$ indicate the last recorded position of the shock wave before it is suppressed by the lower Mach number within the density wake. The maximum deflection of the shock wave is found to vary almost linearly for values of density parameter $\rho^{*} \leq-0.15$. The trends seen here for the shock wave deflection and the associated variations in the blade force and moment coefficients will be discussed in the 

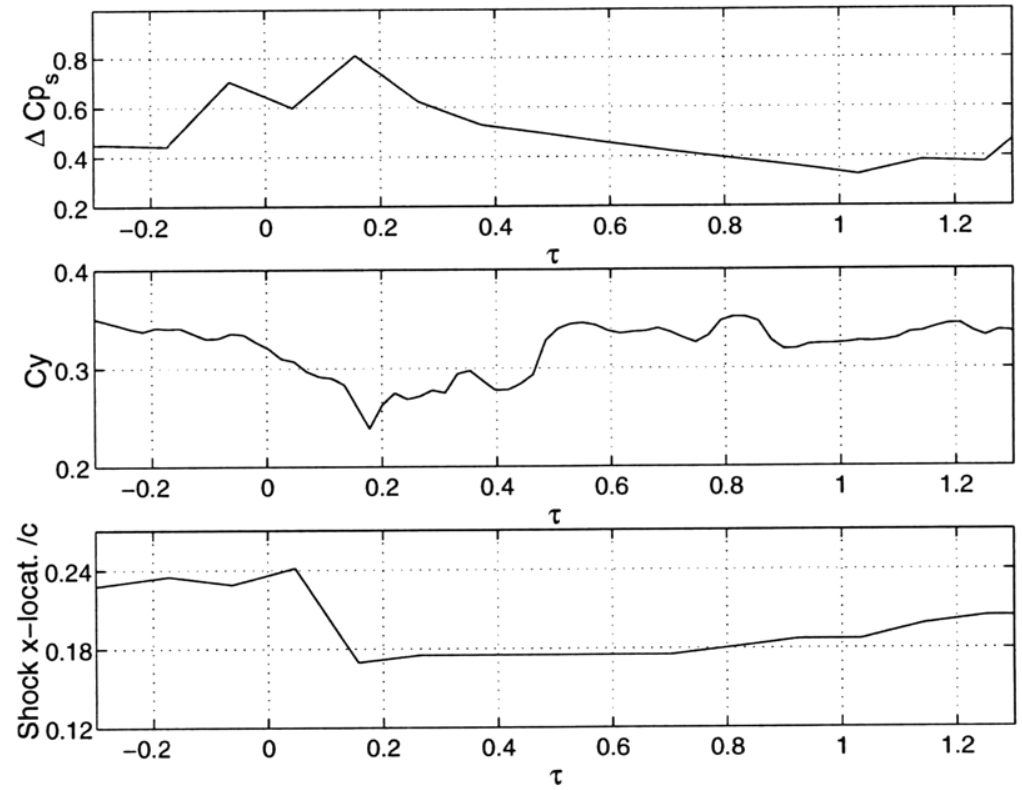

Figure 4-8: The change in (1) static pressure difference across the shock wave, (2) azimuthal force coefficient and (3) blade shock location during passage of a density wake width $0.1 c$ and density ratio $0.25 . M_{\infty}=0.87$.

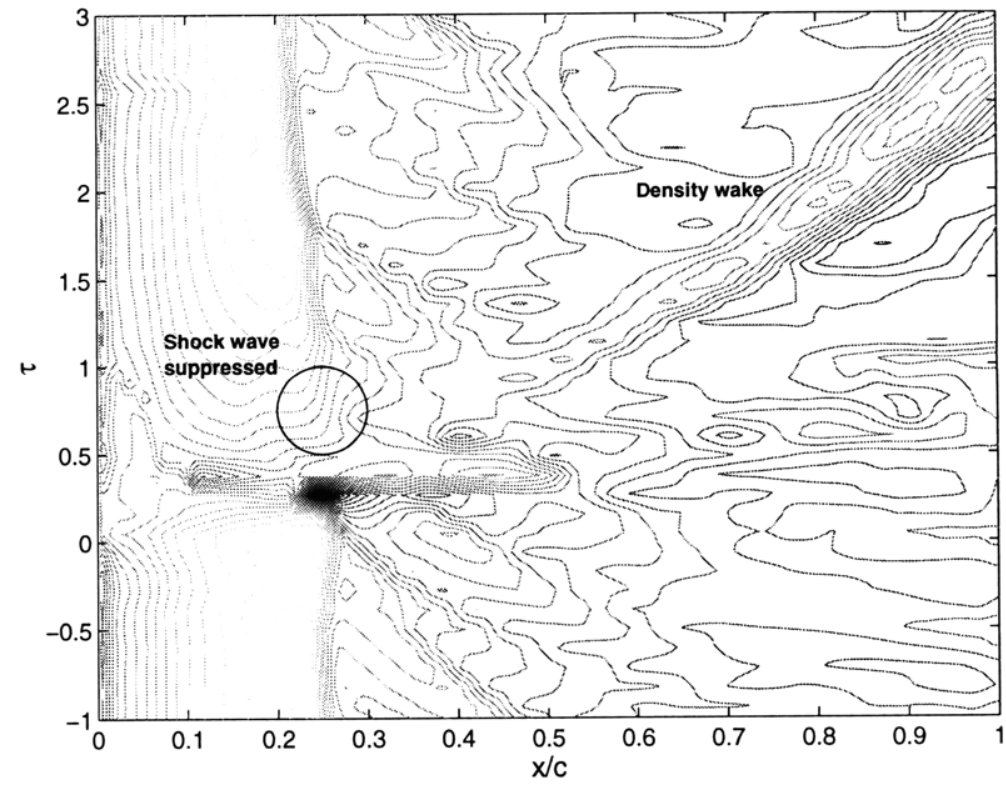

Figure 4-9: Blade suction surface pressure contours showing the temporary suppression of the blade passage shock wave during passage of a density wake of width $0.4 c$ and density ratio 0.25 . The dark band at $x / c=0.25$ is the shock front. $M_{\infty}=0.87$. 


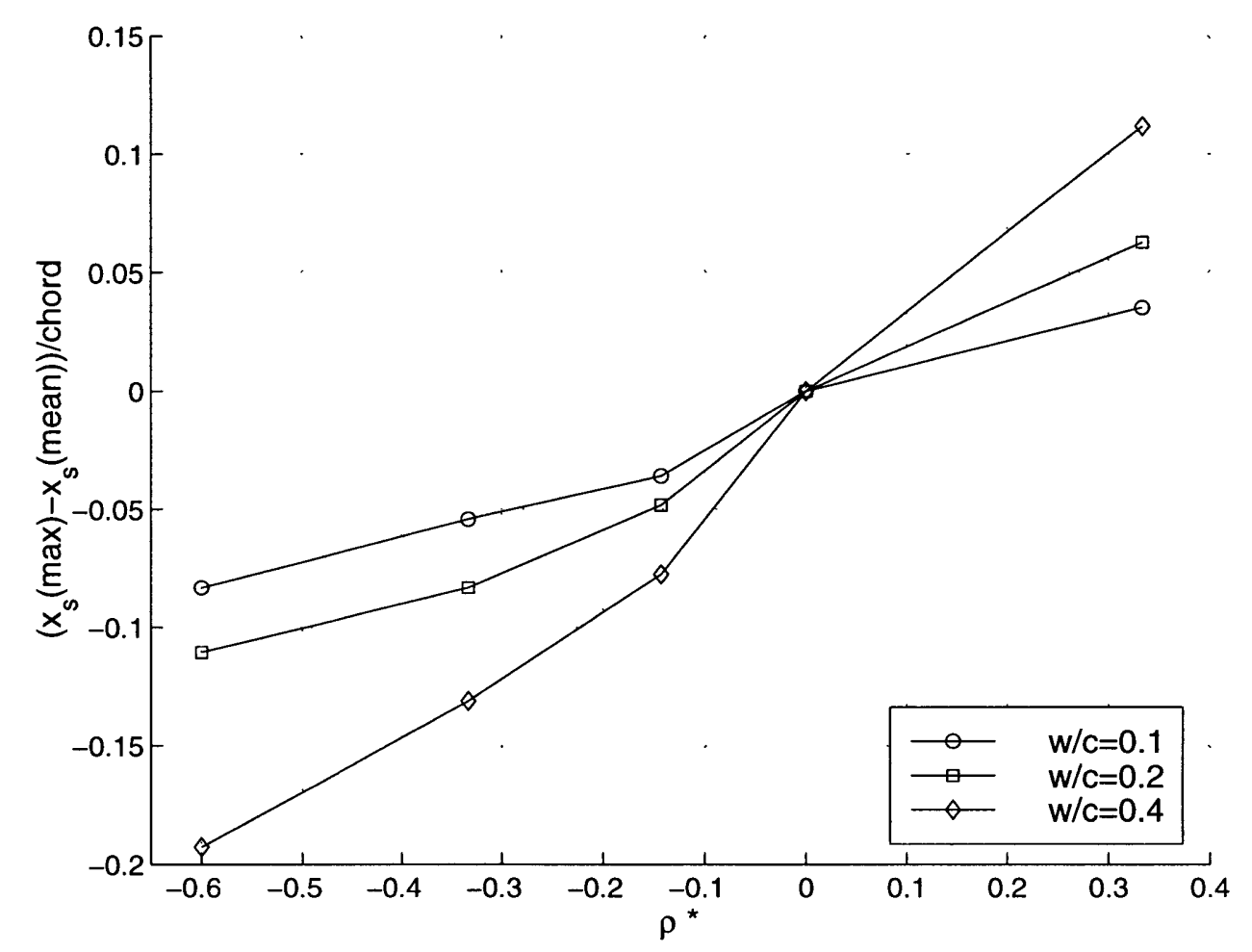

Figure 4-10: Changes in the maximum deflection of the shock wave as a function of the density wake width $w / c$ and density parameter $\rho^{*}$.

next section.

\subsubsection{Parametric Study}

A parametric study was conducted to establish the trends and quantify the effect of free stream Mach number, density wake width and density ratio on the maximum amplitude of the blade force and moment fluctuations. The complete parametric calculation matrix is tabulated in Table 4.1. The time varying blade force and moment fluctuations are plotted in Figure B-1 through Figure B-52 in Appendix B for each simulation.

The fluctuation in the force and moment coefficients caused by vortex shedding is superimposed on the density wake induced force and moment fluctuations throughout the primary response region. In most cases the maximum fluctuation in the primary response region can be easily distinguished from the baseline response. However as the free stream 
Mach number increases the baseline fluctuations increase in amplitude and can sometimes exceed the primary response fluctuations. This is particularly true during the passage of density wakes with small density ratios $\left(\rho_{2} / \rho_{1}=0.75\right)$ at high free stream Mach numbers. A clear distinction between the baseline response and the primary response cannot be seen for these cases. The maximum fluctuation in the primary response is nevertheless obtained by identifying the maximum (or minimum) point on the response curves corresponding to the time when the density wake is contained within the blade passage.

The maximum fluctuation in the force and moment coefficients obtained in this manner are plotted in Figure 4-11 through Figure 4-14 as percentage changes from the time averaged baseline values. Negative values indicate a decrease from the baseline value. All results are plotted against Marble's density parameter $\rho^{*}$ and density wake width $w / c$. Note there is an uncertainty associated with the maximum fluctuations from the mean values which corresponds to the baseline fluctuation magnitudes listed in Table $3.2^{2}$. Several trends in the primary response results are noted below.

- The magnitude of the fluctuations increase with density wake width $w / c$ and the density parameter $\left|\rho^{*}\right|$.

- The change in the magnitude of the fluctuations become increasingly non-linear for large wake widths $w / c \geq 0.2$ and large density parameters $\left|\rho^{*}\right| \geq 1 / 3$.

- The magnitude of the fluctuations induced by high density wakes (wake density higher than free stream density, $\rho^{*} \geq 0$ ) are in general larger than the magnitude of the fluctuations induced by low density wakes (wake density lower than free stream density, $\left.\rho^{*} \leq 0\right)$ for a given wake width.

As noted earlier the magnitude of the fluid flux at the blade surface increases as the density wake width and density parameter increases. The increase in the maximum fluctuation in the force and moment coefficients with wake width and density parameter in Figure 4-11 through Figure 4-14 are therefore as expected. The larger fluctuations observed

\footnotetext{
${ }^{2}$ This uncertainty must be kept in mind when evaluating the value for the numerical difference between the data points.
} 
for $\rho^{*}>0$ is due to the greater momentum transfer from the high density wakes to the blade (due to the greater mass of the density wake). The physical reason for the non-linearity in the fluctuations with increasing wake width and density parameter is unclear however.

The trends for the viscous compressible simulations are similar to the trends observed for the inviscid incompressible simulations. This is not surprising for the $M_{\infty}=0.15$, $M_{\infty}=0.53$ and $M_{\infty}=0.63$ flow cases since the same basic mechanism as for the inviscid flow (which consists of the fluid flux directed toward the blade surfaces) is responsible for the changes in the blade static pressure distribution. For the $M_{\infty}=0.87$ calculation however additional changes in the blade static pressure distribution occur due to the unsteady shock wave motion (Section 4.2.2). The trends in the force and moment coefficients nevertheless remain unchanged. This can also be expected since the magnitude of the shock wave motion and hence blade static pressure distribution follow the same trends with changes in the density wake width and density parameter (see Figure 4-10). Note however the change in magnitude of the force and moment fluctuations at $M_{\infty}=0.87$ have a more linear profile than the corresponding results at the lower Mach numbers. This compares with the nearlinear variation of the magnitude of the shock wave motion with density parameter shown in Figure 4-10.

\section{Functional Relationships}

Functional relationships can be derived to help quantify the trends stated in the previous section. The change in the maximum fluctuation of the force and moment coefficients with density parameter $\rho^{*}$ can be expressed by the following algebraic relationships, 

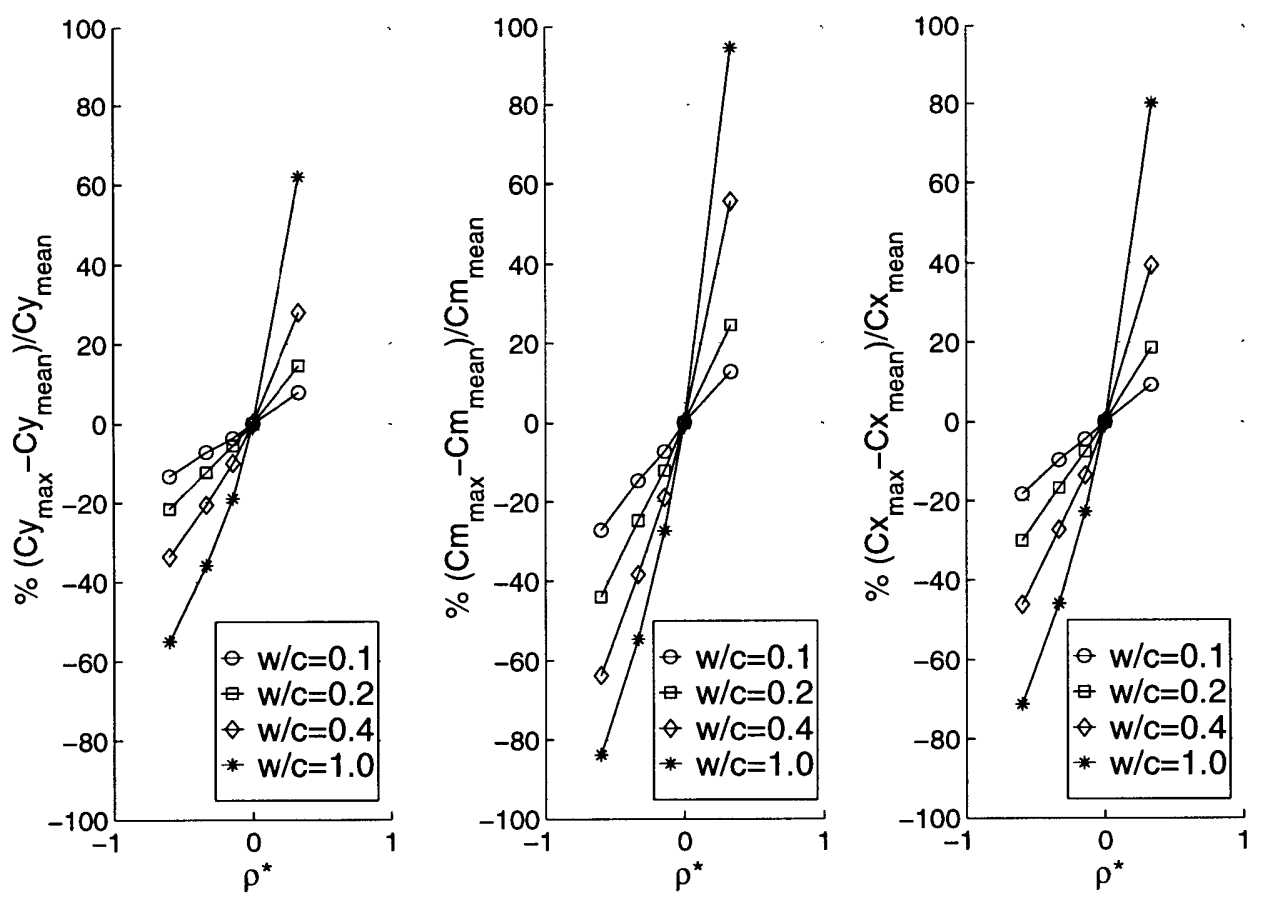

Figure 4-11: Maximum fluctuation in the force and moment coefficients as functions of density wake width and density parameter $\rho^{*}$. The straight lines joining data points are to help aid clarity. $M_{\infty}=0.15$.
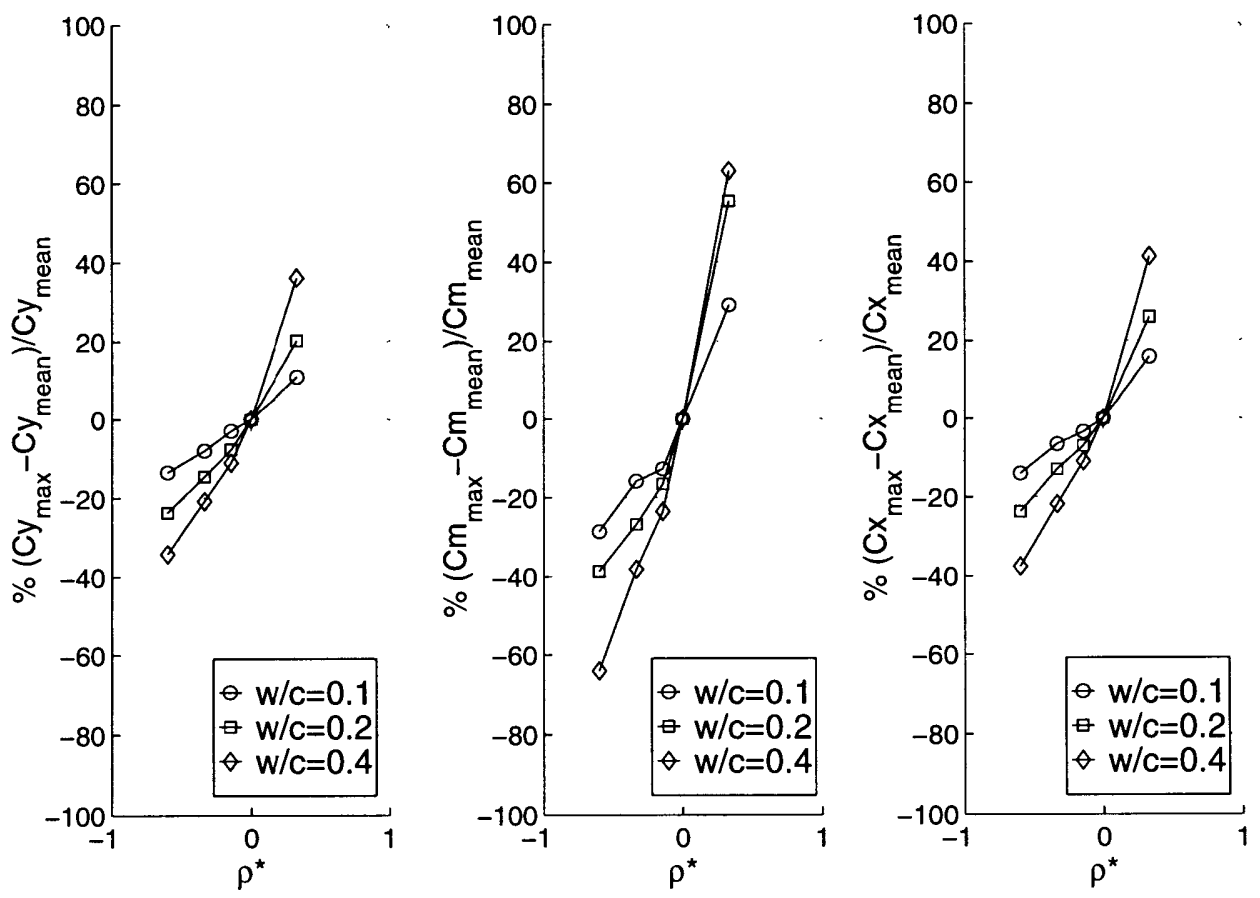

Figure 4-12: Maximum fluctuation in the force and moment coefficients as functions of density wake width and density parameter $\rho^{*}$. The straight lines joining data points are to help aid clarity. $M_{\infty}=0.53$. 

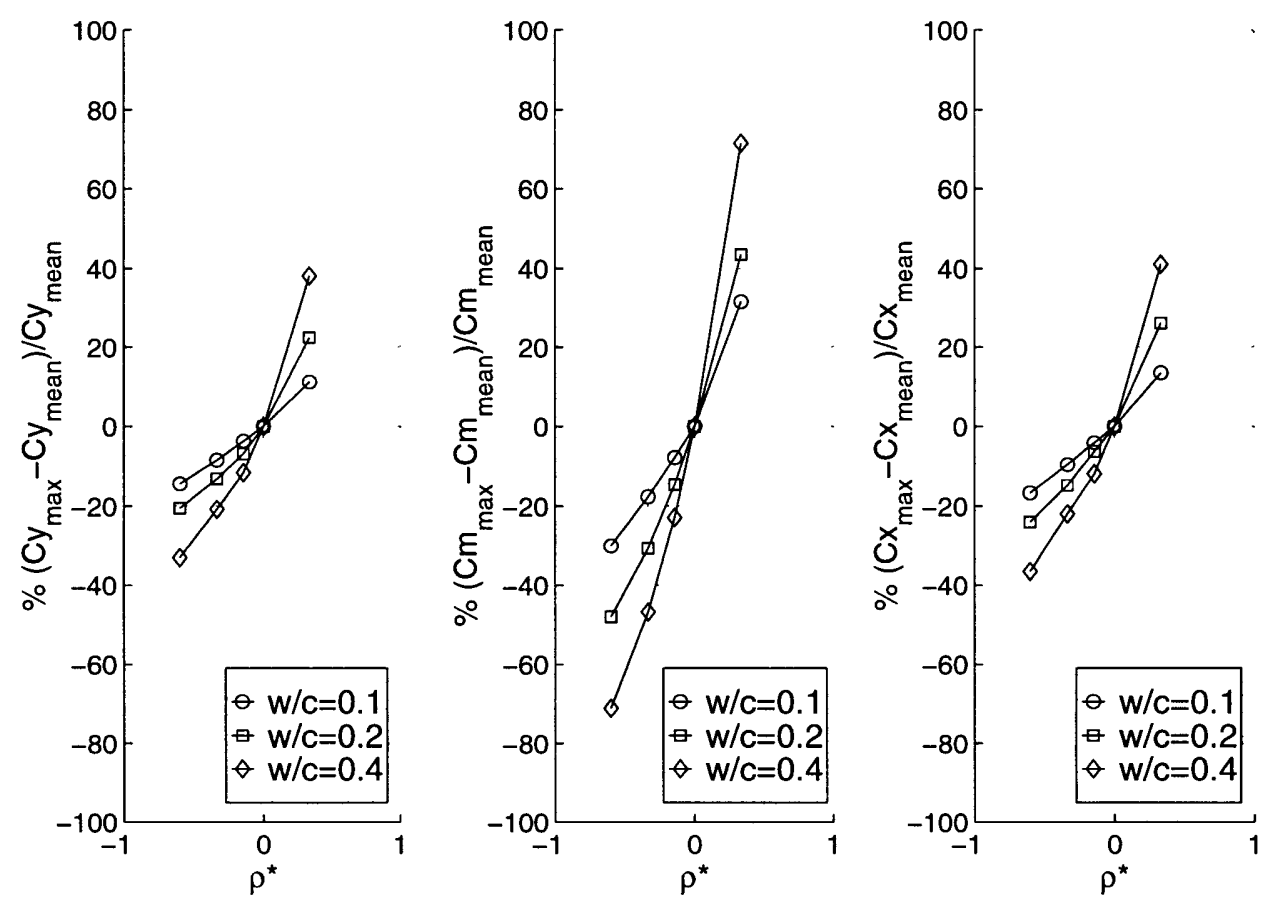

Figure 4-13: Maximum fluctuation in the force and moment coefficients as functions of density wake width and density parameter $\rho^{*}$. The straight lines joining data points are to help aid clarity. $M_{\infty}=0.63$.
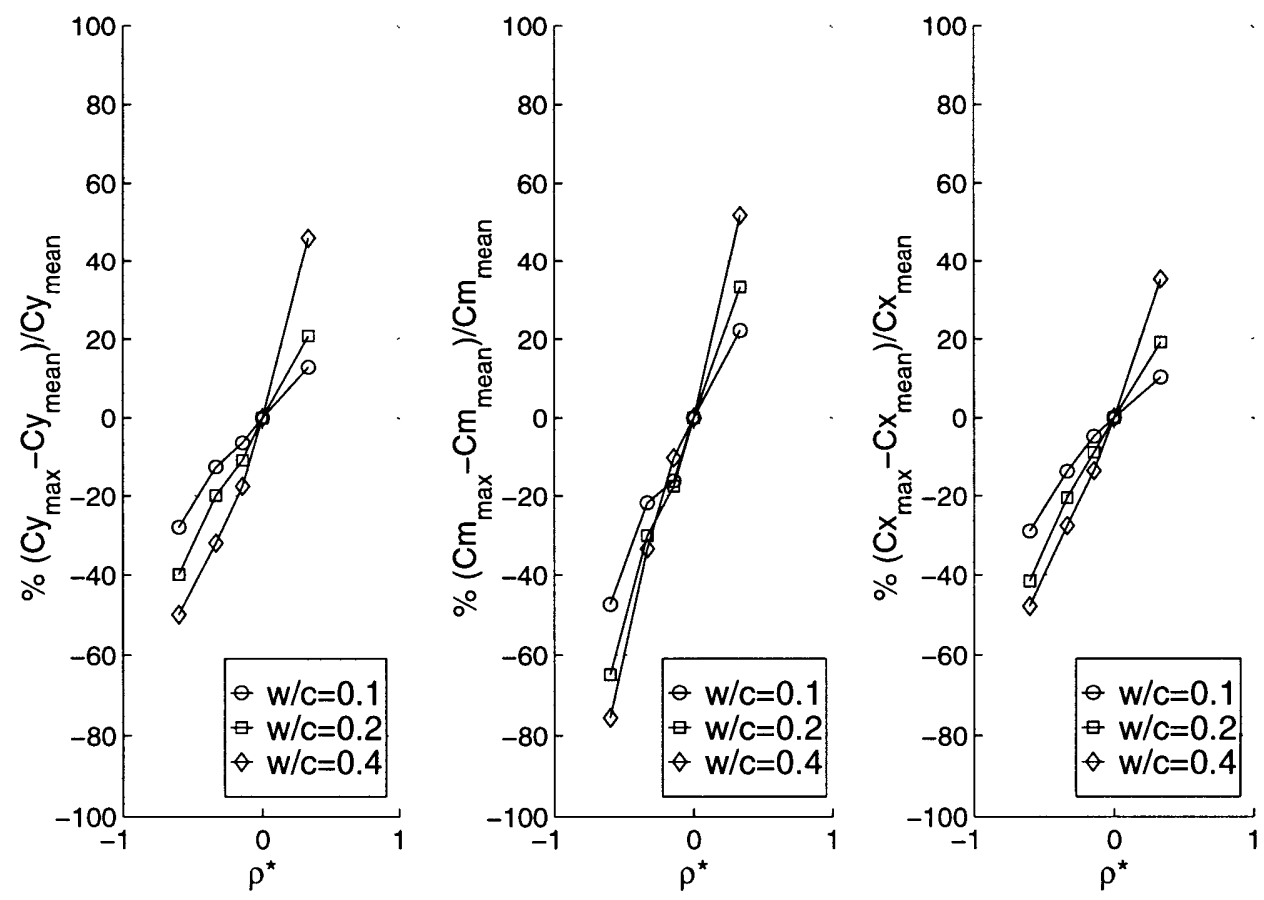

Figure 4-14: Maximum fluctuation in the force and moment coefficients as functions of density wake width and density parameter $\rho^{*}$. The straight lines joining data points are to help aid clarity. $M_{\infty}=0.87$. 


\begin{tabular}{|c|c|r|r|r|}
\hline \hline Coefficient & Wake Width (w/c) & $\Pi_{1}$ & $\Pi_{2}$ & $\Pi_{3}$ \\
\hline$C y$ & 0.1 & 1.9 & 23.0 & -0.1 \\
& 0.2 & 7.2 & 40.4 & 0.2 \\
& 0.4 & 28.7 & 73.4 & 0.2 \\
& 1.0 & 95.0 & 149.3 & 1.1 \\
\hline$C m$ & 0.1 & -4.4 & 41.7 & -0.5 \\
& 0.2 & 3.9 & 74.5 & -0.7 \\
& 0.4 & 60.6 & 143.0 & 0.9 \\
& 1.0 & 141.9 & 226.8 & 2.3 \\
\hline$C x$ & 0.1 & -2.7 & 28.7 & 0.0 \\
& 0.2 & 5.2 & 53.4 & 0.2 \\
& 0.4 & 40.6 & 101.7 & 0.7 \\
& 1.0 & 118.5 & 191.8 & 2.3 \\
\hline \hline
\end{tabular}

Table 4.4: Constants in the functional relationships for the maximum fluctuation in the force and moment coefficients (primary response). $M_{\infty}=0.15$.

$$
\begin{gathered}
\% \frac{C y_{\text {max }}-C y_{\text {mean }}}{C y_{\text {mean }}}=\Pi_{1} \rho^{* 2}+\Pi_{2} \rho^{*}+\Pi_{3} \\
\% \frac{C m_{\text {max }}-C m_{\text {mean }}}{C m_{\text {mean }}}=\Pi_{1} \rho^{* 2}+\Pi_{2} \rho^{*}+\Pi_{3} \\
{ }_{\%} \frac{C x_{\text {max }}-C x_{\text {mean }}}{C x_{\text {mean }}}=\Pi_{1} \rho^{* 2}+\Pi_{2} \rho^{*}+\Pi_{3}
\end{gathered}
$$

The constants $\Pi_{1}, \Pi_{2}$ and $\Pi_{3}$ in the functional relationships are tabulated in Table 4.4 through Table 4.7 for each free stream Mach number flow. Note the functional relationships obtained here represent "best fit" curves to the data in a least-squares sense. This results in a small non-zero value for the $\Pi_{3}$ constant, often 2 orders of magnitude smaller than the values for $\Pi_{1}$ and $\Pi_{2}$, which can be ignored.

The $\Pi_{2}$ constant for all force and moment coefficients is seen to increase by a factor of between 1.5-2.0 as the wake width doubles. The value for $\Pi_{1}$ also increases with increasing wake width, however in a much less defined manner by a factor of between 2-20 as the 


\begin{tabular}{|c|c|r|r|r|}
\hline \hline Coefficient & Wake Width (w/c) & $\Pi_{1}$ & $\Pi_{2}$ & $\Pi_{3}$ \\
\hline$C y$ & 0.1 & 8.7 & 28.4 & 0.5 \\
& 0.2 & 23.3 & 53.0 & -0.1 \\
& 0.4 & 49.9 & 87.5 & 1.0 \\
\hline$C m$ & 0.1 & 44.5 & 72.2 & -0.3 \\
& 0.2 & 104.2 & 127.0 & 1.0 \\
& 0.4 & 87.3 & 157.5 & 0.4 \\
\hline$C x$ & 0.1 & 18.5 & 35.8 & 1.4 \\
& 0.2 & 33.8 & 60.8 & 1.4 \\
& 0.4 & 54.8 & 97.3 & 2.0 \\
\hline \hline
\end{tabular}

Table 4.5: Constants in the functional relationships for the maximum fluctuation in the force and moment coefficients (primary response). $M_{\infty}=0.53$.

\begin{tabular}{|c|c|r|r|r|}
\hline \hline Coefficient & Wake Width (w/c) & $\Pi_{1}$ & $\Pi_{2}$ & $\Pi_{3}$ \\
\hline$C y$ & 0.1 & 9.0 & 29.7 & 0.3 \\
& 0.2 & 33.2 & 54.3 & 0.4 \\
& 0.4 & 58.9 & 90.7 & 0.9 \\
\hline$C m$ & 0.1 & 39.0 & 75.3 & 1.7 \\
& 0.2 & 52.4 & 111.7 & 0.3 \\
& 0.4 & 99.1 & 178.5 & 0.7 \\
\hline$C x$ & 0.1 & 10.9 & 35.0 & 0.5 \\
& 0.2 & 34.1 & 62.0 & 1.3 \\
& 0.4 & 59.2 & 97.3 & 1.4 \\
\hline \hline
\end{tabular}

Table 4.6: Constants in the functional relationships for the maximum fluctuation in the force and moment coefficients (primary response). $M_{\infty}=0.63$.

\begin{tabular}{|c|c|r|r|r|}
\hline \hline Coefficient & Wake Width (w/c) & $\Pi_{1}$ & $\Pi_{2}$ & $\Pi_{3}$ \\
\hline$C y$ & 0.1 & -10.6 & 40.2 & 0.4 \\
& 0.2 & -3.2 & 63.5 & -0.2 \\
& 0.4 & 62.0 & 118.9 & -0.5 \\
\hline$C m$ & 0.1 & -5.2 & 71.7 & -1.3 \\
& 0.2 & -9.6 & 100.6 & 0.2 \\
& 0.4 & -2.1 & 131.9 & 6.3 \\
\hline$C x$ & 0.1 & -21.3 & 36.2 & 0.5 \\
& 0.2 & -14.7 & 60.7 & 0.4 \\
& 0.4 & 26.9 & 95.6 & 0.2 \\
\hline \hline
\end{tabular}

Table 4.7: Constants in the functional relationships for the maximum fluctuation in the force and moment coefficients (primary response). $M_{\infty}=0.87$. 
wake width doubles. The largest increase in $\Pi_{1}$ is seen between wake widths of $0.2 c$ and 0.4c. The functional relations for $M_{\infty}=0.87$ have a relatively smaller magnitude for $\Pi_{1}$ compared to the lower Mach number functional relations. This is due to the near-linear variation in the the shock wave motion (and hence blade static pressure fluctuations) with increasing $\left|\rho^{*}\right|$ alluded to in the previous section.

The functional relations should be used with caution. In particular for density parameters $\rho^{*} \approx 0$ the $\Pi_{3}$ constant may become dominant and the deviation from the data can be large.

\section{Compressibility Scaling}

Inspection of Figure 4-11 through Figure 4-12 shows the force and moment fluctuation magnitudes to increase with flow Mach number. To determine an initial estimate for the effect of compressibility on the maximum force and moment fluctuations the Prandtl-Glauert scaling rule is used. This scaling rule is based on the linearized perturbation velocity potential equation and can be used to relate the pressure coefficient $C p$ at free stream Mach number $M_{\infty}$ to the pressure coefficient $C p_{o}$ at Mach number $M_{\infty}=0.0$ as follows [1],

$$
C p=\frac{C p_{o}}{\sqrt{1-M_{\infty}^{2}}}
$$

The incompressible and compressible force and moment coefficients can also be related in the same way. To asses the applicability of this relation with regards to density wake induced force and moment fluctuations the maximum fluctuations at each free stream Mach number are multiplied by the Prandtl-Glauert compressibility factor $\sqrt{1-M_{\infty}^{2}}$ i.e., 


$$
\begin{gathered}
C y_{o}(\max )=C y(\max ) \times \sqrt{1-M_{\infty}^{2}} \\
C x_{o}(\max )=C x(\max ) \times \sqrt{1-M_{\infty}^{2}} \\
C m_{o}(\max )=C m(\max ) \times \sqrt{1-M_{\infty}^{2}}
\end{gathered}
$$

and compared in Figure C-1 through Figure C-12 in Appendix C. The following conclusions can be made from these plots.

- For wake widths of $0.1 c$ the maximum fluctuation in the azimuthal force coefficient for all compressible tests compares to within $5 \%$ of the corresponding incompressible inviscid result. For wake widths of $0.2 c$ the deviations from the incompressible result increase from $5 \%$ to a maximum of $20 \%$ for $\left|\rho^{*}\right| \geq 1 / 3$. Similarly for wake widths of $0.4 c$ deviations of up to $40 \%$ can be seen for $\left|\rho^{*}\right| \geq 1 / 3$.

- For wake widths of $0.1 c$ the maximum fluctuation in the axial force coefficient for $M_{\infty}=0.53, M_{\infty}=0.63$ and $M_{\infty}=0.87$ deviates up to a maximum of $35 \%$ from the corresponding result at $M_{\infty}=0.15$ for all $\rho^{*}$. For wake widths of $0.2 c$ and $0.4 c$ a deviation of more than $50 \%$ can be seen between the compressible results.

- For wake widths of $0.1 c$ and $0.2 c$ the maximum fluctuation in the moment coefficient deviates up to $35 \%$ between the $M_{\infty}=0.15$ and the incompressible calculation. For wake widths of $0.1 c$ there is a maximum deviation of $60 \%$ for $\rho^{*}<0$ and a maximum deviation of $100 \%$ for $\rho^{*}>0$. For larger wake widths the deviations exceed $100 \%$.

Clearly the Prandtl-Glauert compressibility factor is not adequate to describe the free stream Mach number effects on the maximum fluctuation in the force and moment coefficients. Reasonable scaling for the azimuthal force coefficient can be achieved however but only for small wake widths $(w / c=0.1)$. 


\subsection{Secondary Response}

The viscous compressible results indicate continued force and moment fluctuations after the density wake leaves the cascade blade passage (Figure 4-1). The amplitude of these fluctuations are typically 2-10 times greater than the amplitude of the vortex shedding induced fluctuations in the baseline flow. Similar to the analysis for the primary response the source of the secondary response fluctuations are identified and a parametric study is conducted to establish trends for the variation of the maximum amplitudes with varying density wake properties.

\subsubsection{General Flow Features}

The source of the continued fluctuation in the secondary response can be identified by inspection of the flow field Mach number contour plots in Figure 4-15 through 4-18. These plots indicate the deformation of the blade suction surface boundary layer during the passage of a density wake of width $0.2 c$ and density ratio 0.5 . A local decrease in the boundary layer thickness can be seen immediately below the density wake as the fluid flux associated with the counterrotating vortex pairs impinges on the blade suction surface. As the fluid flux spreads upstream the boundary layer fluid is decelerated and an increase in the boundary layer thickness can be seen. As the density wake leaves the blade passage the decelerated boundary layer fluid re-accelerates and forms a separation bubble on the blade suction surface. The separation bubble consists of a concentrated flow re-circulation zone with a low pressure core region. In addition a portion of the density wake fluid remains "trapped" inside the bubble removed from the main body of the density wake ${ }^{3}$ (see Figure 4-5).

As the separation bubble convects downstream, so does the flow separation and reattachment points on the blade suction surface as illustrated in Figure 4-19. Initially the separation point is located at $0.94 c$. As the separation bubble is formed the boundary layer separation point advances rapidly upstream to $0.76 c$ and is found to re-attach at $0.84 c$.

\footnotetext{
${ }^{3}$ This trapped low density fluid corresponds to a high temperature "spot" on the blade surface. This may influence the blade thermal stress distribution.
} 


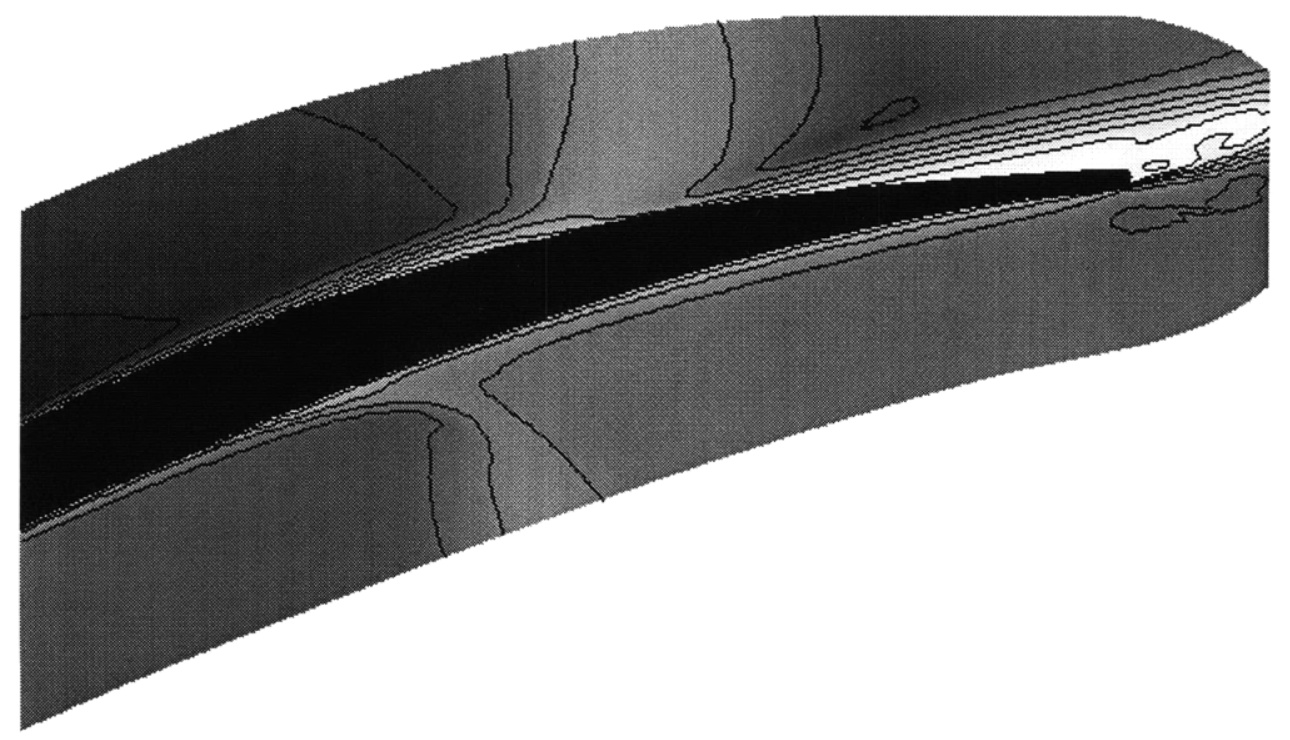

Figure 4-15: Mach number contour image showing boundary layer deformation during passage of a density wake of width $0.2 c$ and density ratio $0.5 . M_{\infty}=0.15 . \tau=0.78$.

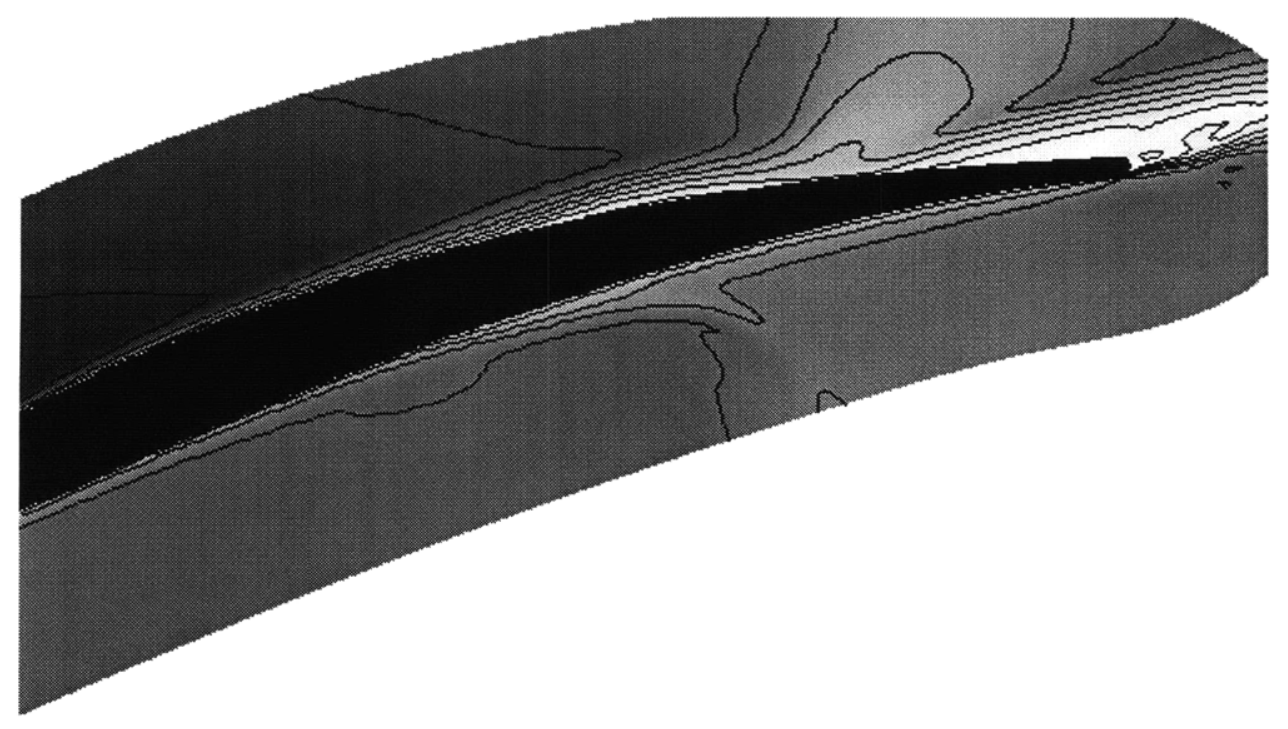

Figure 4-16: Mach number contour image showing boundary layer deformation during passage of a density wake of width $0.2 c$ and density ratio $0.5 . M_{\infty}=0.15 . \tau=1.03$. 


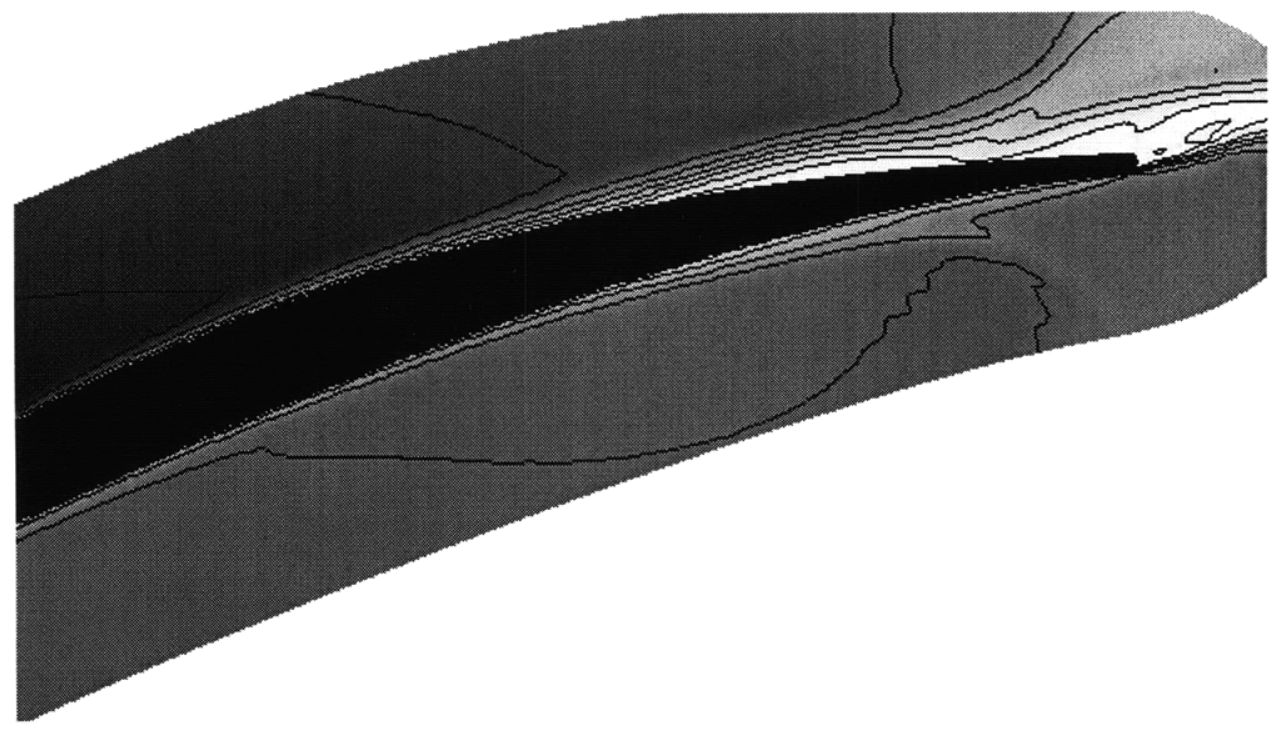

Figure 4-17: Mach number contour image showing boundary layer deformation during passage of a density wake of width $0.2 c$ and density ratio $0.5 . M_{\infty}=0.15 . \tau=1.28$.

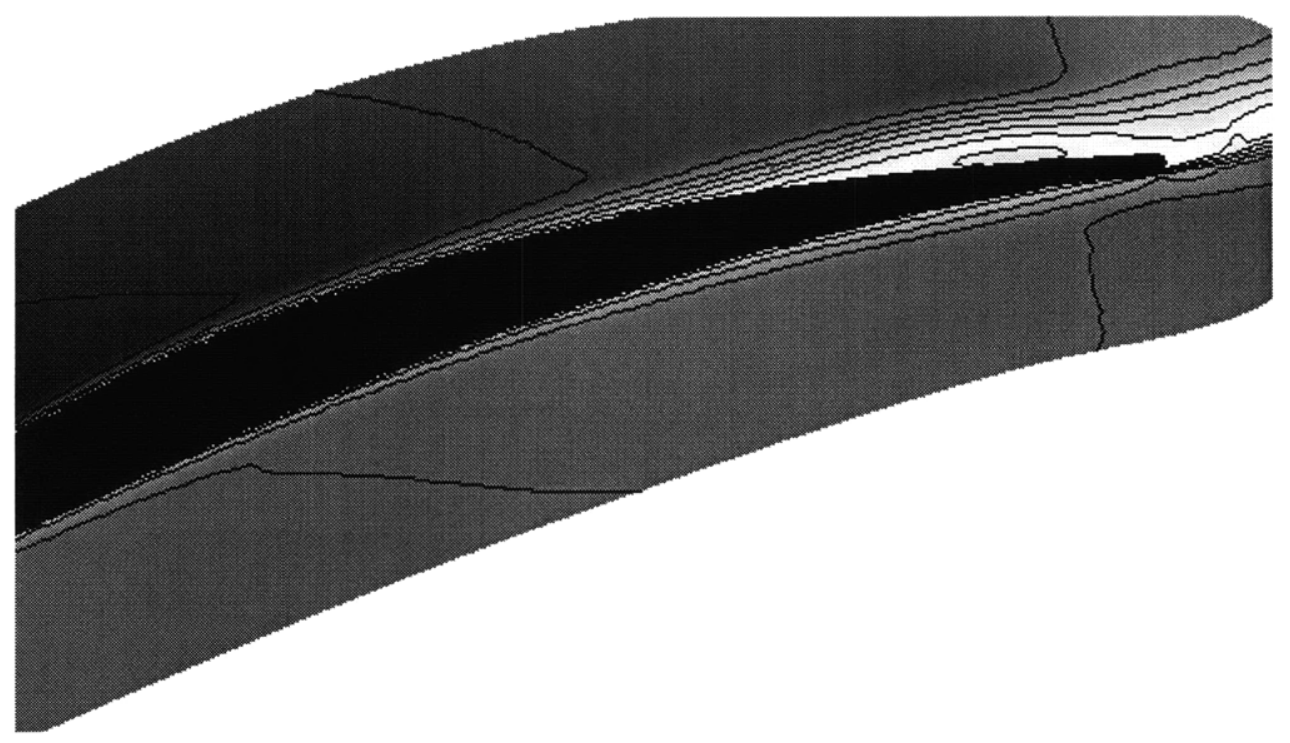

Figure 4-18: Mach number contour image showing boundary layer deformation during passage of a density wake of width $0.2 c$ and density ratio $0.5 . M_{\infty}=0.15 . \tau=1.53$. 
The separation bubble is then seen to grow rapidly in the stream wise direction to a maximum length of almost $0.20 c$. After the separation bubble leaves the blade suction surface continued fluctuation in the separation point can be seen with amplitude $0.05 c$.

The separation bubble also disrupts the periodic vortex shedding at the blade trailing edge. This can be seen in the vorticity contour image in Figure 4-20 taken downstream of the blade trailing edge for flow at $M_{\infty}=0.87$. Similar suppression of vortex shedding is seen at all other Mach number flows. For the case shown in Figure 4-20 the regular vortex shedding pattern is not recovered until approximately $2-3$ convective time units have passed after the density wake leaves the blade trailing edge.

The suppression of vortex shedding removes the vortex shedding frequency in the force and moment fluctuations. The frequency content of the baseline fluctuations in the $M_{\infty}=0.87$ flow shown in Figure 3-13 can be compared with the secondary response frequency content calculated between $\tau=2.0$ and $\tau=6.0$ shown in Figure 4-21. Note the absence of the dominant peaks at $\mu=1.9$ in the secondary response frequency spectrum. This clearly indicates the absence of vortex shedding in the downstream flow. This can be verified in all the parametric results by simple examination of the time histories of the secondary response force and moment fluctuations shown in Figure B-1 through Figure B-52 in Appendix B). The secondary response of all the force and moment fluctuations have a distinct lack of high frequency content compared to the primary response.

The amplitude of the secondary response fluctuations is found to increase with the increase in upstream motion of the blade separation point. This is examined further in the next section.

\subsubsection{Parametric Study}

The maximum upstream motion of the suction surface separation point is plotted in Figure 4-22 through Figure 4-25 for varying density wake widths and density ratios for each free 


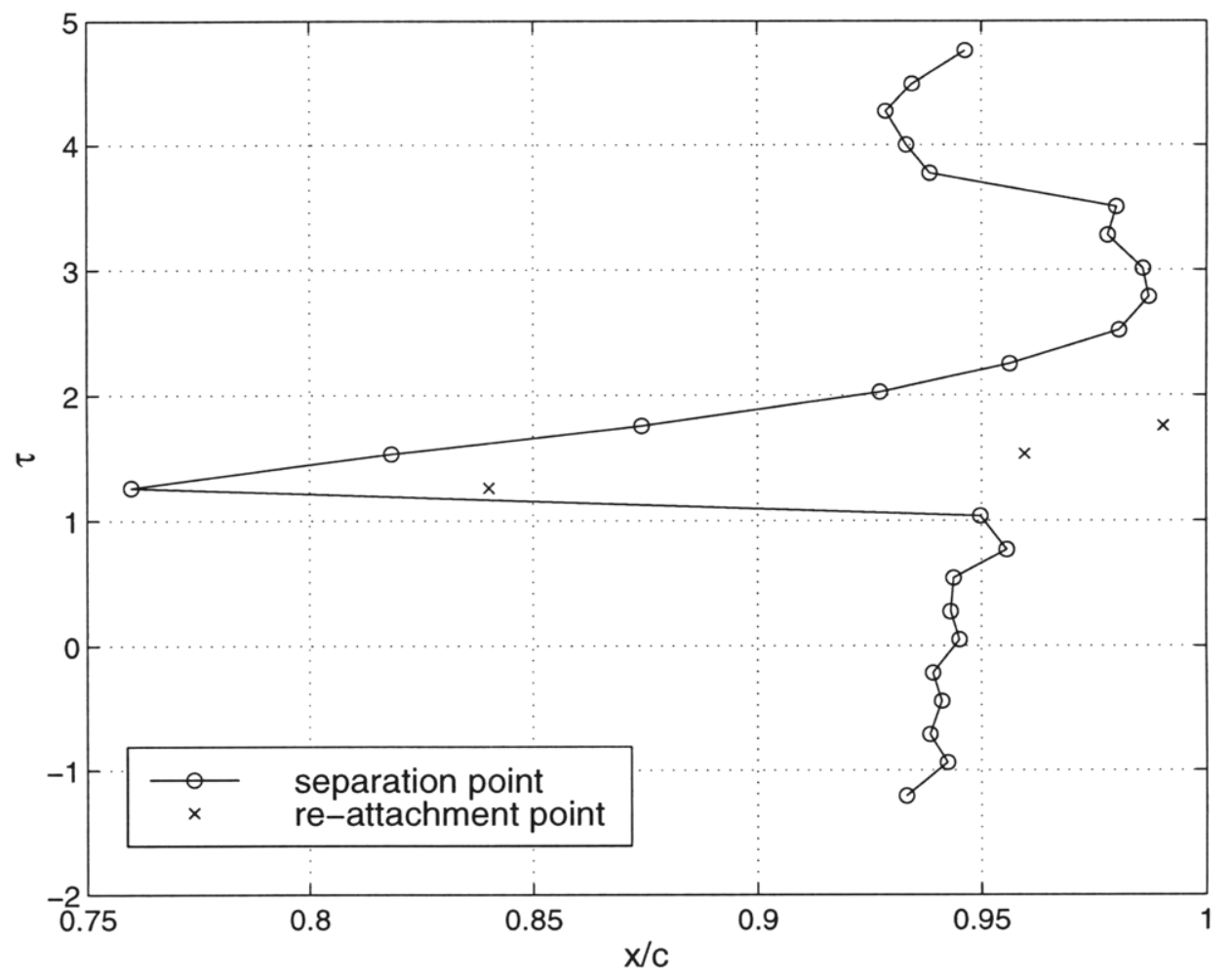

Figure 4-19: The fluctuation of the flow separation and re-attachment points of the suction surface boundary layer during passage of a density wake of width $0.2 c$ and density ratio $0.5 . M_{\infty}=0.15$.

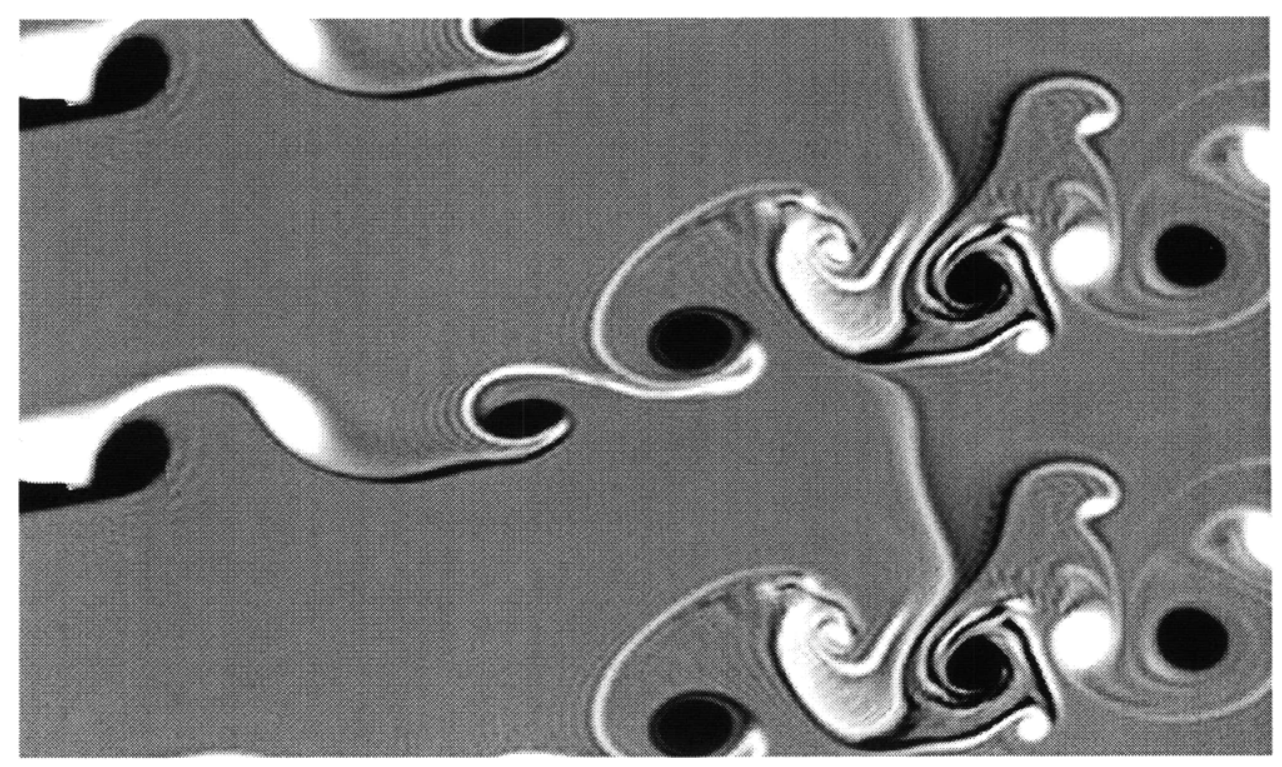

Figure 4-20: Vorticity contour image showing the disruption of regular vortex shedding behind the blade trailing edge. The density wake is located $1.0 c$ downstream of the trailing edge entrained inside the vortex wake. $\tau=3.1, w / c=0.2, \rho_{2} / \rho_{1}=0.5, M_{\infty}=0.87$. 

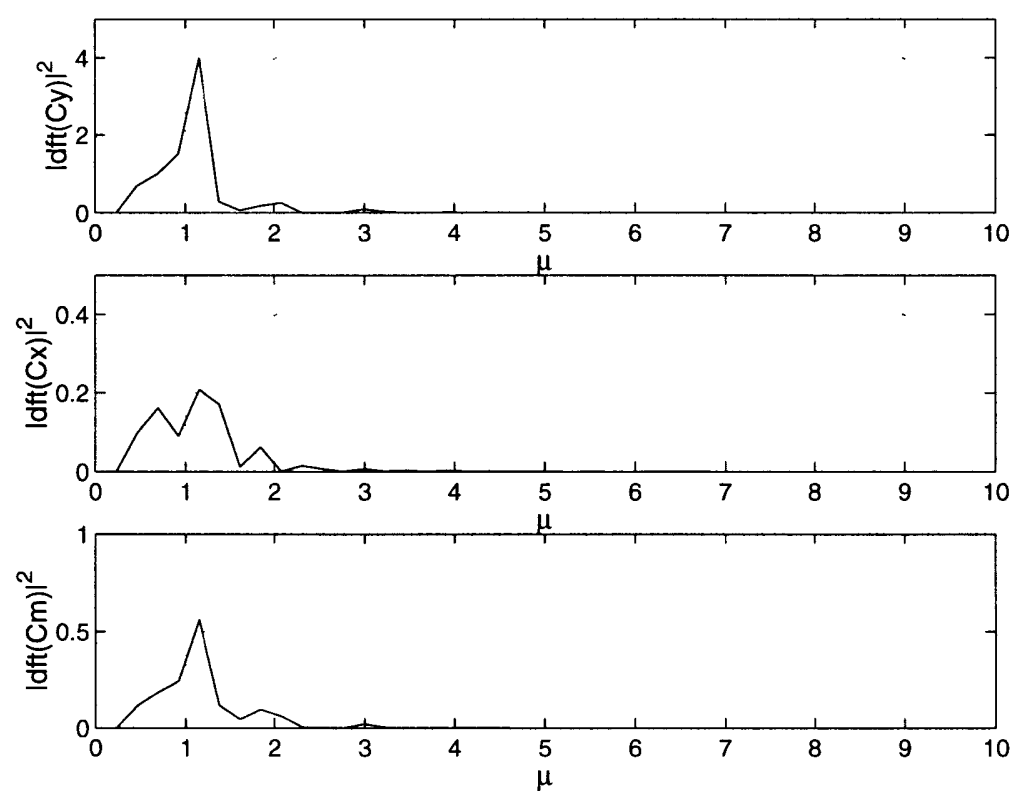

Figure 4-21: Discrete fourier transform of the secondary response force and moment coefficient fluctuations. $w / c=0.2, \rho_{2} / \rho_{1}=0.5, M_{\infty}=0.87 . d f t(X)$ is the discrete fourier transform of the time signal $X . \mu=$ non-dimensional frequency.

stream Mach number flow. In general the separation point moves further upstream as the wake density ratio decreases (for a high density wake $\rho_{2} / \rho_{1}>1$ the separation point moves further upstream as the density ratio increases). An increase in density wake width also drives the separation point further upstream however there appears to be an upper limit $(w / c=0.2)$ beyond which further increase in density wake width does not further affect the separation point motion. This is particularly evident in the $M_{\infty}=0.15, M_{\infty}=0.53$ and $M_{\infty}=0.63$ results for density ratios less than 1.0 .

The corresponding maximum fluctuation in the force and moment coefficients for the secondary response are plotted in Figure 4-26 through Figure 4-29. The maximum amplitude of the secondary response is normalized by the amplitude of the baseline fluctuations. The fluctuation amplitudes follow the same trends as the separation point fluctuation. Note how the curves for wake widths of $0.2 c$ and $0.4 c$ almost collapse together. This corresponds to the same trend observed for the separation point fluctuation in Figure 4-22 through Figure 4-25. The following conclusions can be made from the trends observed for the secondary response amplitudes: 


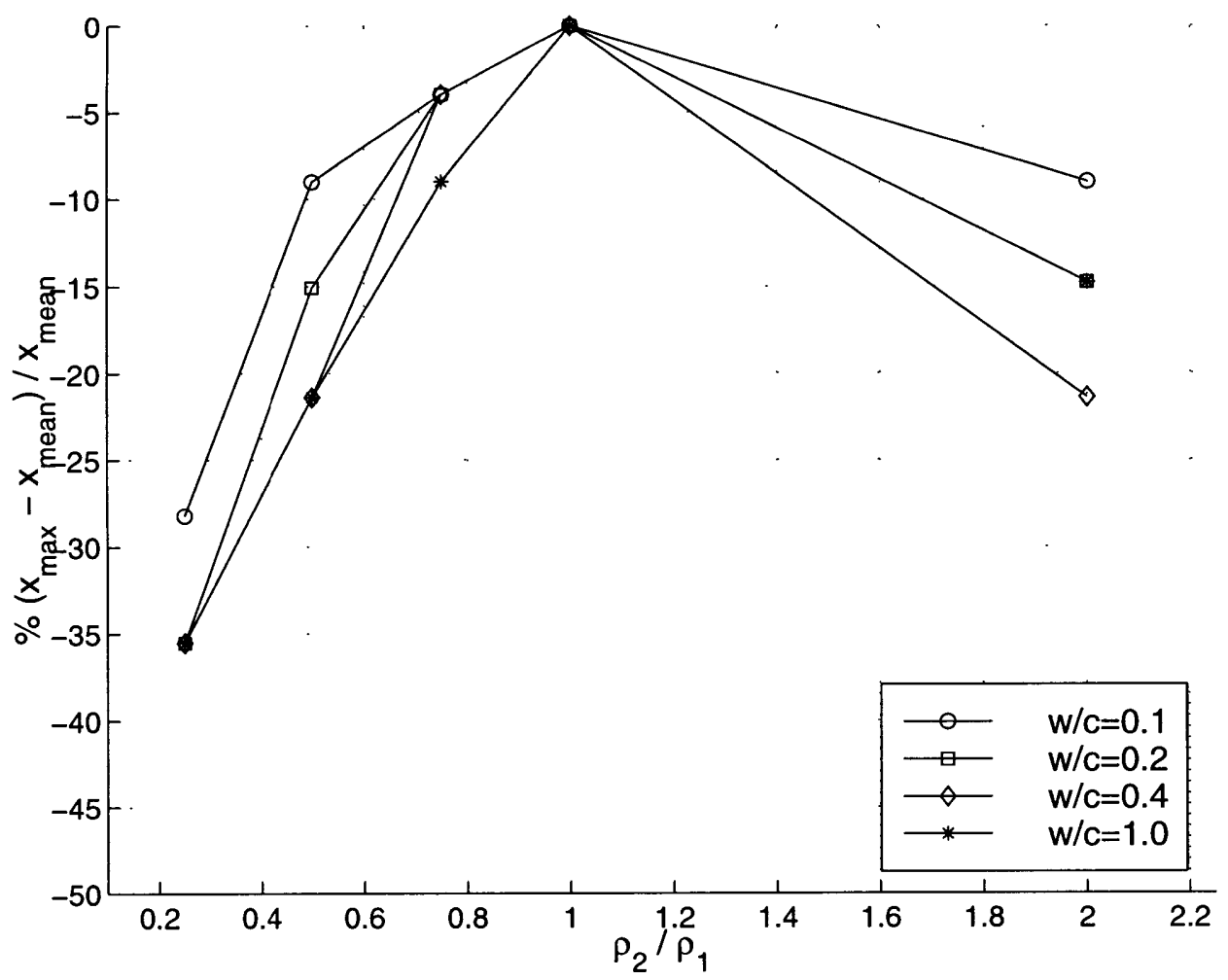

Figure 4-22: The maximum change in the suction surface separation point from the mean baseline position as a function of wake width and density ratio. $M_{\infty}=0.15$

- The maximum upstream motion of the separation point depends solely on the density ratio for wake widths greater than $0.2 c$ and for density ratios $\rho_{2} / \rho_{1}<1.0$. The corresponding amplitude of the secondary response is similarly bounded by the wake width for density ratios $\rho_{2} / \rho_{1}<1.0$. This shows a clear link between the separation point motion and the amplitude of the secondary response fluctuations.

- The amplitude of the secondary fluctuations normalized by the baseline fluctuation amplitudes are seen to decrease with increasing free stream Mach number. This is a direct consequence of the increased amplitude of the baseline fluctuations however (see Table 3.2). In general the secondary fluctuations for all coefficients (without normalization) increases with Mach number.

\section{Functional Relationships}

Functional relationships can be derived to help quantify the trends in the secondary response. The change in the amplitude of the force and moment coefficients with density 


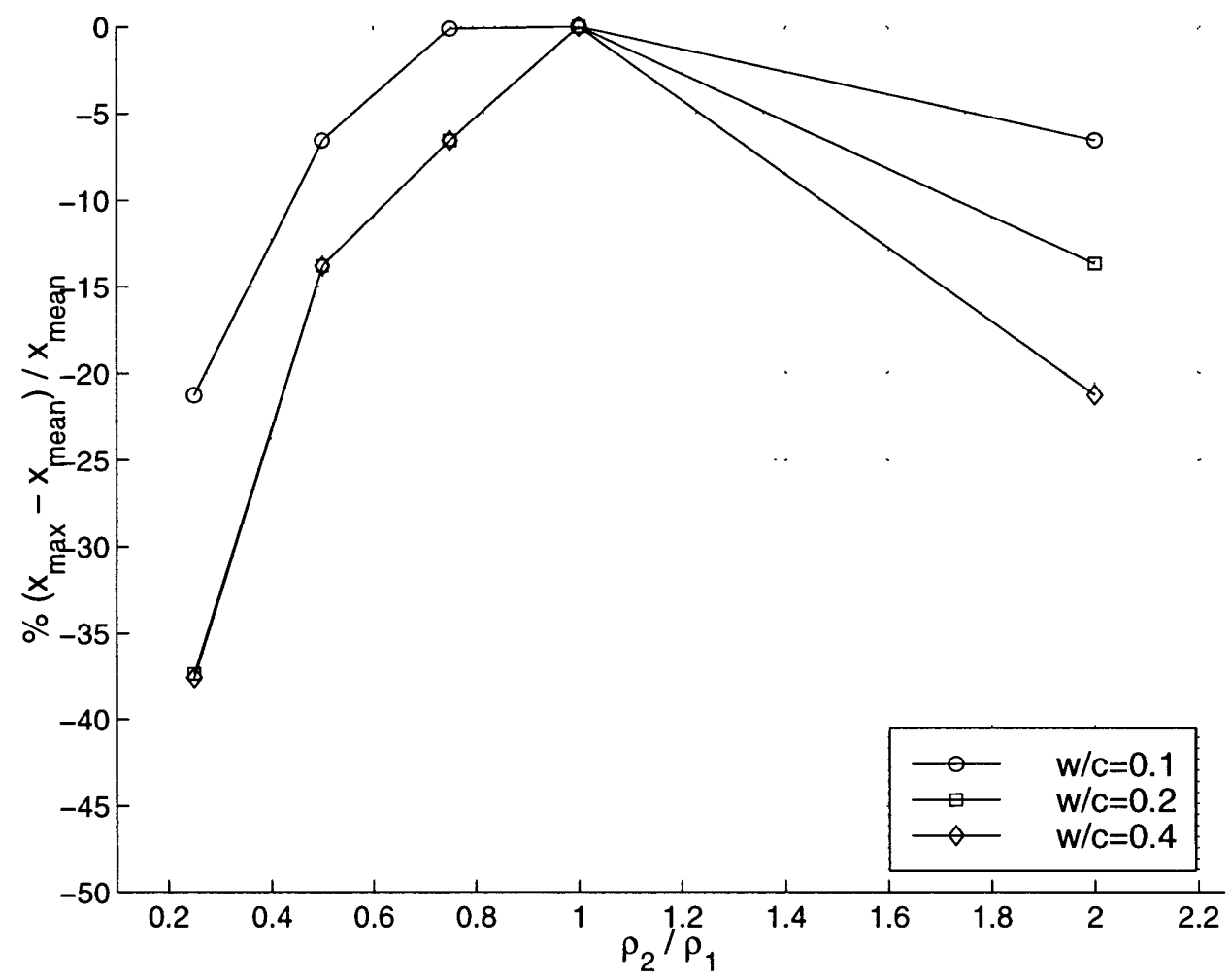

Figure 4-23: The maximum change in the suction surface separation point from the mean baseline position as a function of wake width and density ratio. $M_{\infty}=0.53$.

ratio $\rho_{2} / \rho_{1}$ can be expressed by the following algebraic relationships,

$$
\begin{gathered}
\% \frac{C y_{\text {second }}}{C y_{\text {baseline }}}=\Phi_{1}\left(\rho_{2} / \rho_{1}\right)^{2}+\Phi_{2}\left(\rho_{2} / \rho_{1}\right)+\Phi_{3} \\
\% \frac{C m_{\text {second }}}{C m_{\text {baseline }}}=\Phi_{1}\left(\rho_{2} / \rho_{1}\right)^{2}+\Phi_{2}\left(\rho_{2} / \rho_{1}\right)+\Phi_{3} \\
\% \frac{C x_{\text {second }}}{C x_{\text {baseline }}}=\Phi_{1}\left(\rho_{2} / \rho_{1}\right)^{2}+\Phi_{2}\left(\rho_{2} / \rho_{1}\right)+\Phi_{3}
\end{gathered}
$$

The constants $\Phi_{1}, \Phi_{2}$ and $\Phi_{3}$ in the functional relationships are tabulated in Table 4.8 through Table 4.11 for each free stream Mach number flow. Note the functional relationships obtained here represent "best fit" curves to the data in a least-squares sense and are valid only for $\rho_{2} / \rho_{1}<1.0$. Insufficient data points are available to obtain good curve fits 


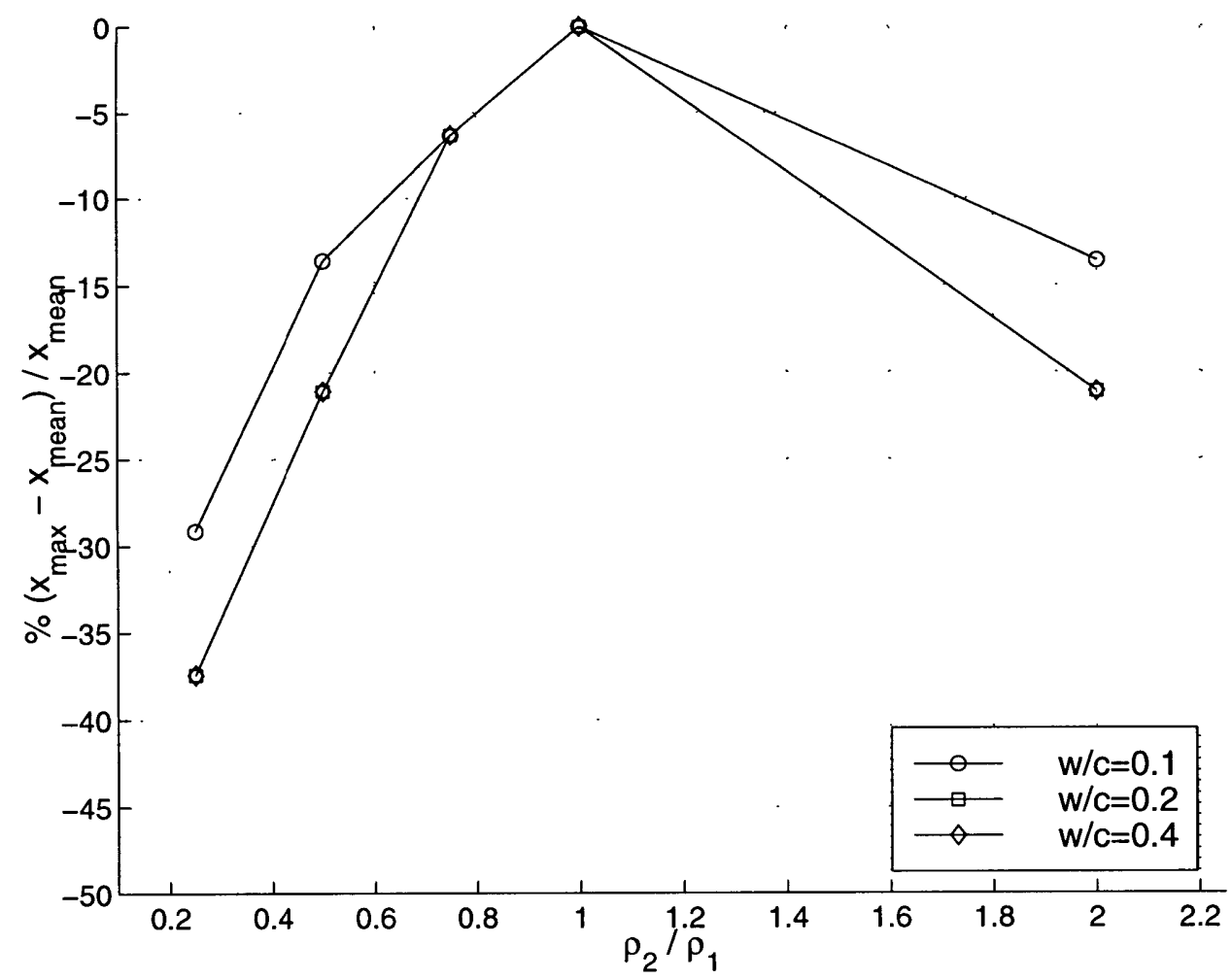

Figure 4-24: The maximum change in the suction surface separation point from the mean baseline position as a function of wake width and density ratio. $M_{\infty}=0.63$.

for $\rho_{2} / \rho_{1}>1.0$.

\subsubsection{Summary}

The force and moment fluctuations induced by passage of density wakes in viscous compressible flows have been characterized for flow Mach numbers ranging from $M_{\infty}=0.15$ to $M_{\infty}=0.87$ and for flow Reynolds number $\operatorname{Re}\left(c, U_{\infty}\right) \approx 700,000$. The controlling flow features responsible for the force and moment fluctuations have been identified and quantified. The following is a summary of the key results.

- The primary response force and moment fluctuations for the $M_{\infty}=0.15,0.53$ and 0.63 flows are characterized by the magnitude of the fluid flux directed to the blade surfaces (similar to the inviscid incompressible flow force and moment fluctuations). For flows with shock waves however $\left(M_{\infty}=0.87\right)$ the maximum force and moment 


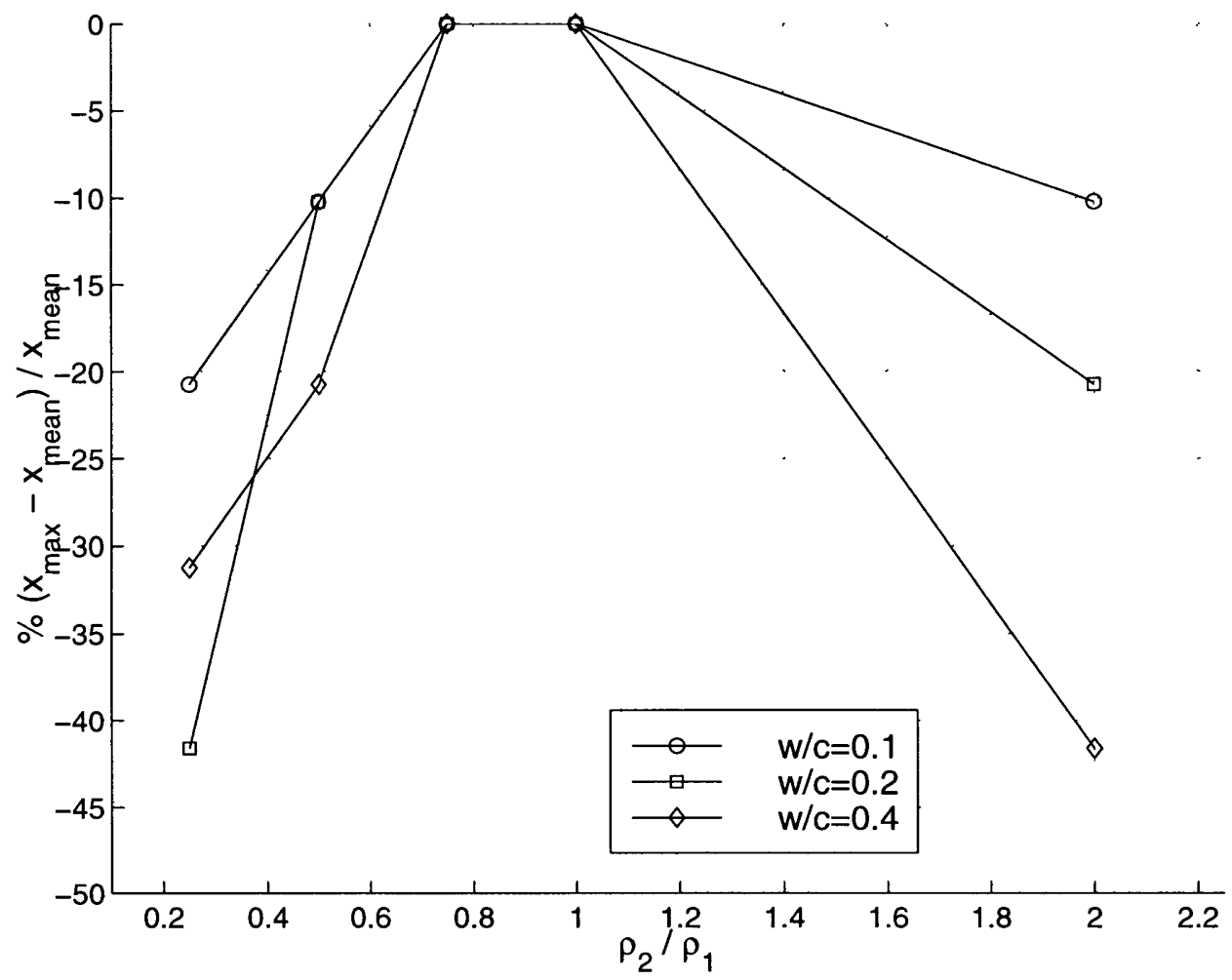

Figure 4-25: The maximum change in the suction surface separation point from the mean baseline position as a function of wake width and density ratio. $M_{\infty}=0.87$.
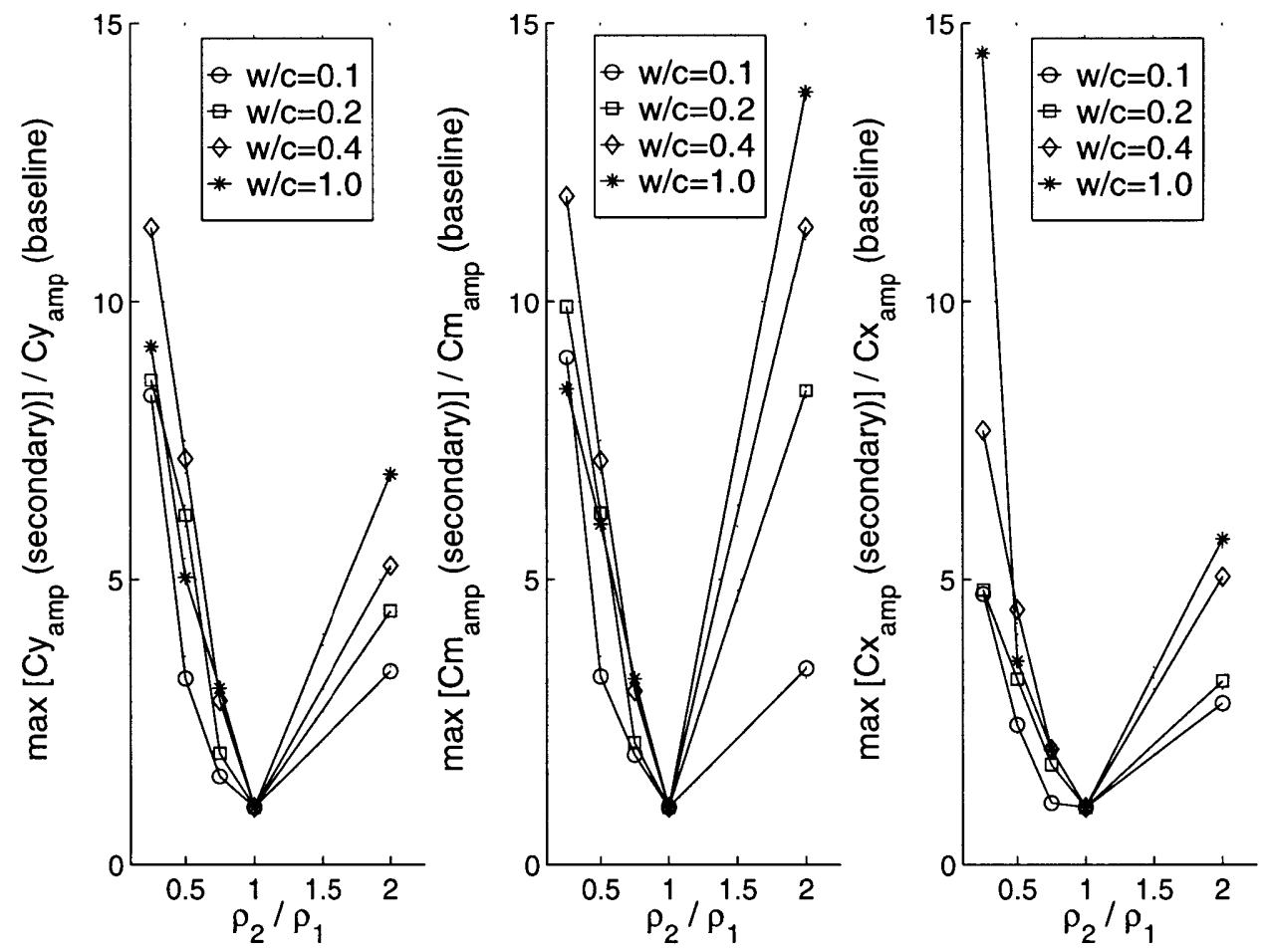

Figure 4-26: The maximum fluctuation in the blade azimuthal force, axial force and moment coefficients in the secondary response region for varying density wake widths and density ratios. $M_{\infty}=0.15$. 

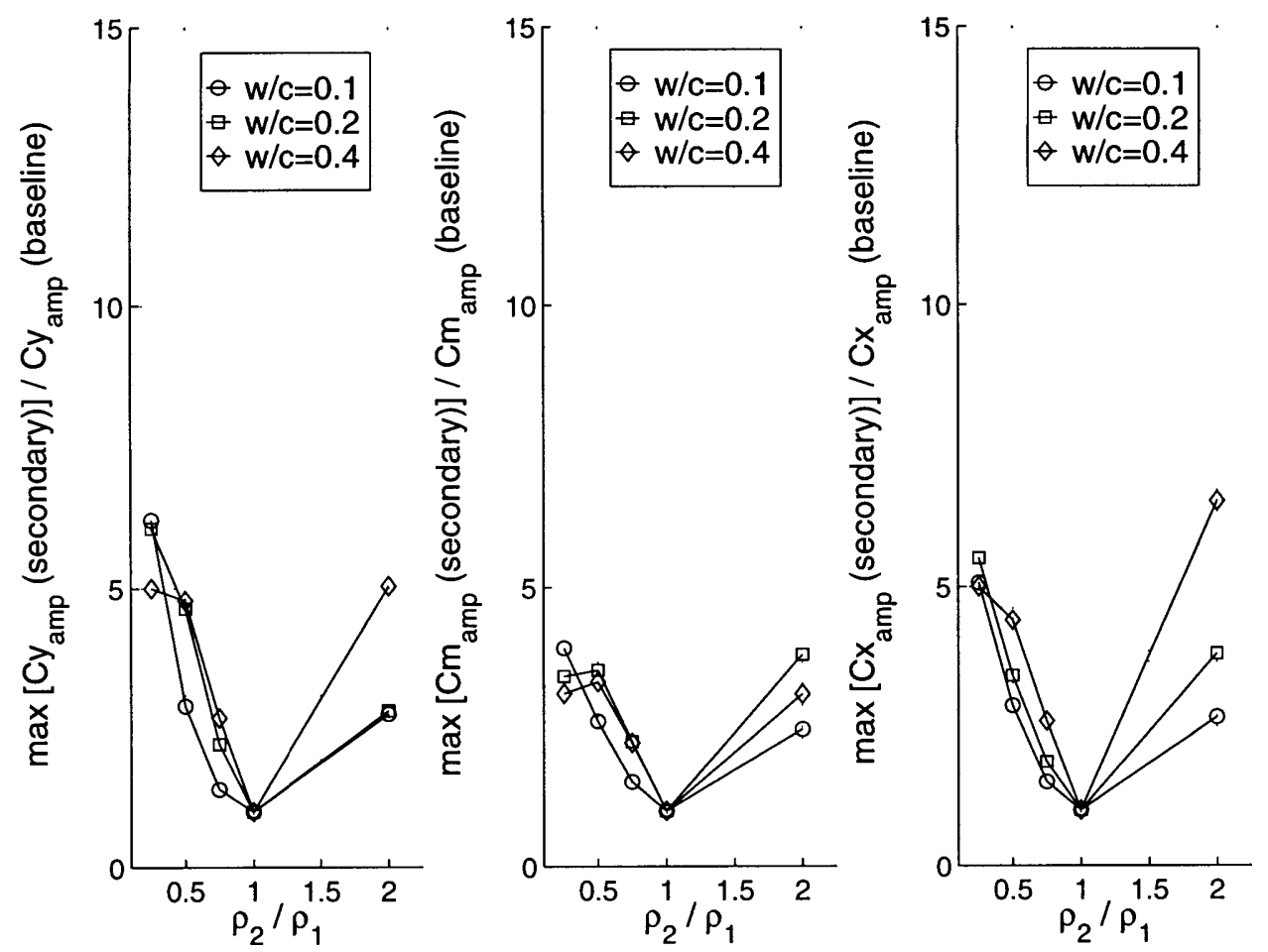

Figure 4-27: The maximum fluctuation in the blade azimuthal force, axial force and moment coefficients in the secondary response region for varying density wake widths and density ratios. $M_{\infty}=0.53$.
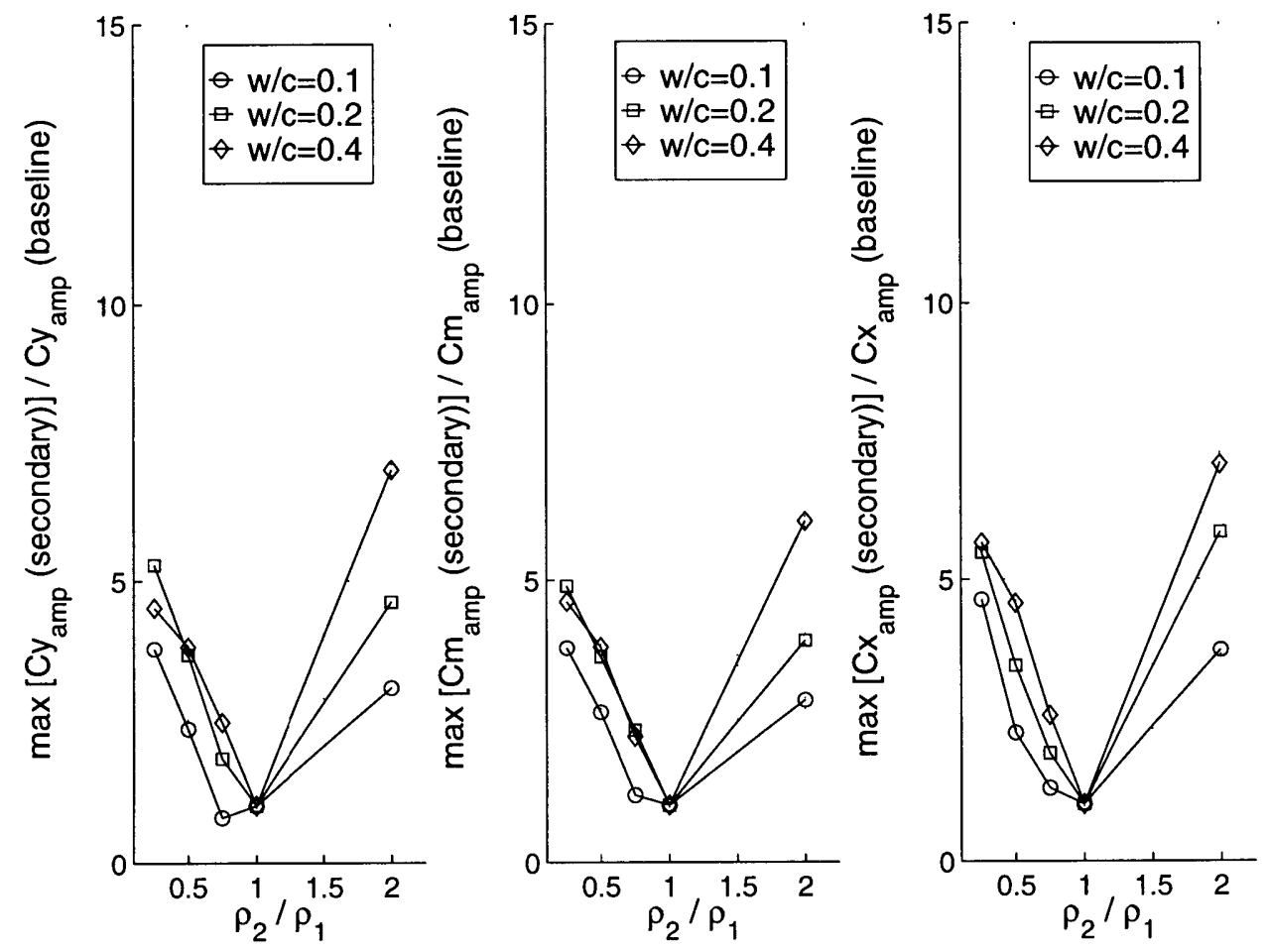

Figure 4-28: The maximum fluctuation in the blade azimuthal force, axial force and moment coefficients in the secondary response region for varying density wake widths and density ratios. $M_{\infty}=0.63$. 

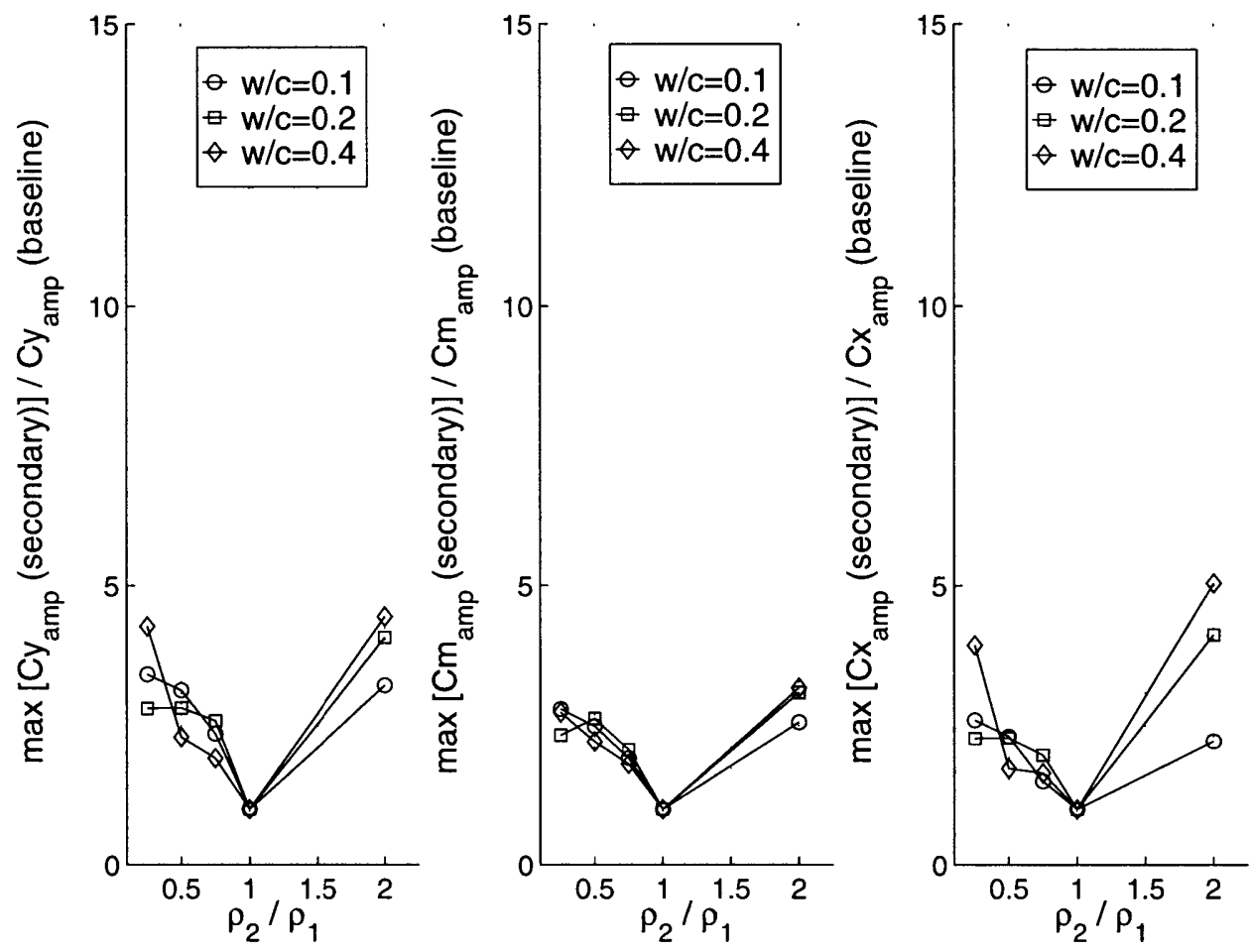

Figure 4-29: The maximum fluctuation in the blade azimuthal force, axial force and moment coefficients in the secondary response region for varying density wake widths and density ratios. $M_{\infty}=0.87$.

\begin{tabular}{|c|c|r|r|r|}
\hline \hline Coefficient & Wake Width (w/c) & $\Phi_{1}$ & $\Phi_{2}$ & $\Phi_{3}$ \\
\hline$C y$ & 0.1 & 18.0 & -32.0 & 15.1 \\
& 0.2 & 5.9 & -18.1 & 13.0 \\
& 0.4 & 9.2 & -25.7 & 17.3 \\
& 1.0 & 8.2 & -20.9 & 13.8 \\
\hline$C m$ & 0.1 & 19.0 & -34.0 & 16.1 \\
& 0.2 & 10.3 & -25.2 & 15.7 \\
& 0.4 & 10.9 & -28.4 & 18.4 \\
& 1.0 & 0.7 & -10.8 & 11.1 \\
\hline$C x$ & 0.1 & 8.8 & -16.1 & 8.2 \\
& 0.2 & 3.2 & -9.2 & 7.0 \\
& 0.4 & 8.7 & -19.9 & 12.1 \\
& 1.0 & 39.5 & -66.1 & 28.1 \\
\hline \hline
\end{tabular}

Table 4.8: Constants in the functional relationships for the maximum fluctuation in the force and moment coefficients (secondary response). Valid for $\rho_{2} / \rho_{1}<1.0 . M_{\infty}=0.15$. 


\begin{tabular}{|c|c|r|r|r|}
\hline \hline Coefficient & Wake Width (w/c) & $\Phi_{1}$ & $\Phi_{2}$ & $\Phi_{3}$ \\
\hline$C y$ & 0.1 & 11.7 & -21.5 & 10.8 \\
& 0.2 & 0.9 & -8.1 & 8.2 \\
& 0.4 & -5.8 & 1.7 & 5.1 \\
\hline$C m$ & 0.1 & 3.2 & -7.9 & 5.7 \\
& 0.2 & -5.4 & 3.4 & 3.0 \\
& 0.4 & -5.7 & 4.2 & 2.5 \\
\hline$C x$ & 0.1 & 6.8 & -13.9 & 8.1 \\
& 0.2 & 5.0 & -12.3 & 8.3 \\
& 0.4 & -4.0 & -0.5 & 5.5 \\
\hline \hline
\end{tabular}

Table 4.9: Constants in the functional relationships for the maximum fluctuation in the force and moment coefficients (secondary response). Valid for $\rho_{2} / \rho_{1}<1.0 . M_{\infty}=0.53$.

\begin{tabular}{|c|c|r|r|r|}
\hline \hline Coefficient & Wake Width (w/c) & $\Phi_{1}$ & $\Phi_{2}$ & $\Phi_{3}$ \\
\hline$C y$ & 0.1 & 6.6 & -12.2 & 6.5 \\
& 0.2 & 3.2 & -9.8 & 7.6 \\
& 0.4 & -3.1 & -1.0 & 5.0 \\
\hline$C m$ & 0.1 & 3.9 & -8.8 & 5.8 \\
& 0.2 & -0.2 & -5.0 & 6.2 \\
& 0.4 & -1.5 & -3.1 & 5.5 \\
\hline$C x$ & 0.1 & 8.4 & -15.3 & 7.9 \\
& 0.2 & 4.6 & -11.7 & 8.1 \\
& 0.4 & -1.9 & -4.1 & 6.9 \\
\hline \hline
\end{tabular}

Table 4.10: Constants in the functional relationships for the maximum fluctuation in the force and moment coefficients (secondary response). Valid for $\rho_{2} / \rho_{1}<1.0 . M_{\infty}=0.63$.

\begin{tabular}{|c|c|r|r|r|}
\hline \hline Coefficient & Wake Width (w/c) & $\Phi_{1}$ & $\Phi_{2}$ & $\Phi_{3}$ \\
\hline$C y$ & 0.1 & -4.3 & 2.2 & 3.1 \\
& 0.2 & -6.4 & 5.7 & 1.7 \\
& 0.4 & 4.2 & -9.3 & 6.2 \\
\hline$C m$ & 0.1 & -2.5 & 0.7 & 2.8 \\
& 0.2 & -5.5 & 5.0 & 1.4 \\
& 0.4 & -1.2 & -0.8 & 3.0 \\
\hline$C x$ & 0.1 & -0.9 & -1.1 & 3.0 \\
& 0.2 & -3.9 & 3.3 & 1.7 \\
& 0.4 & 6.2 & -11.3 & 6.2 \\
\hline \hline
\end{tabular}

Table 4.11: Constants in the functional relationships for the maximum fluctuation in the force and moment coefficients (secondary response). Valid for $\rho_{2} / \rho_{1}<1.0 . M_{\infty}=0.87$. 
fluctuations scale with the maximum deflection of the shock wave. The magnitude of the fluid flux and the deflection of the shock wave are both found to scale with (1) the non-dimensional density wake width $w / c$ and (2) the density parameter $\rho^{*}$ however. The trends for the maximum fluctuation in the force and moment coefficients in viscous compressible flows are therefore identical to the trends observed in the inviscid incompressible flows.

- The magnitude of the force and moment fluctuations increase with flow Mach number. The magnitude of the azimuthal force coefficient fluctuations in particular can be scaled by the Prandtl-Glauert compressibility correction factor $\sqrt{1-M_{\infty}^{2}}$ for small wake widths $(w / c=0.1)$. This correction factor is inadequate to scale fluctuations caused by wake widths greater than $0.1 c$. The axial force and moment coefficients do not scale adequately with this correction factor either.

- The amplitude of the fluctuations in the force and moment coefficients after the density wake leaves the blade trailing edge are typically 2-10 times larger than the vortex shedding induced baseline fluctuations. These additional fluctuations are caused by a separation bubble on the blade suction surface formed by the density wake - boundary layer interaction. The amplitude of these fluctuations are found to scale with the maximum fluctuations in the blade separation point which in turn scales with (1) the normalized density wake width $w / c$ and (2) the density ratio $\rho_{2} / \rho_{1}$.

- The separation bubble is found to temporarily suppress vortex shedding for 2-3 convective time scale units after the density wake has left the blade trailing edge. The vortex shedding frequencies are consequently absent in the frequency response of the secondary fluctuations. 


\section{Chapter 5}

\section{Cascade Flow Model}

\subsection{Introduction}

CFD flow solvers are useful for investigating specific fluid flow phenomena however they are less useful for conducting parametric studies. This is due to the excessive computational run-times involved ${ }^{1}$. Furthermore investigation of changes in flow geometry is tedious as a new computational grid must be generated for each simulation. A simple flow model which incorporates the essential fluid dynamic features can however greatly reduce the time required to determine parametric trends and can also help assess the relative importance of flow geometry variables on a specified output variable.

A cascade flow model was therefore developed in this study in parallel with the CFD simulations to help determine the density wake induced blade force and moment fluctuations in an economical manner. The results from the viscous compressible CFD simulations in particular indicate a variety of fluid phenomena associated with the passage of density wakes; these cannot be easily modeled at the present time. As a first step we develop a simple model aimed at characterizing trends in the force and moment fluctuations for inviscid incompressible flows only. The assumptions and equations used in the model are presented below together with the results from a parametric analysis for the change in force and moment fluctuation magnitudes with varying density wake properties and cascade geometries.

\footnotetext{
${ }^{1}$ The investigation of time-accurate flow phenomena in particular requires extensive computational resources.
} 
The source code for the model is listed in Appendix D.

\subsection{Modeling Assumptions}

As stated earlier the model is focused on the density wake induced lift and moment in inviscid incompressible background flows. A combination of potential flow singularity solutions and a constant of proportionality determined from the inviscid CFD results is used to develop the model. In addition the model uses "quasi-steady aerodynamic" assumptions and is therefore at best valid for low reduced frequencies $(\mu<1.0$ [2]). The following assumptions are made to help further simplify the model:

1. The flow is two dimensional.

2. The cascade blades are unstalled so the flow always follows the blade surface.

3. Effects due to blade camber and thickness are neglected so that the cascade blades can be represented as flat plates.

4. The density wake can be represented as a row of counterrotating vortices which convect with the mean flow.

5. The influence of shed vorticity can be neglected in the transient response.

A schematic of the model is shown in Figure 5-1. The basic approach involves solving for the bound vorticity on the cascade flat plates such that the flow remains tangent to the plate surface. The flat plates are divided into $N$ discrete panels each with a bound vortex at the $1 / 4$ chord location. There are no changes in the bound vortex circulation strength on similar panels on different plates (i.e. zero interblade phase angle). The flow tangency condition is applied at the $3 / 4$ chord location of each panel. The cascade flat plates are inclined at stagger angle $\beta_{1}$ and have space-chord ratio $\sigma$. The free stream velocity is inclined at an angle $\alpha$ to the flat plates. 


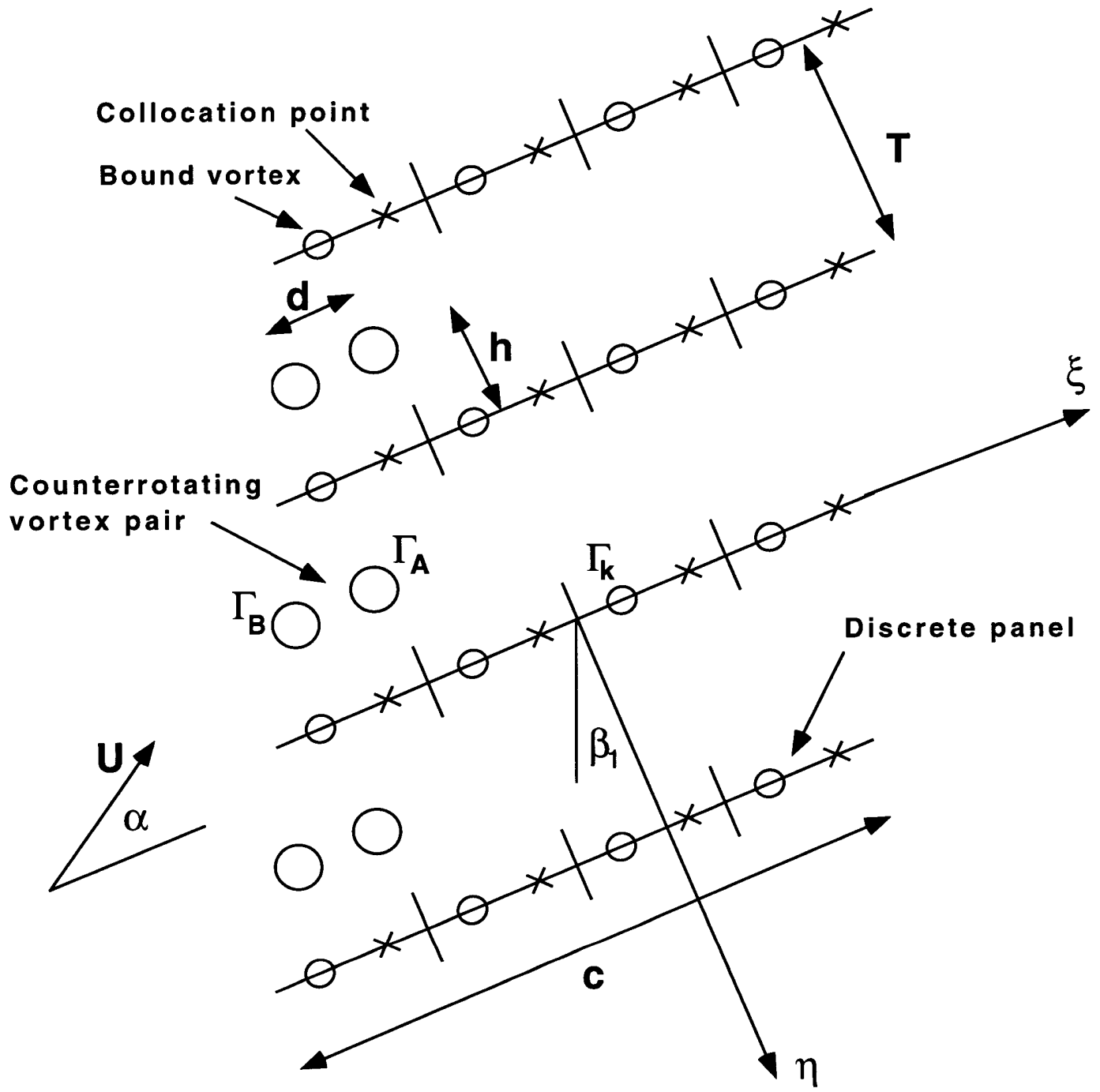

Figure 5-1: Model schematic indicating flat plate cascade and counterrotating vortex pairs. 
The convecting density wake is modeled by a row of counterrotating vortex pairs. This approach is motivated from the inviscid CFD flow visualization results which indicate the formation of counterrotating vortex pairs as the density wake interacts with the cascade pressure field (see Figure 1-4). The counterrotating vortices are staggered at angle $\beta_{1}$ (equal to the blade stagger angle) and are convected parallel to the flat plates at a fixed vertical offset $h$. The density wake width is represented by the spacing $d$ of the counterrotating vortices. The circulation $\Gamma_{A}$ and $\Gamma_{B}$ associated with each of the vortices in the counterrotating vortex pair is prescribed as a function of space and time.

\subsection{Induced velocities}

As stated earlier the basic approach used in the model involves solving for the circulation strength of each flat plate vortex panel such that the flow tangency condition is satisfied on the blade surface. The first step in this approach requires the evaluation of the induced velocity field of (1) the bound vortices and (2) the convecting counterrotating vortex pairs. For the case of an isolated flat plate the velocity $w$ (airfoil) induced by a single bound vortex of circulation strength $\Gamma$ at a location distance $r$ from the vortex is given by,

$$
w(\text { airfoil })=\frac{\Gamma}{2 \pi r}
$$

For the case of a cascade of flat plates with bound vorticity at $N$ discrete vortex panels the induced velocities from a row of vortices extending from $-\infty$ to $+\infty$ must be summed. The velocity $w$ (cascade) induced at any location $\zeta$ by a row of vortices strength $\Gamma_{k}$ located at the same relative position $\xi$ on a discrete vortex panel $k$, is given by

$$
w(\text { cascade })=\frac{-\imath \Gamma_{k}}{2 T} e^{\imath \beta_{1}} \operatorname{coth}\left(\pi \frac{\zeta-\xi}{T} e^{\imath \beta_{1}}\right)
$$


where $\beta_{1}$ is the plate stagger angle and $T$ is the plate spacing. This expression is derived in Kaufmann [10]. The real and imaginary parts of Equation 5.2 yield the induced velocity in directions parallel to and normal to the flat plate. For the counterrotating vortices which convect a distance $h$ above the flat plate, an equivalent expression can be obtained for the induced velocities. The velocity field $w$ (vortices) induced by the front row of vortices in the counterrotating pair (Figure 5-1) each with circulation strength $\Gamma_{A}$ and stagger angle $\beta_{1}$ is given by,

$$
w(\text { vortices })=\frac{-\imath \Gamma_{A}}{2 T} e^{\imath \beta_{1}} \operatorname{coth}\left\{\left(\pi \frac{\zeta-\xi}{T}+\imath \pi h\right) e^{\imath \beta_{1}}\right\}
$$

\subsubsection{Circulation Strength of The Counterrotating Vortices}

The circulation strength of the vortices in the counterrotating pair is assumed to be a function of the vorticity $\omega$ (of the vortex), an effective area $A_{\text {eff }}$, and time $\tau$, i.e.

$$
\Gamma=f\left(\omega, A_{\text {eff }}, \tau\right)
$$

The vorticity $\omega$ is assumed to change from zero to a fixed magnitude as the vortices convect through the cascade. This change in vorticity is governed by the interaction of the wake density gradient and the blade pressure gradient according to Equation 1.1. A hyperbolic tangent functional approximation is assumed to represent this change in vorticity. By interchanging the time dependency $\tau$ with the vortex position $\xi$ (by assuming a constant convection speed) the change in counterrotating vortex circulation strengths $\Gamma$ can now be expressed as, 


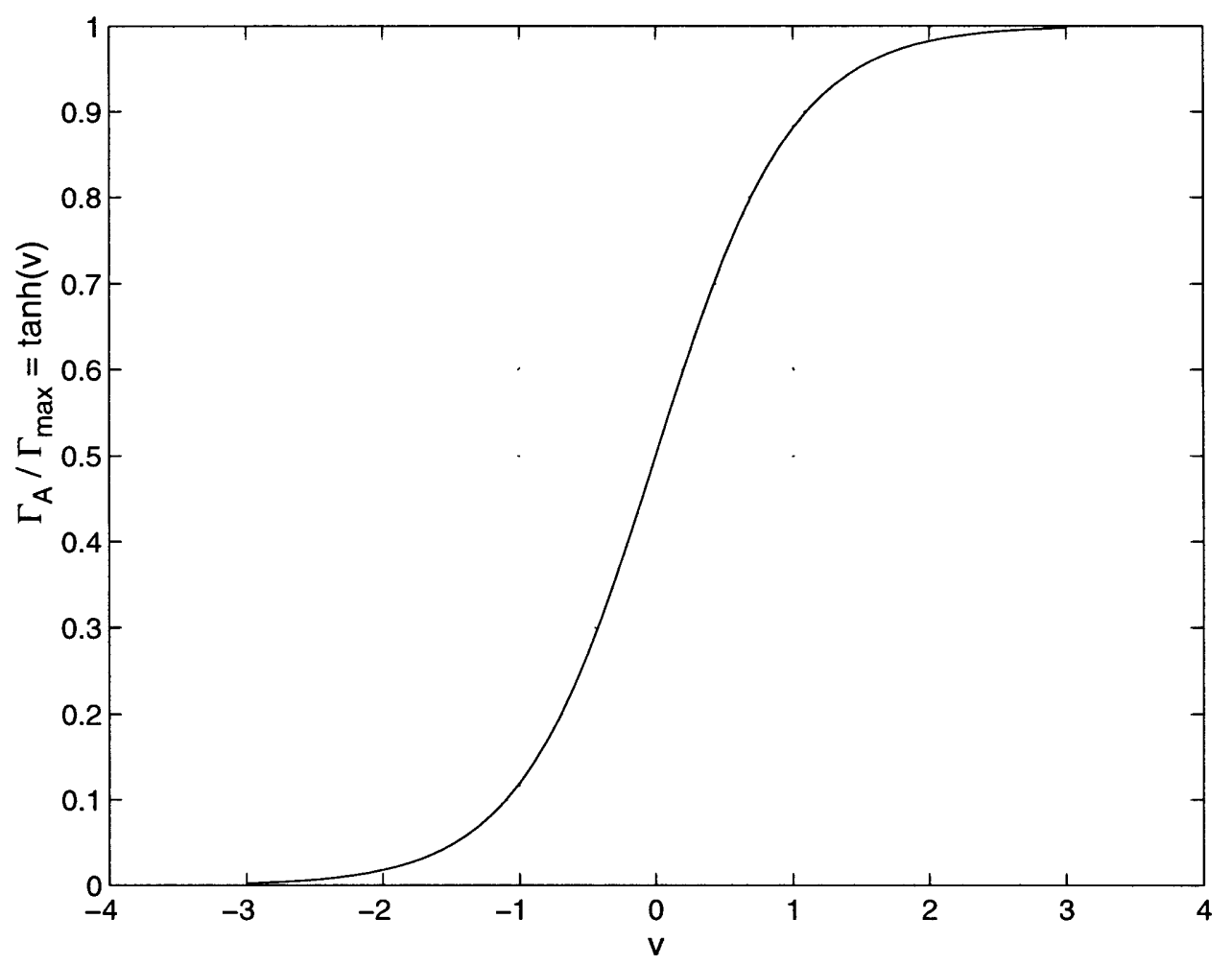

Figure 5-2: Change in circulation strength of the convecting vortices. $\mathrm{v}=s_{1} \xi+s_{2}$ where $\xi$ is the location of the convecting vortex and $s_{1}$ and $s_{2}$ are constants which ensure the tanh function is evaluated between -3 and +3 .

$$
\Gamma=0.5 \times \Gamma_{\max } \times\left(1+\tanh \left(s_{1} \xi+s_{2}\right)\right)
$$

where $\Gamma_{\max }$ is a constant dependent on the density wake properties and the cascade pressure distribution. The variables $s_{1}$ and $s_{2}$ are chosen such that the $\tanh$ function varies between -3 and +3 as the counterrotating vortex location $\xi$ varies between predetermined end points $l_{1}$ and $l_{2}$. These end points are obtained by considering the upstream and downstream decay of the cascade pressure field as a function of the blade row stagger angle, space-chord ratio and blade leading edge radius. For values of $\xi$ less than $l_{1}$ the circulation strengths are set to zero while for values greater than $l_{2}$ the circulation strengths are set to $\Gamma_{\max }$. The -3 to +3 range of the tanh function is chosen to ensure a smooth transition of $\Gamma_{A}$ and $\Gamma_{B}$ to the fixed end values (see Figure 5-2). 
$\Gamma_{\max }$ in Equation 5.5 is assumed to contain the influence of the blade row pressure gradient and the density gradient together with an effective area as follows,

$$
\Gamma_{\max }=f\left(\nabla p, \nabla \rho, A_{\text {eff }}\right)
$$

In the simplest case the functional relation for $\Gamma_{\max }, \nabla p, \nabla \rho$ and $A_{\text {eff }}$ is assumed to be linear, i.e.

$$
\Gamma_{\max }=K \frac{\left(C p_{s}-C p_{p}\right)}{T} \frac{\left(\rho_{2}-\rho_{1}\right)}{d / 2} \times\left(A_{e f f}\right)
$$

where $C p_{s}$ and $C p_{p}$ are the plate suction surface and pressure surface pressure coefficients ${ }^{2}$. $A_{e f f}$ is set equal to $T \times d / 2$ and $K$ is a constant of proportionality to be determined from the inviscid CFD results. Once $K$ is fixed the value of $\Gamma_{\max }$ is determined purely by the cascade geometry and density wake parameters.

\subsection{Solution Procedure}

The bound vortex circulation strengths $\Gamma_{k}$ at each discrete vortex panel $k$ and the induced velocities $F_{k}$ from the forcing terms (described shortly) at each panel $3 / 4$ chord location are related by an aero-influence coefficient matrix $A$ as follows,

$$
\{F\}=[A]\{\Gamma\}
$$

The $A$ matrix stores the vertical component of velocity induced at the $3 / 4$ chord location

\footnotetext{
${ }^{2}\left(C p_{s}-C p_{p}\right)$ is evaluated at the plate $1 / 4$ chord location.
} 
$\zeta(k)$ of each panel by the bound vortex of strength $\Gamma_{k}$ at each panel $1 / 4$ chord location $\xi(k)$. The $A$ matrix can be obtained by substituting appropriate values for $\zeta$ and $\xi$ in Equation 5.2 for a given cascade space-chord ratio and stagger angle.

The forcing vector $F_{k}$ contains (1) the vertical component of the free stream velocity $U_{n}$ and (2) the velocity induced by the counterrotating vortex pairs at the panel $3 / 4$ chord locations. The $F_{k}$ vector is constantly updated as the counterrotating vortex pairs convect through the cascade. The velocity induced by the counterrotating vortices at the plate leading edge is also added to $F_{k}^{3}$.

The unknown values of the panel bound vortex strengths $\Gamma_{i}$ can now be evaluated by inverting the aero-influence coefficient matrix $A$ as follows,

$$
\{\Gamma\}=[A]^{-1}\{F\}
$$

The cascade lift (force normal to the flat plate along the $-\eta$ direction) ${ }^{4}$ and the moment (clockwise positive about the plate mid-chord) is then obtained using,

$$
\begin{aligned}
L & =\rho U \sum_{k=1}^{N} \Gamma_{k} \\
M & =\rho U \sum_{k=1}^{N} \Gamma_{k} \times \xi(k)
\end{aligned}
$$

Note the influence of shed vorticity and the unsteady contribution to lift and moment from the unsteady circulation $d \Gamma_{k} / d t$ is not included in this model. The remaining sections describe the validation of the model and the parametric studies conducted with different density wakes and cascade geometries.

\footnotetext{
${ }^{3}$ This represents the influence of the counterrotating vortices on the free stream flow.

${ }^{4}$ The term "lift" will be used subsequently instead of "force" to help indicate this direction.
} 


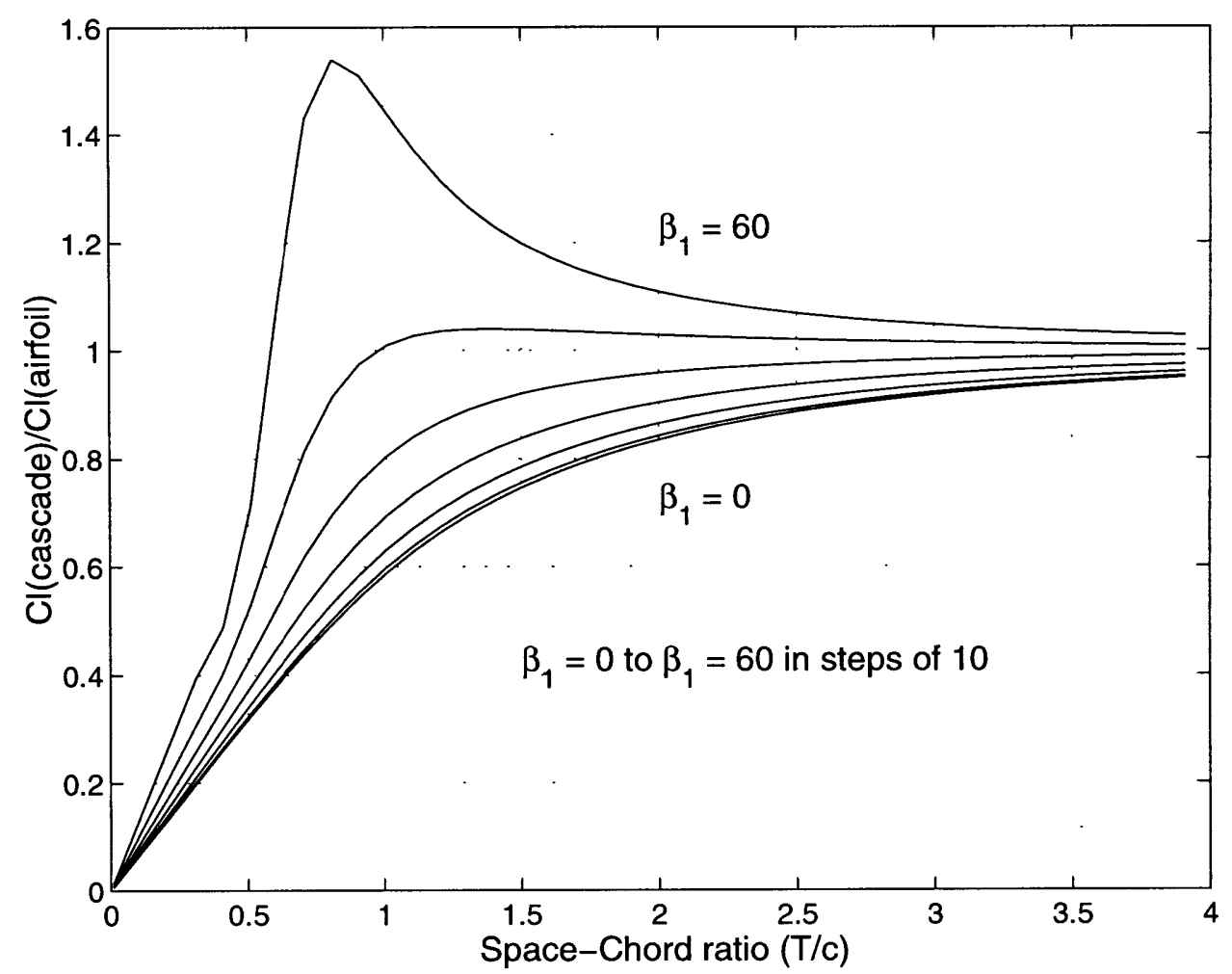

Figure 5-3: Cascade model results for the change in cascade interference coefficient $C l_{\text {cascade }} / C l_{\text {arforl }}$ with space-chord ratio and stagger angle. No. of panels $=1$.

\subsection{Steady State Model Validation}

The steady state lift coefficient predicted by the model (i.e. with no convecting density wakes) is shown in Figure 5-3 for a range of cascade space-chord ratio's and stagger angles. The cascade lift coefficient $C l_{\text {cascade }}$ shown here is normalized by the lift coefficient of an isolated flat plate airfoil $C l_{\text {airfoil }}$. A single vortex panel is used in this case. All curves show an asymptotic value of 1.0 as the space-chord ratio is increased. This is as expected and confirms the cascade lift coefficient approaches the flat plate lift coefficient in the limit of large space-chord ratios. Also notice the large increase in cascade lift coefficient for stagger angles $\beta_{1}>45$ degrees and space-chord ratio $\sigma \approx 1.0$. This is due to the increased flow restriction between the adjacent flat plates at these high values.

The corresponding plot obtained using a conformal mapping method by Weinig [7] is shown in Figure 5-4. The overall trends compare well however the numerical values differ significantly at the higher stagger angles. Better agreement can be obtained using 5 vortex 


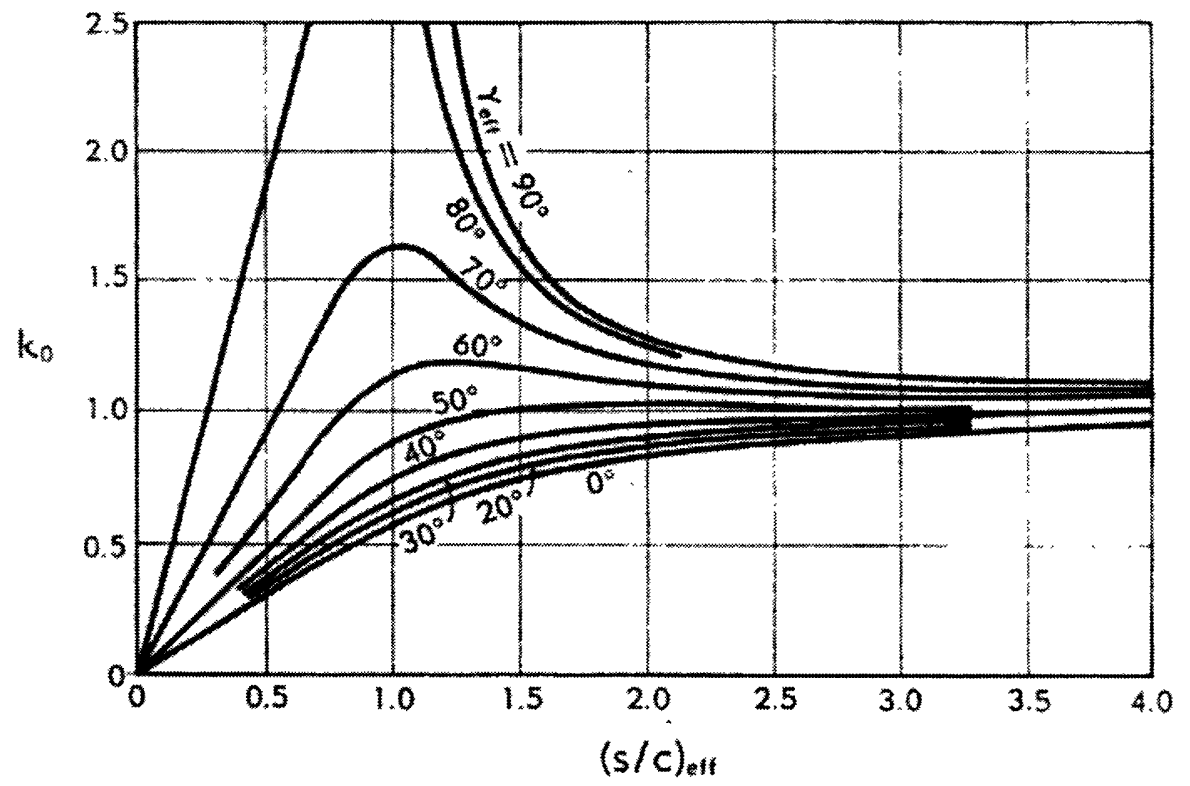

Figure 5-4: Weinig's conformal mapping prediction for the cascade interference coefficient $k_{o}$ $\left(C l_{\text {cascade }} / C l_{\text {arforl }}\right)$ as a function of stagger angle $\gamma_{e f f}$ and space-chord ratio $(s / c)_{e f f}$.

panels to represent the flat plates. The corresponding results shown in Figure 5-5 now compare to within $2 \%$ of Weinig's results ${ }^{5}$. The model results for the steady state moment coefficient about the plate mid-chord location is plotted in Figure 5-6 normalized about the moment coefficient for an isolated airfoil. An equivalent theoretical prediction could not be found for comparison purposes for this case. The moment coefficient nevertheless approaches the thin airfoil predicted value in the limit of large space-chord ratio.

The results presented here confirm the model can adequately predict the numerical values and trends for the cascade steady state lift and moment coefficients. The model results for the quasi-steady lift and moment profiles induced by density wakes are presented next.

\footnotetext{
${ }^{5}$ Results for stagger angles greater than 60 degrees could not be obtained using this model since the aero-influence coefficient matrix $A$ becomes ill-conditioned and cannot be inverted.
} 


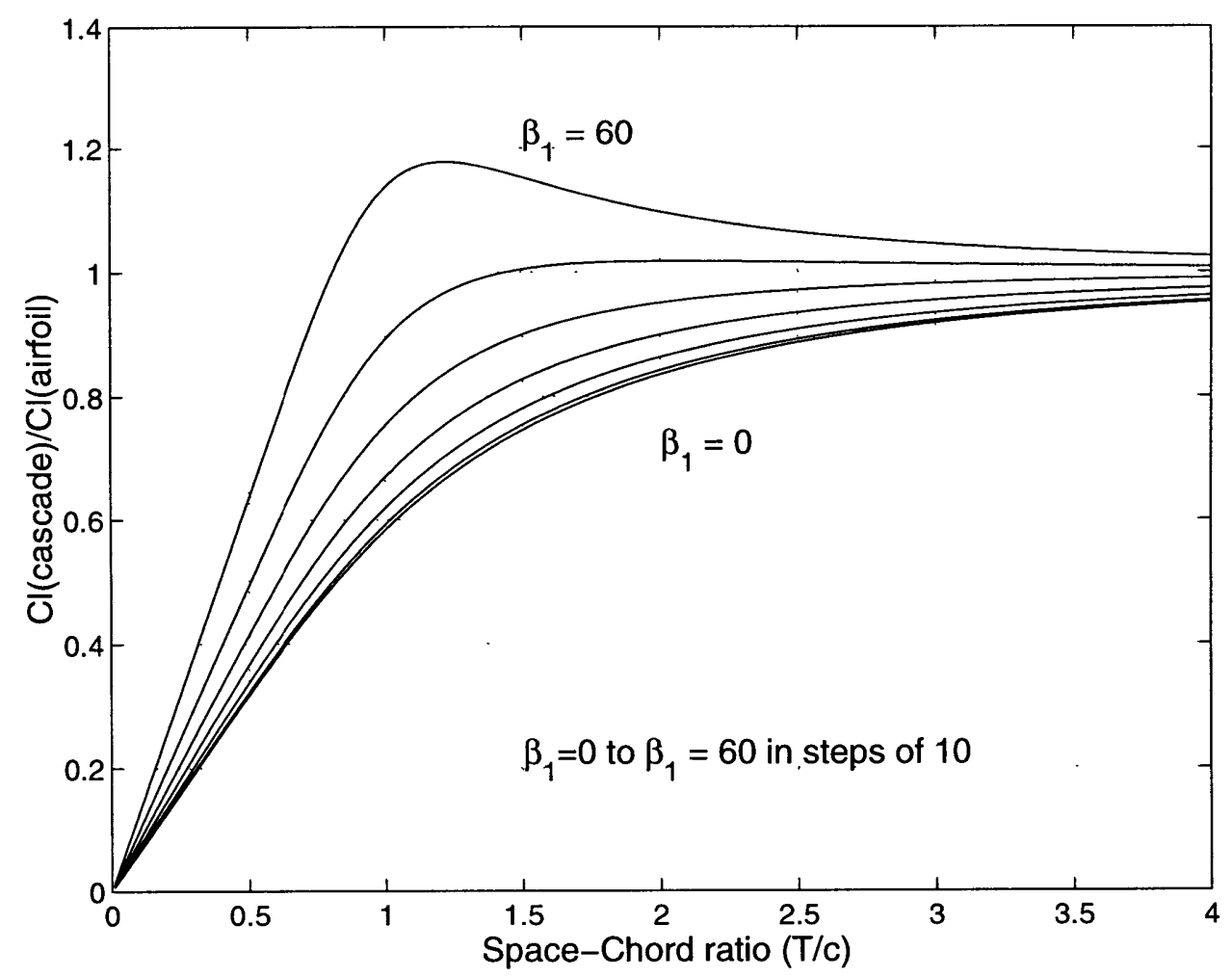

Figure 5-5: Cascade model results for the change in cascade interference coefficient $C l_{\text {cascade }} / C l_{\text {arffoll }}$ with space-chord ratio and stagger angle. No. of panels $=5$.

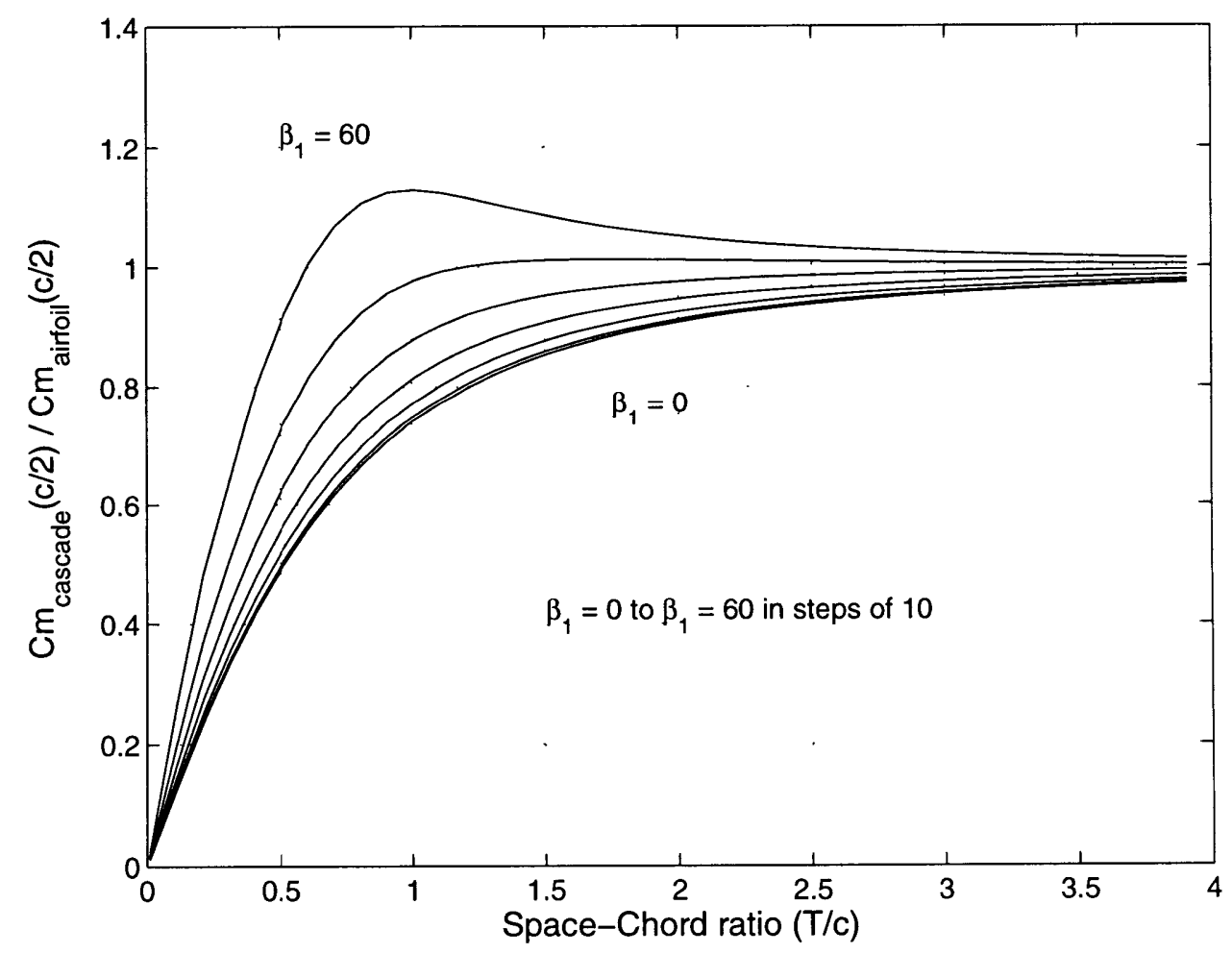

Figure 5-6: Cascade model results for the change in cascade interference coefficient $C m_{\text {cascade }} / C m_{\text {arffol }}$ with space-chord ratio and stagger angle. No. of panels $=5$. 


\begin{tabular}{|l|l|}
\hline \hline Property & Value \\
\hline Space/chord ratio & 0.58 \\
Stagger angle & $15.0 \mathrm{deg}$. \\
Max. blade thickness & $0.037 c$ \\
Blade leading edge radius & $0.0075 c$ \\
\hline \hline
\end{tabular}

Table 5.1: Properties of the NACA4F cascade geometry used for the inviscid CFD tests.

\begin{tabular}{|l|l|}
\hline \hline Variable & Value \\
\hline Number of vortex panels $(N)$ & 20 \\
Space/chord $(s / c)$ & 0.58 \\
Cascade stagger angle $\left(\beta_{1}\right)$ & 15.0 \\
Counterrotating vortex spacing $(d)$ & $0.1 c$ \\
Density ratio $\left(\rho_{2} / \rho_{1}\right)$ & 0.5 \\
Upstream infl. of press. field $\left(l_{1}\right)$ & $0.3 c$ upstream of l.e. \\
Downstream infl. of press. field $\left(l_{2}\right)$ & $0.4 c$ downstream of l.e. \\
Incidence angle $(\alpha)$ & 5 deg. relative to blade \\
Circulation strength constant $(K)$ & 0.32 \\
\hline \hline
\end{tabular}

Table 5.2: Cascade model parameters used to determine the flat plate force and moment coefficient fluctuation during passage of a density wake of width $0.1 c$ and density ratio 0.5 .

\subsection{Quasi-Steady Model Validation}

The quasi-steady model validation involves comparison of the model prediction for the density wake induced flat plate lift and moment fluctuation with a corresponding inviscid CFD prediction. The model parameters are set to match the cascade geometry properties and density wake properties of a typical inviscid CFD calculation. For the cascade properties, a NACA4F blade geometry was chosen with properties listed in Table 5.1. For the density wake a width of $0.1 c$ and density ratio 0.5 was considered a representative case. The model parameters corresponding to this set of cascade and density wake properties is listed in Table 5.2.

The model results and the inviscid CFD results for the force and moment fluctuations are compared in Figure 5-7. The dashed line represents the model prediction and the solid line represents the inviscid CFD prediction. The change in the lift and moment coefficients are shown as percentage changes from the steady state values. Note the definition for the 
model moment coefficient (positive clockwise about the mid-chord) was changed to positive counter-clockwise about the mid-chord for comparison purposes with the inviscid CFD results $^{6}$. The maximum fluctuation in the model lift prediction is matched to the inviscid results by adjusting the value of the circulation constant $K$ (Equation 5.7). 20 vortex panels are used to discretize the cascade flat plates for this case. Use of more than 20 panels is found to change the amplitude and frequency response by negligible amounts.

Significant deviation can be seen between the model and the inviscid CFD predictions. The maximum change in the lift response occurs at a time lag $\Delta \tau \approx 0.4$ after the corresponding maximum change in the inviscid CFD lift results. Similarly the maximum change in the moment occurs $\Delta \tau \approx 0.2$ before the corresponding maximum change in the inviscid CFD moment results. The initial decrease/increase in lift/moment compares well however the return to the steady state values does not compare. In addition the lift peak at $\tau=0.1$ predicted by the model has a larger amplitude and occurs later than the similar peak predicted in the inviscid CFD results. The similar peak in the moment response is not captured by the model. Furthermore the overall maximum change in the moment is under predicted. The following list of model features have been highlighted as possible reasons for the observed discrepancies.

- The maximum change in the lift and moment coefficients occur as the density wake passes over the blade leading edge region. The blade leading edge geometry and corresponding pressure distribution pattern is therefore critical to the overall response profile. The model however replaces the finite blade radius with an infinite radius and consequently an infinite pressure peak. It may not be possible therefore to adequately capture the initial response shape with a flat plate geometry.

- The initial rise in the lift response curves was found to be governed by the induced velocity field of the clockwise vortex (vortex B) of the counterrotating vortex pair. The clockwise vortex induces an upwash velocity at the plate leading edge when it is located downstream of the plate leading edge. This results in an increase in the

\footnotetext{
${ }^{6}$ Also note the lift coefficient is defined normal to the flat plate whereas the force coefficient obtained from inviscid CFD simulations is defined along the azimuthal coordinate direction. The difference is absorbed into the model constant $K$.
} 

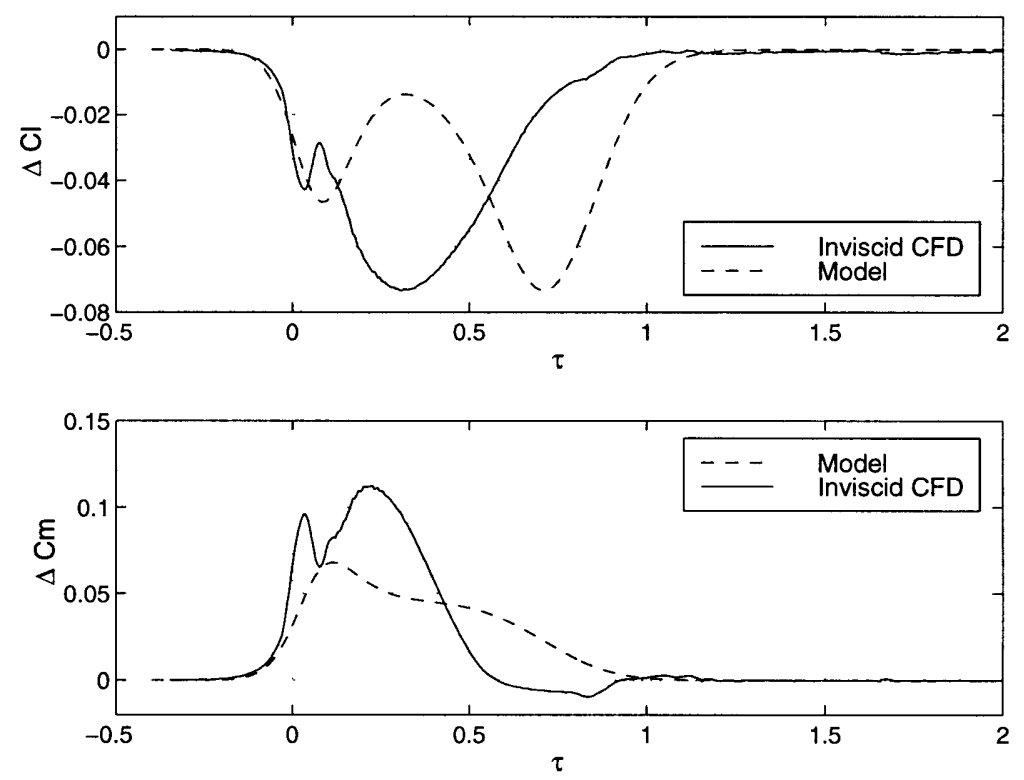

Figure 5-7: Comparison of the quasi-steady model results and the inviscid CFD results for the fluctuation in the blade force and moment coefficients during passage of a density wake of width $0.1 c$ and density ratio 0.5 . $\Delta C l=\left(C l_{\text {max }}-C l_{\text {mean }}\right) /\left(C l_{\text {mean }}\right)$, $\Delta C m=\left(C m_{\max }-C m_{\text {mean }}\right) /\left(C m_{\text {mean }}\right)$.

flow angle of attack and hence an increase in the lift coefficient. The model moment response does not capture this effect however. Since the moment is calculated about the plate mid-chord there must be a relative difference in induced velocity at the collocation points upstream and downstream of the mid-chord for a visible fluctuation in the moment to occur. The induced velocity of the clockwise vortex at the leading edge is however applied equally to all $3 / 4$ chord collocation locations on the vortex panels. There is little if no net difference in the moment therefore due to the increase in flow angle of attack. The induced velocity at the plate leading edge should therefore be weighted at the vortex panel collocation locations for improved comparison with the inviscid CFD results. This may also help to reduce the magnitude of the initial increase in the lift coefficient.

- The lag between the model and inviscid CFD results may be due to the influence of shed vorticity which is not accounted for in the model.

The sensitivity of the lift and moment response curves to changes in the density wake properties and the cascade geometry is examined next. 


\subsection{Parametric Results}

\subsubsection{Lift and Moment Sensitivity to Density Wake Properties}

The NACA4F cascade geometry is used as the baseline geometry for the sensitivity analysis. The circulation constant $K$ is fixed at 0.322 . This sets the maximum fluctuation in lift coefficient to $7.3 \%$ and the maximum fluctuation in moment coefficient to $-6.8 \%$ for a density wake of width $0.1 c$ and density ratio 0.5 (see Figure $5-7$ ).

The results for the maximum fluctuation in the lift and moment coefficients with varying density wake width and density ratio are plotted in Figure 5-8. The variation in density ratio is represented here by Marbles' density parameter $\rho^{*}$ (Equation 1.2). The model data is indicated by markers joined by straight lines. The corresponding inviscid CFD results are shown superimposed as dashed lines. The model trends agree well with the inviscid CFD results. In particular the model values for the maximum fluctuation in azimuthal force coefficient compare to within $2 \%$ with the CFD numerical values for density parameter $\rho^{*}<0$.

It is interesting to note how the model predicts the non-linearity in the maximum lift and moment fluctuations for large values of density parameter and wake width similar to the non-linearity observed in the inviscid CFD results. Initial investigations indicate the non-linearity to originate from the expressions for the counterrotating vortex circulation strengths ${ }^{7}$ (Equation 5.5).

\subsubsection{Lift and Moment Sensitivity to Cascade Geometry}

The density wake width and density ratio were held constant at $0.1 c$ and 0.5 respectively as the cascade stagger and space-chord ratio were varied. The circulation constant $K$ was again fixed at 0.322 .

The results for the maximum fluctuation in the lift and moment coefficients with vary-

\footnotetext{
${ }^{7}$ A systematic analysis of the model parameters can help to isolate the precise source of this non-linearity.
} 

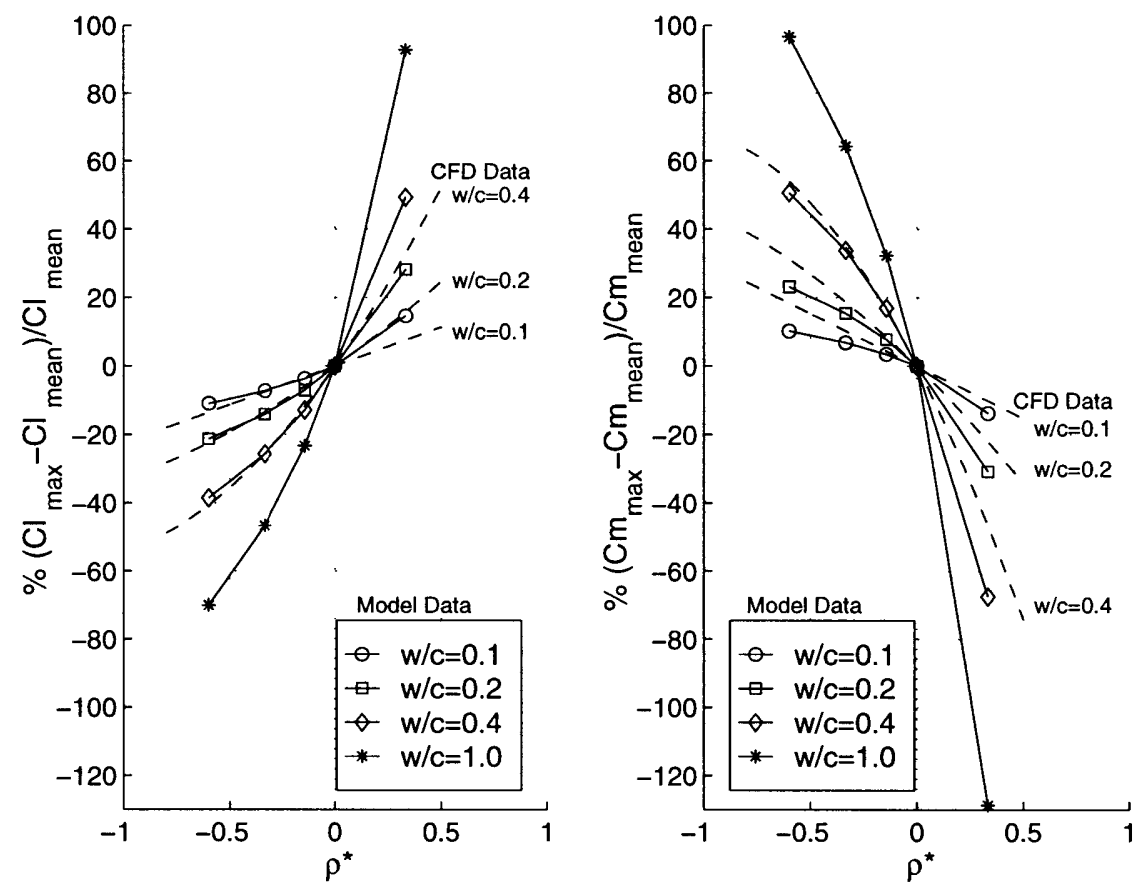

Figure 5-8: Cascade lift and moment fluctuation sensitivity to density wake width $w / c$ and density parameter $\rho^{*}$ (measure of density ratio). The NACA4F cascade geometry is used for all tests. Solid lines indicate the model results. Dashed lines indicate the inviscid CFD results.

ing cascade space-chord ratio and stagger angle are plotted in Figure 5-9. The lift coefficient is more sensitive to the cascade space-chord ratio than to the stagger angle. This may be because the counterrotating vortices convect at the same stagger angle as the cascade ${ }^{8}$. The reduction in lift coefficient fluctuation as the space-chord ratio is increased is most likely due to the increased distance between the counterrotating vortices and the blade surfaces. The moment coefficient fluctuation also decreases with increasing space-chord ratio and shows greater sensitivity to the cascade stagger angle.

\subsection{Summary}

A model to predict the density wake induced force and moment fluctuations on cascade blade rows has been described in this Chapter. The model is developed using the following main assumptions:

\footnotetext{
${ }^{8}$ This is an assumption in the model.
} 

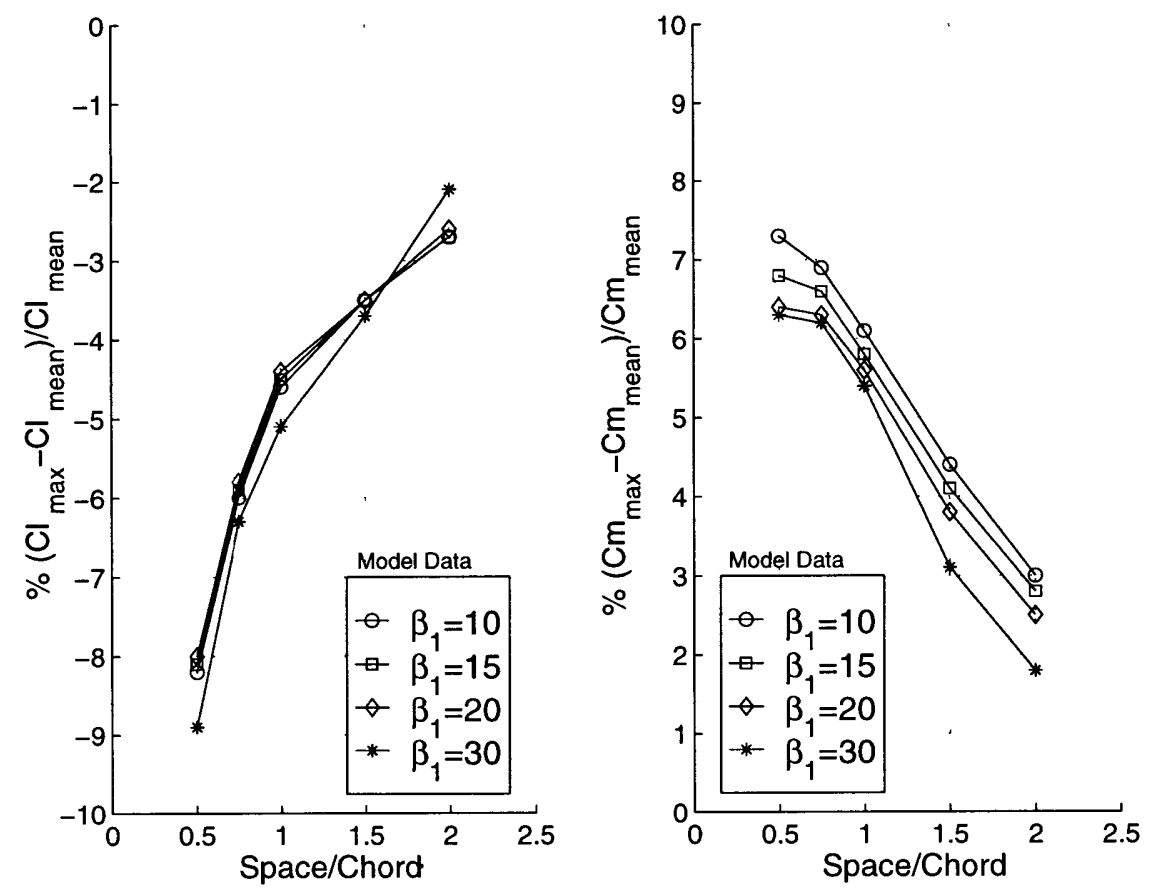

Figure 5-9: Cascade lift and moment sensitivity to cascade stagger angle and space-chord ratio. Density wake of width $0.1 c$ and density ratio 0.5 is used for all tests.

1. Effects due to blade camber and thickness can be neglected so that the cascade blades can be represented as flat plates.

2. The density wake can be represented as a row of counterrotating vortices which convect with the mean flow.

3. The influence of shed vorticity can be neglected.

The model predicts the maximum fluctuation in the lift and moment coefficients to (1) increase with density wake width, (2) increase with density parameter $\left|\rho^{*}\right|,(3)$ decrease with increasing space--chord ratio and (4) remain relatively insensitive to blade stagger angle.

The model predicted trends for the force and moment fluctuations with varying density wake properties and fixed cascade geometry follow the same trends as the inviscid CFD results. Moreover for the case of density parameter $\rho^{*}<0$ the numerical values for the maximum fluctuation in the lift coefficient agree to within $2 \%$ of the inviscid CFD results. The model results for the moment coefficient however under predict the inviscid CFD results by up to $50 \%$. The fluctuations in the moment coefficient are sensitive to relative changes 
in the flow about the mid chord position (by definition). The model does not adequately capture the extra length parameter (moment arm) required to determine the moment coefficient. The assumptions in the model regarding the magnitude of the induced velocity at the vortex panel collocation points needs to be re-examined to determine the origin of this difference. 


\section{Chapter 6}

\section{Conclusions And FurTher Work}

\subsection{Summary}

The density wake induced force and moment fluctuations in a compressor blade row have been characterized for viscous compressible flows ranging from $M_{\infty}=0.15$ to $M_{\infty}=0.87$ and Reynolds numbers $\approx 700,000$. Cause and effect relationships have been established between the shape of the force and moment response profiles and characteristic observable flow field features. Parametric trends have also been established for the amplitude and frequency of the force and moment fluctuations with varying density wake properties and free stream Mach number. These trends have been quantified using simple functional relationships.

In parallel with the CFD simulations a simple cascade flow model has also been developed to provide an economical method to investigate the trends in the blade force and moment fluctuations with changes in density wake properties and cascade geometries. The cascade model has been developed from a combination of potential flow singularity solutions and a constant of proportionality based on the inviscid CFD results.

The conclusions reached in this research are summarized in the next section. This is followed by suggestions for further work. 


\subsection{Conclusions Based On The Viscous Results}

- For the viscous flows at $M_{\infty}=0.15,0.53$ and 0.63 the mechanism for the force and moment fluctuations consist of the fluid flux directed to the blade surface (this is the same as for inviscid incompressible flows). For flows with shock waves however $\left(M_{\infty}=0.87\right)$ the force and moment fluctuations scale with the maximum deflection of the shock wave. The magnitude of the fluid flux and the magnitude of the shock wave deflection are both found to scale with (1) the non-dimensional wake width $w / c$ and (2) the density parameter $\rho^{*}$. The trends for the maximum fluctuation in the force and moment coefficients in viscous compressible flows are therefore identical to the trends observed in the inviscid incompressible flows.

- The magnitude of the force and moment fluctuations are found to increase with flow Mach number. The magnitude of the azimuthal force coefficient fluctuations in particular scale with the Prandtl-Glauert compressibility factor $\sqrt{1-M_{\infty}^{2}}$ for small wake widths $(w / c=0.1)$. The axial force coefficient and the moment coefficient do not adequately scaled with the Prandtl-Glauert factor however. Additional compressibility scaling factors must be investigated for these coefficients and for fluctuations induced by larger density wake widths.

- The viscous compressible flow environments allow for additional sources of blade force and moment fluctuations that do not occur in inviscid incompressible flows. In particular (1) periodic vortex shedding at the blade trailing edge and (2) separation bubbles on the blade suction surface are found to generate significant force and moment fluctuations. These flow features represent possible additional sources for high cycle fatigue failure and require further investigation.

\subsection{Conclusion Based On The Cascade Model Results}

- The cascade flow model developed in this research confirms the feasibility of a simple model to capture the essential features of the density wake induced force and moment

fluctuations. The model can aid the designer to conduct parametric studies and predict bounds for the maximum force and moment fluctuations for a range of density 
wake properties and cascade geometries.

\subsection{Suggestions For Further Work}

The suggestions for further work are listed below.

- The current research was focused on low density wakes convecting parallel to the axial flow direction interacting with a single cascade blade row in a 2-dimensional flow environment. It is possible to expand this scenario to include:

1. Multiple density wakes.

2. Density wakes convecting at varying angles to the axial flow direction.

3. Multiple blade rows.

4. 3-dimensional flow.

A careful study should precede further work with additional independent variables however in order to ascertain a practical yet relevant parameter space of computational simulations.

- The effect of changes in Reynolds number on the density wake induced force and moment fluctuations has not been investigated in this research. In particular the impact of density wakes in low Reynolds number flows typical for fan blades should be analyzed as high cycle fatigue failure is most common here.

- The viscous compressible flow simulations conducted in this research have not modeled the aeroelastic feedback due to blade vibrations. A fluid-structure coupled flow solver should be used to assess the impact of blade vibrations on the density wake induced force and moment fluctuations.

- The density wake induced force and moments determined in this study must be validated with experimental results. Major obstacles here may consist of the lack of a practical methodology to (1) generate density wakes with specific width and density ratio and (2) to instrument blades to accurately determine static pressure fluctuations. An effective solution to these 2 problems may aid the development of a simple 
experimental facility to investigate the density wake induced forces and moments fluctuations. 


\section{BIBLIOGRAPHY}

[1] Anderson, J. D. JR. Fundamentals Of Aerodynamics. McGraw-Hill, Inc. 1991. pp. $542-545$.

[2] Cesnik, C. E. S. Personal communication.

[3] Chieng, C. C. And Launder, B. E. "On the Calculation of Turbulent Heat Transport Downstream from an Abrupt Pipe Expansion". Numerical Heat Transfer, Vol. 3, 1980, pp.189-207.

[4] Covert, E. E. Personal Communication.

[5] Giles, M. B. "Non-Reflecting Boundary Conditions for the Euler Equations". CFDLTR-88-1, Computational Fluid Dynamics Laboratory, Massachusetts Institute of Technology, February 1988.

[6] Gostelow, J. P. Cascade Aerodynamics. Pergamon Press Ltd. 1984. pp. 171-172.

[7] Hawthorne, W. R. (ED.) Aerodynamics Of Turbines And Compressors. Princeton University Press. 1964. pp. $32-34$.

[8] Heinemann, H. J. and Butefisch, K. A. "Determination of the Vortex Shedding Frequency of Cascades with Different Trailing Edge Thickness". AGARD CP-227, Paper 35 (1978).

[9] Hoying, D. A. "Blade passage Flow Structure Effects On Axial Compressor Rotating Stall Inception ". PhD. Thesis, Massachusetts Institute of Technology, Department of Aeronautics and Astronautics, September 1996. 
[10] Kaufmann, W. Fluid Mechanics. McGraw-Hill Book Company, Inc., 1963, pp.354356.

[11] Kemp, N. H. And Sears, W. R. "The Unsteady Forces Due to Viscous Wakes in Turbomachines". Journal of Aeronautical Sciences, Vol.22, No.7, July 1955, pp.478483.

[12] Kerrebrock, J. L. ANd MikolajczaK, A. A. "Intra-Stator Transport of Rotor Wakes and its Effect on Compressor Performance". ASME Paper 70-GT-39, 1970.

[13] Launder, B. E. And Spalding, D. B. Computer Methods in Applied Mechanics and Engineering. Vol. 3. 1974. pp. 269-289.

[14] Lawaczeck, O. and Heinemann, H. J. "Von Karman Vortex Streets in the Wakes of Subsonic and Transonic Cascades". AGARD CP-177, Paper 28 (1976)

[15] Manwaring, S. R. And Wisler, D. C. "Unsteady Aerodynamics and Gust Response in Compressors and Turbines". ASME Paper 92-GT-422, 1992.

[16] Marble, F. E. "Response of a Thin Airfoil Encountering a Strong Density Discontinuity". Journal of Fluids Engineering, Vol. 115, December 1993, pp.580-589.

[17] Peraire J. Personal communication.

[18] Platzer, M. F. "Unsteady Flows In Turbomachines - A Review of Current Developments". AGARD CP-227, Paper 33 (1978).

[19] Ramer, B. E. "Aerodynamic Response of Turbomachinery Blade Rows to Convecting Density Distortions". S.M. Thesis, Massachusetts Institute of Technology, Department of Aeronautics and Astronautics, December 1996.

[20] Steger, J. L. And Sorenson, R. L. "Automatic Mesh-Point Clustering Near a Boundary in Grid Generation with Elliptic Partial Differential Equations". Journal of Computational Physics, Vol. 33, 1979, pp.405-410.

[21] Ramer, B. E., Wijesinghe, H. S., Tan, C. S.,Covert, E. E. "Aerodynamic Response of Turbomachinery Blade Rows to Convecting Density Wakes". Proceedings of the ASME Aerospace Division, Presented at the International Mechanical Engineering Congress and Exposition, Dallas, Texas, AD-VOL.55, November 1977. 
[22] TAM, C. K. W. AND WebB J. C. "Dispersion-Relation-Preserving Finite Difference Schemes for Computational Acoustics". Journal of Computational Physics, Vol. 107, 1993, pp.262-281.

[23] Valkov, Theodore V. "Control of Unsteady Flow in a Stator Blade Row Interacting with Upstream Moving Wakes". S.M. Thesis, Massachusetts Institute of Technology, Department of Aeronautics and Astronautics. Also GTL Report No. 255, May 1992.

[24] Wijesinghe, H. S. "Aerodynamic Response of Turbomachinery Blade Rows to Convecting Density Wakes". Final report for AFOSR Contract No. F49620-94-1-0202, MIT Gas Turbine Lab, December 1997.

[25] Williamson, C.H.K. And Roshko, A. "Vortex Formation In The Wake Of An Oscillating Cylinder". Journal of Fluids and Structures, Vol.2, July 1988, pp.355-381.

[26] Wisler, D. C. "Core Compressor Exit Stage Study, Volume I - Design Report". NASA CR-135391, NASA Lewis Research Center, December 1977.

[27] Basic Research Issues in Aerodynamics, Structural Dynamics and Control of High Cycle Fatigue. Summary of a Workshop held at the Gas Turbine Laboratory, MIT, October 1995. 


\section{ApPENDix A}

\section{BASELINE FlOW ReSUlts}

The following plots for baseline flows $M_{\infty}=0.15,0.53,0.63$ and 0.87 are included in this Appendix.

- Time averaged blade pressure distributions.

- Azimuthal force coefficient $C y$, axial force coefficient $C x$ and moment coefficient $C m$ fluctuations.

- Time averaged boundary layer properties: non-dimensional momentum thickness $\theta / c$, non-dimensional displacement thickness $\delta^{*} / c$ and skin friction coefficient $C f$.

Please note the following.

- The moment coefficient is defined positive clockwise and is calculated about the blade coordinates $x / c=0.42$ and $y / c=0.29$ (see Figure 2-4).

- The boundary layer properties are plotted vs. the surface distance to help increase resolution at the blade leading and trailing edge regions. 


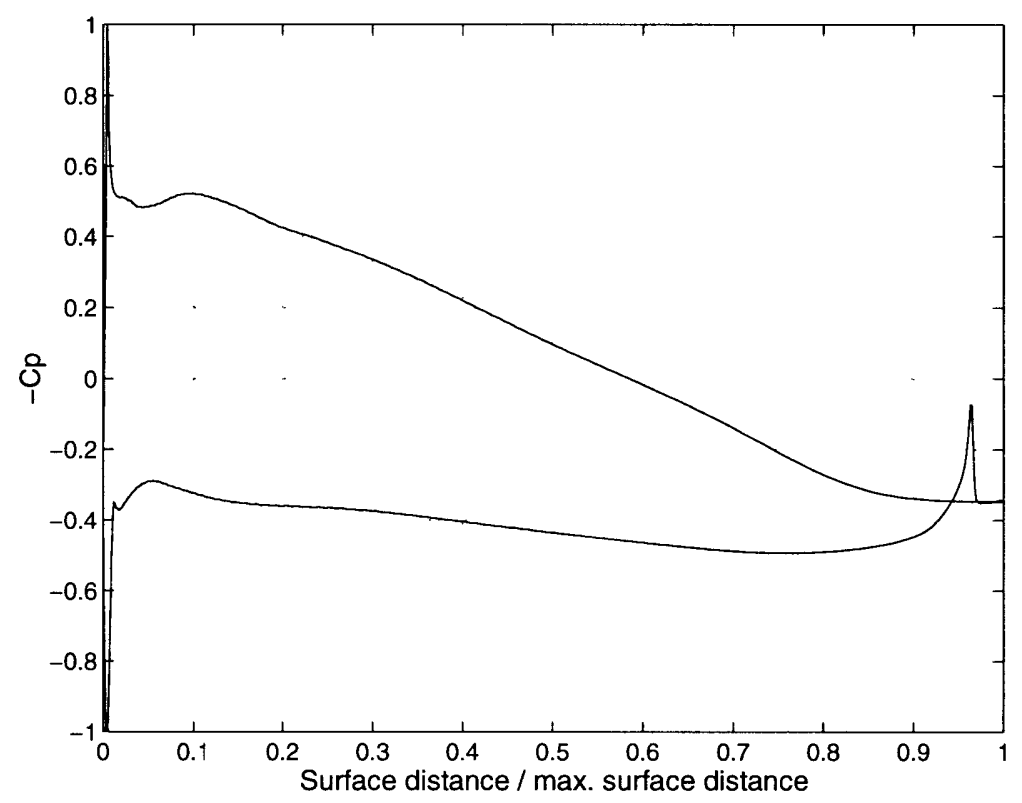

Figure A-1: Time averaged blade pressure distribution. $M_{\infty}=0.15$.

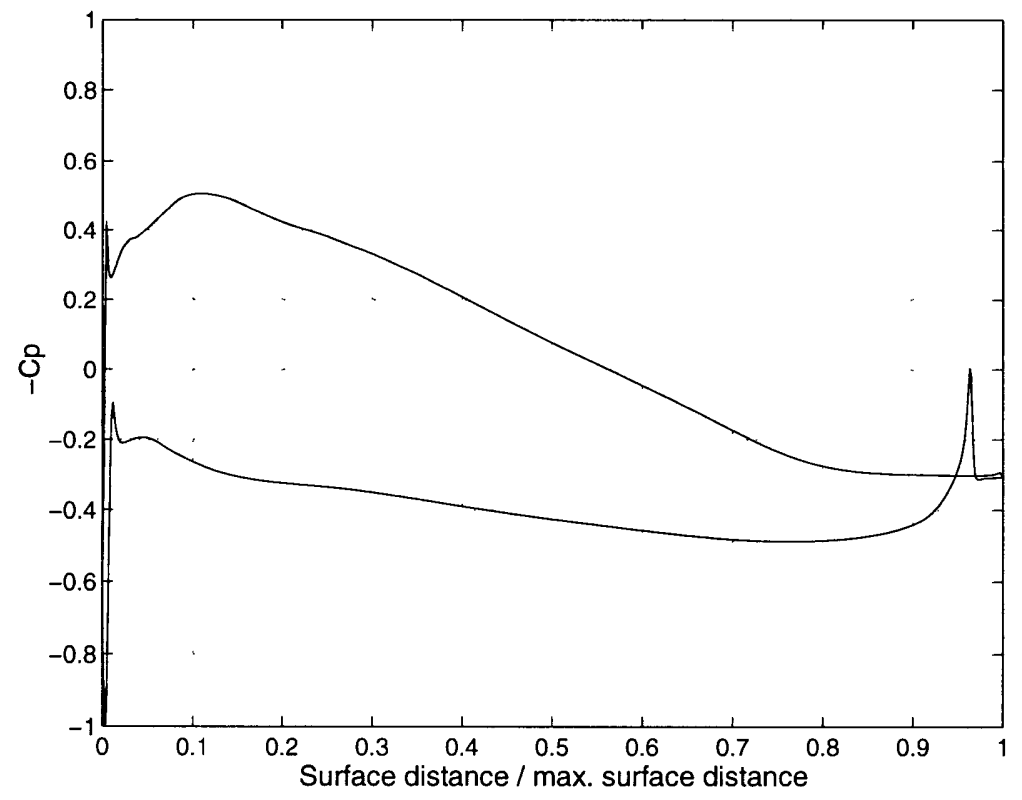

Figure A-2: Time averaged blade pressure distribution. $M_{\infty}=0.53$. 


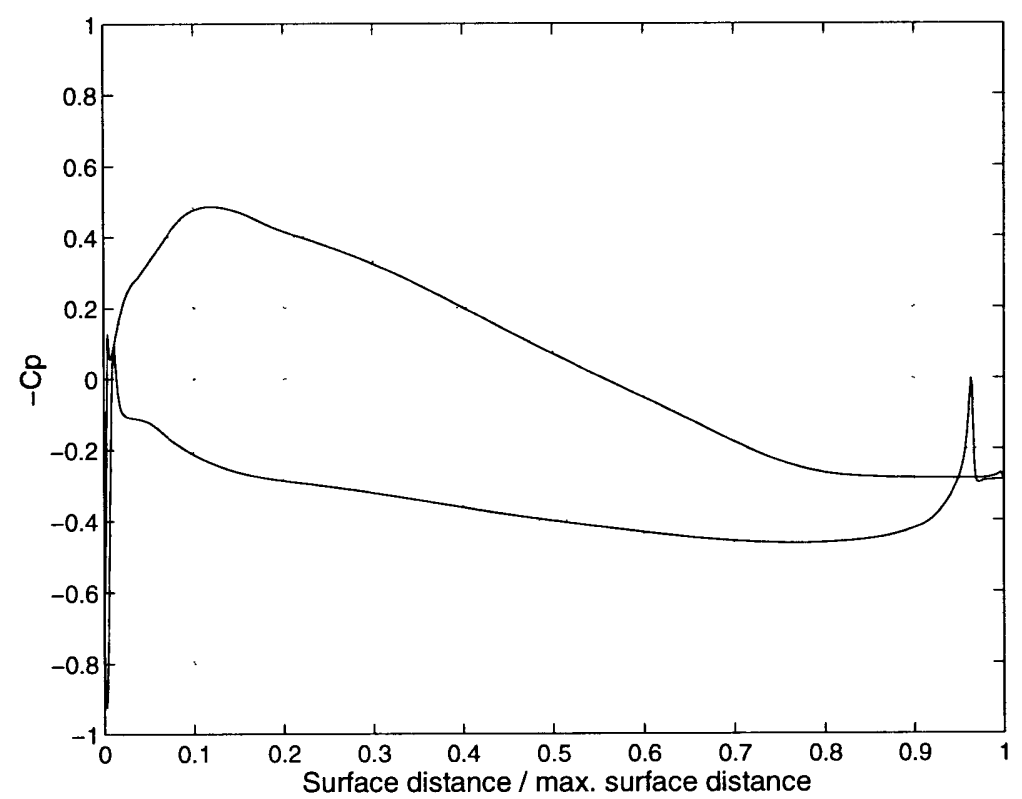

Figure A-3: Time averaged blade pressure distribution. $M_{\infty}=0.63$.

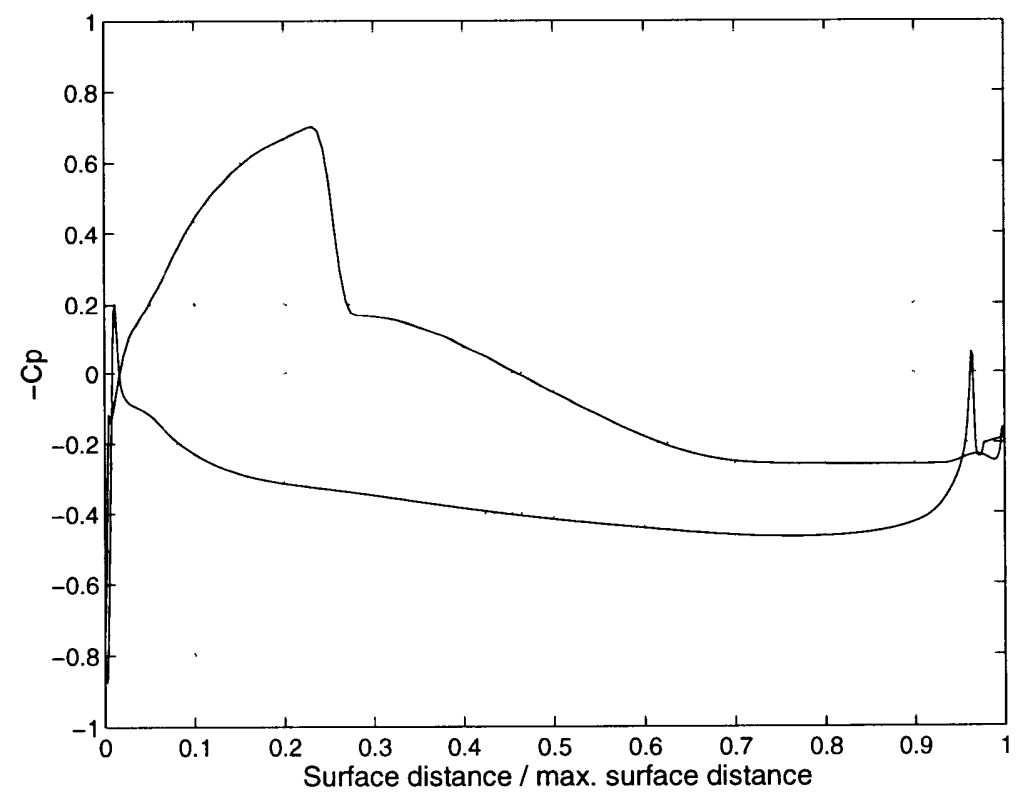

Figure A-4: Time averaged blade pressure distribution. $M_{\infty}=0.87$. 

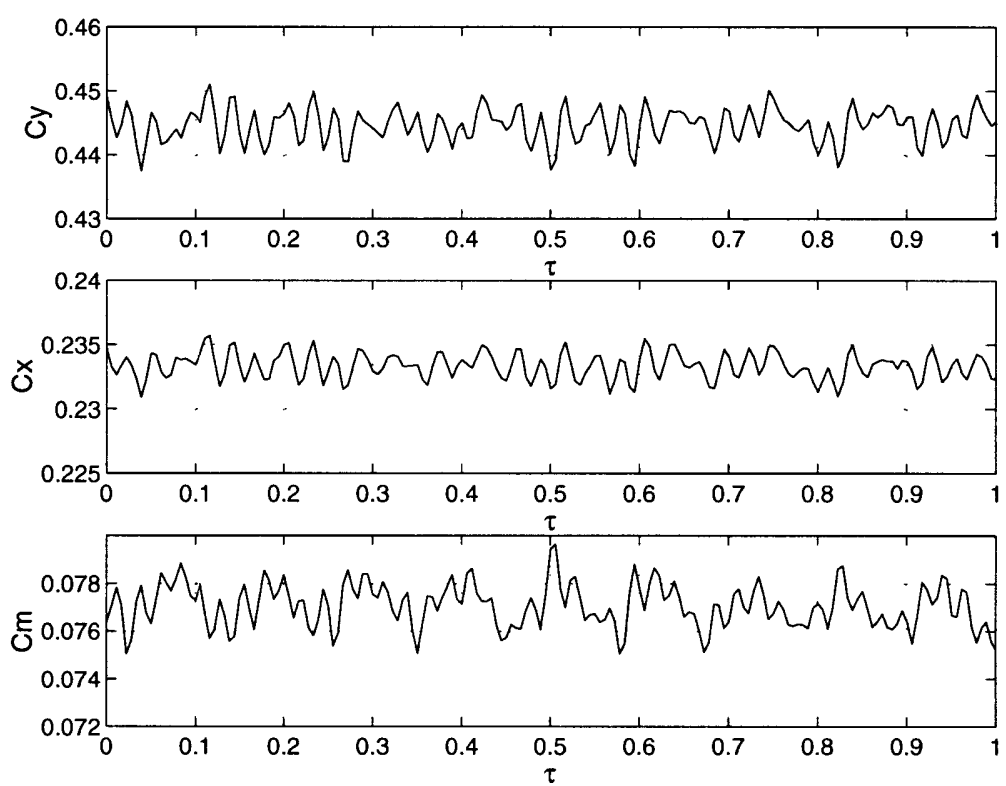

Figure A-5: The force and moment coefficient fluctuation in the baseline solution of Run 1 . $C y, C x$ and $C m$ are the blade azimuthal force, axial force and moment coefficients respectively. $\tau=$ convective time scale. $M_{\infty}=0.15$.
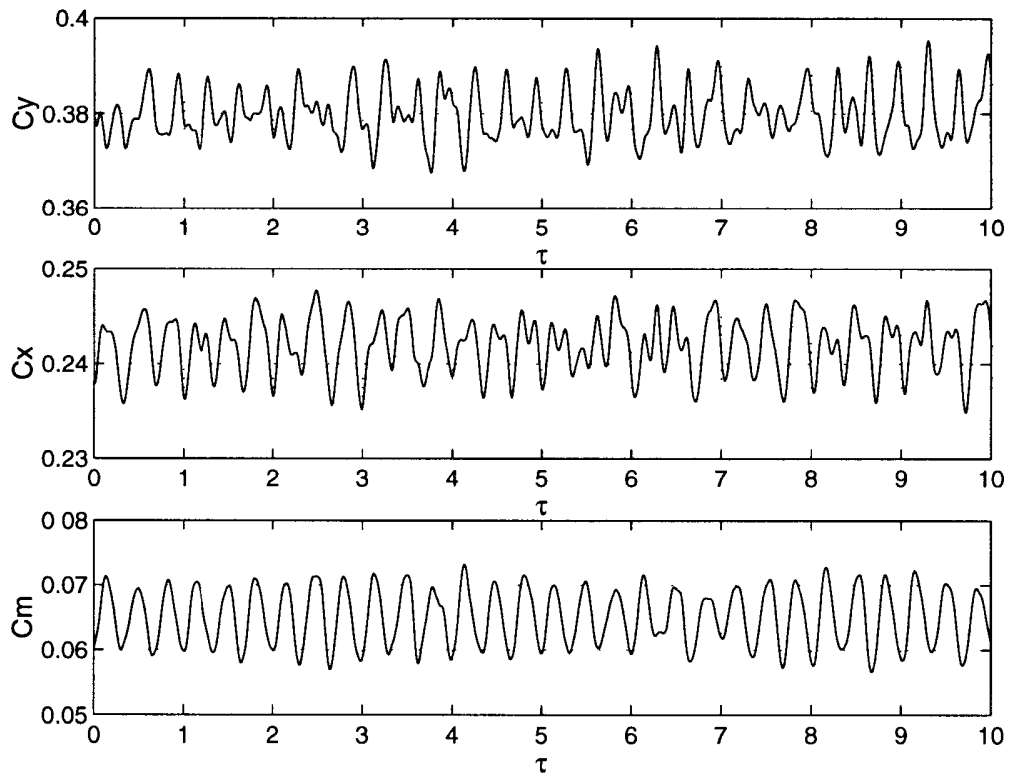

Figure A-6: The force and moment coefficient fluctuation in the baseline solution of Run 2 . $C y, C x$ and $C m$ are the blade azimuthal force, axial force and moment coefficients respectively. $\tau=$ convective time scale. $M_{\infty}=0.53$. 

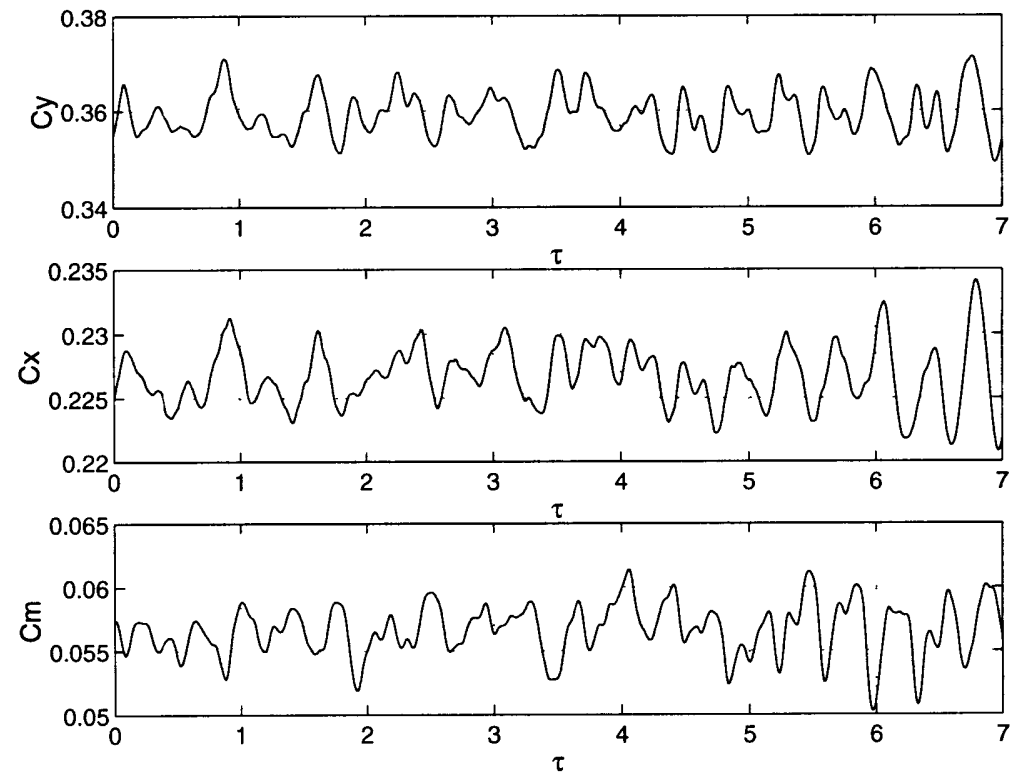

Figure A-7: The force and moment coefficient fluctuation in the baseline solution of Run 3 . $C y, C x$ and $C m$ are the blade azimuthal force, axial force and moment coefficients respectively. $\tau=$ convective time scale. $M_{\infty}=0.63$.
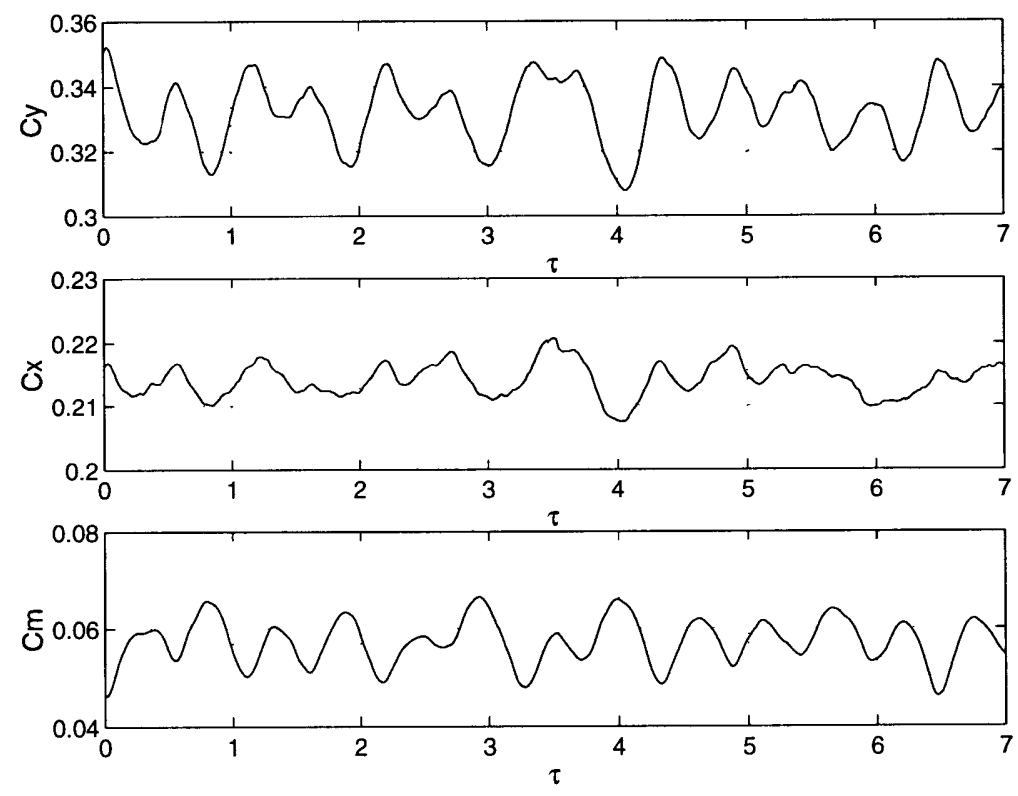

Figure A-8: The force and moment coefficient fluctuation in the baseline solution of Run 4 . $C y, C x$ and $C m$ are the blade azimuthal force, axial force and moment coefficients respectively. $\tau=$ convective time scale. $M_{\infty}=0.87$. 

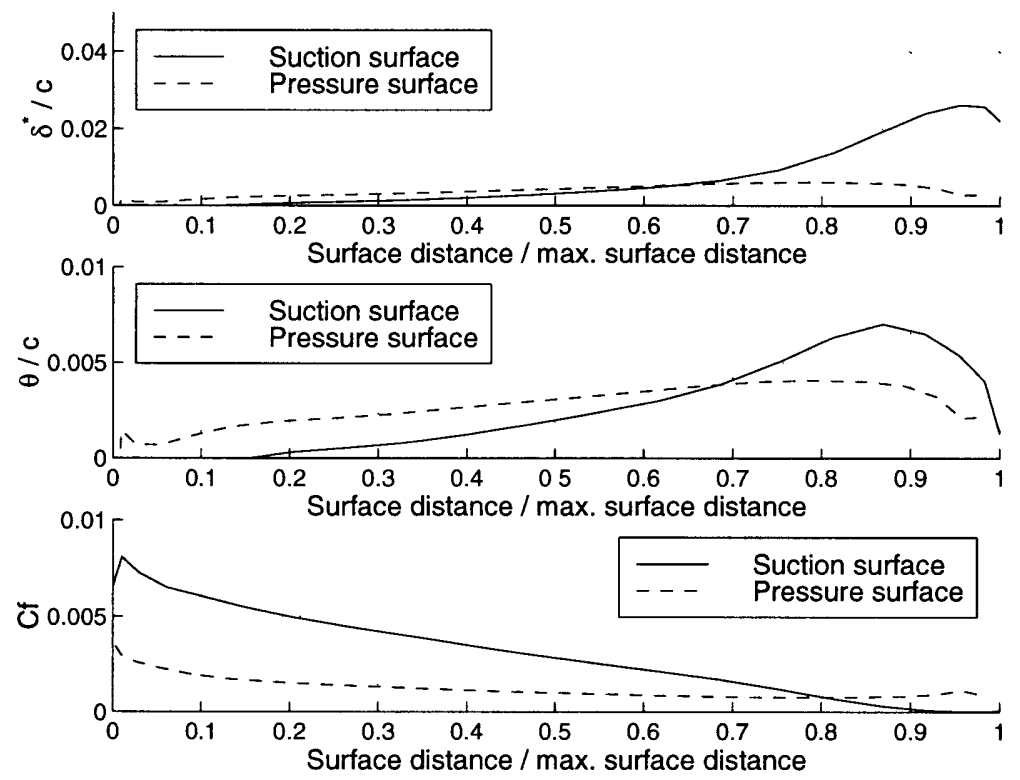

Figure A-9: Time averaged boundary layer properties. $M_{\infty}=0.15$.
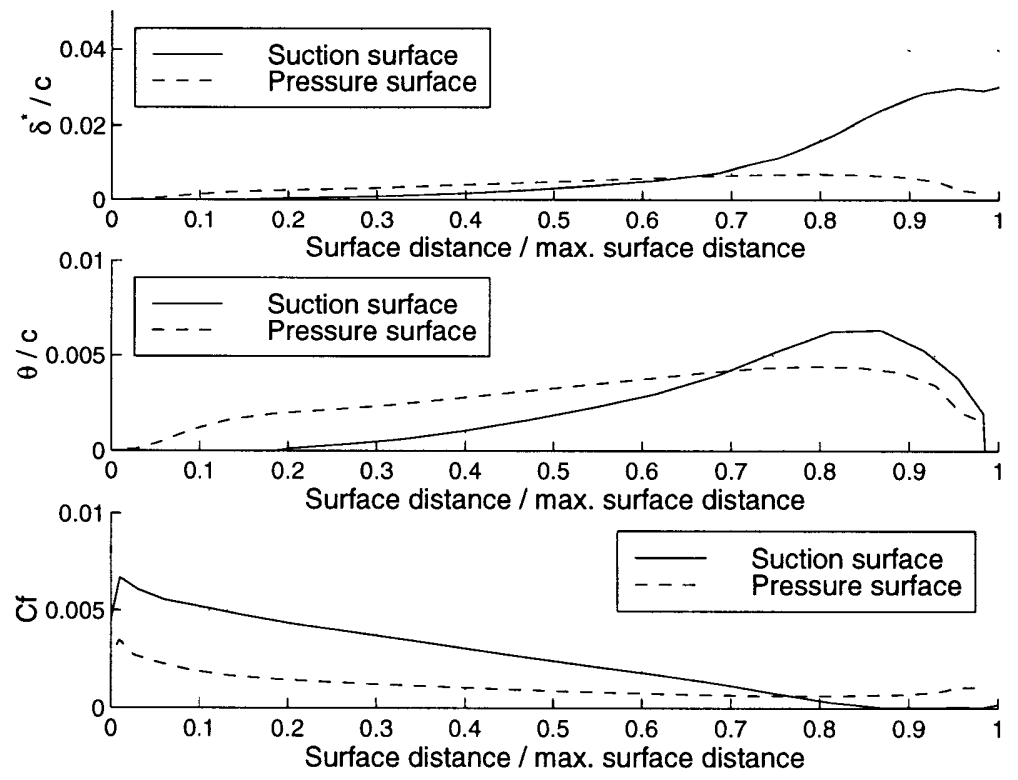

Figure A-10: Time averaged boundary layer properties. $M_{\infty}=0.53$. 

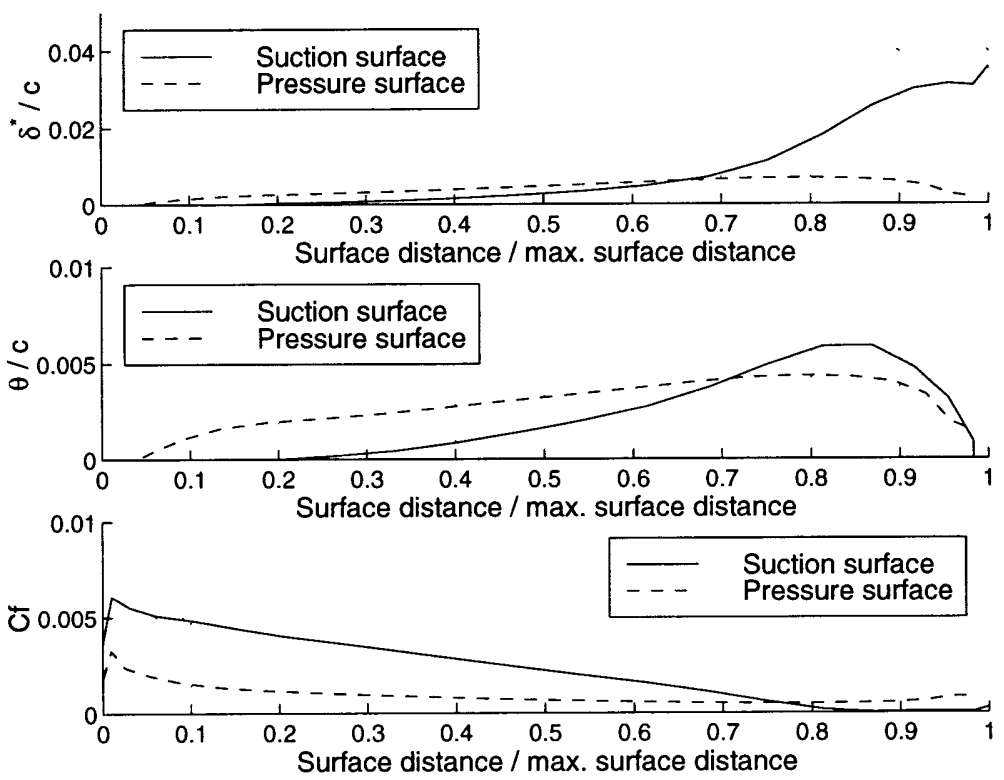

Figure A-11: Time averaged boundary layer properties. $M_{\infty}=0.63$.
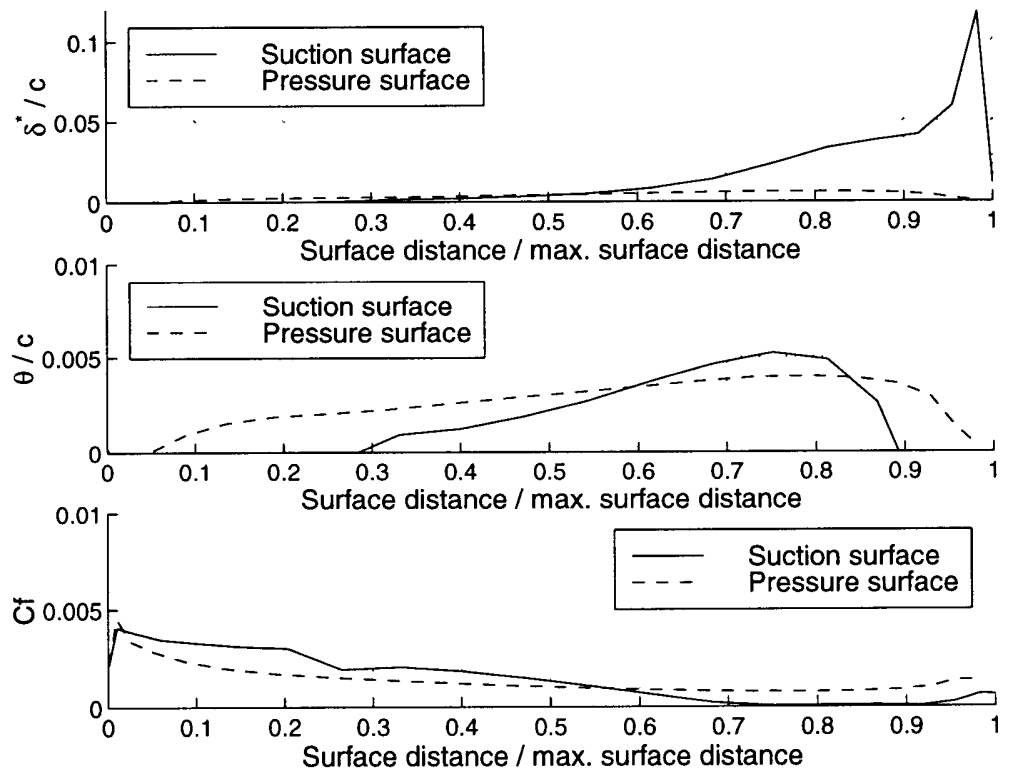

Figure A-12: Time averaged boundary layer properties. $M_{\infty}=0.87$. 


\section{APPENDIX B}

\section{Viscous Flow Force And Moment Fluctuation Profiles}

Plots for the density wake induced (1) azimuthal force coefficient $C y,(2)$ axial force coefficient $C x$ and (3) moment coefficient $C m$ fluctuations are included in this Appendix. Consecutive plots are ordered first according to the density ratio, secondly by the Mach number and thirdly by the wake width.

Please note:

The moment coefficient is defined positive clockwise and is calculated about the blade coordinates $x / c=0.42$ and $y / c=0.29$ (see Figure 2-4). 

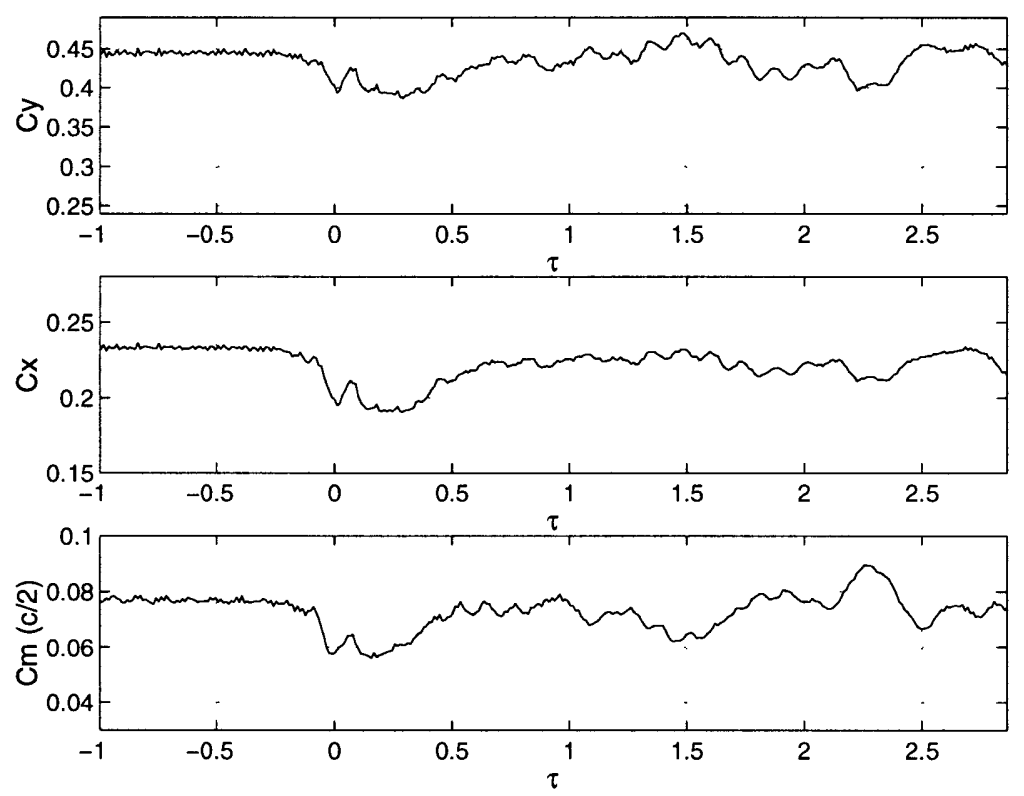

Figure B-1: Fluctuation in blade force and moment coefficients. $w / c=0.1, \rho_{2} / \rho_{1}=0.25$, $M_{\infty}=0.15$.
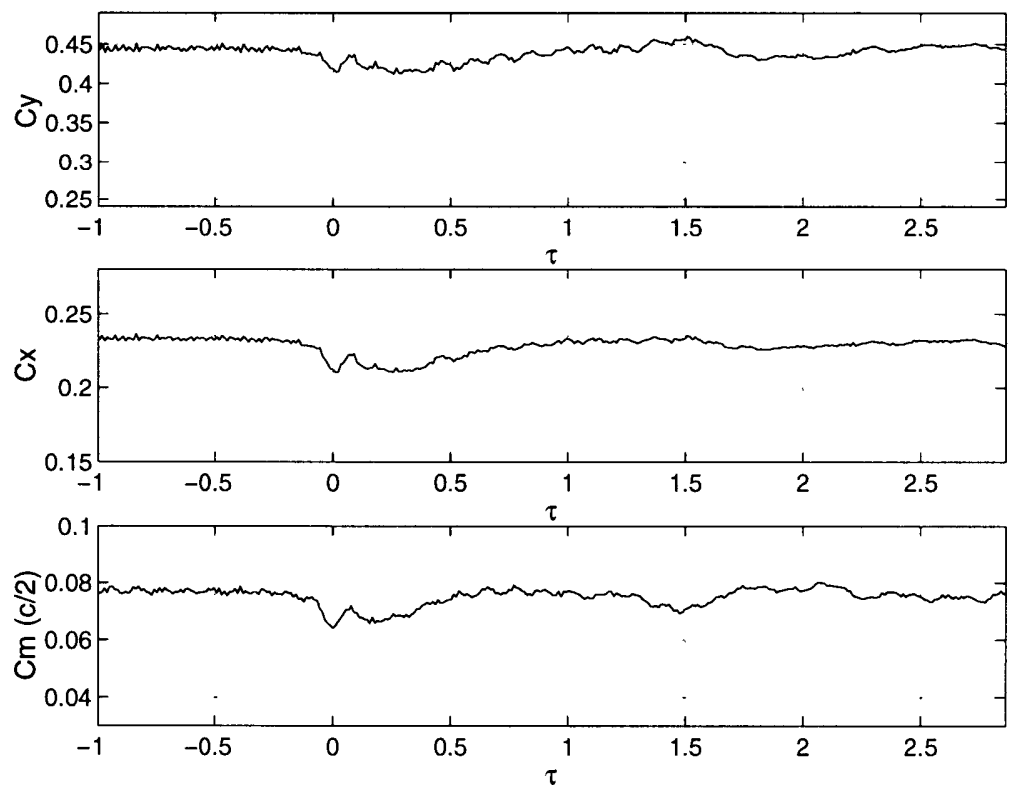

Figure B-2: Fluctuation in blade force and moment coefficients. $w / c=0.1, \rho_{2} / \rho_{1}=0.50$, $M_{\infty}=0.15$. 

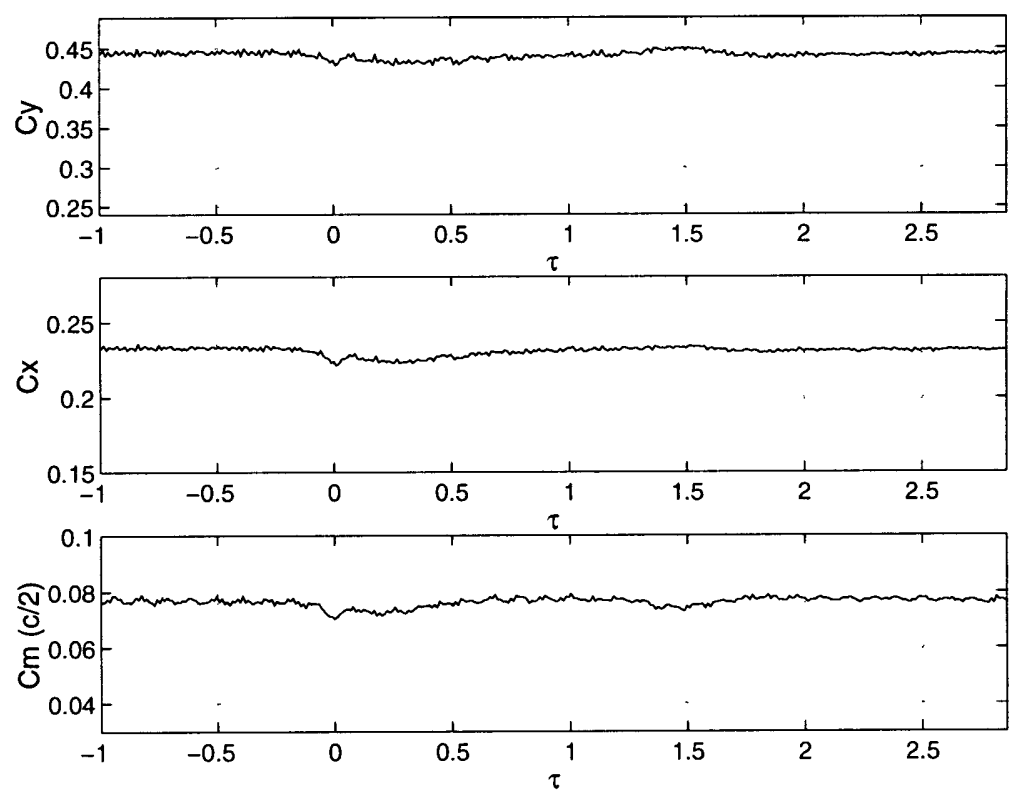

Figure B-3: Fluctuation in blade force and moment coefficients. $w / c=0.1, \rho_{2} / \rho_{1}=0.75$, $M_{\infty}=0.15$.
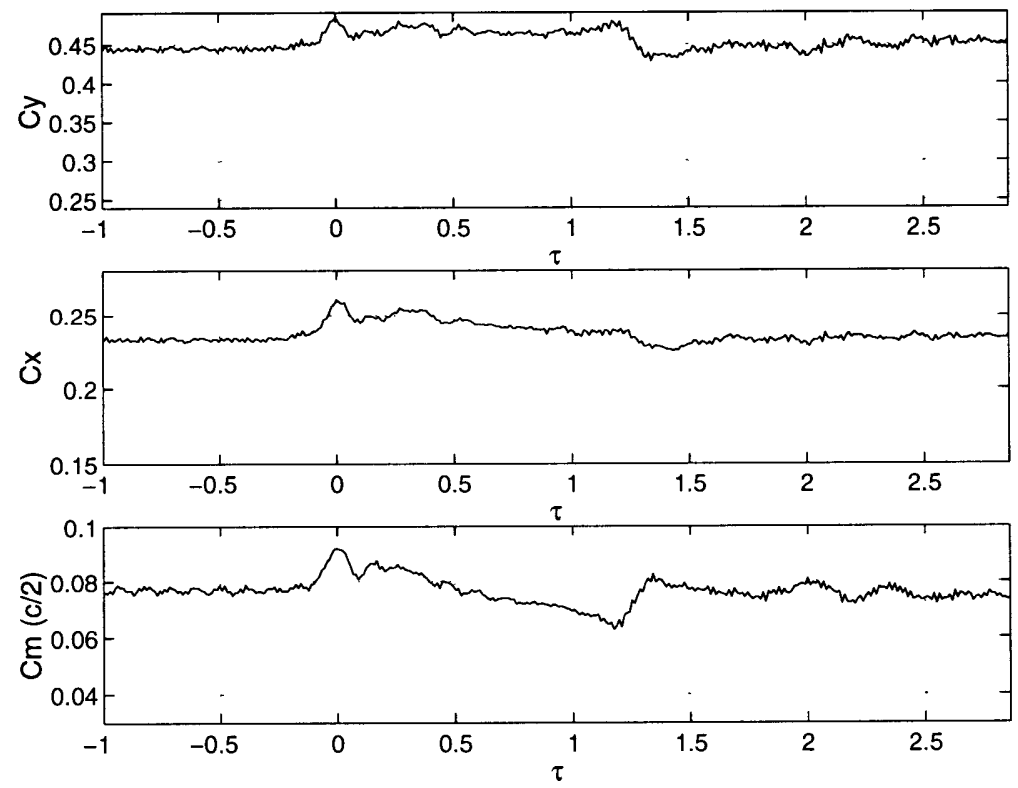

Figure B-4: Fluctuation in blade force and moment coefficients. $w / c=0.1, \rho_{2} / \rho_{1}=2.00$, $M_{\infty}=0.15$. 

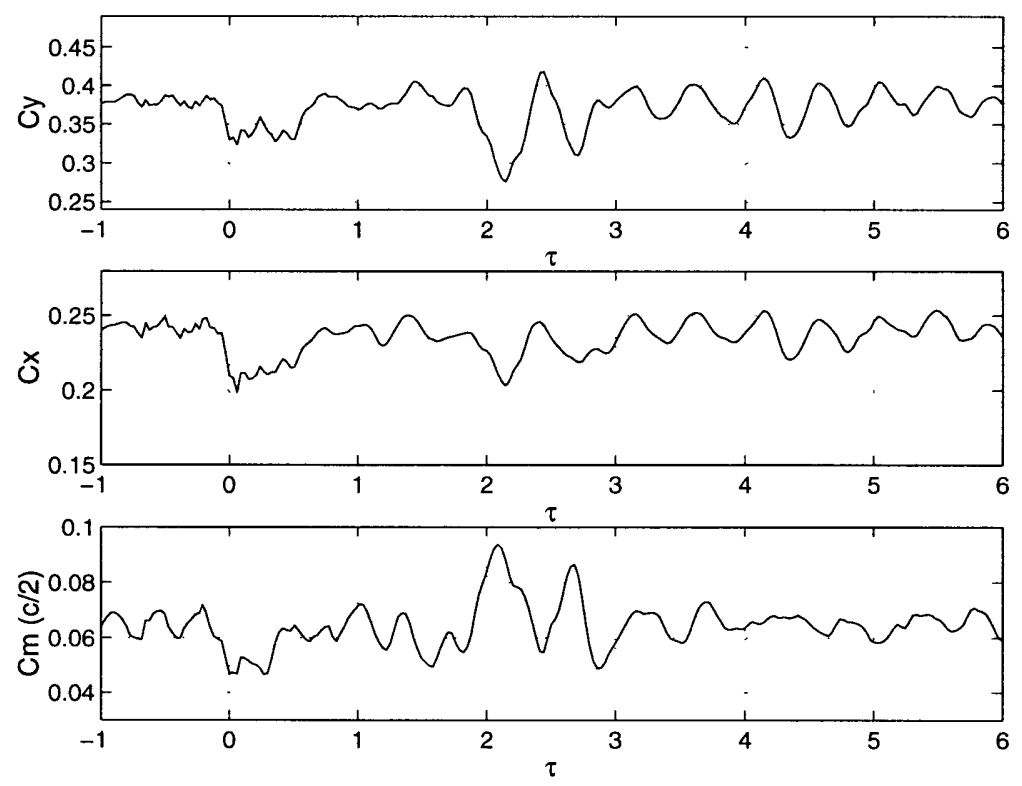

Figure B-5: Fluctuation in blade force and moment coefficients. $w / c=0.1, \rho_{2} / \rho_{1}=0.25$, $M_{\infty}=0.53$.
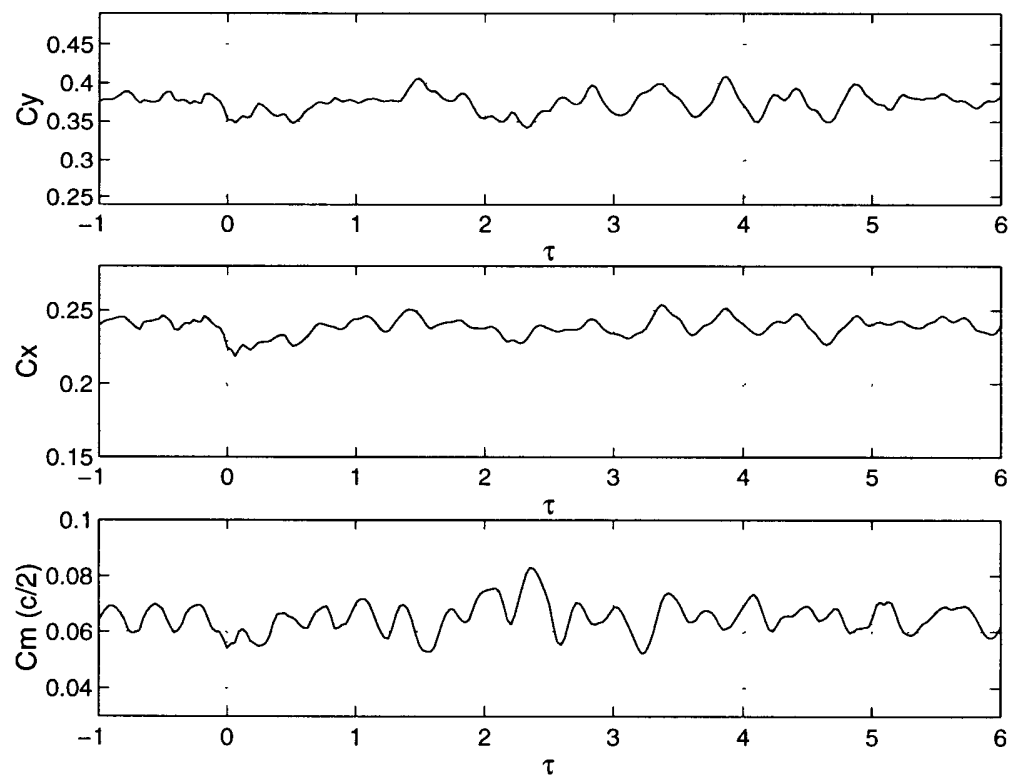

Figure B-6: Fluctuation in blade force and moment coefficients. $w / c=0.1, \rho_{2} / \rho_{1}=0.50$, $M_{\infty}=0.53$. 

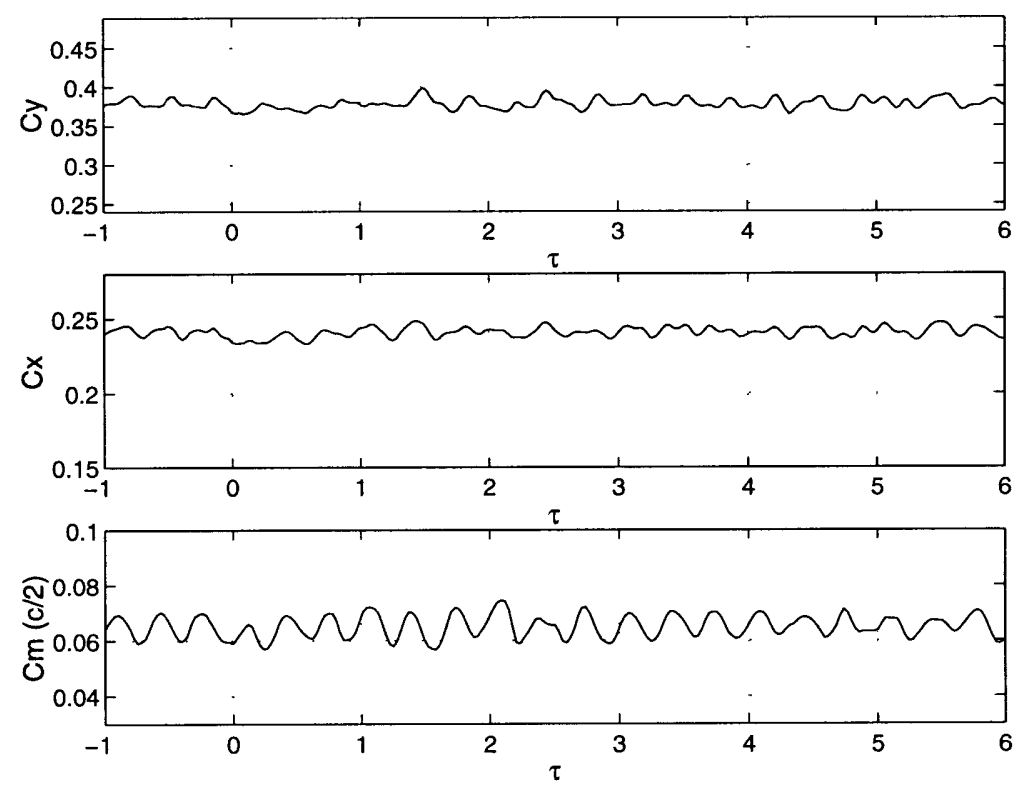

Figure B-7: Fluctuation in blade force and moment coefficients. $w / c=0.1, \rho_{2} / \rho_{1}=0.75$, $M_{\infty}=0.53$.
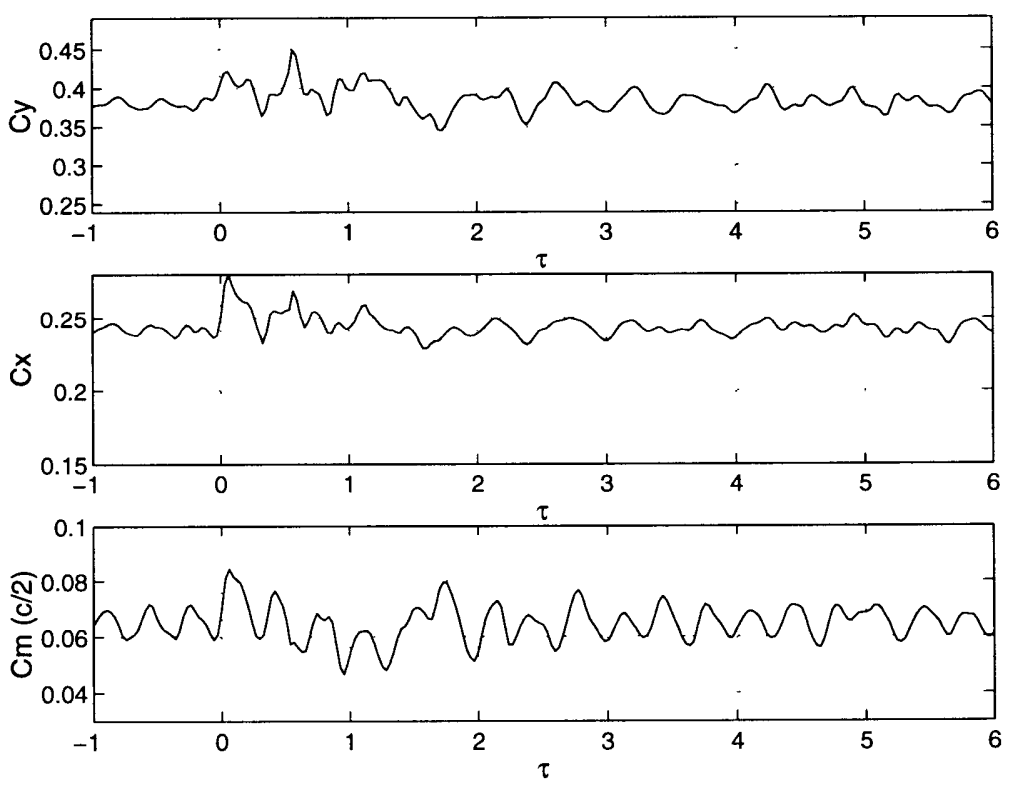

Figure B-8: Fluctuation in blade force and moment coefficients. $w / c=0.1, \rho_{2} / \rho_{1}=2.00$, $M_{\infty}=0.53$. 

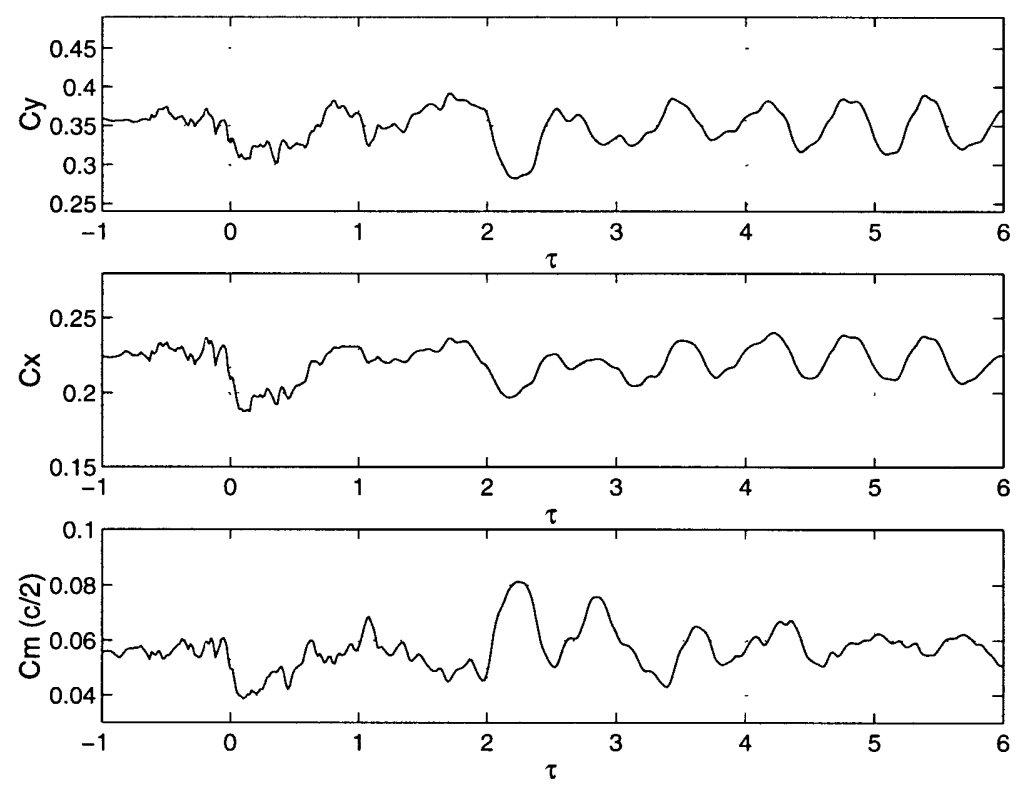

Figure B-9: Fluctuation in blade force and moment coefficients. $w / c=0.1, \rho_{2} / \rho_{1}=0.25$, $M_{\infty}=0.63$.
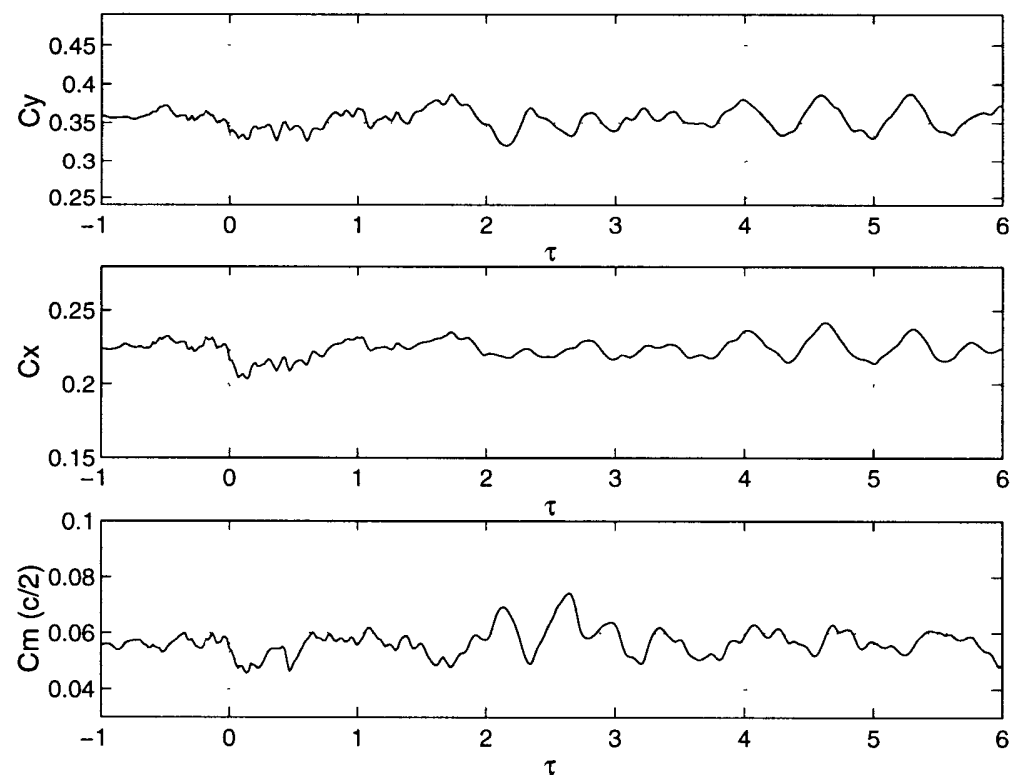

Figure B-10: Fluctuation in blade force and moment coefficients. $w / c=0.1, \rho_{2} / \rho_{1}=0.50$, $M_{\infty}=0.63$. 

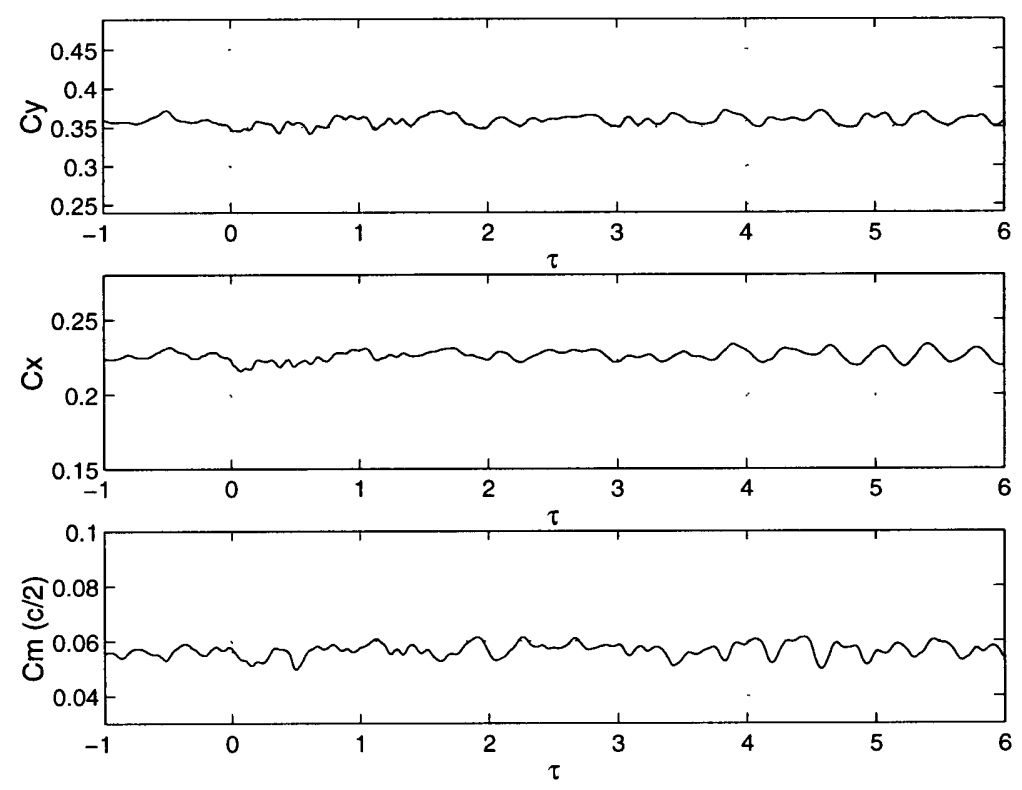

Figure B-11: Fluctuation in blade force and moment coefficients. $w / c=0.1, \rho_{2} / \rho_{1}=0.75$, $M_{\infty}=0.63$.
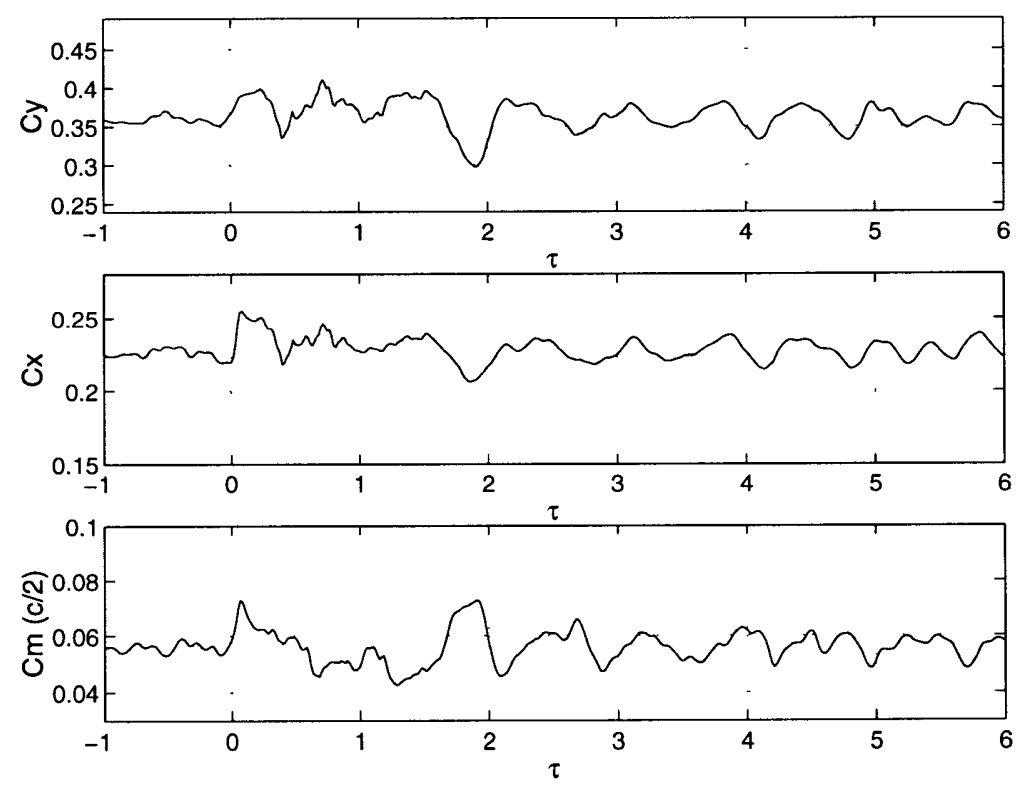

Figure B-12: Fluctuation in blade force and moment coefficients. $w / c=0.1, \rho_{2} / \rho_{1}=2.00$, $M_{\infty}=0.63$. 

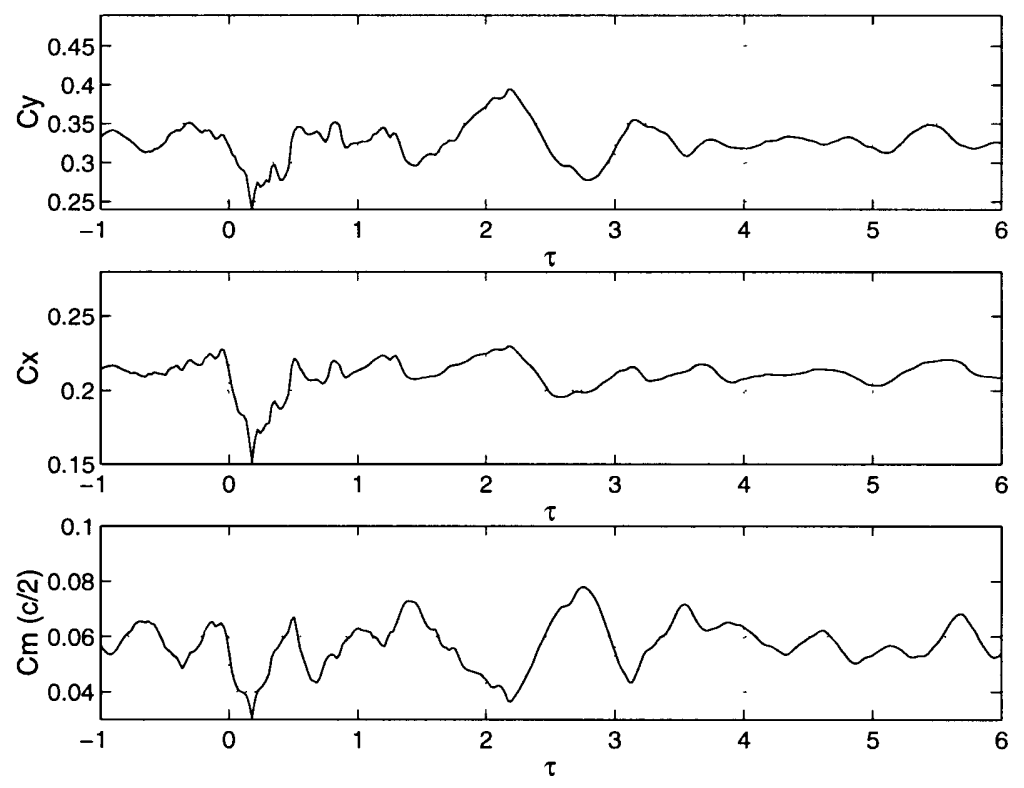

Figure B-13: Fluctuation in blade force and moment coefficients. $w / c=0.1, \rho_{2} / \rho_{1}=0.25$, $M_{\infty}=0.87$.
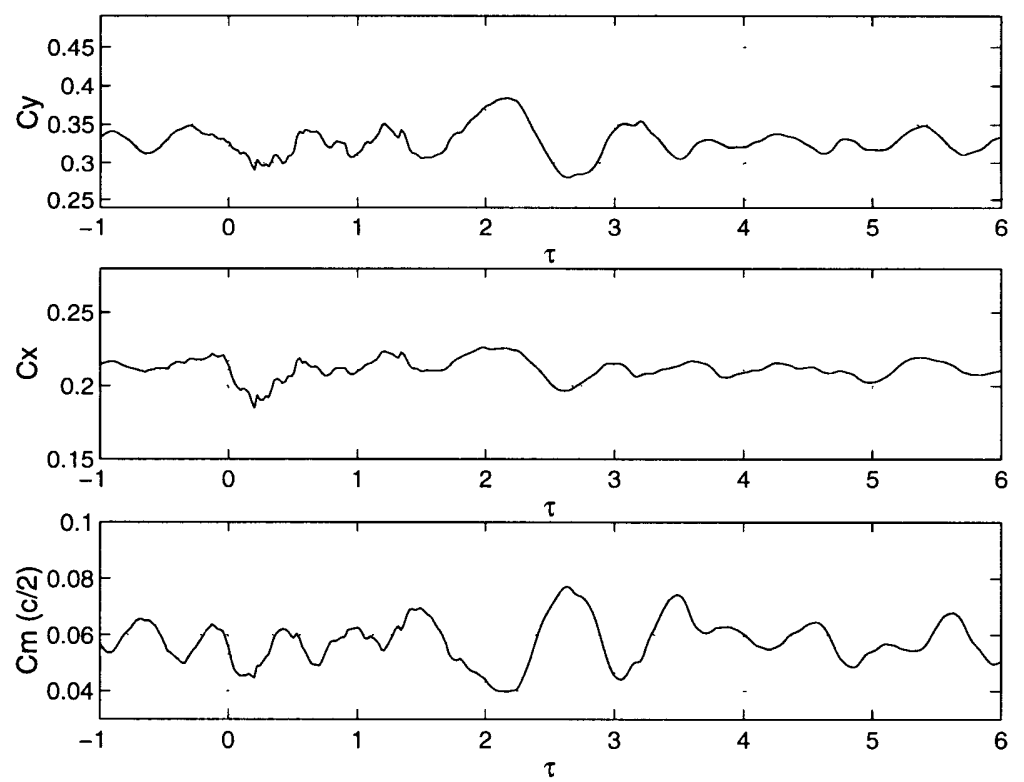

Figure B-14: Fluctuation in blade force and moment coefficients. $w / c=0.1, \rho_{2} / \rho_{1}=0.50$, $M_{\infty}=0.87$. 

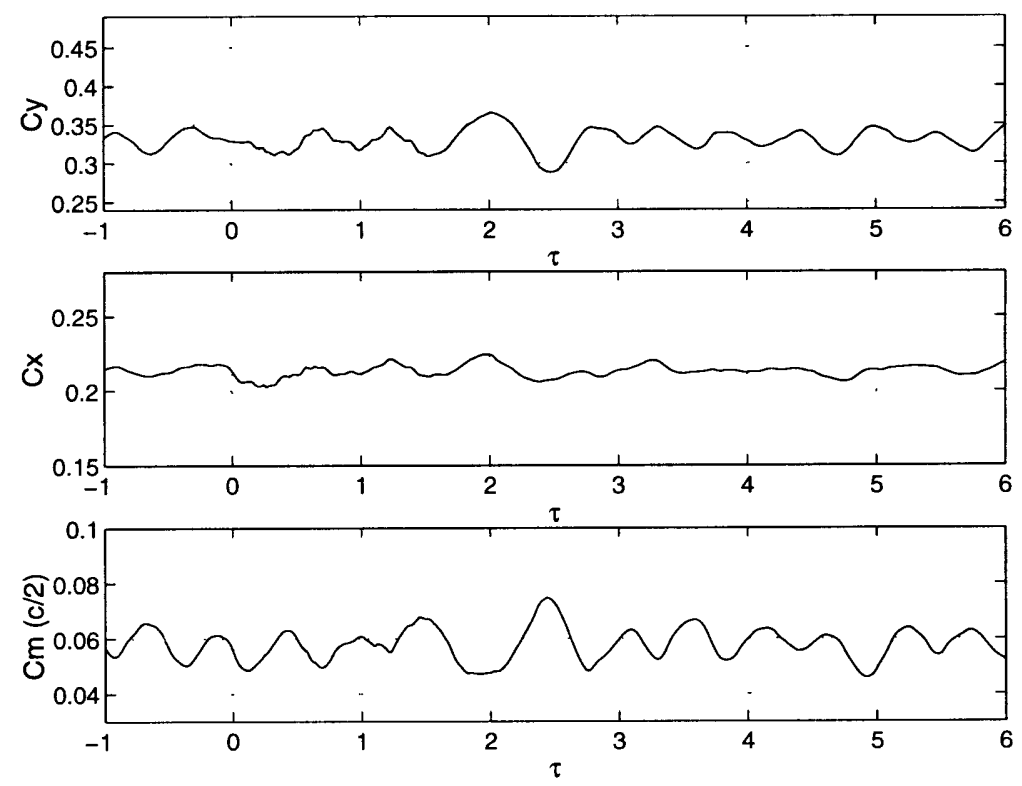

Figure B-15: Fluctuation in blade force and moment coefficients. $w / c=0.1, \rho_{2} / \rho_{1}=0.75$, $M_{\infty}=0.87$.
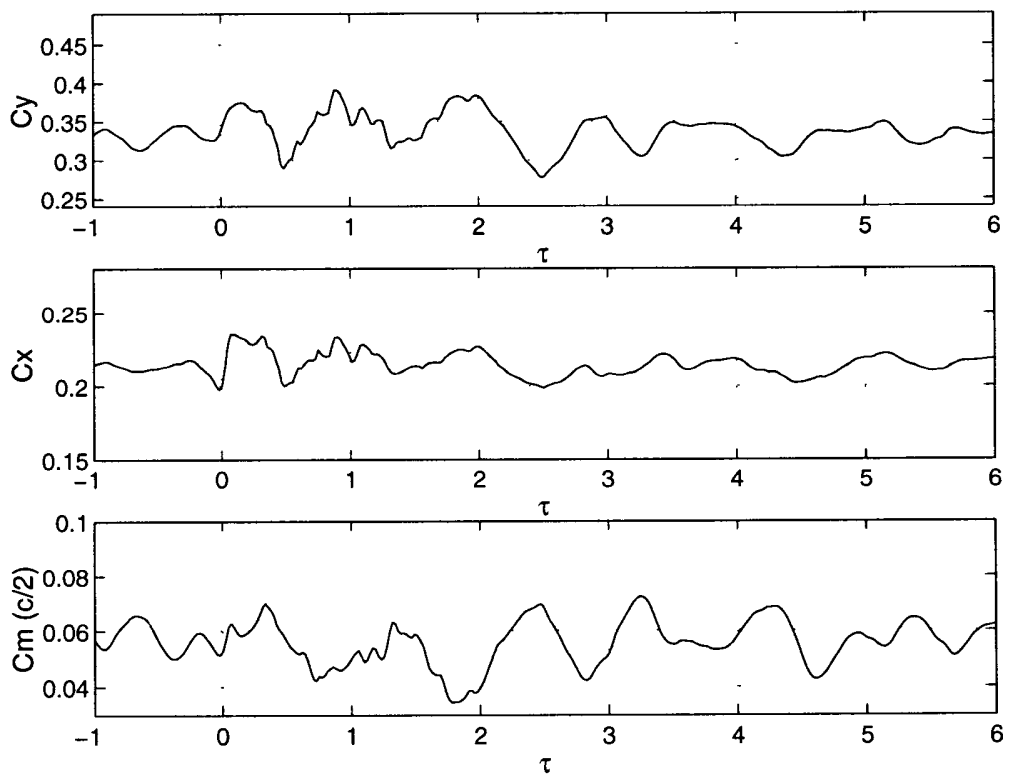

Figure B-16: Fluctuation in blade force and moment coefficients. $w / c=0.1, \rho_{2} / \rho_{1}=2.00$, $M_{\infty}=0.87$. 

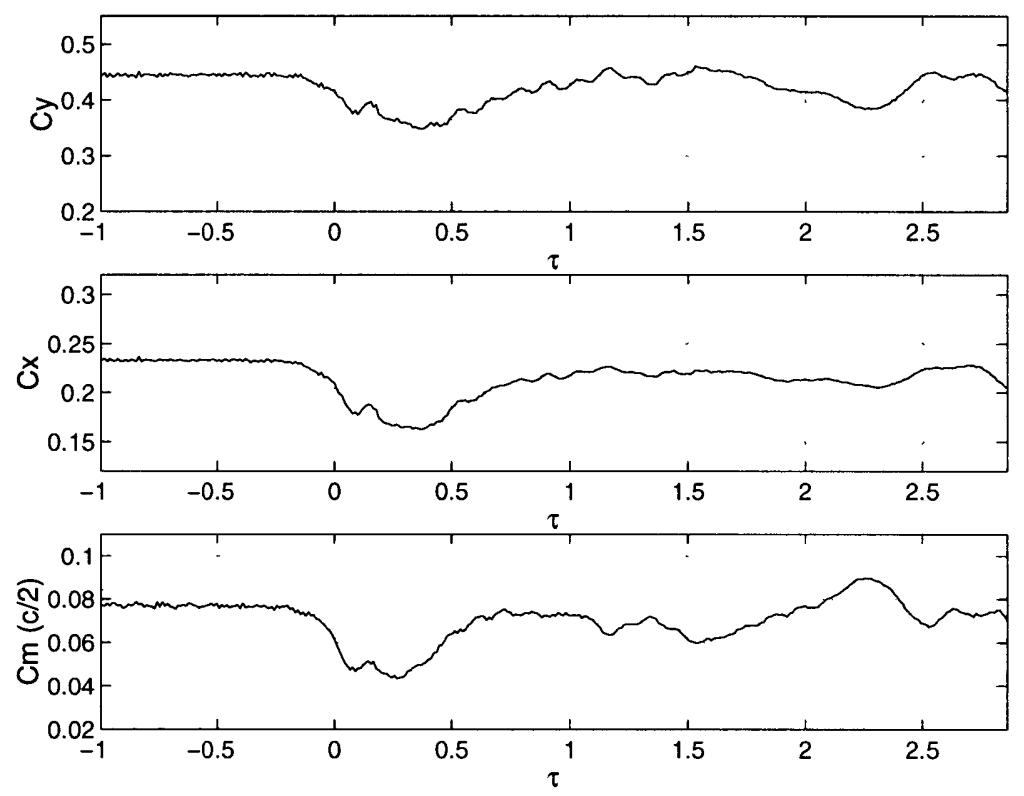

Figure B-17: Fluctuation in blade force and moment coefficients. $w / c=0.2, \rho_{2} / \rho_{1}=0.25$, $M_{\infty}=0.15$.
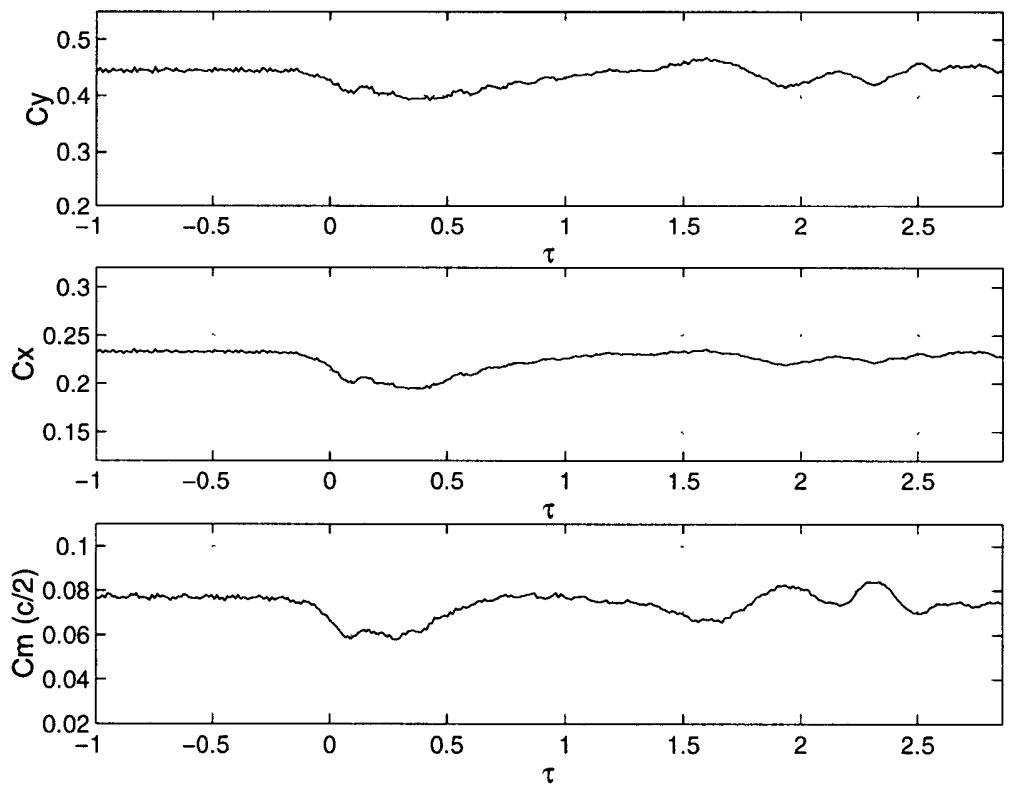

Figure B-18: Fluctuation in blade force and moment coefficients. $w / c=0.2, \rho_{2} / \rho_{1}=0.50$, $M_{\infty}=0.15$. 

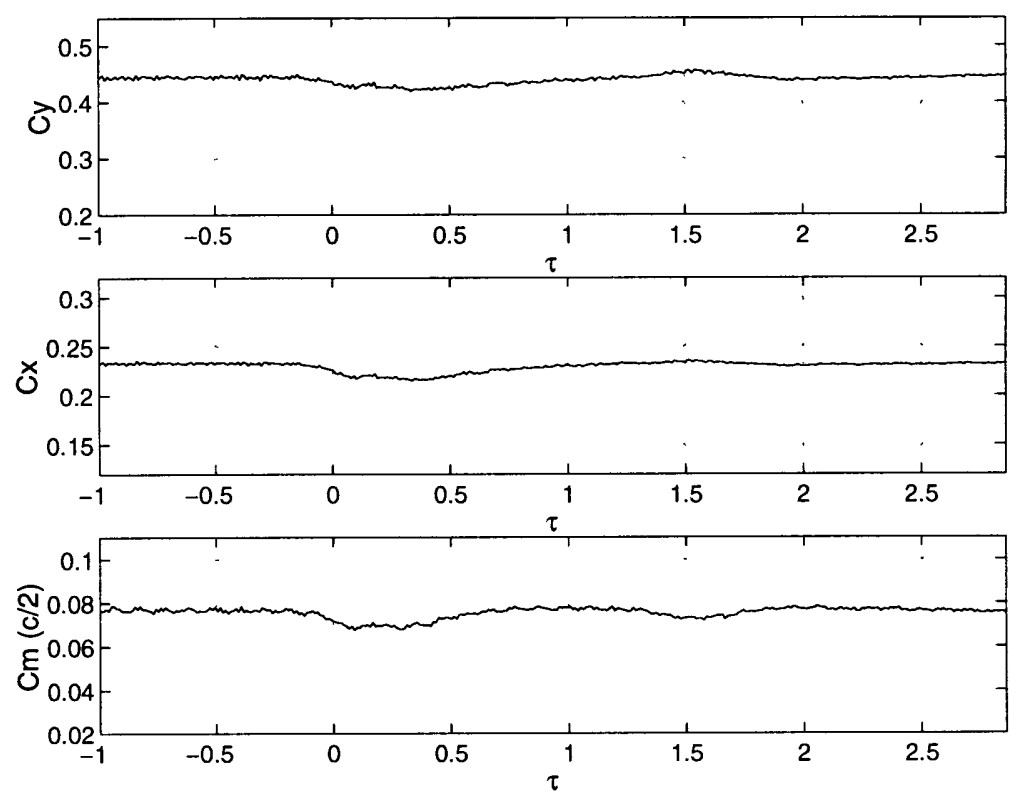

Figure B-19: Fluctuation in blade force and moment coefficients. $w / c=0.2, \rho_{2} / \rho_{1}=0.75$, $M_{\infty}=0.15$.
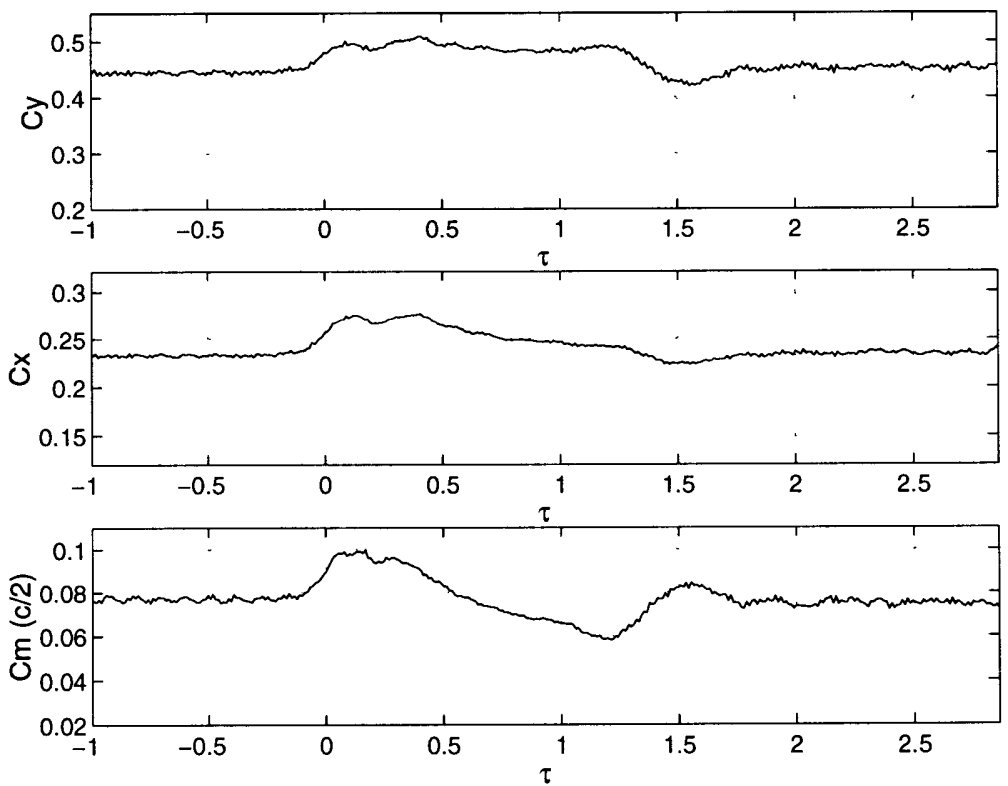

Figure B-20: Fluctuation in blade force and moment coefficients. $w / c=0.2, \rho_{2} / \rho_{1}=2.00$, $M_{\infty}=0.15$. 

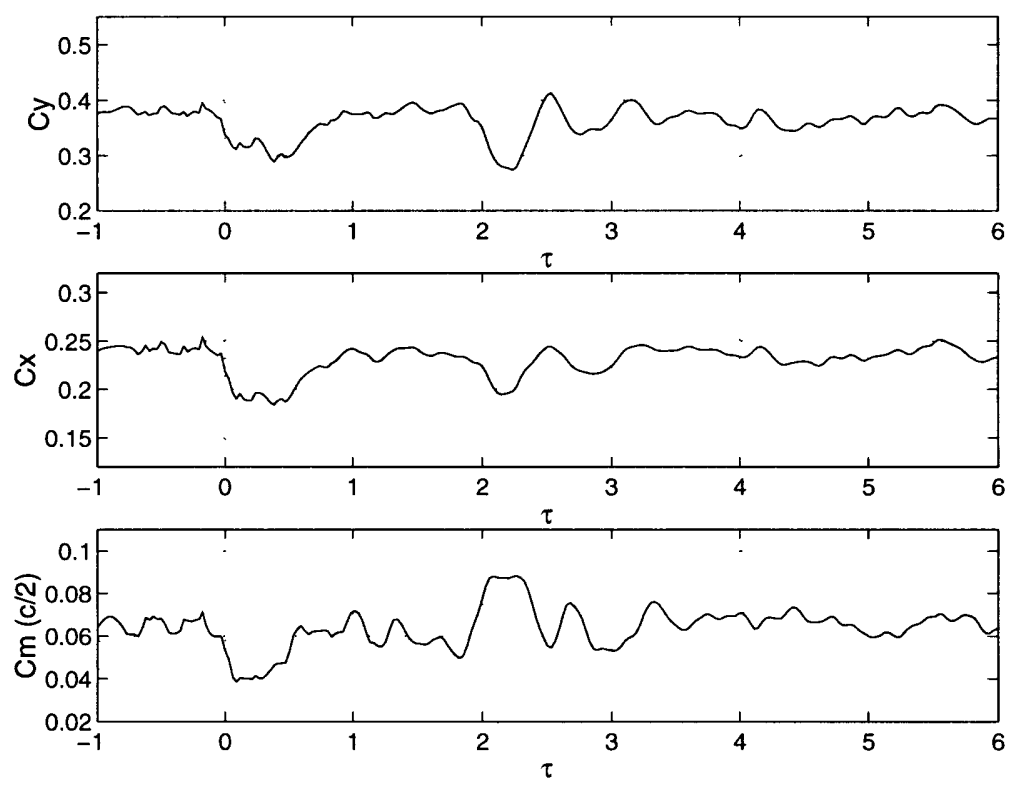

Figure B-21: Fluctuation in blade force and moment coefficients. $w / c=0.2, \rho_{2} / \rho_{1}=0.25$, $M_{\infty}=0.53$.
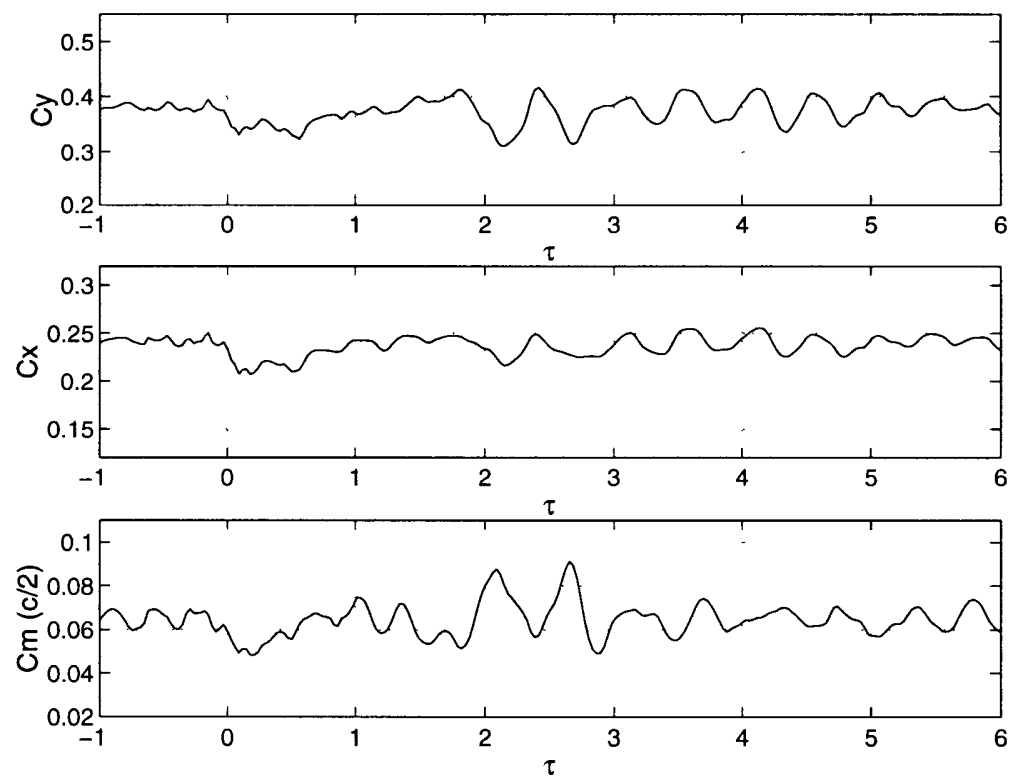

Figure B-22: Fluctuation in blade force and moment coefficients. $w / c=0.2, \rho_{2} / \rho_{1}=0.50$, $M_{\infty}=0.53$. 

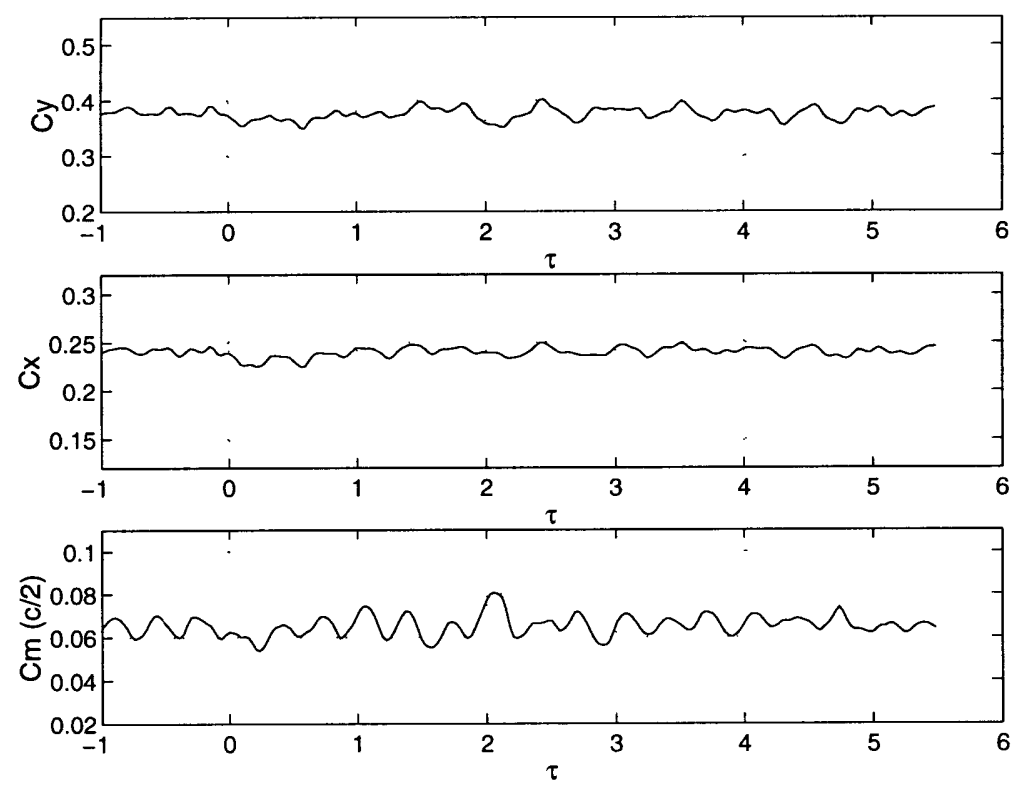

Figure B-23: Fluctuation in blade force and moment coefficients. $w / c=0.2, \rho_{2} / \rho_{1}=0.75$, $M_{\infty}=0.53$.
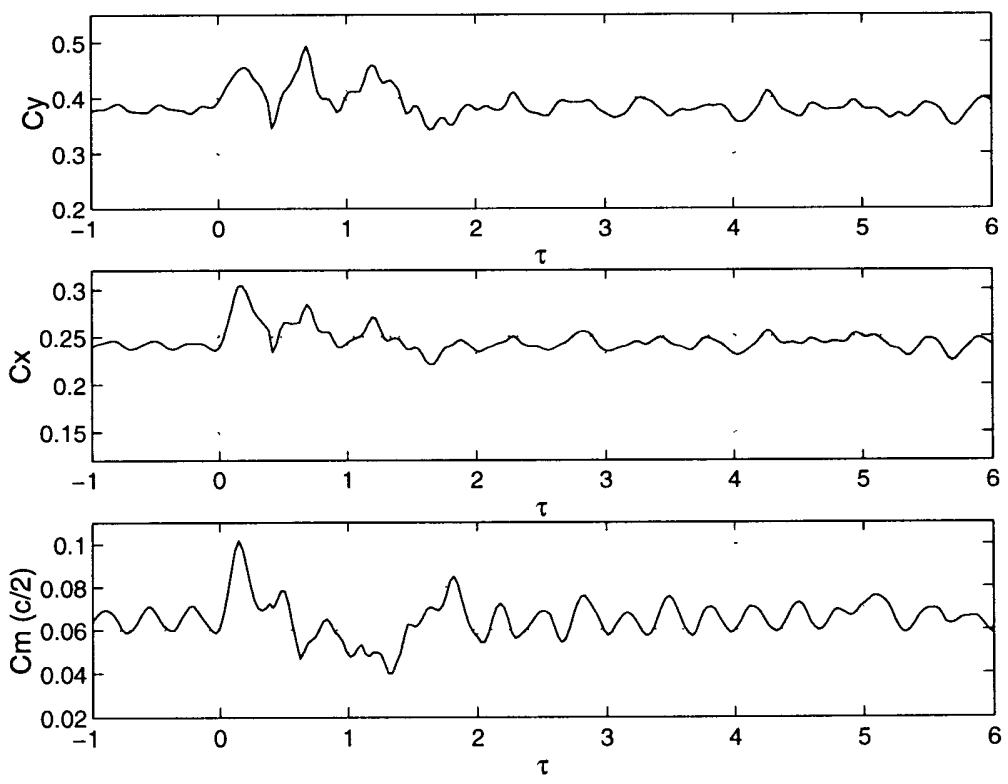

Figure B-24: Fluctuation in blade force and moment coefficients. $w / c=0.2, \rho_{2} / \rho_{1}=2.00$, $M_{\infty}=0.53$. 

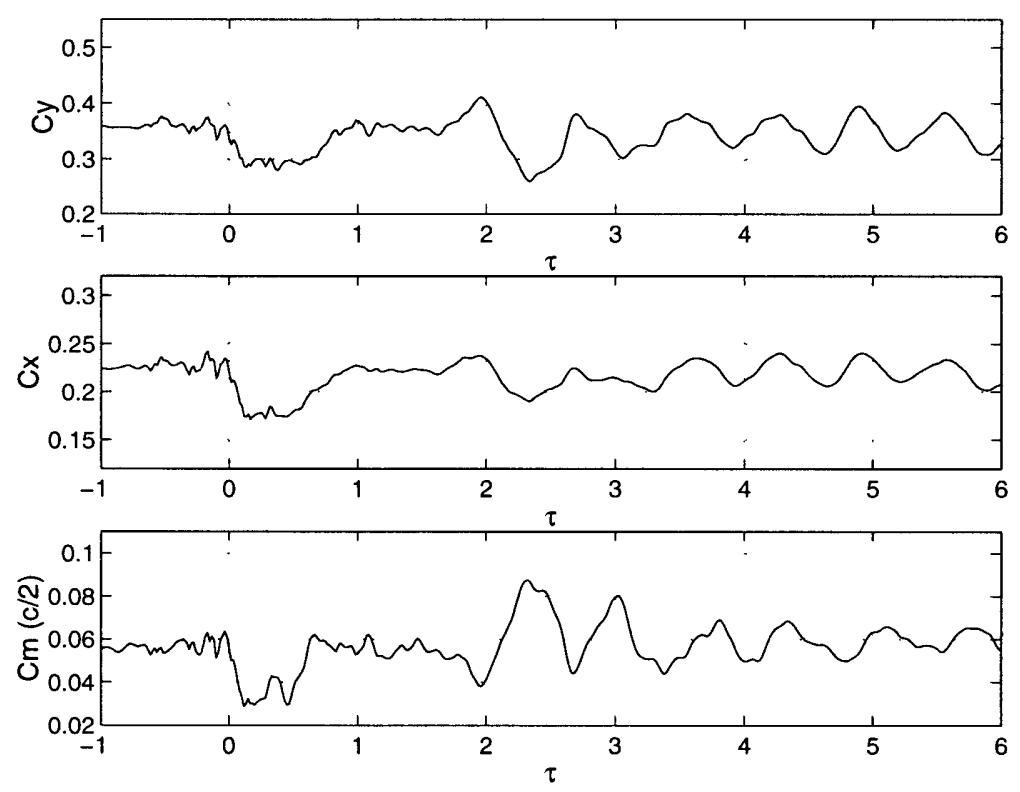

Figure B-25: Fluctuation in blade force and moment coefficients. $w / c=0.2, \rho_{2} / \rho_{1}=0.25$, $M_{\infty}=0.63$.
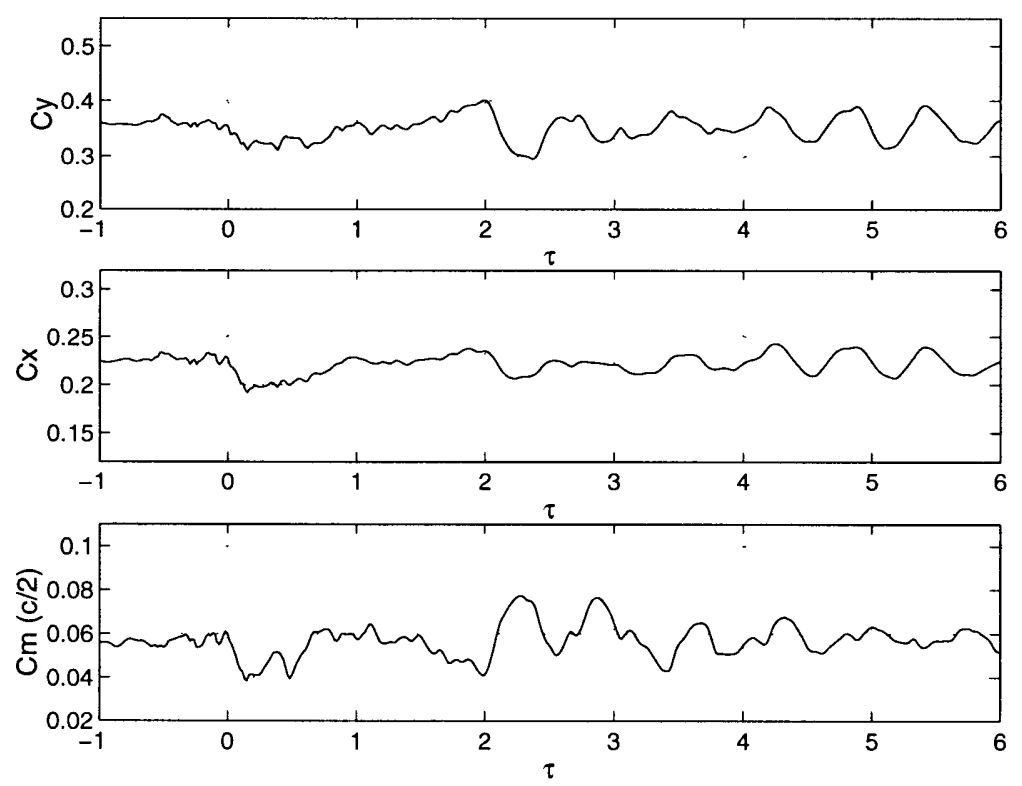

Figure B-26: Fluctuation in blade force and moment coefficients. $w / c=0.2, \rho_{2} / \rho_{1}=0.50$, $M_{\infty}=0.63$. 

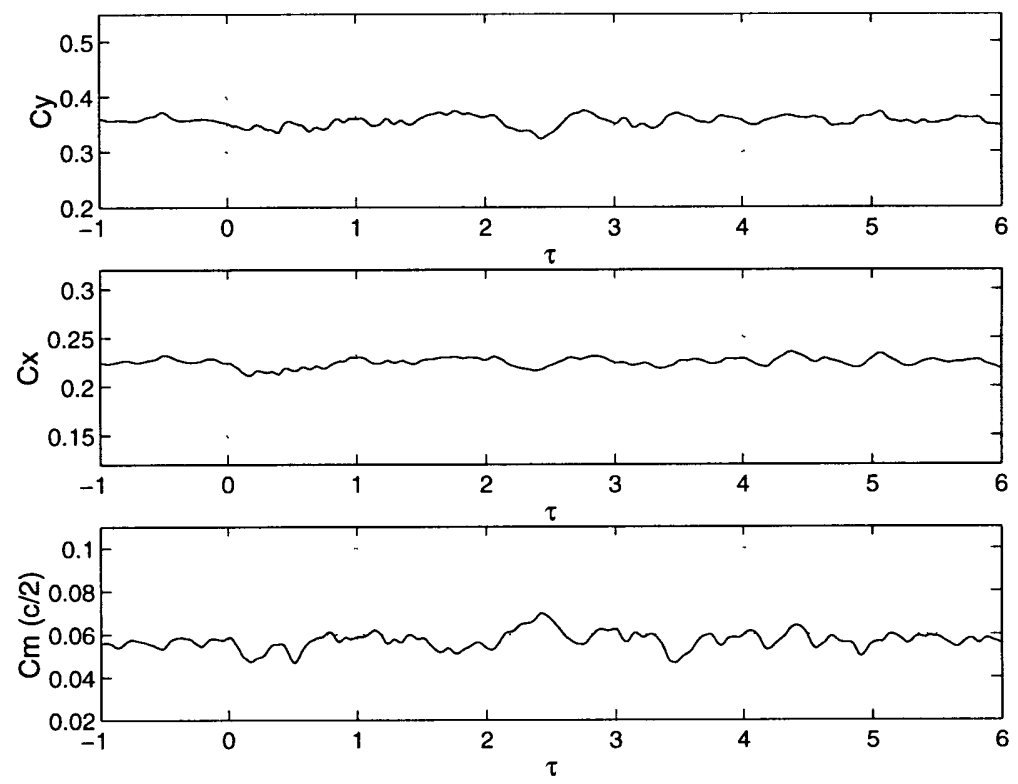

Figure B-27: Fluctuation in blade force and moment coefficients. $w / c=0.2, \rho_{2} / \rho_{1}=0.75$, $M_{\infty}=0.63$.
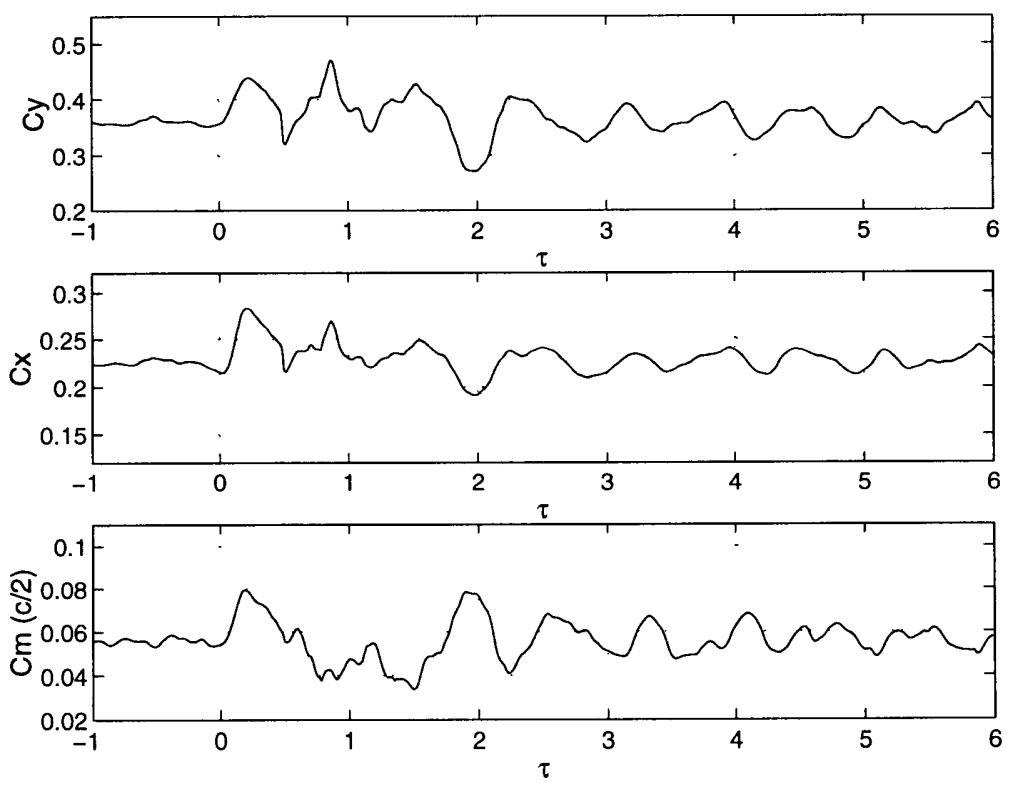

Figure B-28: Fluctuation in blade force and moment coefficients. $w / c=0.2, \rho_{2} / \rho_{1}=2.00$, $M_{\infty}=0.63$. 

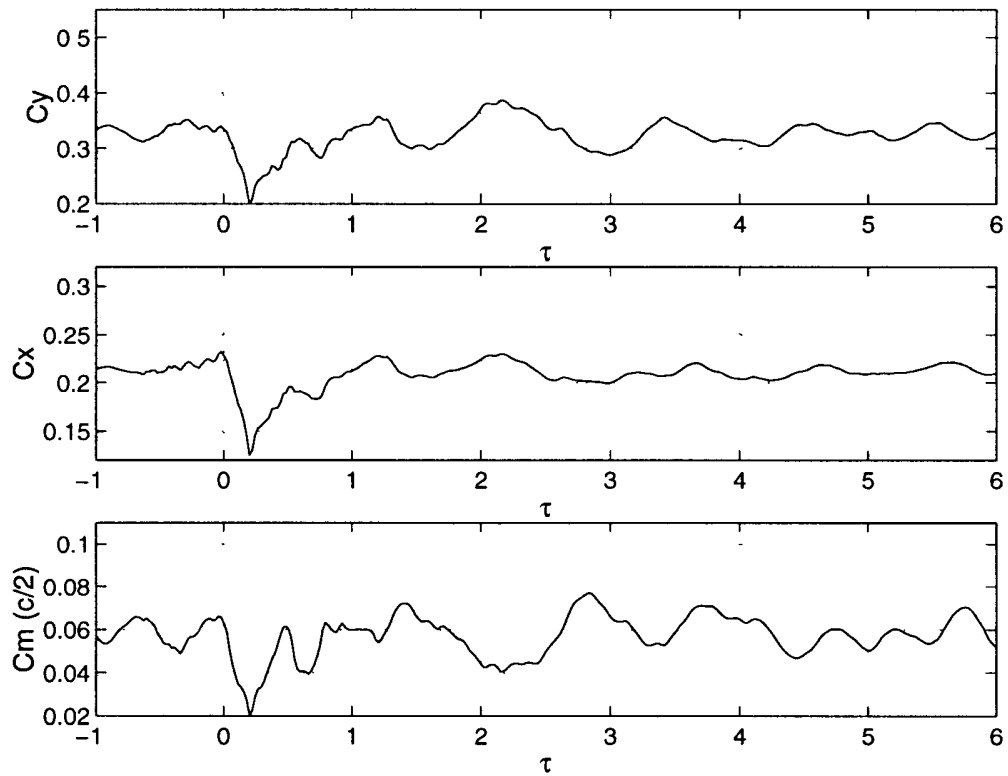

Figure B-29: Fluctuation in blade force and moment coefficients. $w / c=0.2, \rho_{2} / \rho_{1}=0.25$, $M_{\infty}=0.87$.
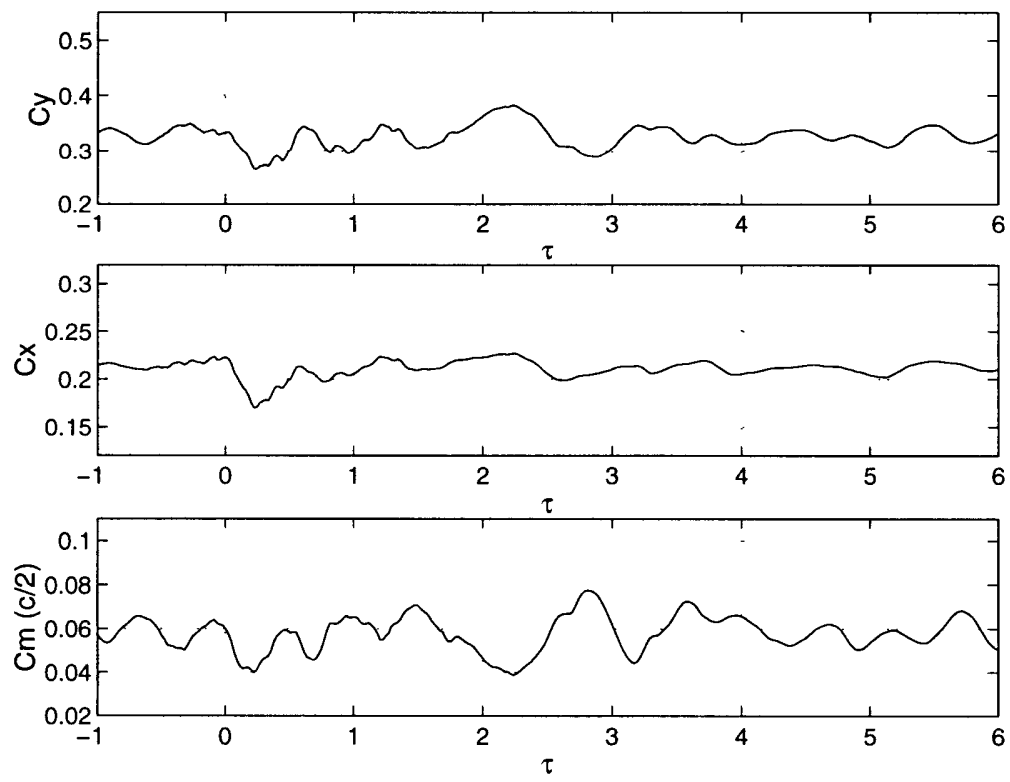

Figure B-30: Fluctuation in blade force and moment coefficients. $w / c=0.2, \rho_{2} / \rho_{1}=0.50$, $M_{\infty}=0.87$. 

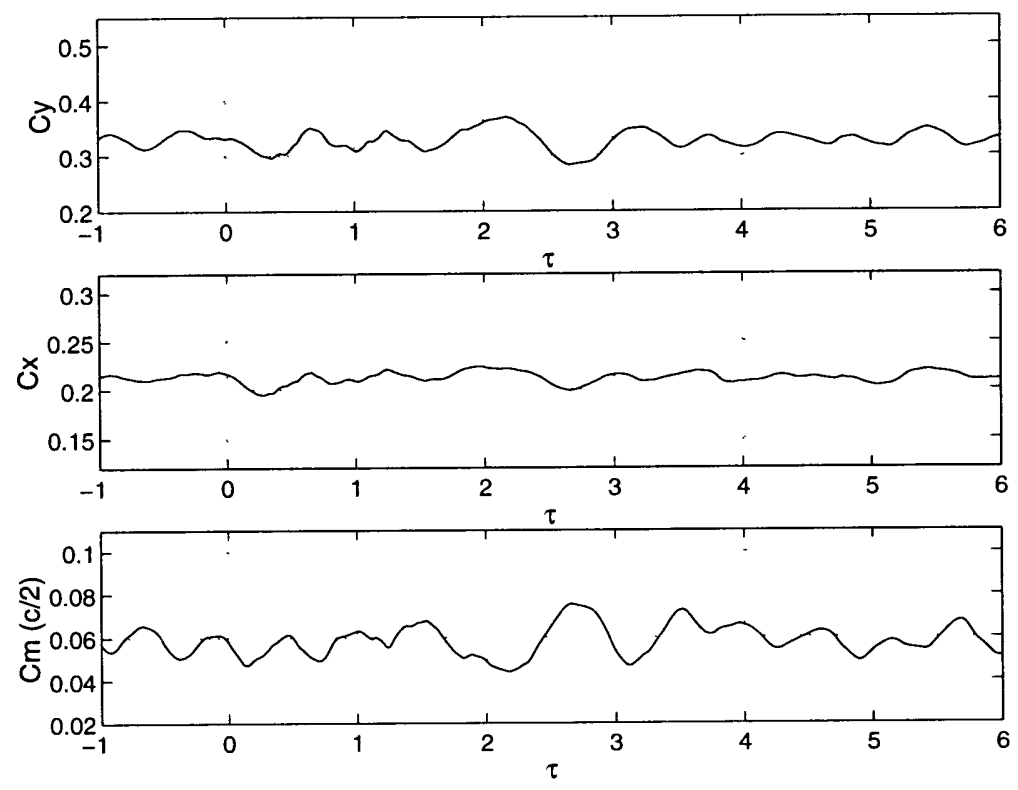

Figure B-31: Fluctuation in blade force and moment coefficients. $w / c=0.2, \rho_{2} / \rho_{1}=0.75$, $M_{\infty}=0.87$.
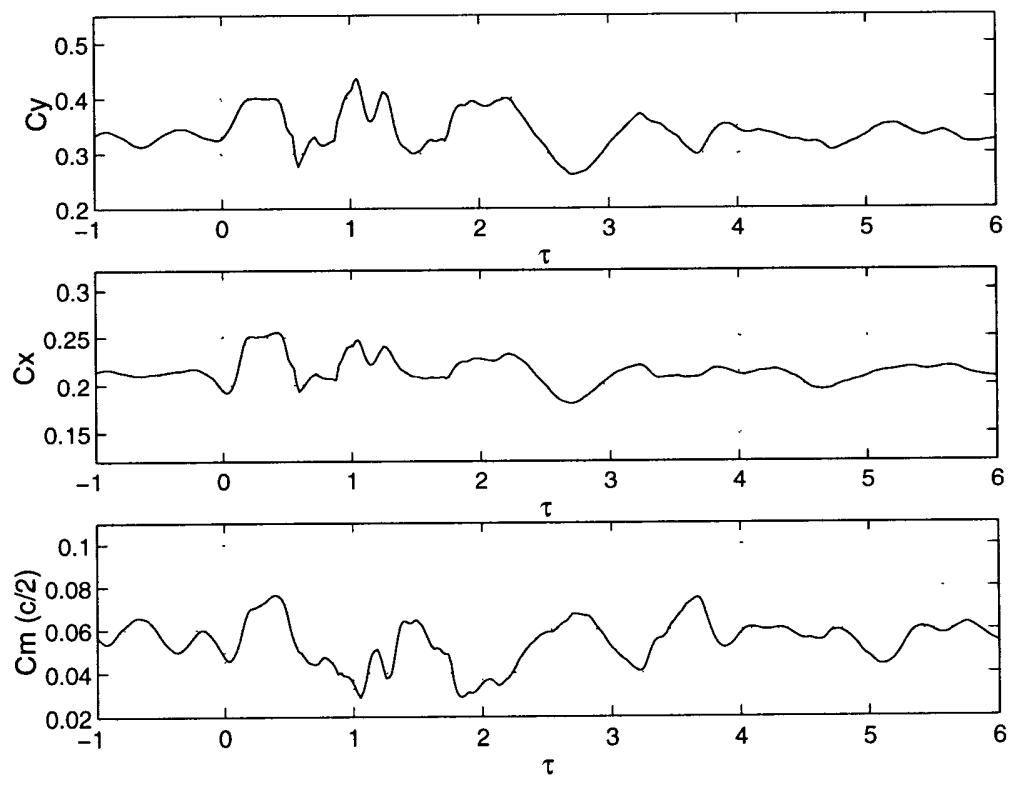

Figure B-32: Fluctuation in blade force and moment coefficients. $w / c=0.2, \rho_{2} / \rho_{1}=2.00$, $M_{\infty}=0.87$. 

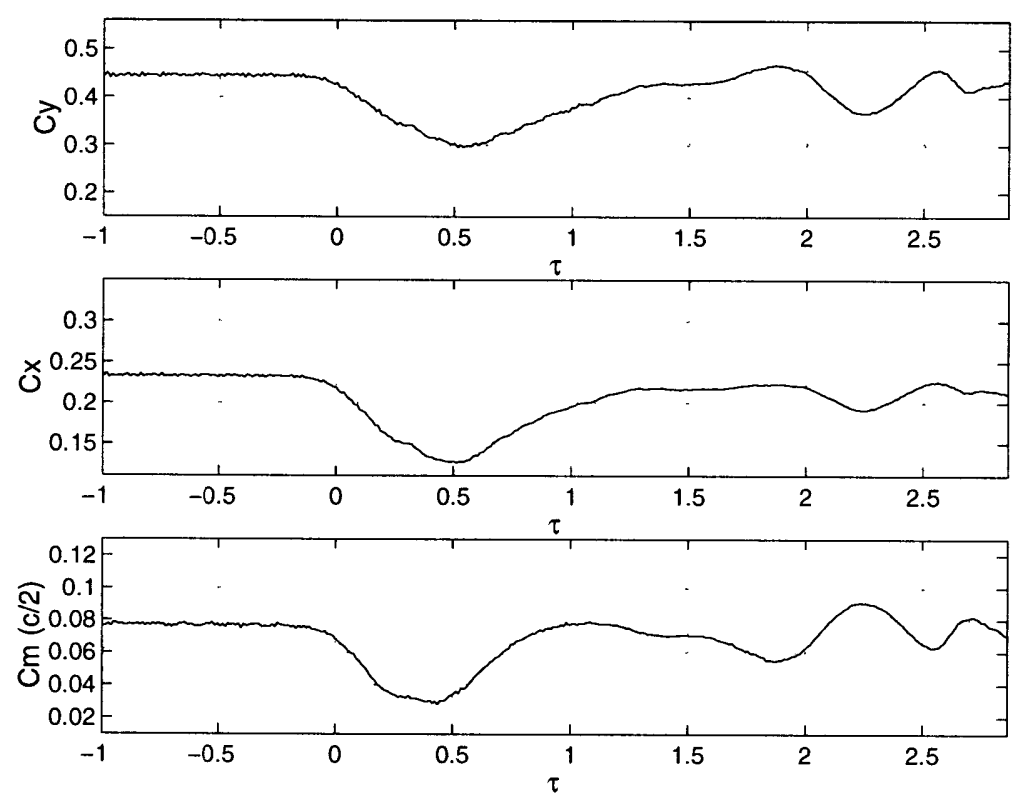

Figure B-33: Fluctuation in blade force and moment coefficients. $w / c=0.4, \rho_{2} / \rho_{1}=0.25$, $M_{\infty}=0.15$.
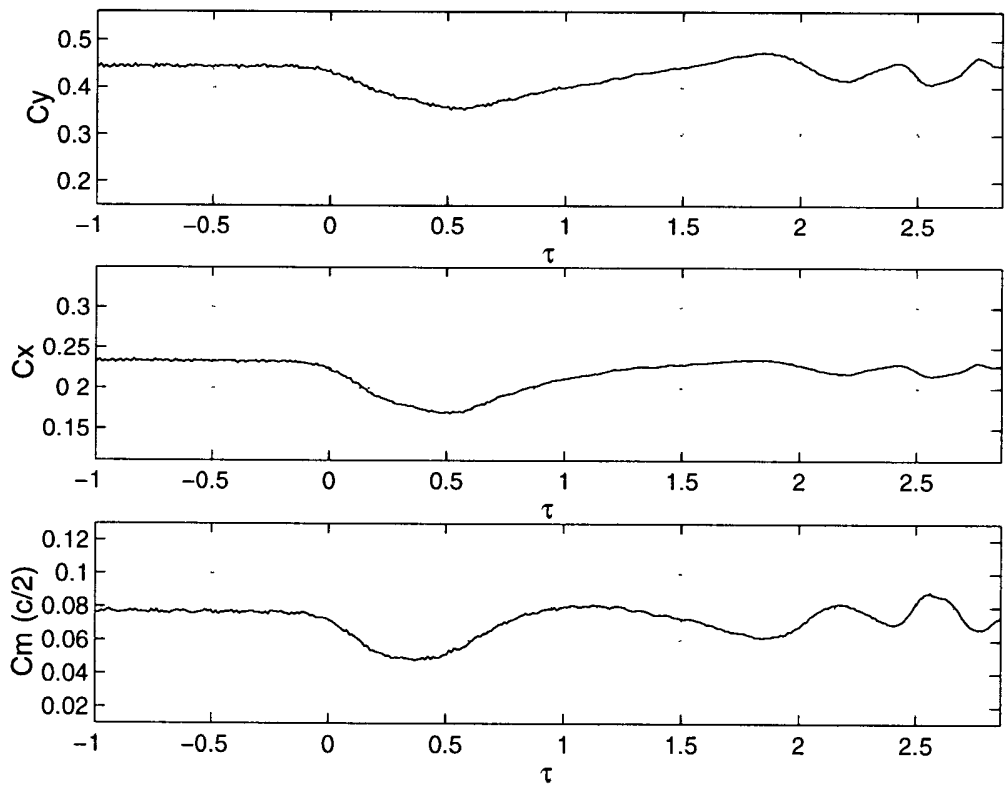

Figure B-34: Fluctuation in blade force and moment coefficients. $w / c=0.4, \rho_{2} / \rho_{1}=0.50$, $M_{\infty}=0.15$. 

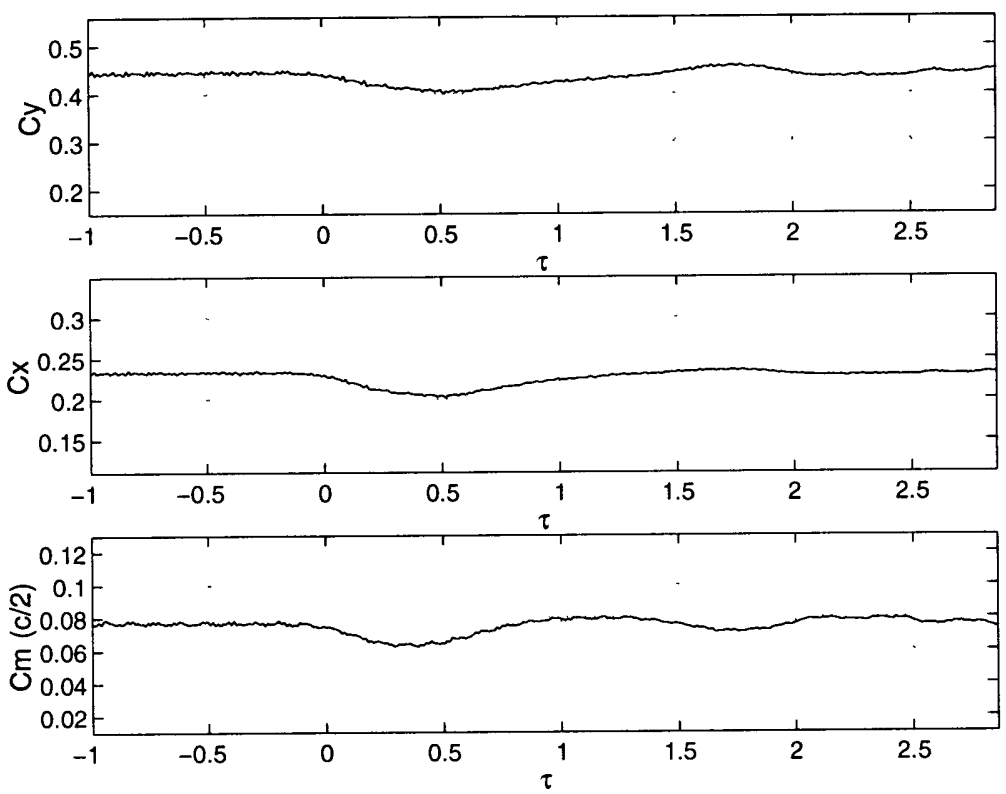

Figure B-35: Fluctuation in blade force and moment coefficients. $w / c=0.4, \rho_{2} / \rho_{1}=0.75$, $M_{\infty}=0.15$.
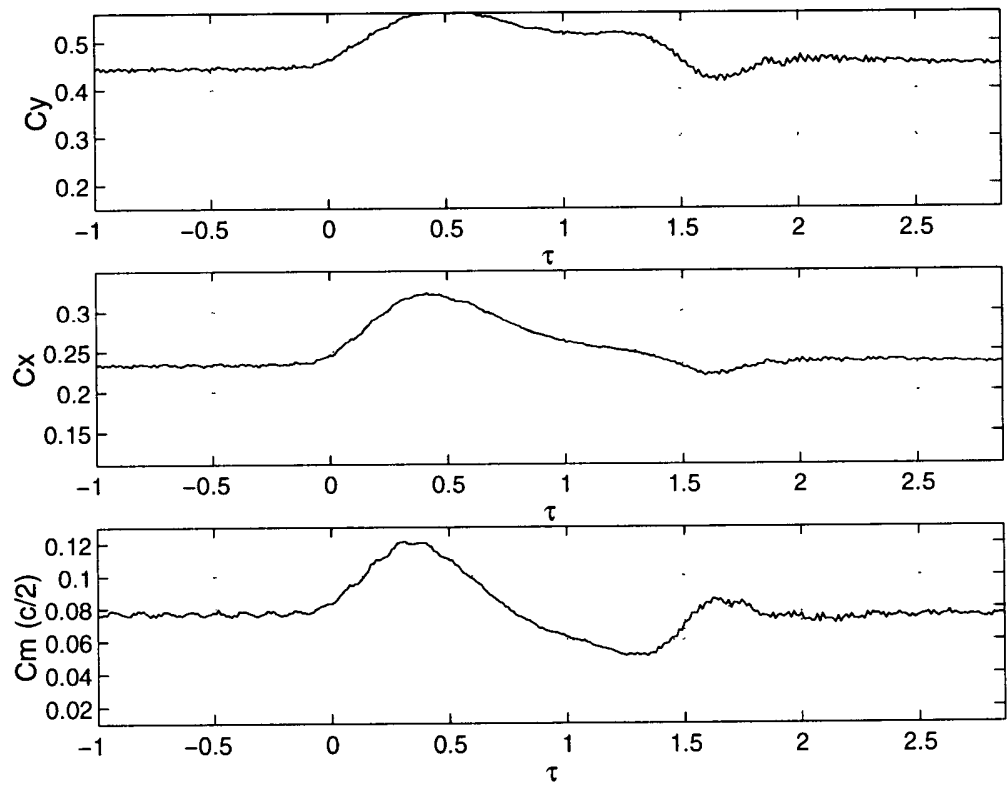

Figure B-36: Fluctuation in blade force and moment coefficients. $w / c=0.4, \rho_{2} / \rho_{1}=2.00$, $M_{\infty}=0.15$. 

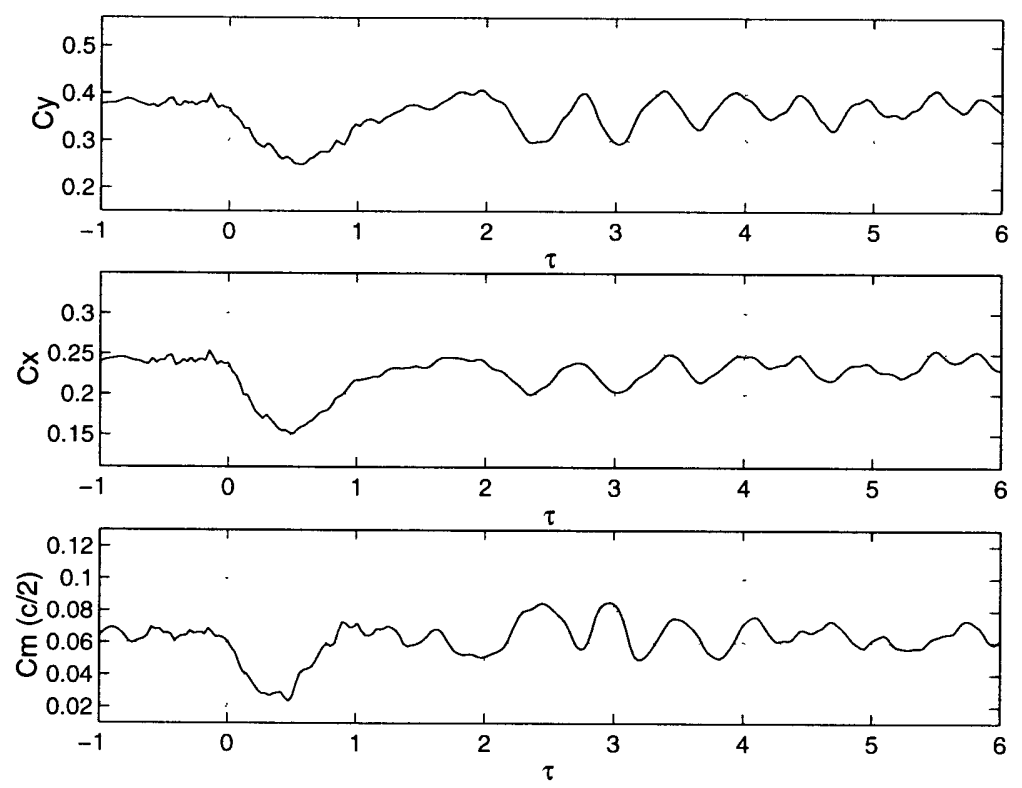

Figure B-37: Fluctuation in blade force and moment coefficients. $w / c=0.4, \rho_{2} / \rho_{1}=0.25$, $M_{\infty}=0.53$.
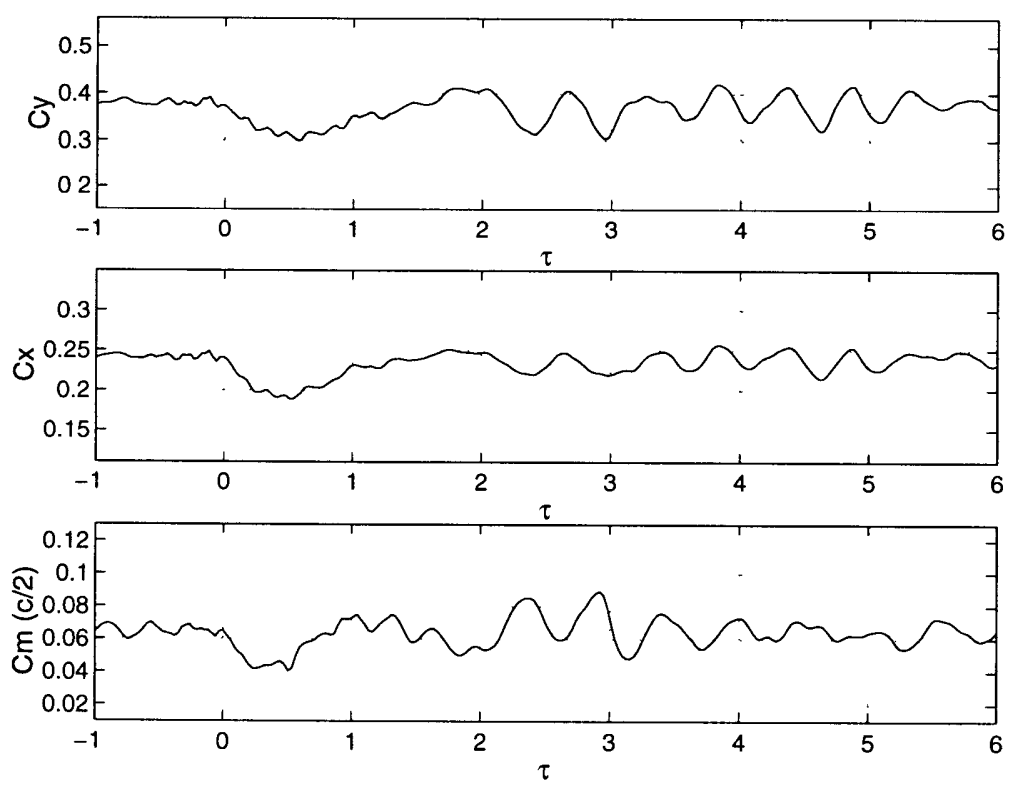

Figure B-38: Fluctuation in blade force and moment coefficients. $w / c=0.4, \rho_{2} / \rho_{1}=0.50$, $M_{\infty}=0.53$. 

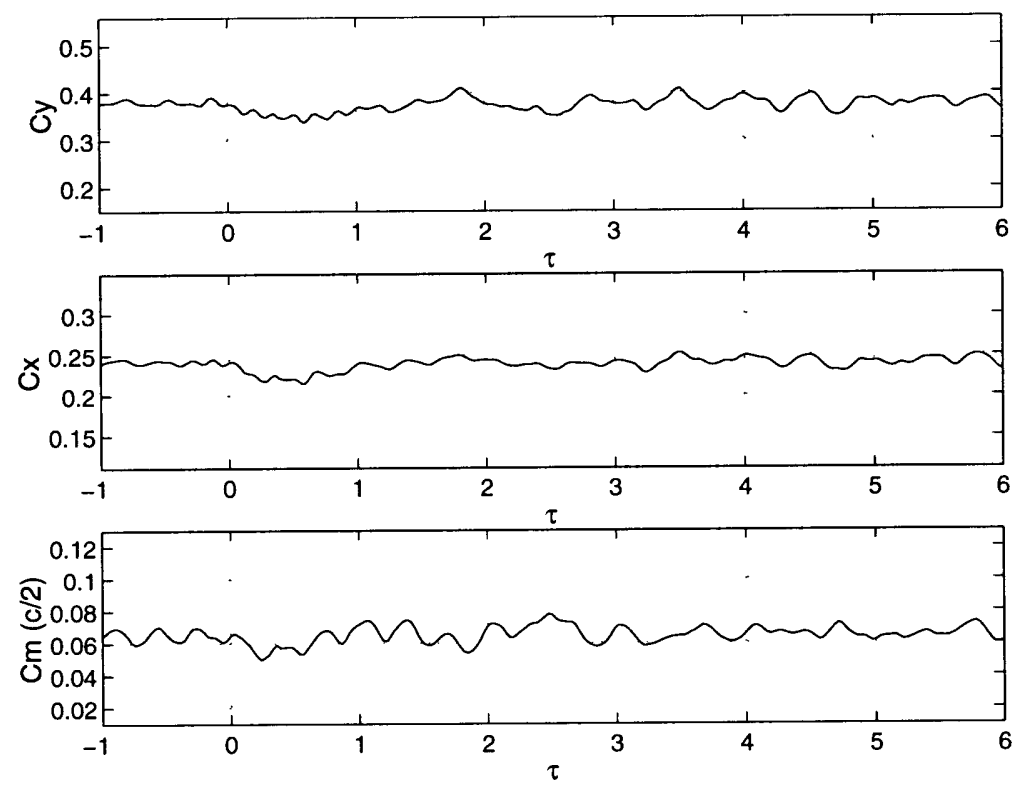

Figure B-39: Fluctuation in blade force and moment coefficients. $w / c=0.4, \rho_{2} / \rho_{1}=0.75$, $M_{\infty}=0.53$.
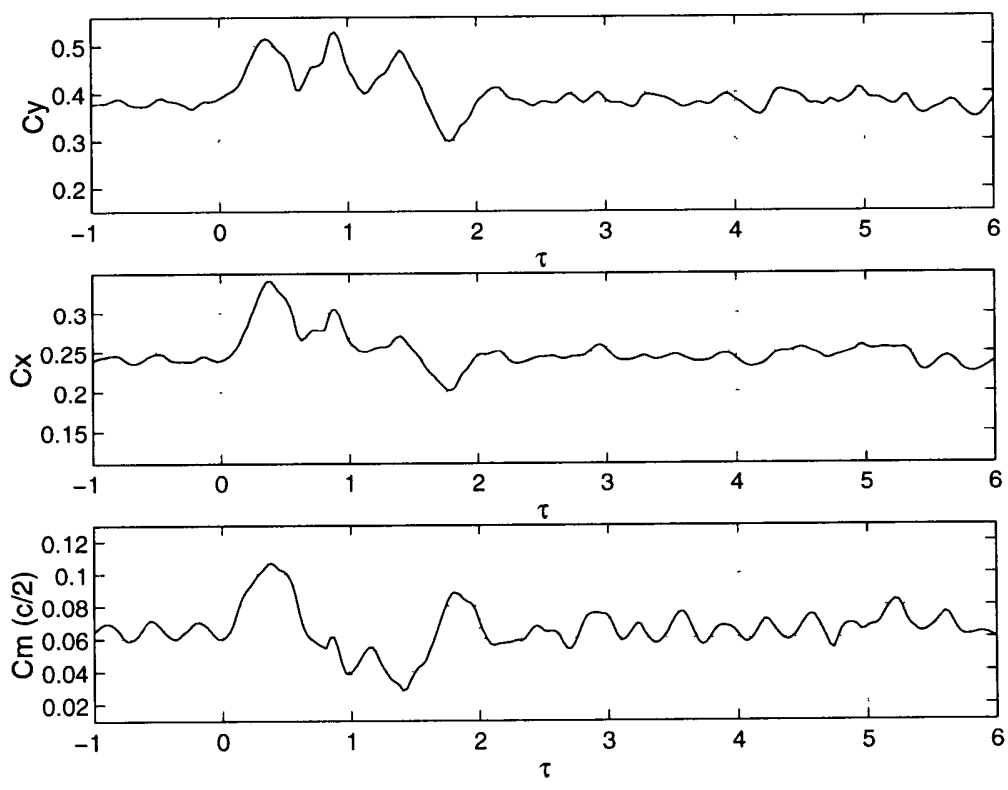

Figure B-40: Fluctuation in blade force and moment coefficients. $w / c=0.4, \rho_{2} / \rho_{1}=2.00$, $M_{\infty}=0.53$. 

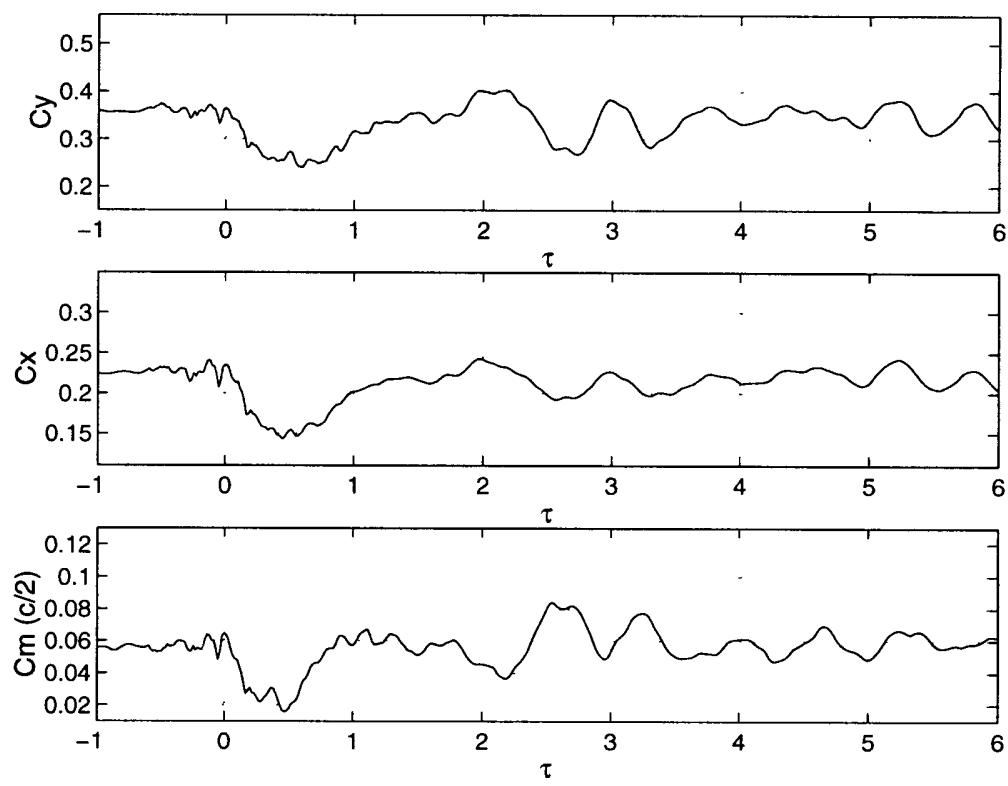

Figure B-41: Fluctuation in blade force and moment coefficients. $w / c=0.4, \rho_{2} / \rho_{1}=0.25$, $M_{\infty}=0.63$
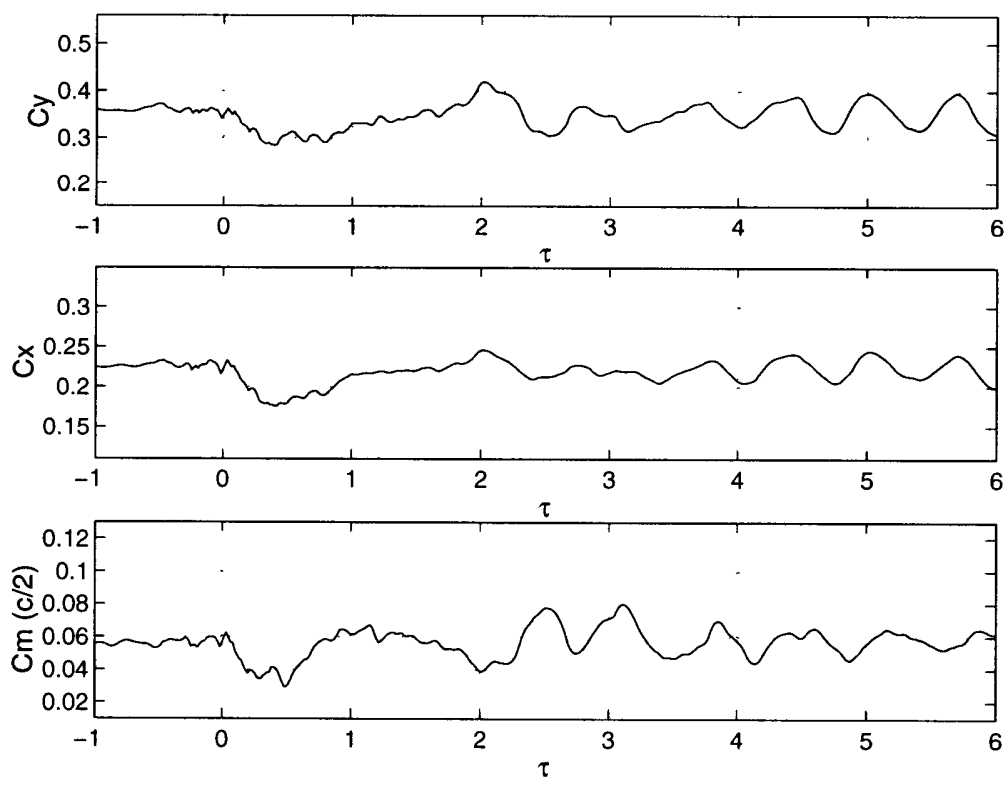

Figure B-42: Fluctuation in blade force and moment coefficients. $w / c=0.4, \rho_{2} / \rho_{1}=0.50$, $M_{\infty}=0.63$. 

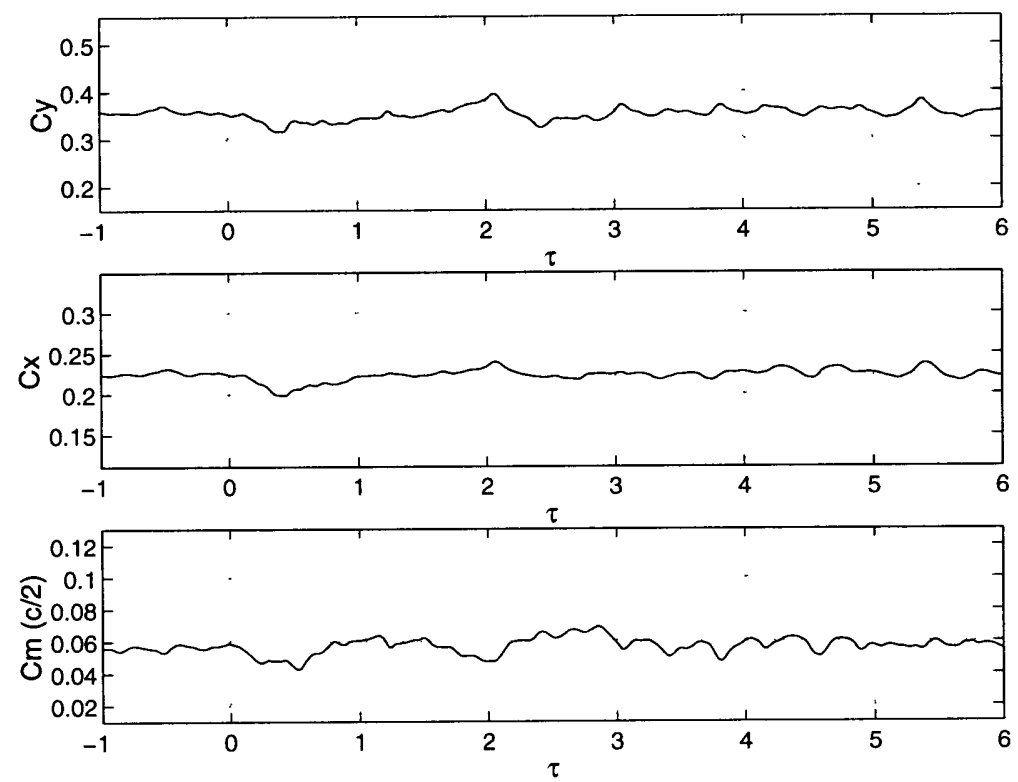

Figure B-43: Fluctuation in blade force and moment coefficients. $w / c=0.4, \rho_{2} / \rho_{1}=0.75$, $M_{\infty}=0.63$.
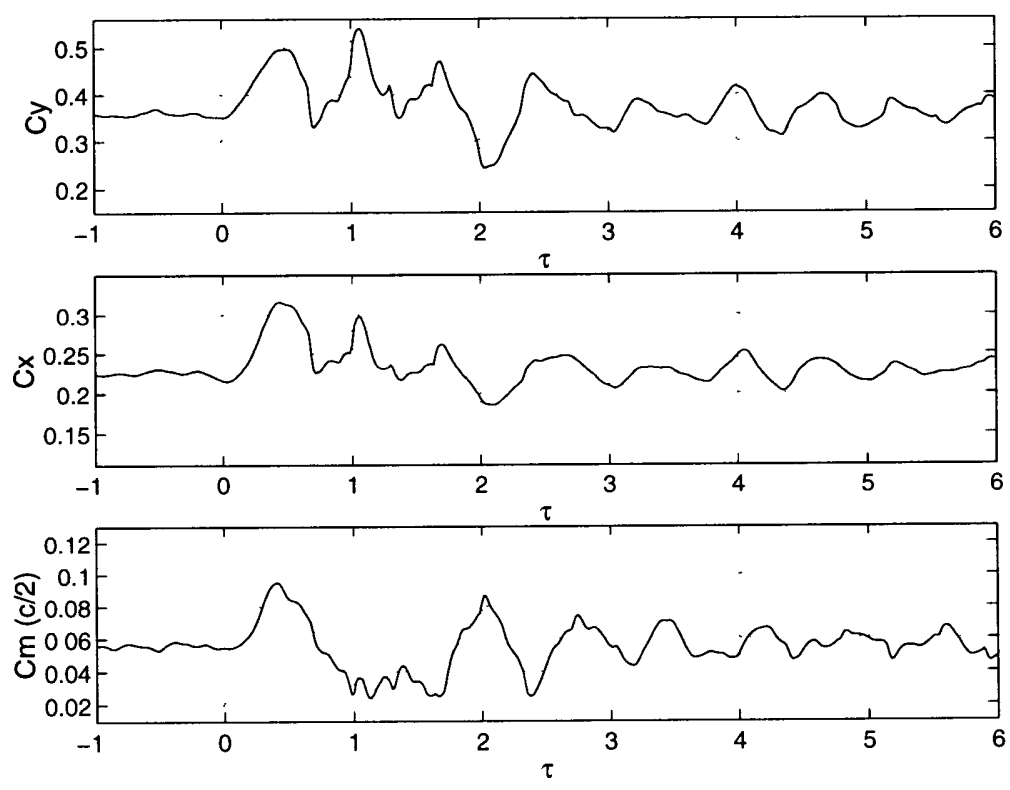

Figure B-44: Fluctuation in blade force and moment coefficients. $w / c=0.4, \rho_{2} / \rho_{1}=2.00$, $M_{\infty}=0.63$. 

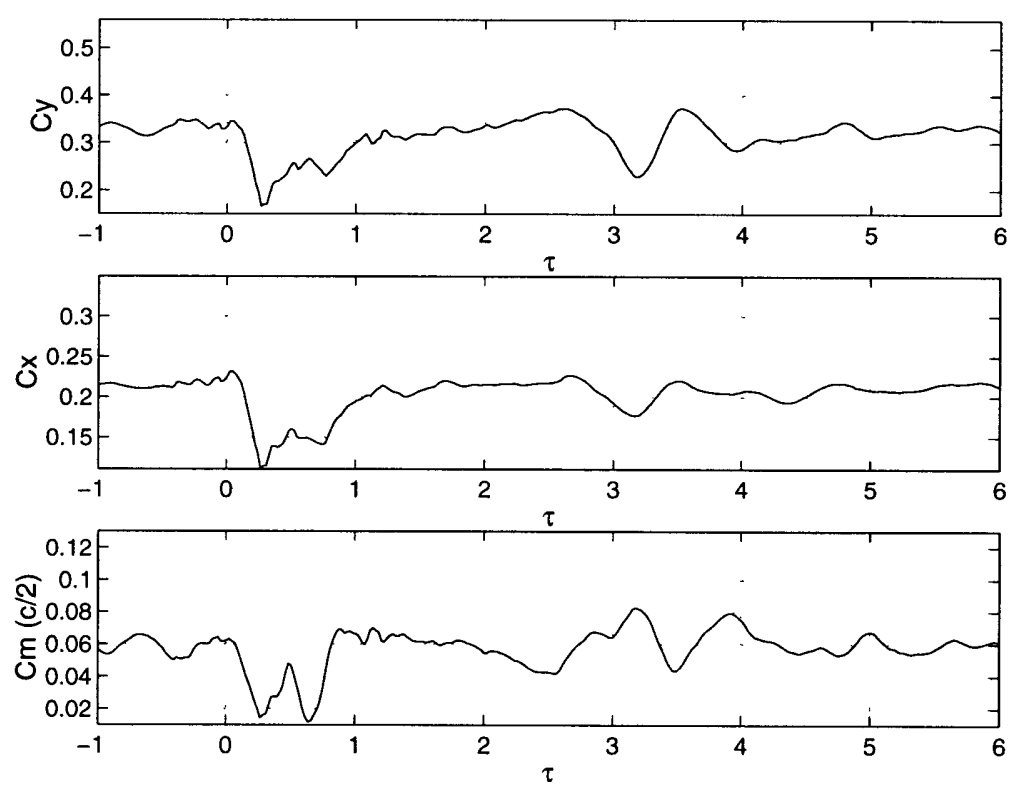

Figure B-45: Fluctuation in blade force and moment coefficients. $w / c=0.4, \rho_{2} / \rho_{1}=0.25$, $M_{\infty}=0.87$.
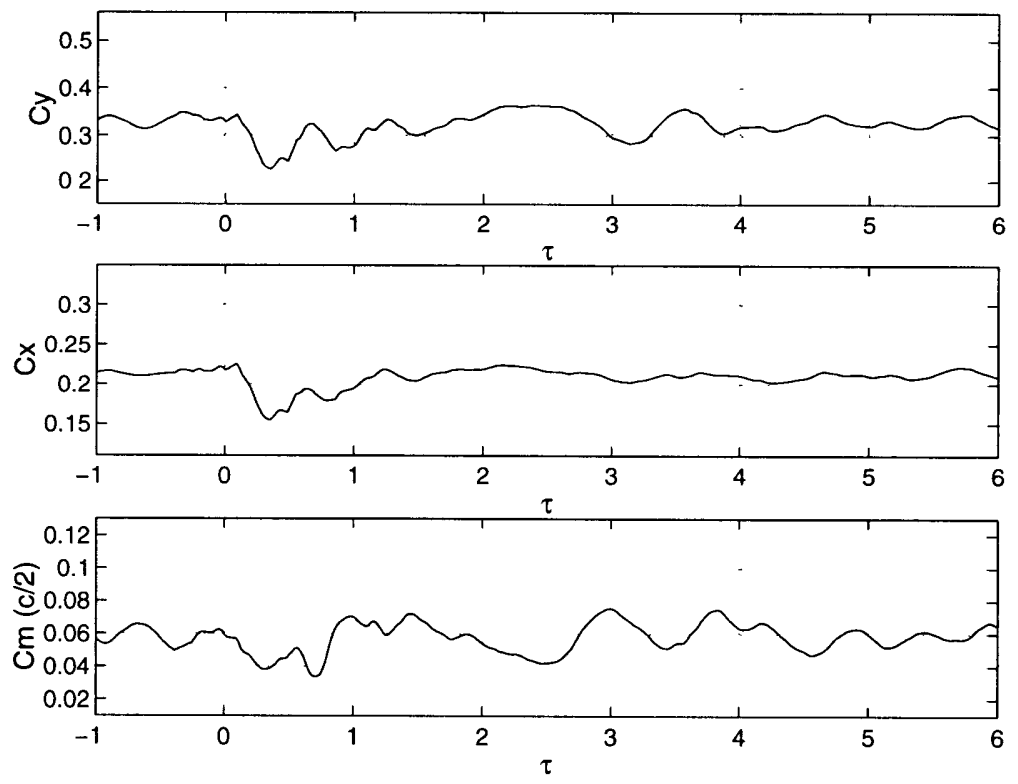

Figure B-46: Fluctuation in blade force and moment coefficients. $w / c=0.4, \rho_{2} / \rho_{1}=0.50$, $M_{\infty}=0.87$. 

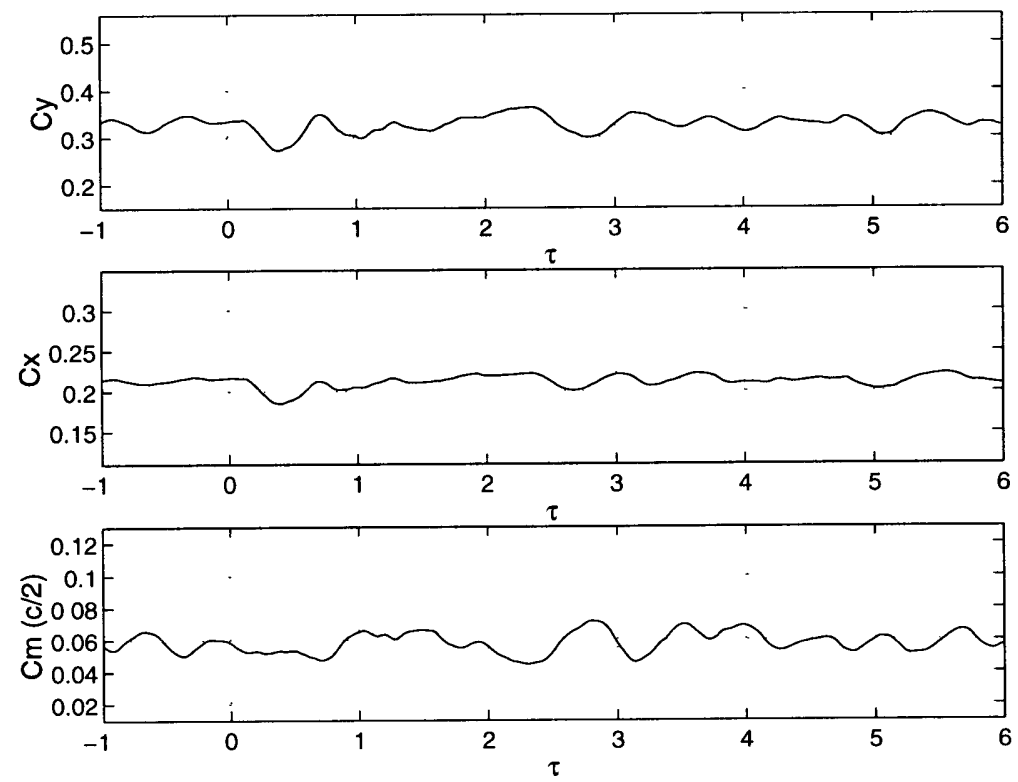

Figure B-47: Fluctuation in blade force and moment coefficients. $w / c=0.4, \rho_{2} / \rho_{1}=0.75$, $M_{\infty}=0.87$.
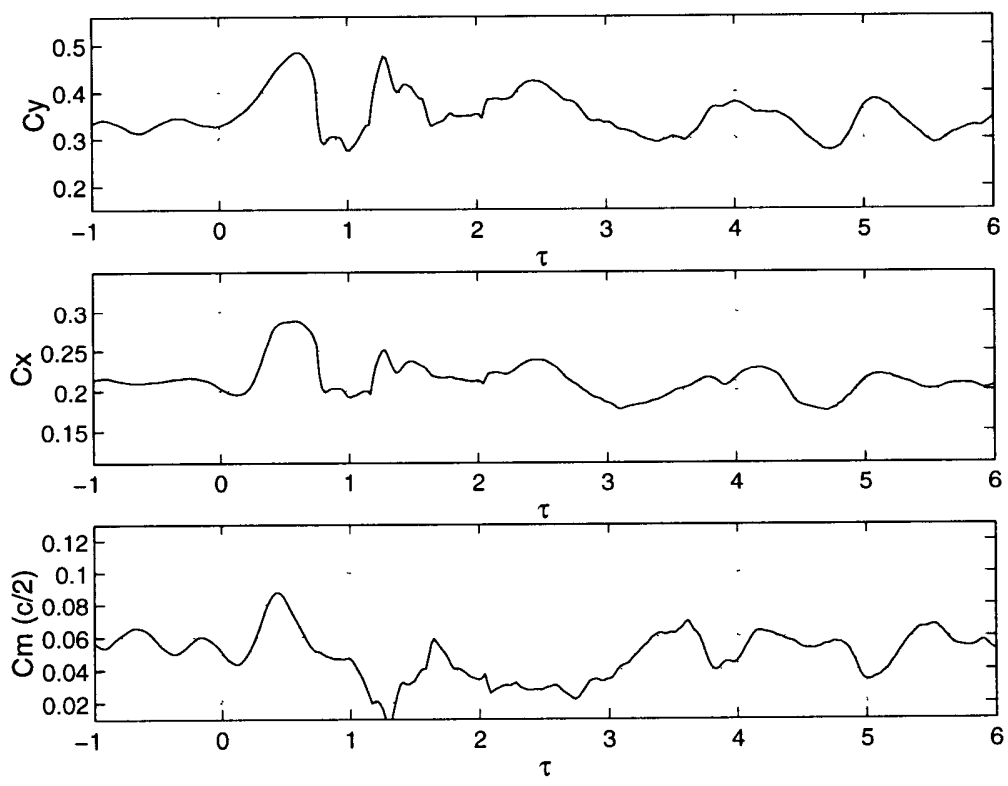

Figure B-48: Fluctuation in blade force and moment coefficients. $w / c=0.4, \rho_{2} / \rho_{1}=2.00$, $M_{\infty}=0.87$. 

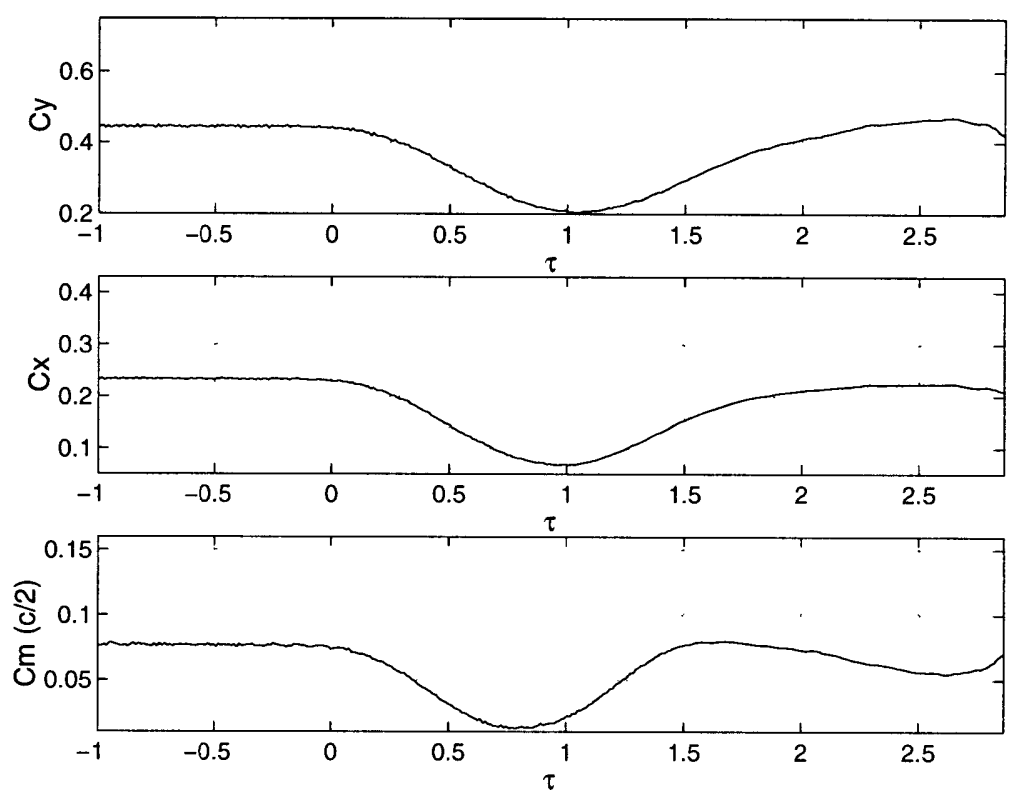

Figure B-49: Fluctuation in blade force and moment coefficients. $w / c=1.0, \rho_{2} / \rho_{1}=0.25$, $M_{\infty}=0.15$.
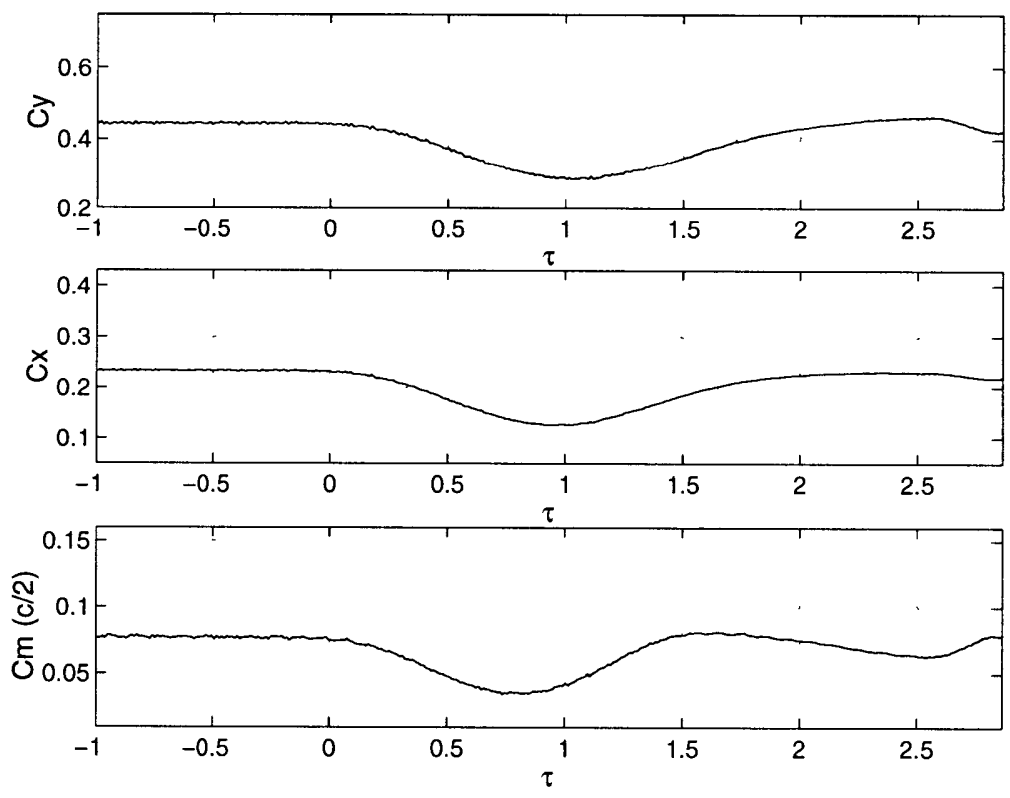

Figure B-50: Fluctuation in blade force and moment coefficients. $w / c=1.0, \rho_{2} / \rho_{1}=0.50$, $M_{\infty}=0.15$. 

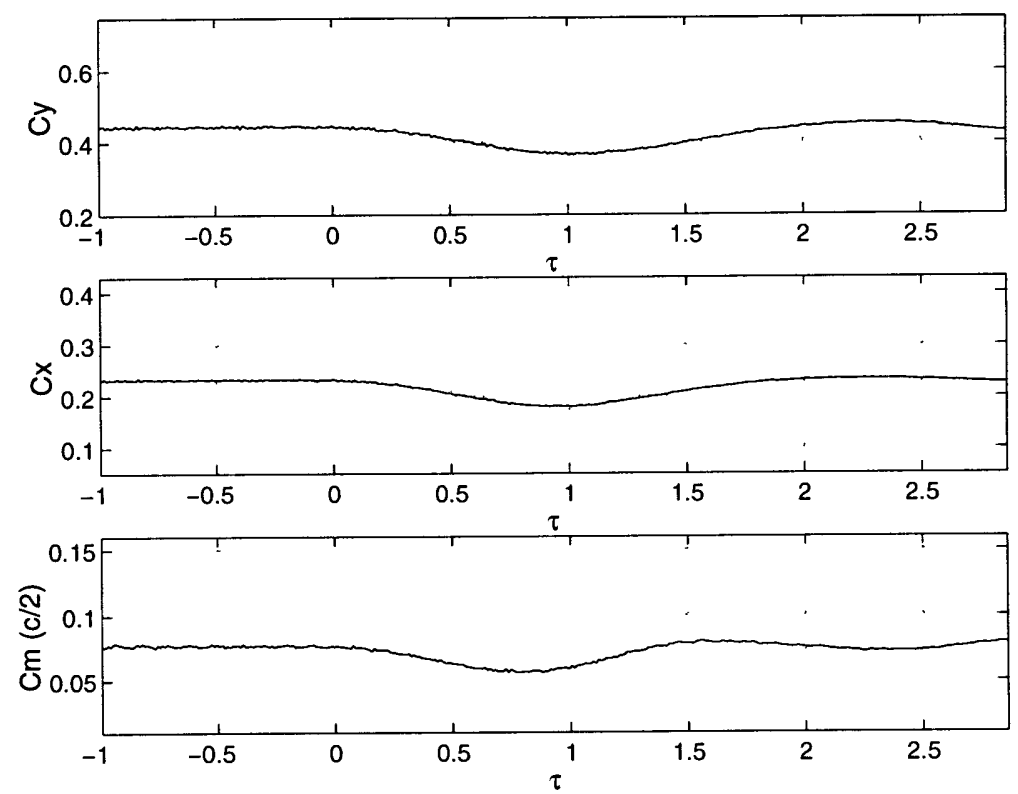

Figure B-51: Fluctuation in blade force and moment coefficients. $w / c=1.0, \rho_{2} / \rho_{1}=0.75$, $M_{\infty}=0.15$.
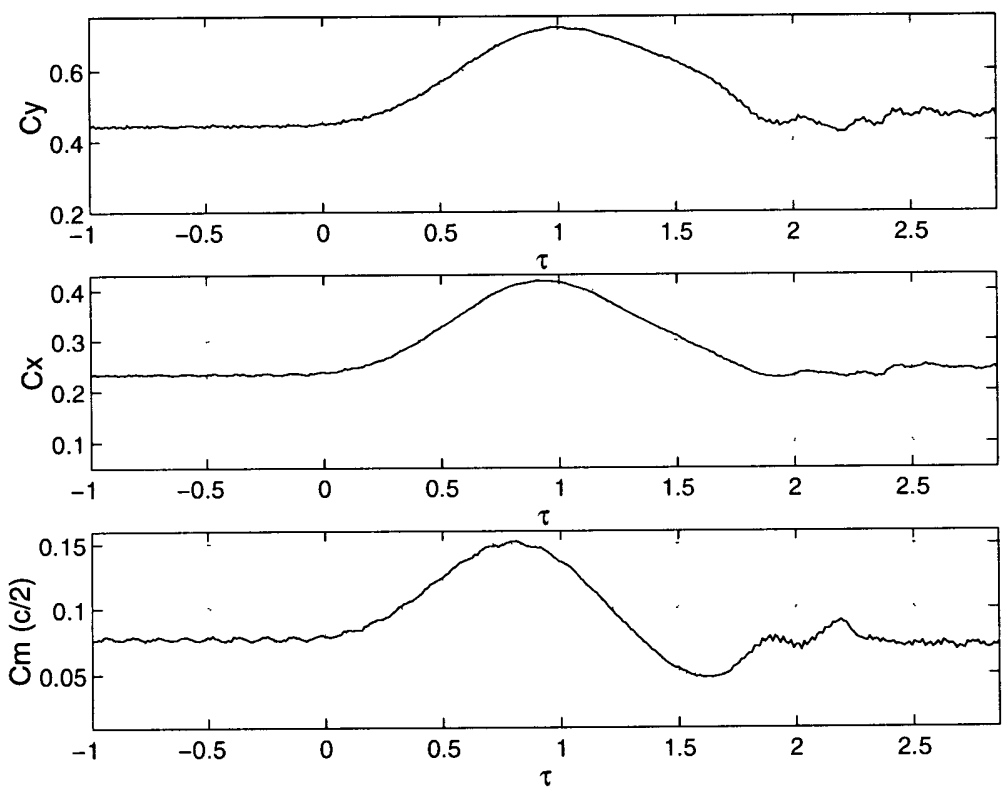

Figure B-52: Fluctuation in blade force and moment coefficients. $w / c=1.0, \rho_{2} / \rho_{1}=2.00$, $M_{\infty}=0.15$. 


\section{ApPENDix C}

\section{Compressibility Scaling Of The Maximum ForCe AND MOMENT FLUCTUATIONS}

This Appendix includes plots for the maximum fluctuations in the (1) azimuthal force coefficient $C y,(2)$ axial force coefficient $C x$ and (3) moment coefficient $C m$ for the primary response.

Each force and moment coefficient is scaled by the Prandtl-Glauert compressibility factor $\sqrt{1-M_{\infty}^{2}}$. The results for the $M_{\infty}=0.15$ calculation are overlayed in each plot for comparison purposes.

Note the maximum axial force coefficient fluctuation for the inviscid flow is not available. 


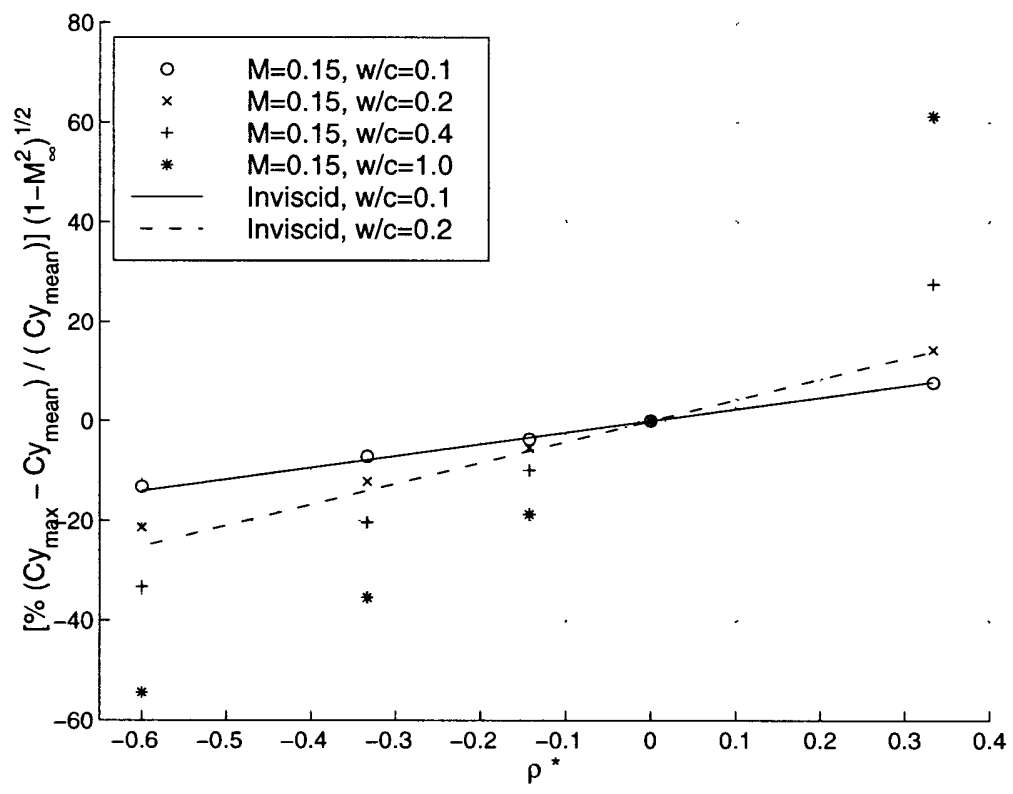

Figure C-1: Comparison of the $M_{\infty}=0.15$ viscous results and the inviscid results for the maximum fluctuation in the azimuthal force coefficient.

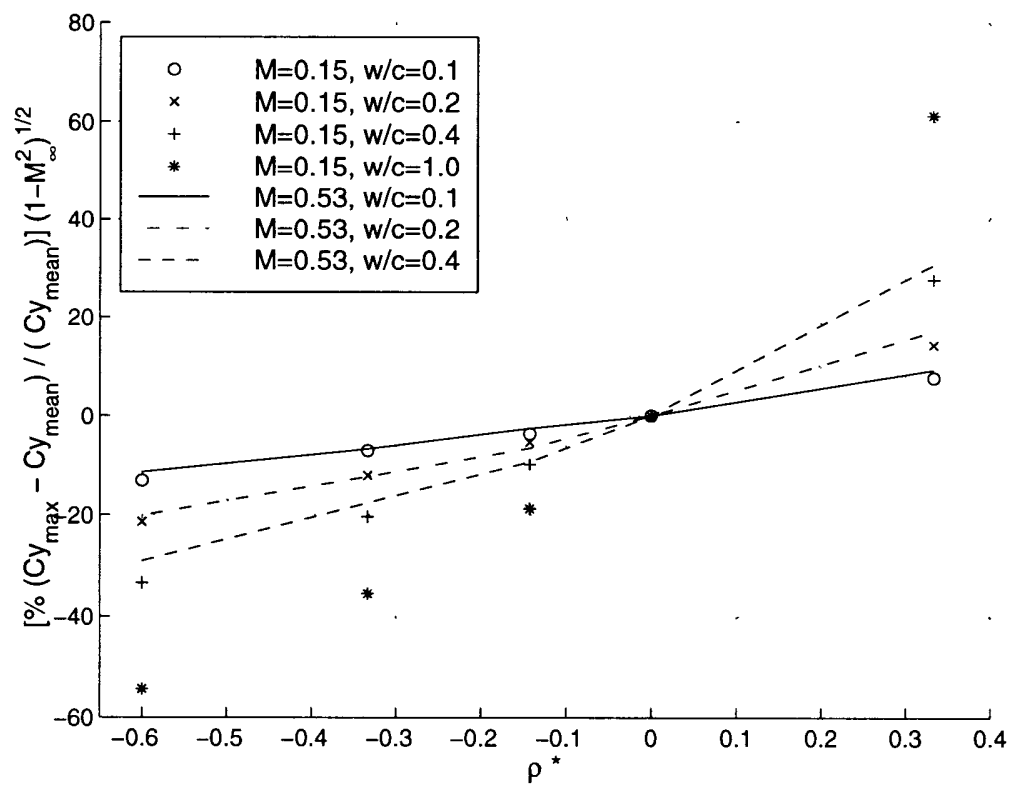

Figure C-2: Comparison of the $M_{\infty}=0.15$ and the $M_{\infty}=0.53$ viscous results for the maximum fluctuation in the azimuthal force coefficient. 


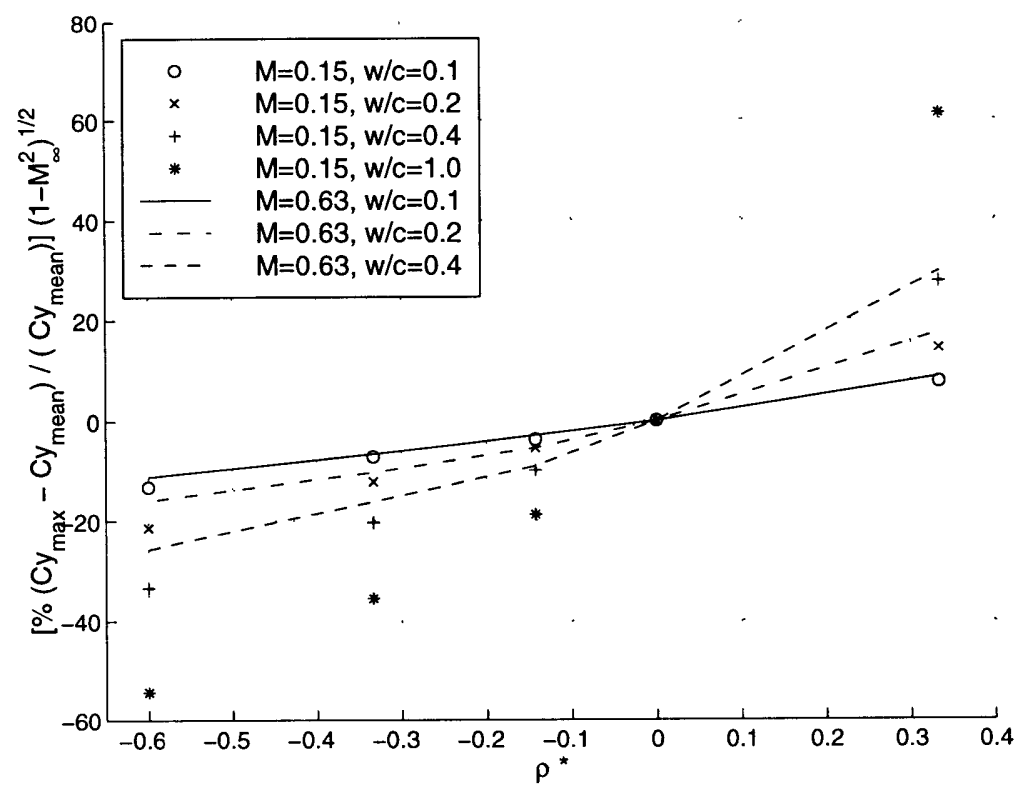

Figure C-3: Comparison of the $M_{\infty}=0.15$ and the $M_{\infty}=0.63$ viscous results for the maximum fluctuation in the azimuthal force coefficient.

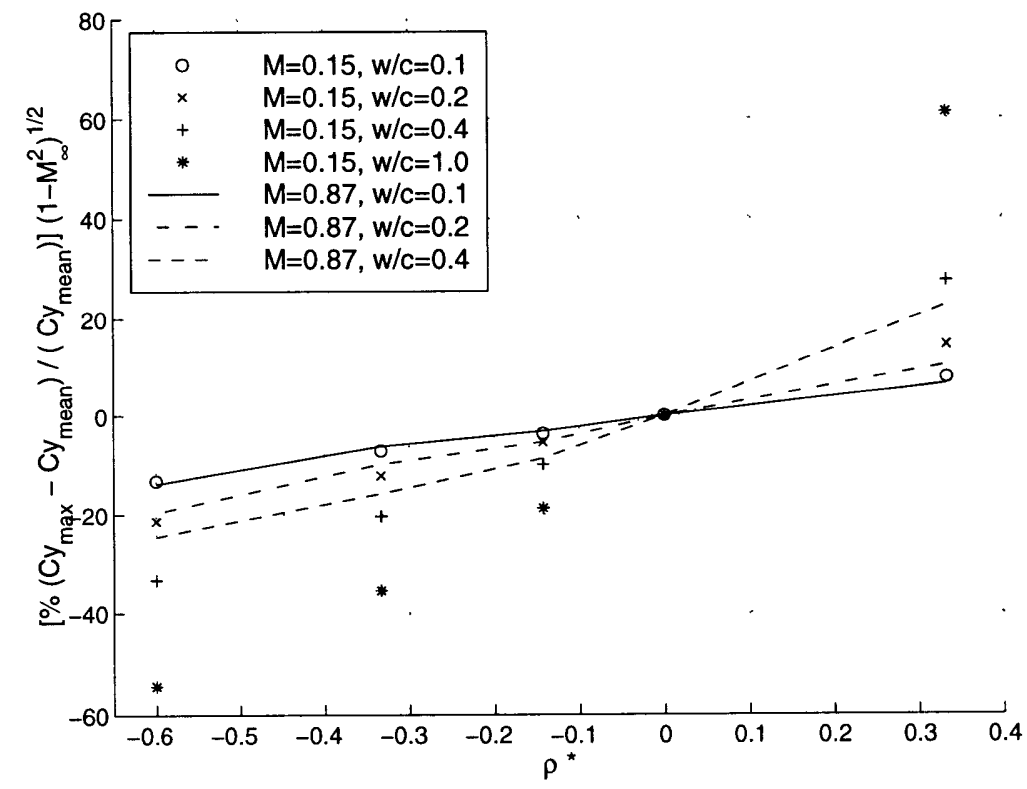

Figure C-4: Comparison of the $M_{\infty}=0.15$ and the $M_{\infty}=0.87$ viscous results for the maximum fluctuation in the azimuthal force coefficient. 


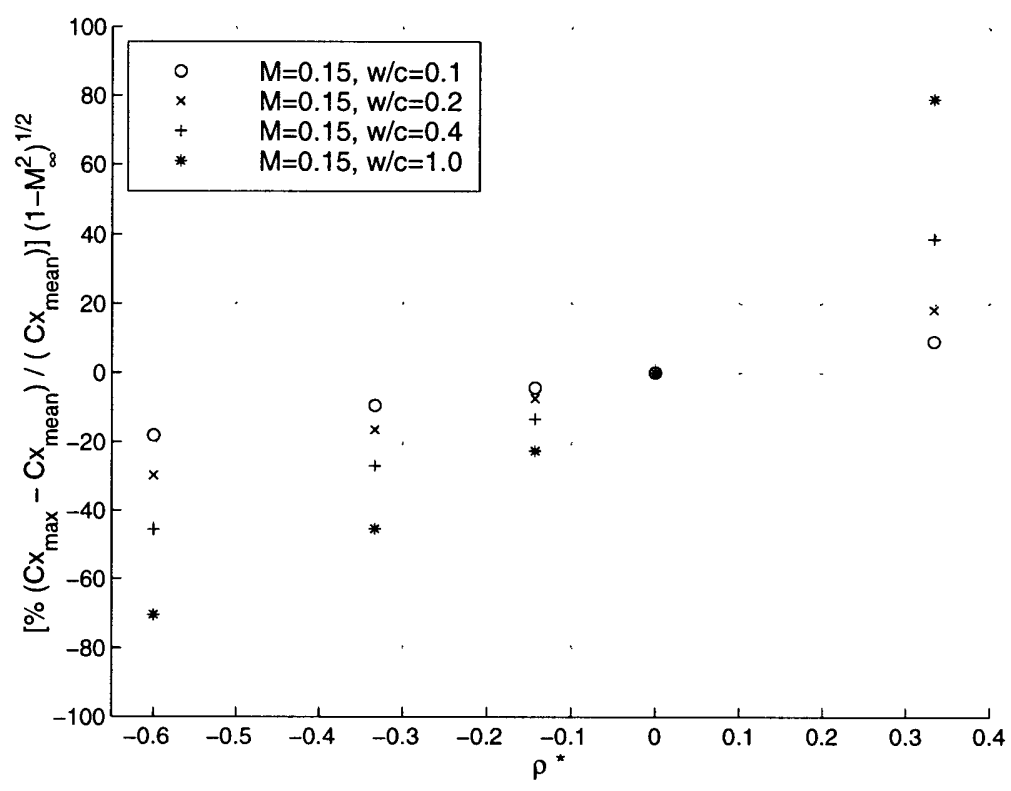

Figure C-5: $M_{\infty}=0.15$ viscous result for the maximum fluctuation in the axial force coefficient.

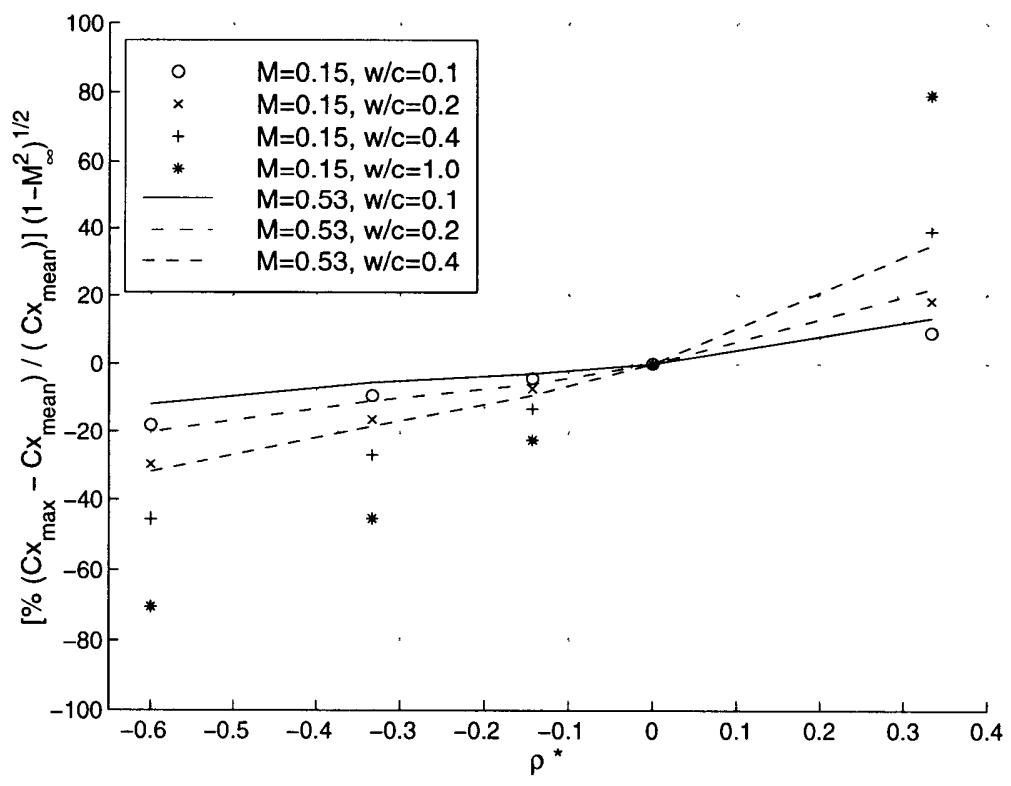

Figure C-6: Comparison of the $M_{\infty}=0.15$ and the $M_{\infty}=0.53$ viscous results for the maximum fluctuation in the axial force coefficient. 


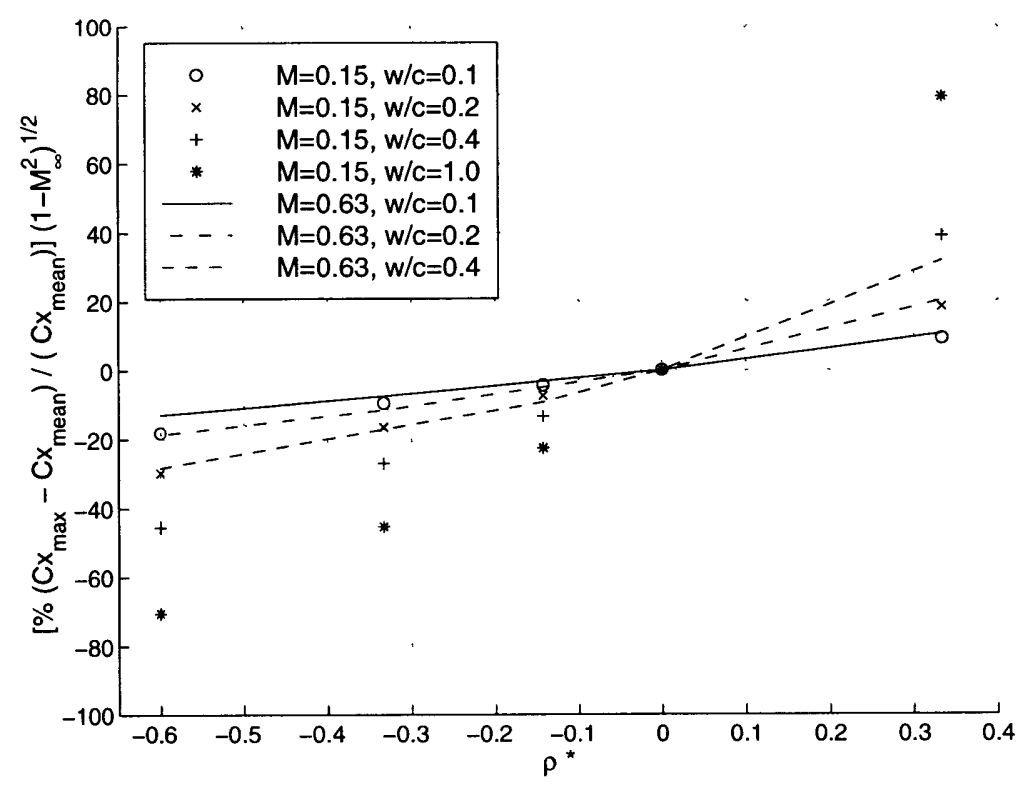

Figure C-7: Comparison of the $M_{\infty}=0.15$ and the $M_{\infty}=0.63$ viscous results for the maximum fluctuation in the axial force coefficient.

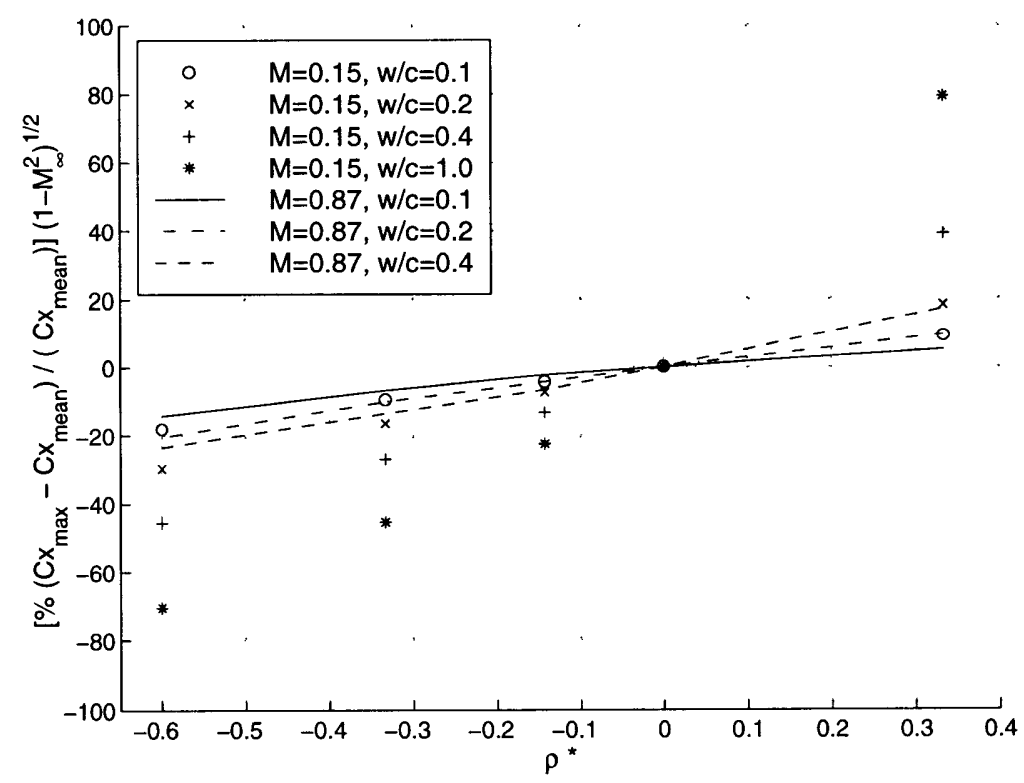

Figure C-8: Comparison of the $M_{\infty}=0.15$ and the $M_{\infty}=0.87$ viscous results for the maximum fluctuation in the axial force coefficient. 


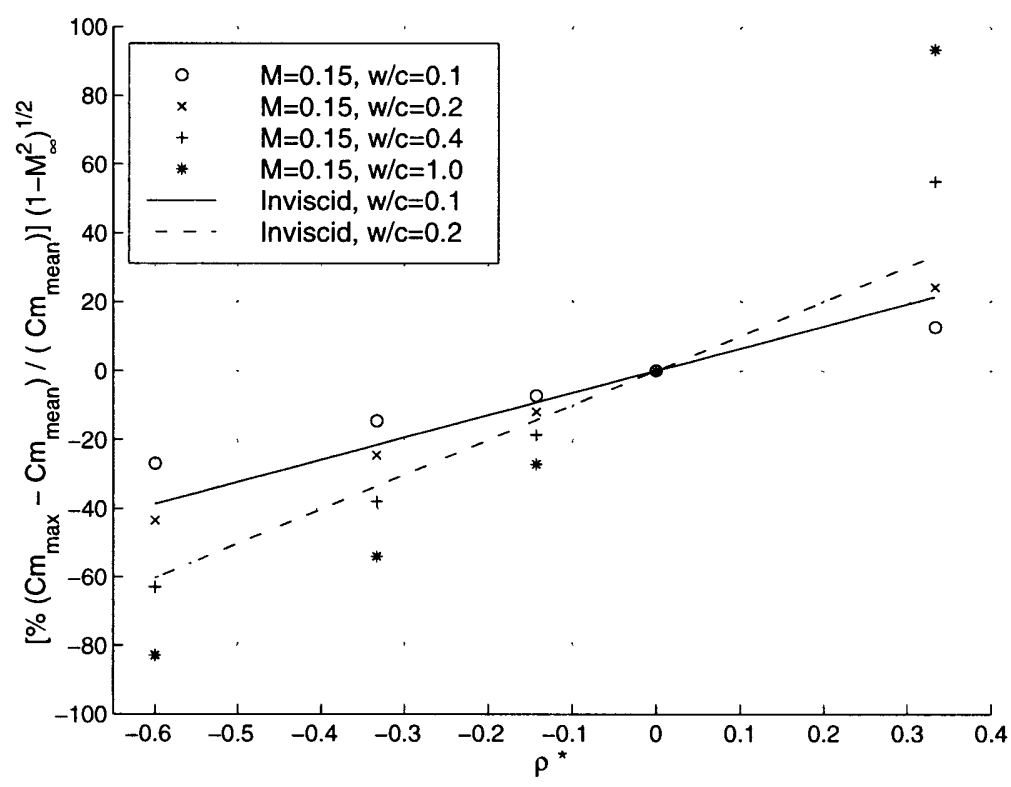

Figure C-9: Comparison of the $M_{\infty}=0.15$ viscous results and the inviscid results for the maximum fluctuation in the moment coefficient.

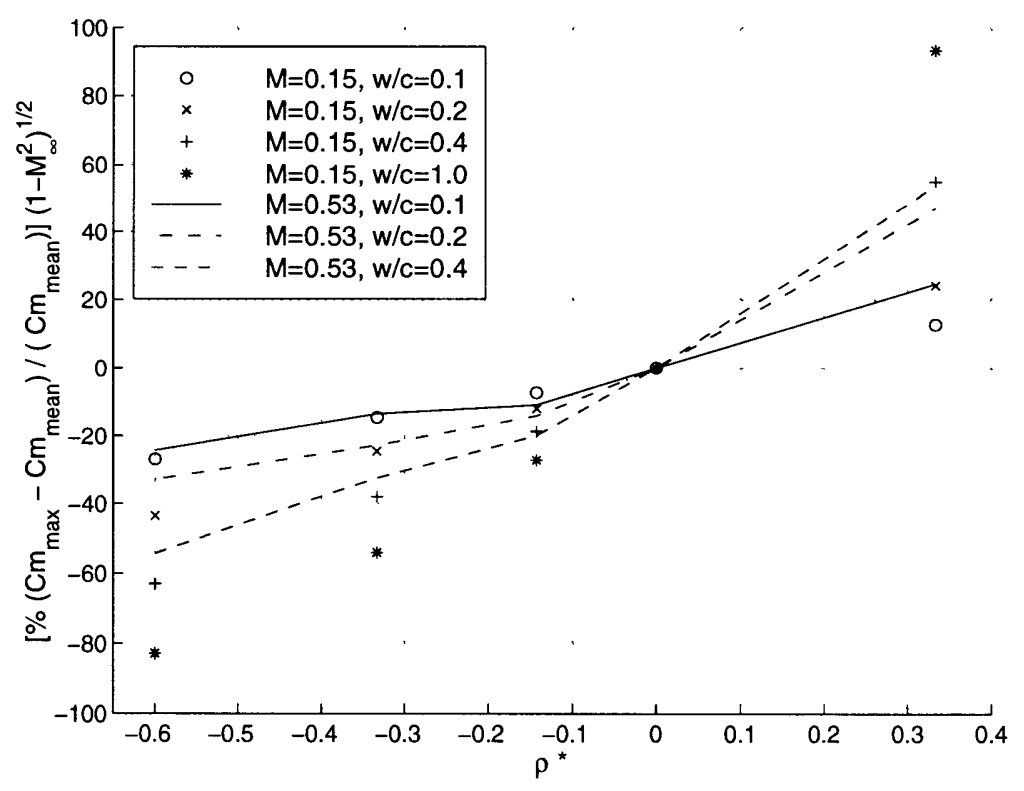

Figure C-10: Comparison of the $M_{\infty}=0.15$ and the $M_{\infty}=0.53$ viscous results for the maximum fluctuation in the moment coefficient. 


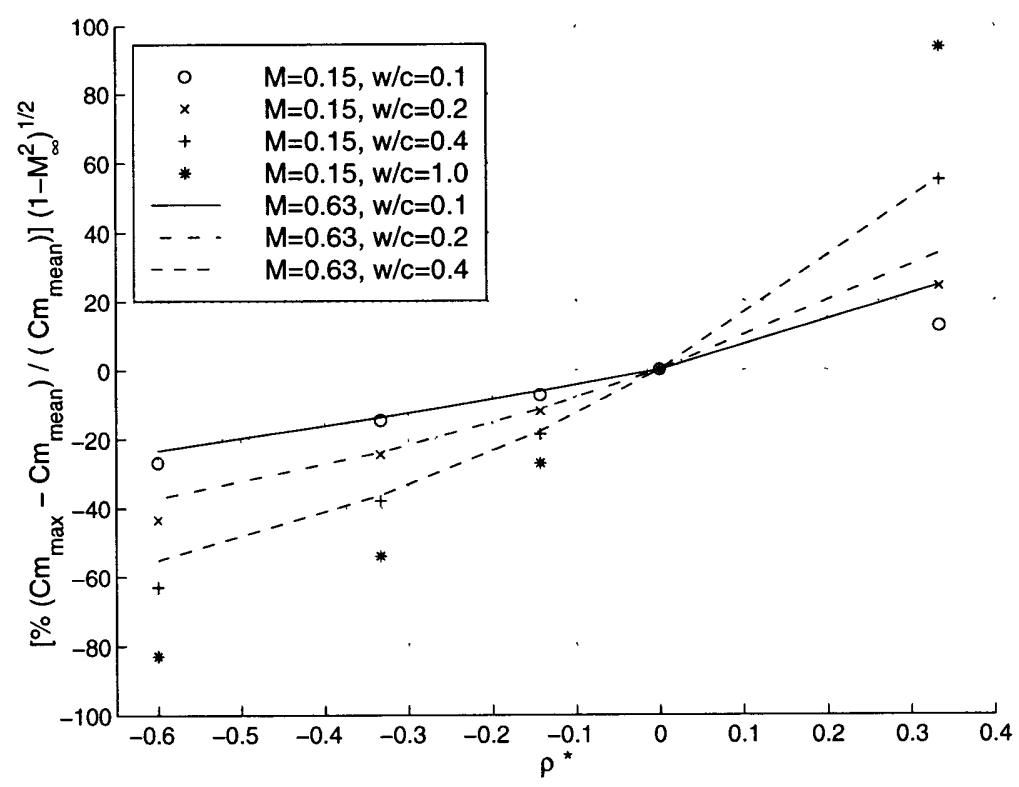

Figure C-11: Comparison of the $M_{\infty}=0.15$ and the $M_{\infty}=0.63$ viscous results for the maximum fluctuation in the moment coefficient.

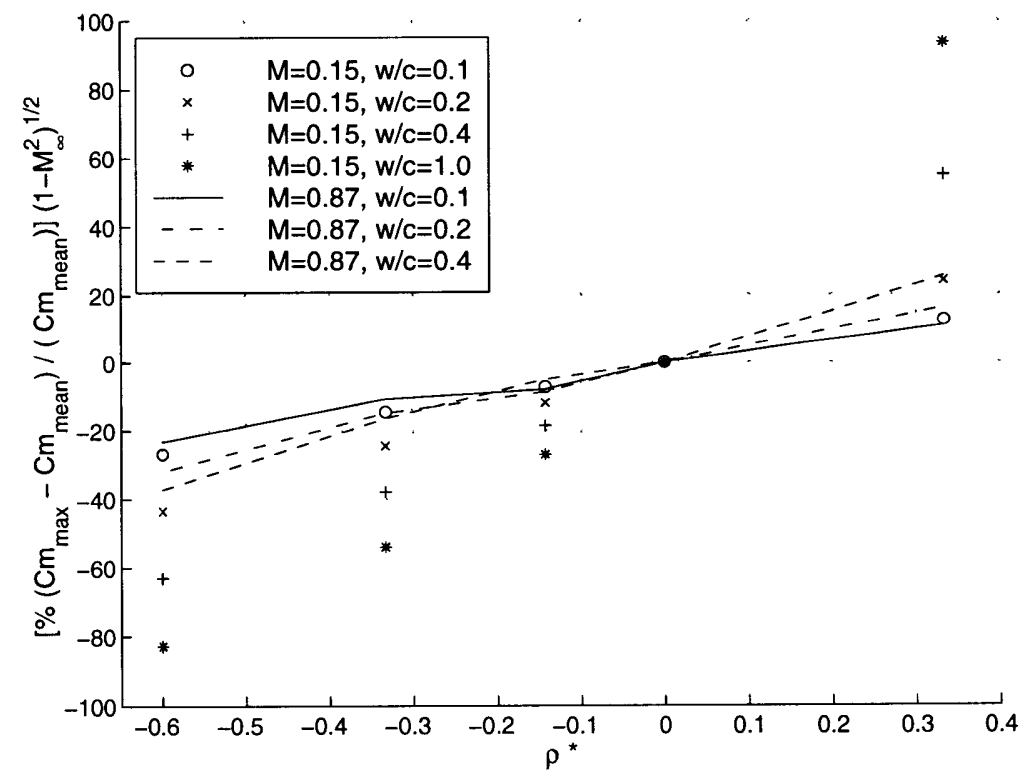

Figure C-12: Comparison of the $M_{\infty}=0.15$ and the $M_{\infty}=0.87$ viscous results for the maximum fluctuation in the moment coefficient. 


\section{APPENDIX D}

\section{Cascade Model : Matlab Source CODE}

Filename: cascade.m

$\%$ [Cascade flow model v7.0]

$\%$ Written by Sanith Wijesinghe

\% MIT Gas Turbine Lab. July 1998.

$\%$

\% Matlab script file.

$\%$

$\%$ Lift and moment fluctuation on a flat plate cascade induced by a

$\%$ convecting density wake.

$\%$

$\%$ Notes :

$\%$ o Cascade flat plate extends from -0.5 to +0.5 .

$\%$ o Uncomment lines where indicated for either steady or quasi-steady

$\%$ simulation.

$\%$ List of Symbols:

$\% \mathrm{~N} \quad=$ no. of vortex panels 


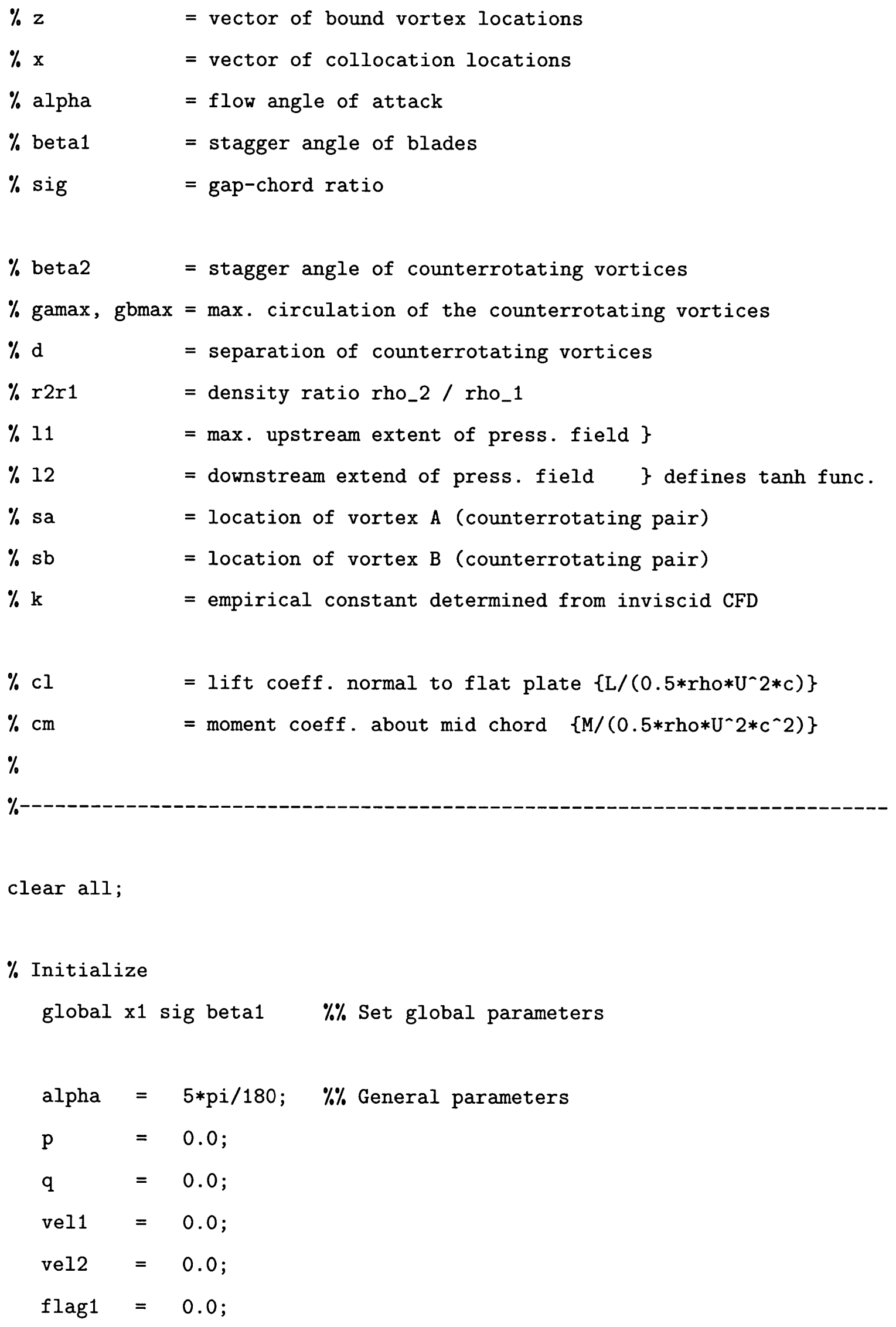




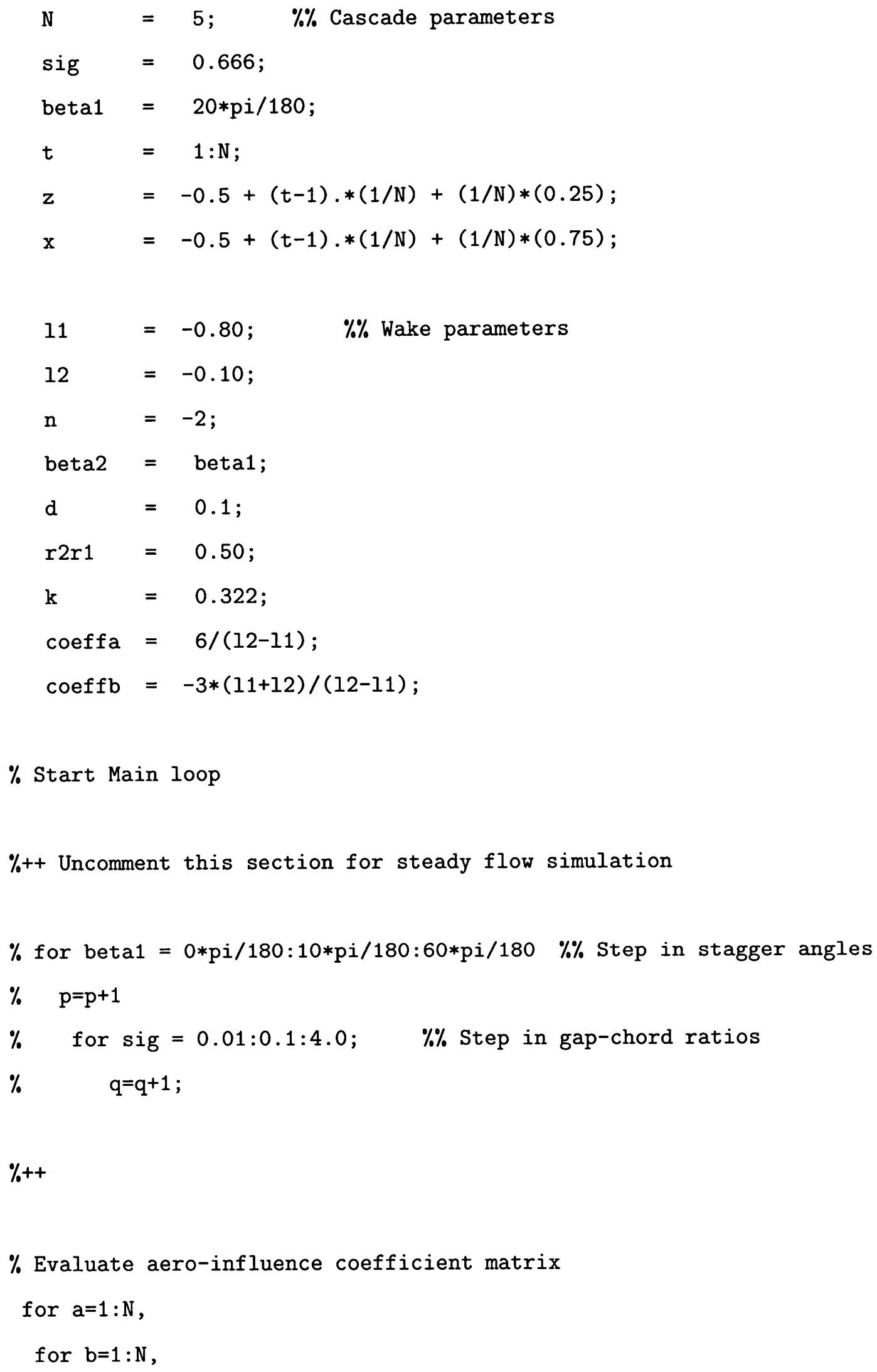




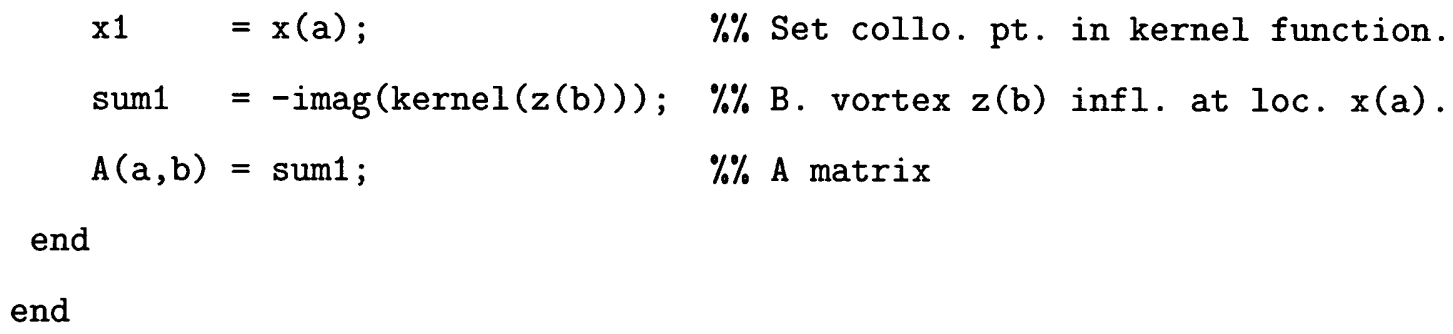

$\%$ Hyperbolic tangent variation in vortex strength

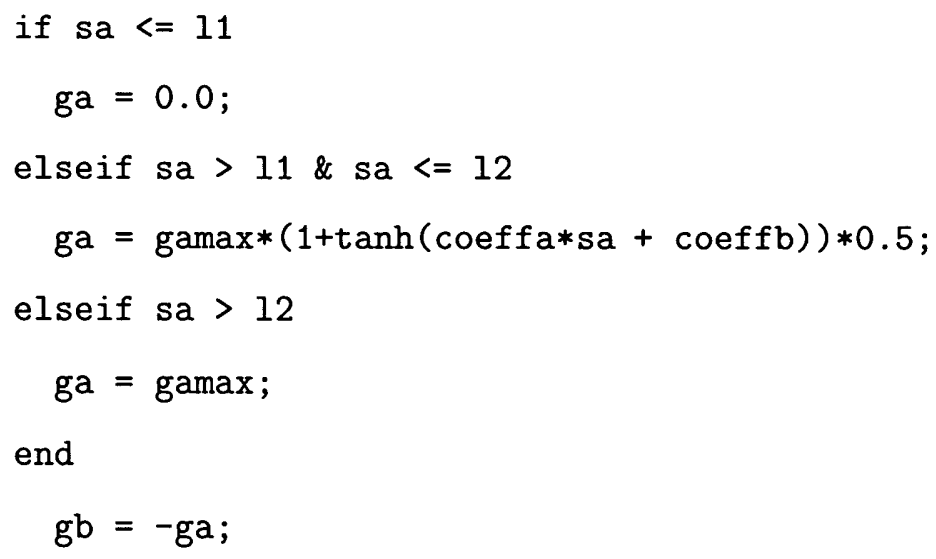


for $v=1: N \%$ Counterrotating Vortex pair infl. at panels + 1.e.

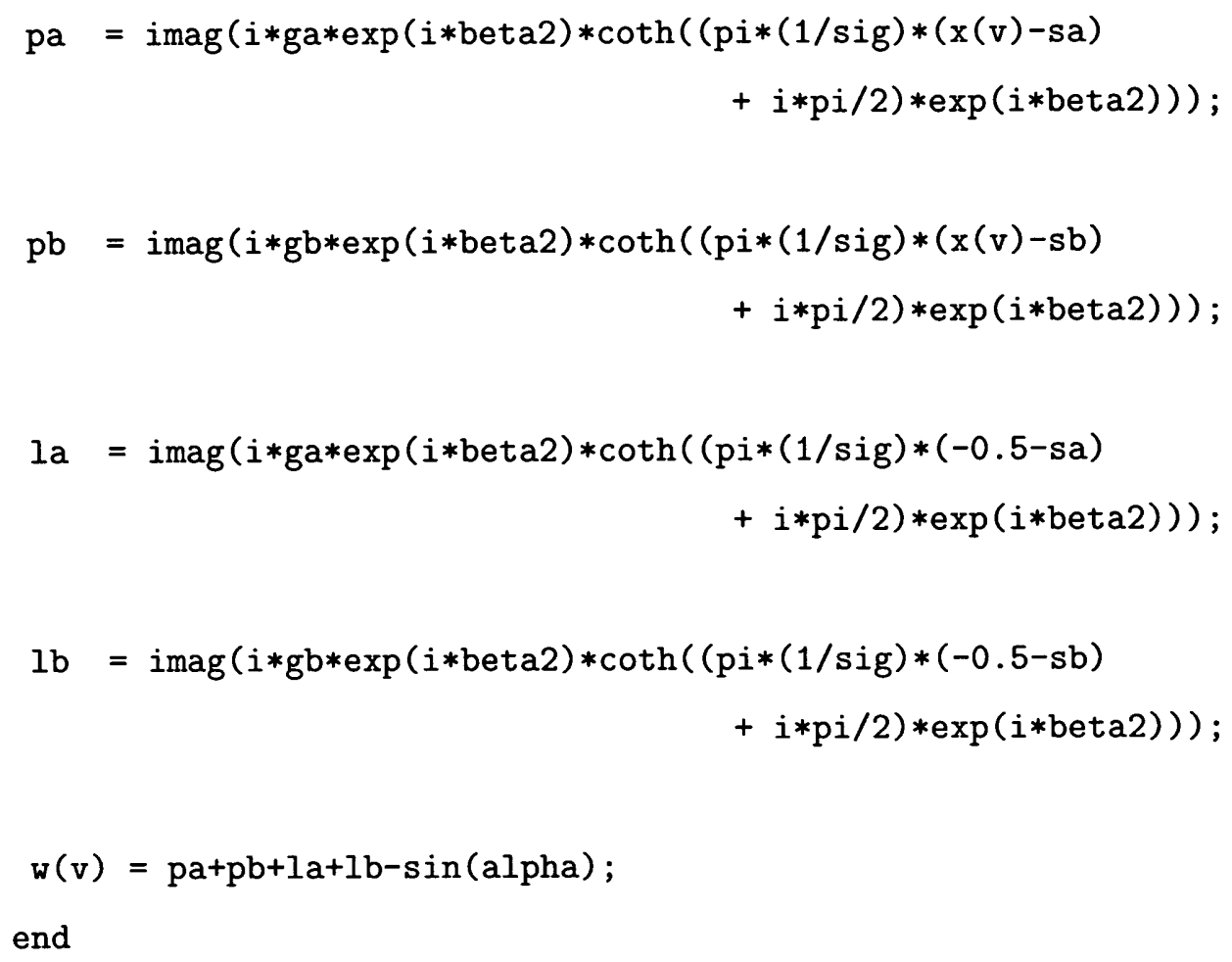

$\%$ Solution vector

$$
\begin{aligned}
& \operatorname{out1}(p, q)=n ; \\
& \operatorname{out2}(p, q)=c 1 ; \\
& \operatorname{out} 3(p, q)=c m ; \\
& \operatorname{out} 4(p, q)=\operatorname{kint} ; \\
& \operatorname{out} 5(p, q)=\operatorname{sig} ; \\
& \operatorname{out} 6(p, q)=\operatorname{beta1} ;
\end{aligned}
$$

\% Calculate gamax and gbmax 


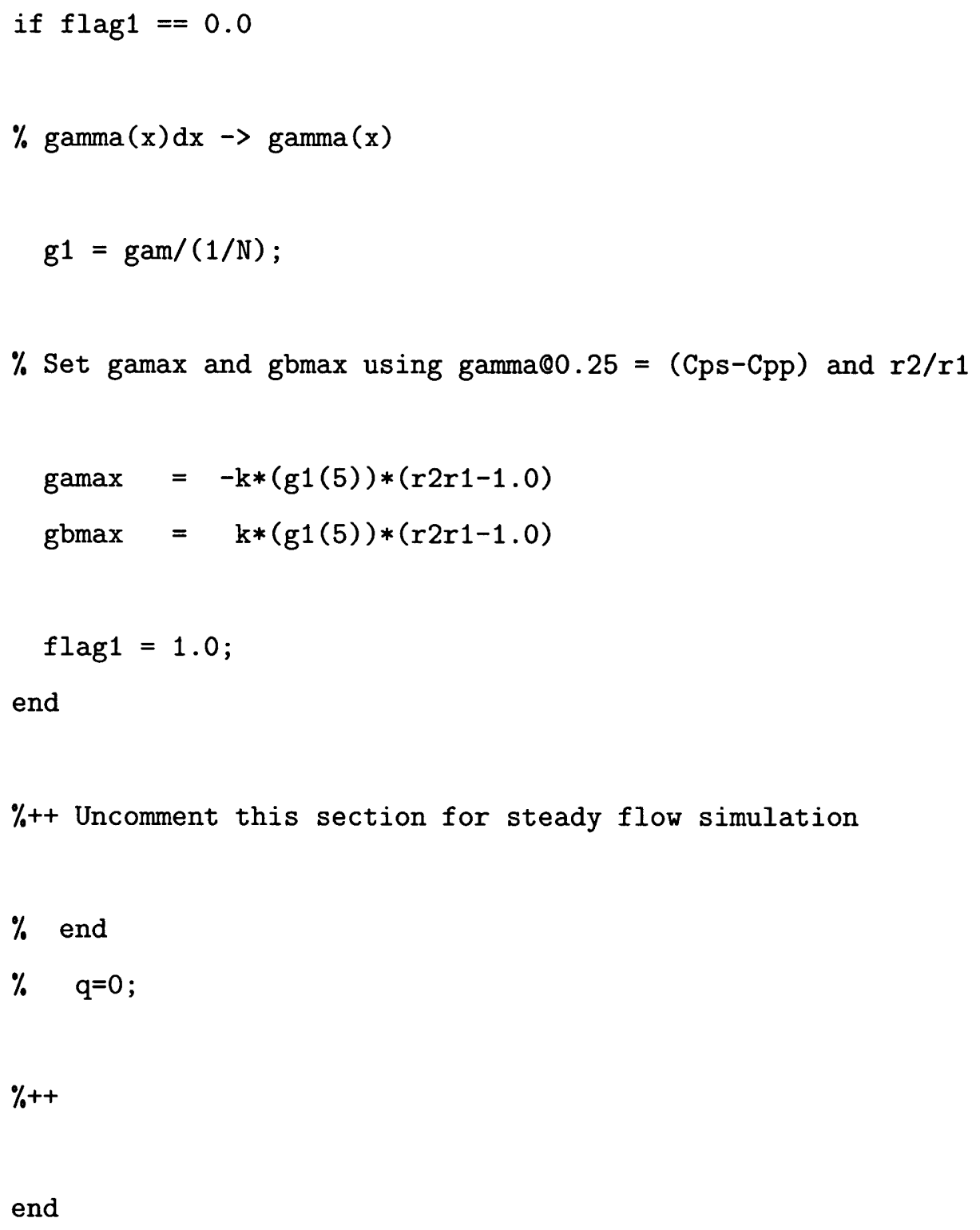


Filename: kernel.m

$\%$ Kernel function to be used with cascade model.

$\%$

$\%$ Written by Sanith Wijesing

\% MIT Gas Turbine Lab. July 1998.

\%---------

function $[t]=\operatorname{kernel}(e)$

global $\times 1$ sig beta1

$\mathrm{nu}=\operatorname{size}(e, 2)$

$\mathrm{sx}=\mathrm{x} 1 *$ ones $(1, \mathrm{nu})$;

zt1 $=s x-e ;$

$t=(-i * \exp (i * \operatorname{beta} 1) * \operatorname{coth}(p i *(z t 1) *(1 / \operatorname{sig}) * \exp (i *$ beta1 $)))$

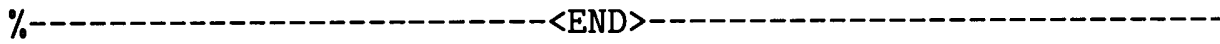

